Universidad Politécnica de Madrid

Facultad de Ciencias de la Actividad Física y del Deporte

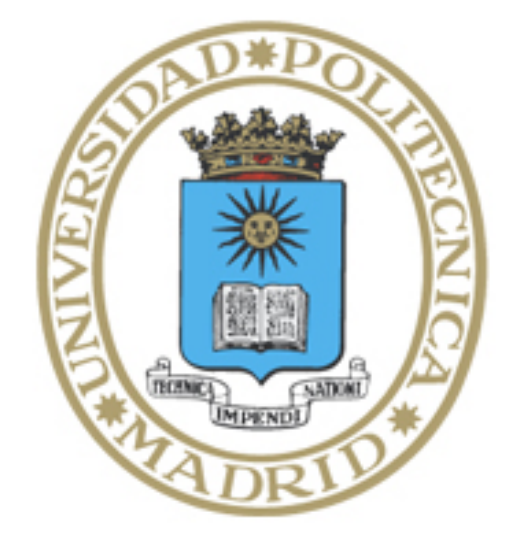

\title{
ANÁLISIS DE LA MARCHA: \\ EVALUACIÓN DE UN EXOESQUELETO APLICADO A \\ LA MARCHA ASISTIDA
}

Tesis Doctoral

María Gómez Jiménez

Licenciada en Ciencias de la Actividad Física y del Deporte

2016 

Departamento de Salud y Rendimiento Humano

Facultad de Ciencias de la Actividad Física y del Deporte

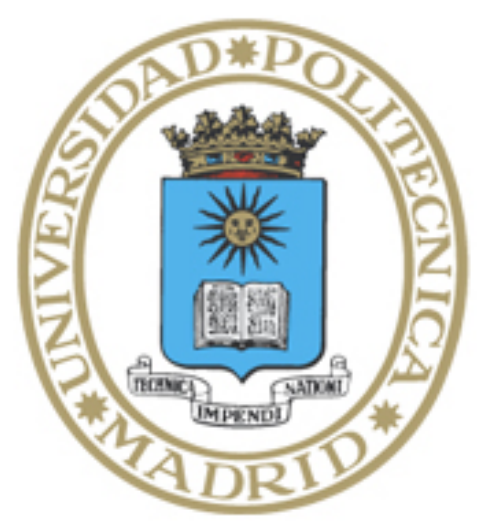

\title{
ANÁLISIS DE LA MARCHA: \\ EVALUACIÓN DE UN EXOESQUELETO APLICADO \\ A LA MARCHA ASISTIDA
}

\author{
María Gómez Jiménez \\ Licenciada en Ciencias de la Actividad Física y del Deporte
}

Directores:

Cristina López de Subijana Hernández

Doctora en Ciencias de la Actividad Física y del Deporte

Santiago Veiga Fernández

Doctor en Rendimiento Deportivo 



\section{TRIBUNAL DE LA TESIS}

Tribunal nombrado por el Mgfico. y Excmo. Sr. Rector de la Universidad Politécnica de Madrid el día,

PRESIDENTE D./Dña.

VOCAL D./Dña.

VOCAL D./Dña.

VOCAL D./Dña.

SECRETARIO/A D./Dña.

Realizado el acto de defensa y lectura de Tesis el día, en

CALIFICACIÓN

EL PRESIDENTE

LOS VOCALES

EL SECRETARIO 

"All truly great thoughts are conceived while walking"

Friedrich Nietzsche 



\section{Agradecimientos}

Muchas personas, cuando llega el momento de escribir los agradecimientos, describen lo duro del proceso vivido y las dificultades encontradas durante los últimos cuatro años. Es cierto que han existido momentos difíciles y se llega al final muy cansada, pero tengo que agradecer haber aprendido y disfrutado sobre todo.

Me gustaría agradecer, en primer lugar, a la Facultad de Ciencias de la Actividad Física y el Deporte, al INEF, a todos sus profesores, personal y alumnos que me han dado la fuerza para querer continuar dando clase en la que siento mi casa, y ser una motivación extra para realizar esta tesis doctoral.

Especial agradecimiento a mis directores de tesis, Cristina López de Subijana y Santiago Veiga, por toda su paciencia, dedicación y apoyo. A Cristina, agradecer también haber sido sobre todo mi mentora y amiga durante este tiempo.

Gracias al Departamento de Salud y Rendimiento Humano, por confiar en mí como docente e investigadora, y apostar por mi formación y mi proyección profesional.

Debo agradecer al Laboratorio de Biomecánica Deportiva, y a Enrique Navarro, por abrirme las puertas del laboratorio en 2010.

Gracias a Raquel, por su ayuda en el laboratorio y recogida de datos, y por estar y dar siempre, en lo profesional y lo personal.

Agradecer también a Maribel Barriopedro por las horas extra de estadística, y a los colaboradores del Laboratorio de Biomecánica que participaron en este proyecto.

A todos los voluntarios que ofrecieron su tiempo. Gracias.

Gracias a mis padres, por todo. Por todas las oportunidades que me han dado, por hacerme fuerte y empujarme siempre a ir hacia delante. Gracias a Pablo, por su tiempo, apoyo y calma.

Gracias a todos. 



\section{Resumen}

La marcha humana es el mecanismo de locomoción por el cual el cuerpo humano se traslada en línea recta gracias a una serie de movimientos coordinados de la pelvis y de las articulaciones del miembro inferior. Frecuentemente se encuentra influenciada por factores biomecánicos, anatómicos o patologías del sistema neuromusculoesquelético que modifican la forma de caminar de cada individuo. La lesión de médula espinal es una de las patologías que afectan el desarrollo normal de los patrones de la marcha por alteración de la movilidad, de la sensibilidad o del sistema nervioso autónomo. Aunque la lesión medular afecta otras funciones, además de la pérdida de función motora y sensorial, la recuperación de la capacidad de caminar es la mayor prioridad identificada por los pacientes durante la rehabilitación. Por ello, el desarrollo de dispositivos que faciliten la rehabilitación o compensación de la marcha es uno de los principales objetivos de diferentes grupos de investigación y empresas. En el contexto del proyecto Hybrid Technological Platform for Rehabilitation, Functional Compensation and Training of Gait in Spinal Cord Injury Patients se ha desarrollado un dispositivo que combina una órtesis activa (exoesqueleto) y un andador motorizado. Este sistema, como otros dispositivos, tiene el movimiento humano como estándar de referencia, no obstante no se evalúa de manera habitual, cómo es el patrón de la marcha reproducido y su similitud o diferencias con la marcha humana, o las modificaciones o adaptaciones en la interacción con el cuerpo del paciente. El presente estudio trata de examinar las características de la marcha normal en diversos grupos de población, y las diferencias con el patrón de marcha lenta. Finalmente, se pretende evaluar qué modificaciones y adaptaciones sufre el patrón de marcha lenta teórico al ser reproducido por el exoesqueleto. La presente investigación consiste en un estudio cuantitativo transversal desarrollado en dos etapas: estudio 1 y estudio 2 . En el estudio 1 se analizó el patrón de la marcha a velocidad libremente seleccionada (normal) y el patrón de la marcha a velocidad lenta $(0.25 \mathrm{~m} / \mathrm{s}$ ) en 62 sujetos distribuidos en grupos considerando el sexo y los percentiles 25,50 y 75 de estatura de la población española. Durante el estudio 2 se analizó el patrón de la marcha lenta reproducido por el dispositivo Hybrid a diferentes porcentajes de peso corporal (30\%, 50\% y 70\%) en diez sujetos seleccionados aleatoriamente de la muestra del estudio 1. En ambos estudios se obtuvieron variables espacio-temporales y cinemáticas mediante un sistema de captura de movimiento con 6 cámaras distribuidas a lo largo de un pasillo de marcha. Se calcularon las medias, las desviaciones estándar y el 95\% de intervalo de confianza, y el nivel alfa de significación se estableció en $\alpha=0.05$ para todas las pruebas estadísticas. 
Las principales diferencias en el patrón normal de la marcha se encontraron en los parámetros cinemáticos de hombres y mujeres, aunque también se presentaron diferencias entre los grupos en función de la estatura. Las mujeres mostraron mayor flexión de cadera y rodilla, y mayor extensión de tobillo que los hombres durante el ciclo normal, aunque la basculación lateral de la pelvis, mayor en las mujeres, y el desplazamiento lateral del centro de gravedad, mayor en los hombres, fueron los parámetros identificados como principales discriminantes entre sexos. La disminución de la velocidad de la marcha mostró similares adaptaciones y modificaciones en hombres y en mujeres, presentándose un aumento de la fase de apoyo y una disminución de la fase de oscilación, un retraso de los máximos y mínimos de flexoextensión de cadera, rodilla y tobillo, y una disminución del rango articular en las tres articulaciones. Asimismo, la basculación lateral de la pelvis y el movimiento vertical del centro de gravedad disminuyeron, mientras que el movimiento lateral del centro de gravedad y el ancho de paso aumentaron. Durante la evaluación del patrón de la marcha reproducido por el exoesqueleto se observó que las tres articulaciones del miembro inferior disminuían el rango de movimiento por la falta de fuerza de los motores para contrarrestar el peso corporal, incluso con un $70 \%$ de descarga de peso. Además, la transferencia de peso se encontró limitada por la falta de movimiento de la pelvis en el plano frontal y se sustituyó por un aumento de la inclinación del tronco y, por tanto, del movimiento lateral del centro de gravedad. Este hecho, junto al aumento del desplazamiento vertical del centro de gravedad, hizo del patrón de la marcha reproducido por el exoesqueleto un movimiento poco eficiente. En conclusión, se establecen patrones de marcha normal diferenciados por sexos, siendo la basculación lateral de la pelvis y el movimiento lateral del centro de gravedad los parámetros discriminantes más característicos entre sexos. Comparando la marcha a velocidad libremente seleccionada y la velocidad lenta, se concluye que ambos sexos utilizan estrategias similares para adaptar el patrón de la marcha a una velocidad lenta y se mantienen las características diferenciadoras entre hombres y mujeres. En relación a la evaluación del dispositivo Hybrid, se deduce que la falta de movimiento lateral de la pelvis condiciona la transferencia de peso y el aumento del rango de movimiento del centro de gravedad $y$, en consecuencia, tiene como resultado un patrón de la marcha poco eficiente. Este patrón no resultaría indicado para los procesos de rehabilitación o recuperación de la marcha, aunque podría considerarse adecuado para la compensación funcional de la bipedestación y la locomoción. 


\section{Abstract}

The human walking is a means of moving body forward using a repetitious and coordinated sequence of pelvis and lower limb motions. It is frequently influenced by biomechanical and anatomical factors or by musculoskeletal pathologies which modify the way of walking. The spinal injury is one of those pathologies which affect the normal pattern of walking, due to the alteration of the mobility, the sensory or the autonomic nervous system. Although the spinal injury affects many other body functions, apart from the motor and sensory ones, the main priority for patients is to recover the ability of walking. Consequently, the main objective of many research groups and private companies is the development of rehabilitation and compensation devices for walking. In this context, the Hybrid Technological Platform for Rehabilitation, Functional Compensation and Training of Gait in Spinal Cord Injury Patients project has developed a device which integrates an exoskeleton and a motorized smart walker. This system, as other similar devices, has the human movement as standard reference. Nevertheless, these devices are not usually evaluated on the way they reproduce the normal human pattern or on the modifications and in the interactions with the patient's body. The aim of the present study is to examine the normal walking characteristics, to analyze the differences between self-selected and low speed walking patterns, and to evaluate the modifications and adaptations of walking pattern when it is reproduced by the exoskeleton.

The present research is a quantitative cross-sectional study carried out in two phases: study 1 and study 2 . During the study 1 , the self-selected and the low speed $(0.25 \mathrm{~m} / \mathrm{s})$ walking patterns were analyzed in sixty-two people distributed in groups, according to sex and $25^{\text {th }}$, $50^{\text {th }}$ and $75^{\text {th }}$ percentiles of height for Spanish population. The study 2 analyzed the low speed walking pattern reproduced by the Hybrid system in three conditions: $30 \%, 50 \%$ and $70 \%$ of body weight support. To do this, ten subjects were randomly selected and analyzed from the people of study 1 . An optoelectronic system with six cameras was used to obtain spatial, temporal and kinematic parameters in both studies. Means, standard deviations and $95 \%$ confidence intervals of the study were calculated. The alpha level of significance was set at $\alpha=0.05$ for all statistical tests.

The main differences in normal gait pattern were found in kinematic parameters between men and women. The hip and the knee were more flexed and the ankle plantar flexion was higher in women than in men during normal gait cycle. Although the greater pelvic obliquity of women and the higher lateral movement of center of gravity of men were the most relevant discriminators between male and female gait patterns. Comparing self-selected and low speed walking patterns, both sexes showed similar adaptations and modifications. At low speed 
walking, men and women increased the stance phase ratio and decreased the swing phase ratio. The maximum and minimum peak flexion of hip, knee and ankle appeared after and the range of motion of them decreased during low speed walking. Furthermore, the pelvic obliquity and the vertical movement of the center of gravity decreased, whereas the lateral movement of center of gravity and step width increased. Evaluating the gait pattern reproduced by the exoskeleton, a decrease of lower limb range of motion was observed. This was probably due to the lack of strength of the engines, which were not able to control the body weight, even with the $70 \%$ supported. Moreover, the weight transfer from one limb to the contralateral side was restricted due to the lack of pelvis obliquity. This movement deficiency was replaced by the lateral torso sway and, consequently, the increase of lateral movement of the center of gravity. This fact, as well as the increase of the vertical displacement of the center of gravity, made inefficient the gait pattern reproduced by the exoskeleton.

In conclusion, different gait patterns of both sexes have been determined, being pelvis obliquity and lateral movement of center of gravity the most relevant discriminators between male and female gait patterns. Comparing self-selected and low speed walking patterns, it was concluded that both sexes use similar strategies for adapting the gait pattern to a low speed, and therefore, the differentiating characteristics of normal gait are maintained. Regarding the Hybrid system evaluation, it was determined that the gait pattern reproduced by the exoskeleton is inefficient. This was due to the lack of pelvis obliquity and the increase of the center of gravity displacement. Consequently, whereas the walking pattern reproduced by the exoskeleton would not be appropriated for the rehabilitation process, it could be considered suitable for functional compensation of walking and standing. 
1. Introducción 1

1.1. Marcha humana 1

1.1.1. Descripción de la Marcha humana 1

1.1.1.1. Fases y Eventos de la Marcha humana 1

1.1.1.2. Parámetros Espacio-temporales 8

1.1.1.3. Parámetros Cinemáticos 9

1.1.2. Alteraciones de la Marcha en Patologías 14

1.1.3. Otros Factores que Modifican el Patrón de la Marcha 15

1.1.3.1. Influencia del sexo y estatura en el patrón de la marcha $\quad 16$

1.1.3.2. Influencia de la velocidad en el patrón de la marcha 17

1.1.4. Instrumentos de Valoración de la Marcha 19

1.1.5. Aplicaciones del Análisis de la Marcha 23

1.2. Lesión Medular 25

1.2.1. Causas de Lesión Medular: Epidemiología 25

1.2.2. Tipos de Lesión Medular y Clasificación 26

1.2.3. Consecuencias e Implicaciones de la Lesión Medular 29

1.2.4. Necesidades de Lesionados Medulares: Importancia de la 31 Bipedestación

1.3. Robots 33

1.3.1. Dispositivos para la Rehabilitación y Compensación de la Marcha 33

1.3.1.1. Exoesqueletos combinados con tapiz rodante 34

1.3.1.2. Exoesqueletos con desplazamiento sobre el suelo 35

1.3.2. Descripción del sistema HYBRID y su Funcionamiento 38

1.3.3. Rehabilitación con Exoesqueletos en pacientes con Lesión Medular $\quad 40$

\section{Planteamiento del problema, Objetivos e Hipótesis 43}

2.1. Planteamiento del problema 45

2.2. Objetivos de la Investigación 46

2.3. Hipótesis de la Investigación 47

3. Metodología 49

3.1. Diseño de Investigación 51

3.2. Muestra 52 
3.2.2. Muestra Sujetos Sanos para prueba con dispositivo Hybrid

3.4.1. Estudio 1: Patrón de la Marcha a Velocidad Normal y Lenta

3.4.1.1. Protocolo

3.4.1.2. Modelo de marcadores y Modelo mecánico

3.4.2. Estudio 2: Análisis de la marcha con dispositivo Hybrid

3.4.2.1. Protocolo

3.4.2.2. Modelo de marcadores y Modelo mecánico

3.5.1. Medidas del sujeto

3.5.4. Exportado de trayectorias

3.7.2. Comparación patrón velocidad normal y patrón velocidad lenta

3.7.3. Comparación Patrón porcentajes de descarga de dispositivo Hybrid con el patrón velocidad lenta

\section{Resultados}

4.1. Análisis de las diferencias en la Marcha Normal en función del sexo y de la 73 estatura de los sujetos

4.1.1. Parámetros espacio-temporales

4.1.1.1. Velocidad y cadencia

4.1.1.2. Distancias 75

4.1.1.3. Tiempos 77

4.1.2. Parámetros cinemáticos 80

4.1.2.1. Pelvis y centro de gravedad 80

4.1.2.2. Cadera 
4.2. Comparativa de los patrones de Marcha a velocidad Lenta y los patrones de la 88 Marcha a velocidad Normal

4.2.1. Parámetros espacio-temporales 88

4.2.1.1. Velocidad y cadencia 88

4.2.1.2. Distancias 90

4.2.1.3. Tiempos 93

4.2.2. Parámetros cinemáticos $\quad 97$

4.2.2.1. Pelvis y centro de gravedad 97

4.2.2.2. Cadera 100

4.2.2.3. Rodilla 103

4.2.2.4. Tobillo 106

4.3. Comparativa de los patrones de la marcha reproducidos por el Exoesqueleto con el 110 $30 \%, 50 \%$ y $70 \%$ de descarga de peso corporal y los patrones de la Marcha a velocidad Lenta

4.3.1. Parámetros espacio-temporales $\quad 110$

4.3.1.1. Velocidad y cadencia 110

4.3.1.2. Distancias 112

4.3.1.3. Tiempos 114

4.3.2. Parámetros cinemáticos 116

4.3.2.1. Centro de gravedad 116

4.3.2.2. Cadera 118

4.3.2.3. Rodilla 120

$\begin{array}{ll}\text { 4.3.2.4. Tobillo } & 122\end{array}$

$\begin{array}{ll}\text { 5. Discusión } & 125\end{array}$

5.1. Análisis de las diferencias en la Marcha Normal en función del sexo y de la 127 estatura de los sujetos

5.2. Comparativa de los patrones de Marcha a velocidad Lenta y los patrones de la 133 Marcha a velocidad Normal

5.3. Comparativa de los patrones de la marcha reproducidos por el Exoesqueleto con el 140 $30 \%, 50 \%$ y $70 \%$ de descarga de peso corporal y los patrones de la Marcha a velocidad Lenta

6. Conclusiones 
ÍNDICE DE CONTENIDOS

7. Aportaciones Finales

7.1. Aplicaciones del Estudio

7.2. Limitaciones del Estudio

7.3. Futuras Líneas de Investigación

Bibliografía

Anexos

Anexo I. Consentimiento Informado

Anexo II. Comité de Ética de la Universidad Politécnica de Madrid 
Tabla 1. Cronograma de las tareas del proyecto realizadas durante la investigación

Tabla 2. Características antropométricas de la muestra

Tabla 3. Relación de Variables Espaciotemporales estudiadas por otros autores y en el presente estudio

Tabla 4. Relación de Variables Cinemáticas estudiadas por otros autores y en el presente estudio

Tabla 5. Parámetros Espacio-temporales: Velocidad y Cadencia (Grupos por estatura)

Tabla 6. Parámetros Espacio-temporales: Velocidad y Cadencia (Hombres y Mujeres)

Tabla 7. Parámetros Espacio-temporales: Distancias (Grupos por estatura)

Tabla 8. Parámetros Espacio-temporales: Distancias (Hombres y Mujeres)

Tabla 9. Parámetros Espacio-temporales: Tiempos (Grupos por estatura)

Tabla 10. Parámetros Espacio-temporales: Tiempos (Hombres y mujeres)

Tabla 11. Parámetros Cinemáticos: Pelvis y Centro de Gravedad (Grupos por estatura)

Tabla 12. Parámetros Cinemáticos: Pelvis y Centro de Gravedad (Hombres y mujeres)

Tabla 13. Parámetros Cinemáticos: Cadera (Grupos por estatura)

Tabla 14. Parámetros Cinemáticos: Cadera (Hombres y Mujeres)

Tabla 15. Parámetros Cinemáticos: Rodilla (Grupos por estatura)

Tabla 16. Parámetros Cinemáticos: Rodilla (Hombres y mujeres)

Tabla 17. Parámetros Cinemáticos: Tobillo (Grupo por estatura)

Tabla 18. Parámetros Cinemáticos: Tobillo (Hombres y Mujeres)

Tabla 19. Parámetros Espacio-temporales: Velocidad y Cadencia (Velocidad Normal y Velocidad Lenta)

Tabla 20. Parámetros Espacio-temporales: Distancias (Velocidad Normal y Velocidad Lenta)

Tabla 21. Parámetros Espacio-temporales: Tiempos (Velocidad Normal y Velocidad Lenta)

Tabla 22. Parámetros Cinemáticos: Pelvis y Centro de Gravedad (Velocidad Normal y Velocidad Lenta)

Tabla 23. Parámetros Cinemáticos: Cadera (Velocidad Normal y Velocidad Lenta)

Tabla 24. Parámetros Cinemáticos: Rodilla (Velocidad Normal y Velocidad Lenta)

Tabla 25. Parámetros Cinemáticos: Tobillo (Velocidad Normal y Velocidad Lenta)

Tabla 26. Parámetros Espacio-temporales: Velocidad y Cadencia (Velocidad Lenta y Porcentajes de Descarga con Exoesqueleto)

Tabla 27. Parámetros Espacio-temporales: Distancias (Velocidad Lenta y Porcentajes de Descarga con Exoesqueleto)

Tabla 28. Parámetros Espacio-temporales: Tiempos (Velocidad Lenta y Porcentajes de Descarga con Exoesqueleto)

Tabla 29.Parámetros Cinemáticos: Centro de Gravedad (Velocidad Lenta y Porcentajes de Descarga con Exoesqueleto)

Tabla 30. Parámetros Cinemáticos: Cadera (Velocidad Lenta y Porcentajes de Descarga con Exoesqueleto) 
ÍNDICE DE TABLAS

Tabla 31. Parámetros Cinemáticos: Rodilla (Velocidad Lenta y Porcentajes de Descarga con

Exoesqueleto)

Tabla 32. Parámetros Cinemáticos: Tobillo (Velocidad Lenta y Porcentajes de Descarga con Exoesqueleto) 
Figura 1. Divisiones del ciclo de la marcha $\quad 5$

Figura 2. Fases de apoyo doble y apoyo simple en el ciclo de la marcha 6

Figura 3. Fases del Ciclo de la Marcha $\quad 7$

Figura 4. Esquema de los parámetros espaciales del ciclo de la marcha $\quad 8$

Figura 5. Desplazamiento vertical y medio-lateral del centro de gravedad durante el ciclo de la 10 marcha

Figura 6. Movimientos de la pelvis durante el ciclo de la marcha

Figura 7. Movimiento de flexo-extensión de la cadera durante el ciclo de la marcha (plano sagital) 12

Figura 8. Movimiento de flexo-extensión de la rodilla durante el ciclo de la marcha (plano sagital) 13

Figura 9. Movimiento de flexo-extensión del tobillo durante el ciclo de la marcha (plano sagital)

Figura 10. Estudio de la Marcha por Eadweard Muybridge

Figura 11. Sistema de marcadores para la calibración: kit de calibración de VICON. En verde “Calibration Wand" para la calibración dinámica y en rojo "L-Frame" para la calibración estática

Figura 12. Modelo para la colocación de marcadores modificado de Helen Hayes Model

Figura 13. Cámara de sistema VICON. De izquierda a derecha: Esquema de la disposición de LEDs alrededor de la lente y Fotografía de una cámara

Figura 14. Marcadores reflectantes esféricos

Figura 15. Causas de lesión medular no traumática

Figura 16. Escala de Medición de la Discapacidad de la Asociación Americana de Lesión Medular

Figura 17. Grado de afectación de la lesión medular según la escala ASIA

Figura 18. Organización longitudinal de la médula espinal y representación de sus funciones principales

Figura 19. Exoesqueletos para el incremento de fuerza en sujetos sanos. De izquierda a derecha: BLEEX, MIT Exoskeleton, HAL-5 y Nurse-Assisting Exoskeleton

Figura 20. Exoesqueletos combinados con tapiz rodante: (a) Lokomat, (b) ReoAmbulator, (c) ALEX y (d) LOPES

Figura 21. Exoesqueletos internacionales con desplazamiento sobre el suelo. De izquierda a 36 derecha: ReWalk, Ekso Bionics y Rex Bionics

Figura 22. Exoesqueletos nacionales con desplazamiento sobre el suelo. De izquierda a derecha: H2 Exoesqueleto (Technaid) y Atlas 2020 (Marsi Bionics)

Figura 23. Exosqueletos con desplazamiento sobre suelo y sistema de descarga de peso. De izquierda a derecha: WalkTrainer y NaTUre-gait

Figura 24. Dispositivo HYBRID. De izquierda a derecha: Andador motorizado con sistema de descarga de peso y órtesis activa con control de cadera, rodilla y tobillo

Figura 25. Estructura REMOVI

Figura 26. Usuaria del dispositivo HYBRID en el Hospital de Parapléjicos de Toledo 
Figura 27. Distribución de la muestra en grupos de estatura $\quad 53$

Figura 28. Esquema de espacio de captura $\quad 54$

Figura 29. Protocolo de medida del Estudio $1 \quad 56$

Figura 30. Modelo Plug in Gait para la colocación de marcadores en miembro inferior 57

$\begin{array}{ll}\text { Figura 31. Modelo mecánico Plug in Gait } & 58\end{array}$

Figura 32. Protocolo de medida del Estudio 2

Figura 33. Modelo de marcadores estático y dinámico adaptado al exoesqueleto 60

Figura 34. Workstation. Subject Measurements 61

Figura 35. Workstation. Identificación de eventos en ciclo de la marcha 62

Figura 36. Workstation. Woltring Filtering Routine 63

Figura 37. Workstation. Run Static Gait Model 63

Figura 38. Workstation. Run Dynamic Gait Model 64

Figura 39. Bodybuilder. Exportado de trayectorias de marcadores y centros articulares 64

Figura 40. Análisis estadístico para establecer los patrones normales de la marcha 68

Figura 41. Ancho de paso en mujeres (Grupos de estatura) 93

Figura 42. Porcentaje Apoyo en hombres (Velocidad Normal y Velocidad Lenta) 94

Figura 43. Porcentaje Oscilación en hombres (Velocidad Normal y Velocidad Lenta) 94

Figura 44. Centro de Gravedad (plano sagital) en hombres (Grupos de estatura) 97

Figura 45. Centro de gravedad (plano frontal) en mujeres (Velocidad Normal y Velocidad Lenta) 98

Figura 46. Flexión Máxima de Rodilla (\%) en hombres (Velocidad Normal y Velocidad Lenta) 105

Figura 47. Flexión Máxima de Rodilla (\%) en mujeres (Velocidad Normal y Velocidad Lenta) 106

Figura 48. Extensión Máxima de Tobillo (\%) en hombres (Velocidad Normal y Velocidad Lenta) 107

Figura 49. Cinemática de la cadera. Diferencias entre el patrón de hombres y mujeres 128

Figura 50. Cinemática de la rodilla. Diferencias entre el patrón de hombres y mujeres 129

Figura 51. Cinemática del tobillo. Diferencias entre el patrón de hombres y mujeres 130

Figura 52. Cinemática del tobillo. Diferencias entre los grupos de estatura de los hombres 131

Figura 53. Cinemática de la cadera. Diferencias entre marcha normal y marcha lenta en hombres 134

Figura 54. Cinemática de la cadera. Diferencias entre marcha normal y marcha lenta en mujeres 135

Figura 55. Cinemática de la rodilla. Diferencias entre marcha normal y marcha lenta en hombres 136

Figura 56. Cinemática de la rodilla. Diferencias entre marcha normal y marcha lenta en mujeres 136

Figura 57. Cinemática del tobillo. Diferencias entre marcha normal y marcha lenta en hombres 137

Figura 58. Cinemática del tobillo. Diferencias entre marcha normal y marcha lenta en mujeres 138

Figura 59. Diferencias en la distribución temporal entre los porcentajes de descarga y la marcha 141 lenta (30\%, 50\% y $70 \%)$ (Hombres y Mujeres)

Figura 60. Cinemática de la cadera durante el instante de extensión máxima 142

Figura 61. Cinemática de la rodilla durante el instante de extensión máxima 143

Figura 62. Cinemática del tobillo durante el instante de extensión máxima 144 
CG Centro de gravedad

DPC Descarga de peso corporal

EIAS Espinas ilíacas anterosuperiores 

1. Introducción 



\subsection{Marcha humana}

\subsubsection{Descripción de la Marcha humana}

La marcha humana es el mecanismo de locomoción por el cual el cuerpo humano se traslada en línea recta gracias a una serie de movimientos coordinados de las grandes articulaciones de los miembros inferiores. Este movimiento requiere de la combinación del sistema neurológico y musculoesquelético para convertir los movimientos verticales de las extremidades inferiores en un suave movimiento de traslación y avance de la cabeza y el tronco, lo que permite mantener la línea de los ojos relativamente estable durante el proceso.

Andar implica un patrón de movimiento repetitivo y cíclico en cada extremidad inferior para que cada pie se traslade de una posición de apoyo a la siguiente. Este carácter cíclico de la marcha permite identificar la unidad de este movimiento, el ciclo, para describir el proceso de una forma precisa. Por lo tanto, cuando se analiza cualquier patrón de la marcha humana, el ciclo de la marcha se considera como la unidad fundamental delimitado entre el primer apoyo del talón de un pie y el segundo apoyo del mismo talón (Chambers, \& Sutherland, 2002; Perry, \& Burnfield, 2010; Plas, Viel, \& Blanc, 1984; Sánchez Lacuesta, 1999; Viel, \& Plas, 2002).

\subsubsection{Fases y Eventos de la Marcha humana}

El ciclo de la marcha está dividido en dos grandes fases, la fase de apoyo y la fase de oscilación (Figura 1). La fase de apoyo (stance) se define como el periodo de tiempo en el que el pie está en contacto con el suelo. Esta comienza con el instante de contacto inicial, que en la marcha normal implica el contacto del talón, y termina con el despegue del pie, cuando la fase de oscilación (swing) comienza. Esta fase, a su vez, terminará cuando el talón contacte de nuevo con el suelo (Perry, \& Burnfield, 2010; Viel, \& Plas, 2002).

Cuando el periodo de tiempo de un ciclo completo se normaliza $(100 \%)$, cada uno de los eventos que definen las diferentes fases suceden en un porcentaje específico del ciclo. El contacto inicial (initial contact) que define el comienzo y el final del ciclo sucederán en el $0 \%$ y el $100 \%$ del ciclo de la marcha. Durante la marcha normal, el despegue del pie y comienzo de la fase de oscilación, sucede aproximadamente al $60 \%$ del ciclo. Por lo tanto, la fase de apoyo representará el $60 \%$ del ciclo, mientras que la fase de oscilación correspondería al $40 \%$ (Perry, \& Burnfield, 2010; Viel, \& Plas, 2002).

Estudiando el ciclo de una extremidad inferior en particular (homolateral) y comparándolo con la extremidad contralateral (el lado opuesto), el despegue del pie sucede al $10 \%$ del ciclo contralateral, y el contacto del talón al $50 \%$ del ciclo de la extremidad contralateral. Esto significa que durante la marcha aparecen dos periodos de doble apoyo en los que ambos pies 


\section{INTRODUCCIÓN}

están en el suelo y que puede considerarse como una fase de reestabilización entre dos apoyos monopodales. Cada uno de estos periodos constituye un $10 \%$ del ciclo (Figura 2).

El primer doble apoyo sucede inmediatamente después del contacto inicial (del $0 \%$ a $10 \%$ del ciclo) y se corresponde con una fase de amortiguación y deceleración (loading response) en el que el impacto inicial es absorbido y el peso corporal se traslada de una extremidad inferior a la otra. Esto es seguido de una fase de apoyo monopodal que ocupa alrededor de un $40 \%$ del ciclo, en el que la pierna contralateral estará en fase de oscilación (Perry, \& Burnfield, 2010; Viel, \& Plas, 2002).

El periodo de apoyo monopodal se subdivide a su vez en fase media de apoyo (mid stance) y fase terminal de apoyo (terminal stance). La fase media de apoyo sucede del $10 \%$ al $30 \%$ del ciclo de la marcha y es el periodo en el que el cuerpo progresa sobre un apoyo estable. En la marcha normal, esta fase termina cuando el peso corporal cae perpendicular sobre la parte media del pie. A continuación, del 30\% al 50\% del ciclo de la marcha encontramos la fase final de apoyo, que comenzará con la elevación del talón y terminará cuando el talón contralateral contacta con el suelo. Durante esta fase el peso corporal progresará hacia la pierna de apoyo (Perry, \& Burnfield, 2010; Viel, \& Plas, 2002).

El segundo periodo de doble apoyo se conoce también como fase previa a la oscilación (pre swing). Éste comenzará alrededor del 50\% del ciclo de la marcha y durará hasta el despegue del pie que estaba en apoyo. El comienzo de esta fase sucederá en el mismo instante del contacto inicial del talón contralateral y coincidirá con la fase de amortiguación y deceleración de la extremidad contralateral. Durante esta fase, la extremidad progresa desde una posición general de extensión a una posición de flexión y, aunque sigue siendo un periodo de apoyo, es funcionalmente importante en la preparación de la extremidad para la fase de oscilación (Perry, \& Burnfield, 2010; Viel, \& Plas, 2002).

El despegue del pie marca el inicio de la fase de oscilación. Esta fase se subdivide a su vez en tres fases: fase inicial de oscilación, fase media de oscilación y fase terminal de oscilación. La fase inicial de oscilación (initial swing) sucede del $60 \%$ al $73 \%$ del ciclo de la marcha y es el periodo en el que la extremidad avanza y el pie se libera. Comienza en el despegue del pie y termina cuando la pierna en oscilación llega a la altura de la pierna que está en apoyo. 


\section{CICLO}

\section{(Gait Cycle)}

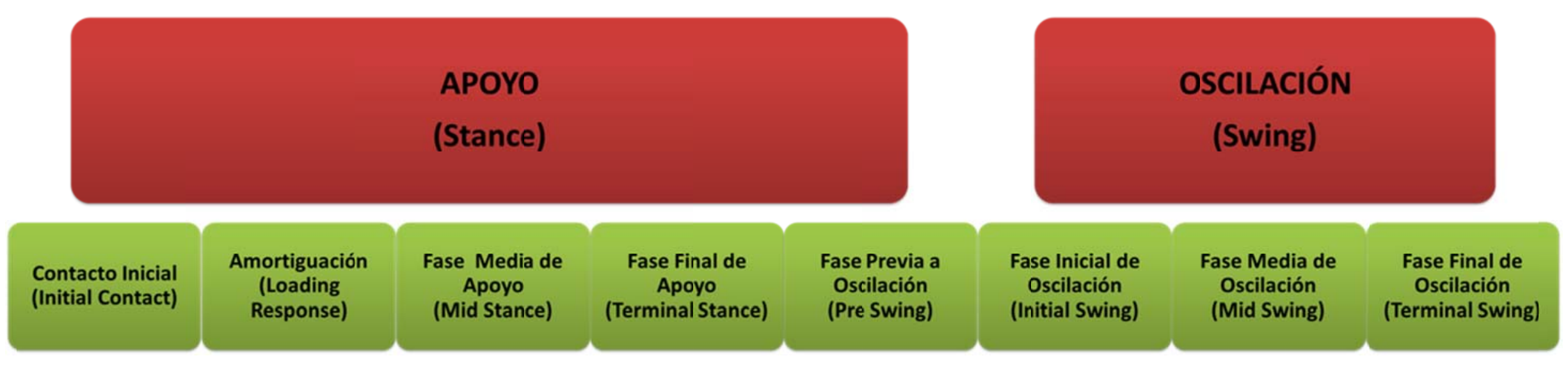

Figura 1. Divisiones del ciclo de la marcha (adaptado de Perry, 1992). 


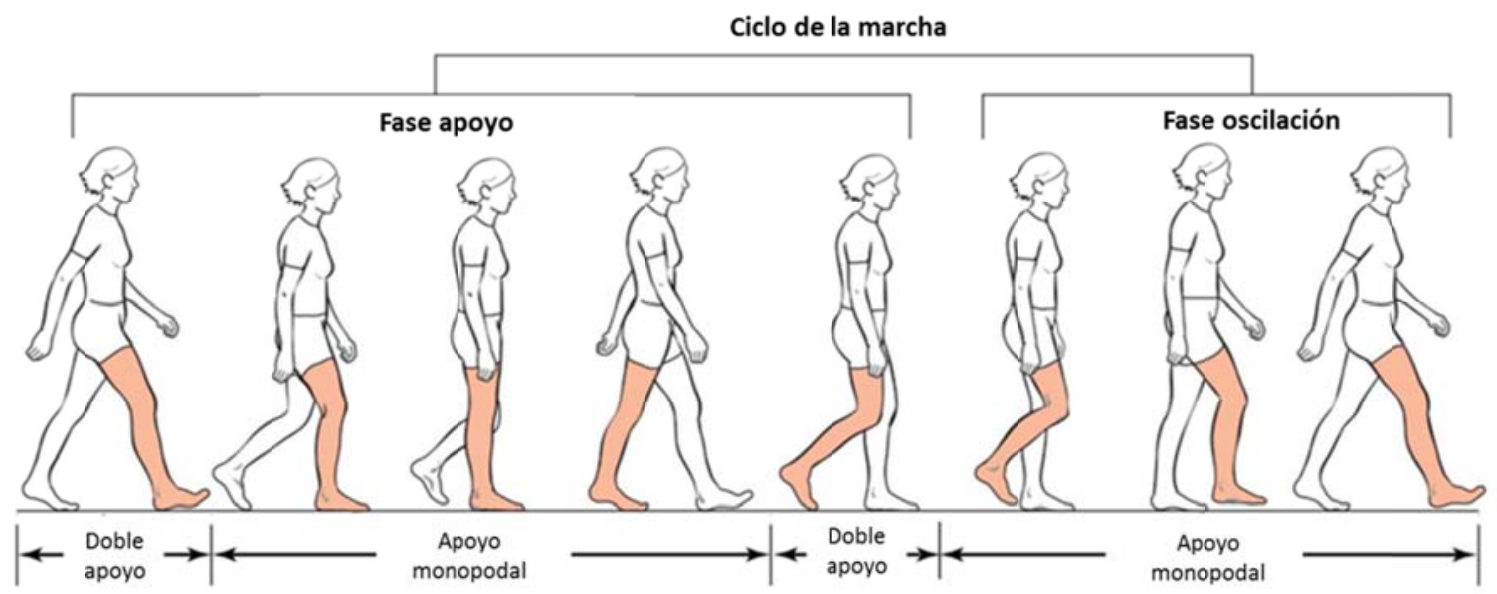

Figura 2. Fases de apoyo doble y apoyo simple en el ciclo de la marcha (adaptado de Studyblue, 2015).

A continuación, del $73 \%$ al $87 \%$ del ciclo, se encuentra la fase media de oscillación (mid swing) en la que la extremidad avanza hacia delante y la tibia se sitúa vertical y perpendicular al plano del suelo con el mismo objetivo funcional que en la fase anterior. La fase final de oscilación (terminal swing) es la fase final del ciclo de la marcha, en el que se produce una deceleración y la extremidad se prepara para el contacto del talón con el suelo (Figura 3) (Perry, \& Burnfield, 2010; Viel, \& Plas, 2002).

Es importante remarcar que aunque generalmente esta terminología se refiere a una extremidad, también se aplicaría a la extremidad contralateral que estará medio ciclo detrás o delante en una marcha humana normal. Por lo que, el primer doble apoyo en una extremidad corresponderá con el segundo doble apoyo de la extremidad contralateral (Perry, \& Burnfield, 2010; Viel, \& Plas, 2002). 


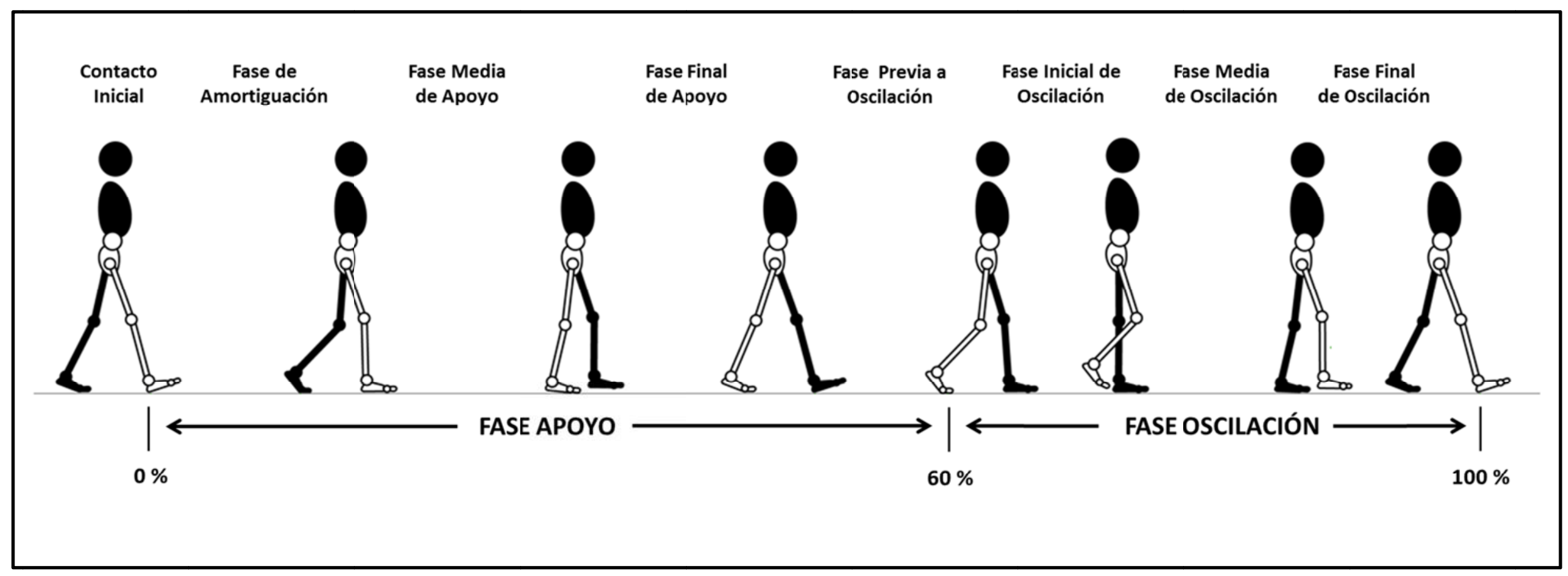

Figura 3. Fases del Ciclo de la Marcha (adaptado de Benchmarking Bipedal Locomotion, 2015). 


\subsubsection{Parámetros Espacio-temporales}

Otra forma de caracterizar, o definir la marcha humana, es a través de parámetros espaciales como la longitud de paso o la longitud de zancada, que, a su vez, se pueden combinar con parámetros temporales como la velocidad de la marcha o la cadencia. La longitud de paso se define como la distancia entre el punto de contacto con el suelo de uno de los pies y el mismo punto de contacto con el suelo del pie contario. Durante la marcha normal, se definiría la "longitud del paso derecho" como la distancia entre el punto de contacto dell talón izquierdo y el punto de contacto del talón derecho y, por lo tanto, la "longitud del paso izquierdo" será la distancia entre el punto de contacto del talón derecho y el punto de contacto del talón izquierdo. El tiempo de paso es el periodo de tiempo, expresado en segundos (s), medido desde el contacto de un pie al mismo evento del pie contrario. La longitud de zancada es la distancia recorrida durante un ciclo completo de la marcha y representa la suma de las longitudes de un paso derecho y un paso izquierdo, y se mide desde el punto de contacto de un pie con el suelo al siguiente punto de contacto con el suelo del mismo pie. En la marcha normal o no patológica, las longitudes de los pasos derecho e izquierdo deberían ser simétricas siempre y cuando la persona ande en línea recta.

Otro parámetro espacial que determina un patrón de marcha normal es la anchura del paso, que se define como la distancia entre los pies, y que normalmente se mide entre los centros articulares de cada tobillo. Este parámetro es fundamental a la hora de determinar problemas de equilibrio durante la marcha, ya que tiende a aumentar en condiciones de inestabilidad para aumentar la base de sustentación (Kirtley, 2006) (Figura 4).

\section{longitud zancada}

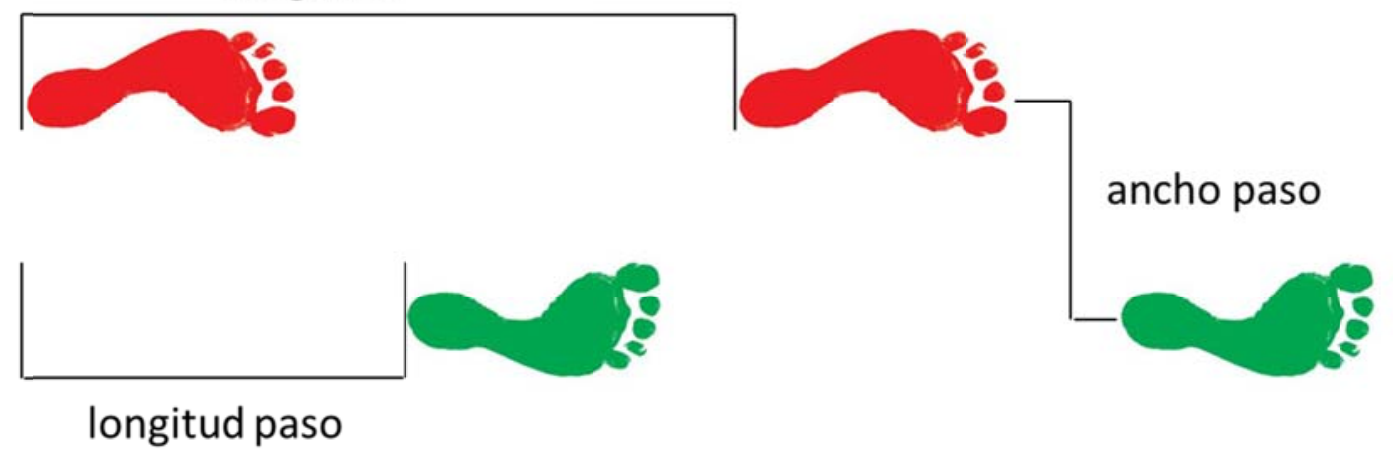

Figura 4. Esquema de los parámetros espaciales del ciclo de la marcha.

Los parámetros temporales que se manejan principalmente en la caracterización de un patrón de la marcha son: la cadencia y la velocidad de la marcha. La cadencia se define como la 
cantidad de pasos en un periodo de tiempo determinado, normalmente número de pasos por minuto. Por su parte, la velocidad de la marcha se expresa como la relación de la distancia recorrida por tiempo, tanto en centímetros por segundo $(\mathrm{cm} / \mathrm{s})$, metros por segundo $(\mathrm{m} / \mathrm{s})$ o como metros por minuto $(\mathrm{m} / \mathrm{min})$. La velocidad y la cadencia están íntimamente relacionadas con la longitud de zancada; un adulto y un niño son capaces de andar a la misma velocidad, pero puesto que el niño tiene una longitud de zancada menor, tendrá que tener una cadencia mayor para conseguir avanzar a la misma velocidad que un adulto. Cuando hablamos de velocidad normal de la marcha nos referimos a la velocidad seleccionada voluntariamente por cada individuo y que, generalmente, es la más eficiente, en términos de consumo energético, para esa persona (Chambers, \& Sutherland, 2002; Perry, \& Burnfield, 2010; Plas et al., 1984; Sánchez Lacuesta, 1999; Viel, \& Plas, 2002).

\subsubsection{Parámetros Cinemáticos}

Los parámetros cinemáticos estudiados miden el rango de movimiento dinámico de una articulación o segmento durante el ciclo de la marcha. Fundamentalmente se estudiarán el movimiento del centro de gravedad, pelvis, cadera, rodilla y tobillo.

La trayectoria descrita por el centro de gravedad durante el ciclo de la marcha se corresponde con una doble curva suave y ondulante en dirección cráneo-caudal (5 $\mathrm{cm}$ de amplitud) y medio-lateral ( $4 \mathrm{~cm}$ de amplitud). En el plano sagital, se describe un movimiento ascendente y descendente del centro de gravedad mostrando dos picos máximos de movimiento ascendente durante el $25 \%$ y $75 \%$ del ciclo correspondientes al final de la fase media de apoyo de la pierna que soporta el peso. Por tanto, en el 50\% del ciclo se encontrará el máximo de movimiento descendente. En el plano frontal, el movimiento lateral descrito durante el ciclo de la marcha corresponderá con el traslado del peso hacia la pierna de apoyo (Neumann, 2002; Perry, \& Burnfield, 2010; Saunders, Inman, \& Eberhart, 1953; Viel, \& Plas, 2002) (Figura $5)$. 


\section{Desplazamiento vertical del centro de gravedad}

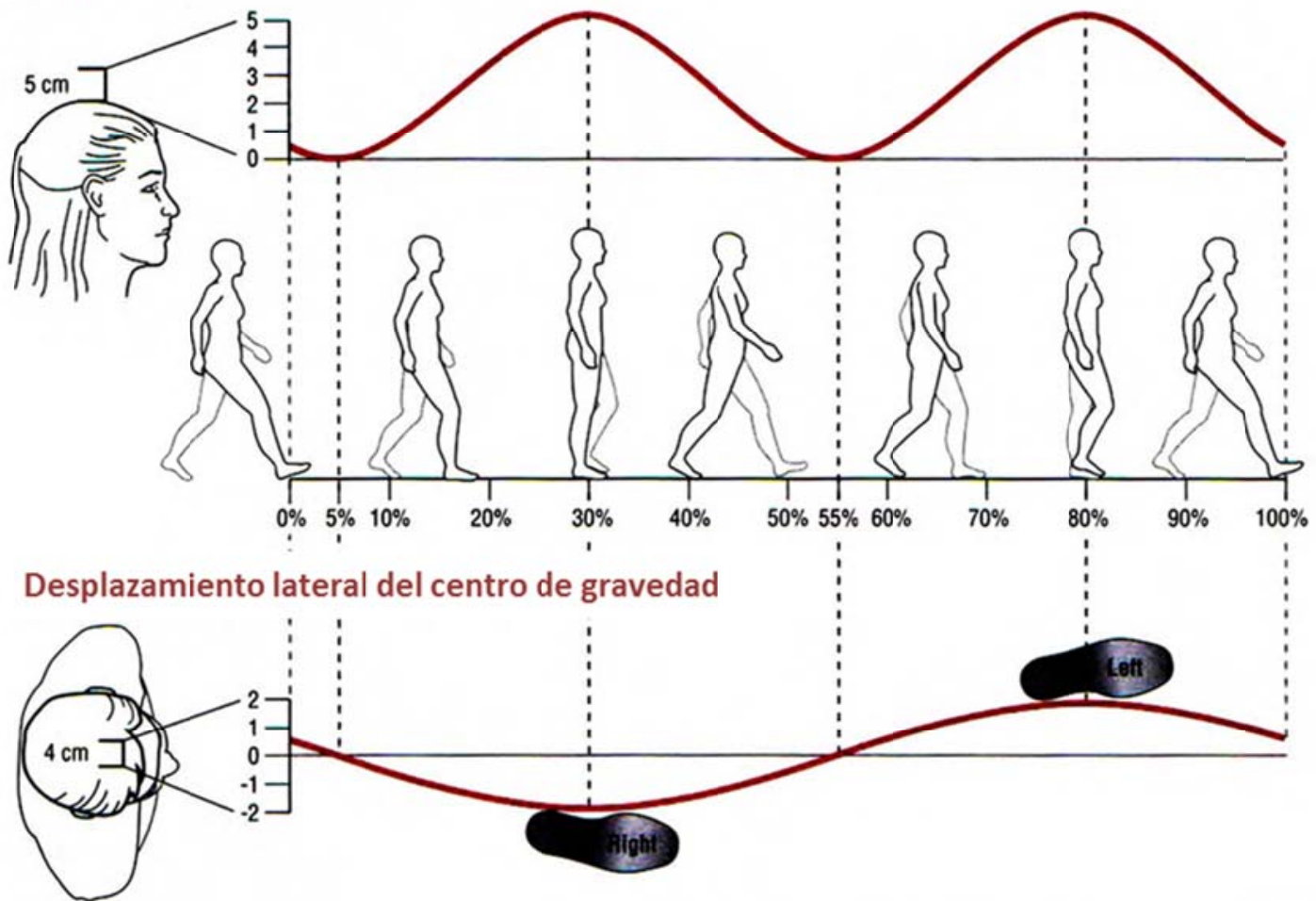

Figura 5. Desplazamiento vertical y medio-lateral del centro de gravedad durante el ciclo de la marcha (adaptado de Neumann, 2002).

A través de estas trayectorias sinusoidales del centro de gravedad se consigue trasladar el cuerpo en línea recta con el menor gasto de energía posible. Saunders et al. (1953) definieron cuales eran los seis determinantes básicos de la marcha para el mantenimiento de la trayectoria sinusoidal del centro de gravedad. La ausencia o deficiencia en uno de estos movimientos afectaría directamente a la suavidad de la trayectoria del centro de gravedad en los planos sagital y frontal. Estos seis determinantes son: 1) la rotación de la pelvis en el plano transversal; 2) la inclinación o caída lateral de la pelvis en el plano frontal; 3) la flexión de la rodilla en apoyo; los movimientos de 4) pie y 5) tobillo; y el 6) desplazamiento lateral de la pelvis relacionado con la aducción y abducción de la cadera. La pérdida o el compromiso de dos o más de estos determinantes, producirán compensaciones y una marcha poco eficiente. Aunque estos determinantes han sido defendidos y rebatidos por autores posteriores (Della Croce, Riley, Lelas, \& Kerrigan, 2001; Gard, \& Childress, 1999; Kerrigan, Della Croce, Marciello, \& Riley, 2000; Kerrigan, Riley, Lelas, \& Della Croce, 2001; Kuo, 2007), todos ellos, sostienen la importancia del movimiento de cadera, rodilla y tobillo para el mantenimiento del desplazamiento natural del centro de gravedad. También Perry (1992) describió cuatro prerrequisitos para un marcha normal y eficiente que implica un movimiento suave del centro 
de gravedad: 1) la estabilidad del pie para soportar el peso corporal en la fase de apoyo; 2) la liberación del pie que no mantiene el apoyo durante la fase de oscilación; 3) el apropiado posicionamiento del pie durante la última parte de la fase de oscilación prara el apoyo y el comienzo del siguiente ciclo; y 4) una adecuada longitud de paso.

Los movimientos coordinados del tobillo, la rodilla, la cadera y la pelvis tienen, por tanto, como objetivo atenuar, mediante la amortiguación, la brusquedad del movimiento rítmico de los pies y conservar la suavidad de la curva sinusoidal del centro de gravedad. La marcha es una acción realizada por tres segmentos que actúan de forma coordinada (miembros inferiores y pelvis), por lo que la utilización de únicamente los miembros inferiores da una marcha en “tijera” en la que, evidentemente, faltaría un elemento (Neumann, 2002; Viel, \& Plas, 2002).

Durante el ciclo de la marcha la pelvis describe un movimiento sincronizado y de poca amplitud en los tres planos del espacio. En el plano sagital, se observa una anteversión en posición estática de aproximadamente $10^{\circ}$ que aumentará otros $4^{\circ}$ durante el ciclo de la marcha. En el plano frontal, la pelvis presenta un movimiento de basculación lateral de $4^{\circ}$ hacia el lado de la extremidad oscilante durante la fase previa a la oscilación, siendo el rango total de movimiento en este plano de $8^{\circ}$. La función principal de la inclinación lateral de la pelvis es el reducir el movimiento vertical ascendente del centro de gravedad durante el apoyo monopodal. Además, en el plano transversal se observa una rotación aproximada de $10^{\circ}, 5^{\circ}$ hacia delante durante la fase terminal de la oscilación y el contacto inicial, y $5^{\circ}$ hacia atrás en la fase terminal de la fase de apoyo (Perry, \& Burnfield, 2010). Esta acción denominada por Viel y Plas (2002) "paso pélvico" se da en el mismo sentido del pie que avanza y es la que permite dar amplitud al paso (Figura 6).

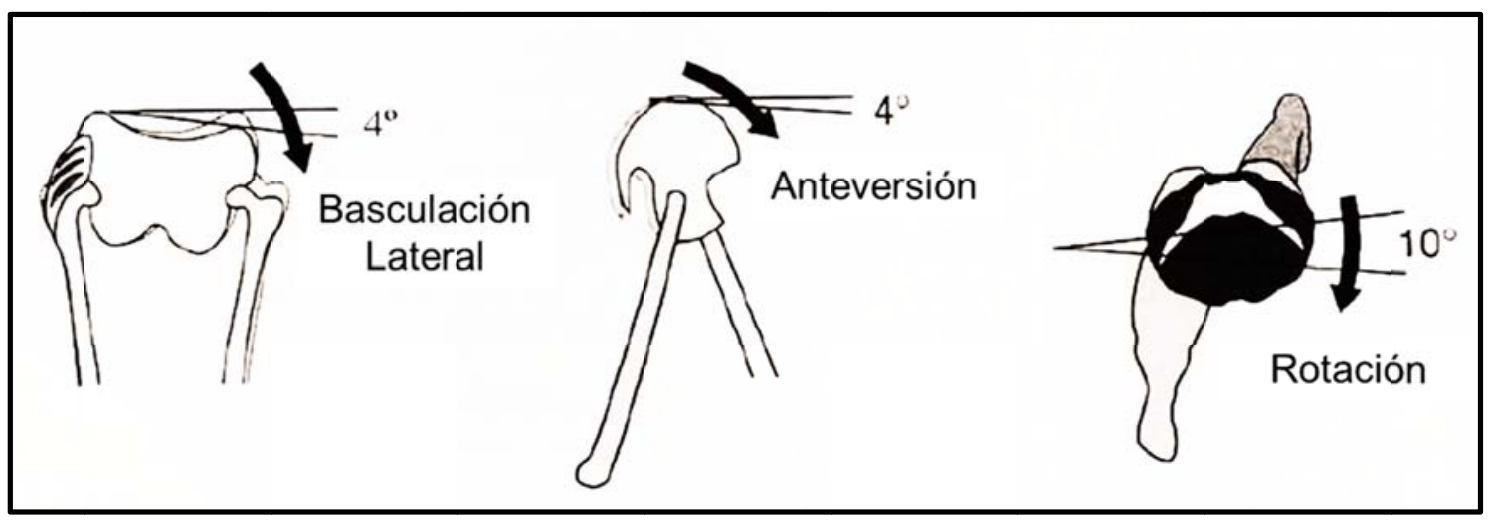

Figura 6. Movimientos de la pelvis durante el ciclo de la marcha (adaptado de Perry, 1992).

La cadera en el plano sagital describe dos curvas de movimiento, que son la extensión en la fase de apoyo y la flexión durante la fase de oscilación, con un rango total de movimiento de 


\section{INTRODUCCIÓN}

entre $40^{\circ}$ y $50^{\circ}$ (Neumann, 2002; Perry, \& Burnfield, 2010). En el contacto inicial, la cadera se encuentra en $30^{\circ}$ de flexión y se mantiene de igual forma hasta el final de la fase de amortiguación (10\% del ciclo). A partir de ese momento, y durante la fase media de apoyo y la fase terminal de apoyo, se producirá una extensión progresiva y continua de la cadera hasta alcanzar aproximadamente -10 grados de extensión en el 50\% del ciclo. Durante la fase previa a la oscilación y la fase inicial de oscilación, se produce una flexión, también progresiva y continua, hasta alcanzar los $25^{\circ}$ al final de la fase inicial de oscilación. En la fase media de oscilación, la cadera continuará flexionándose hasta los $40^{\circ}$ alcanzando su máximo de flexión en el ciclo. En la última fase del ciclo, la fase terminal de oscilación, se producirá una leve extensión hasta alcanzar los $30^{\circ}$ de flexión de cadera para el contacto del pie con el suelo (Figura 7).

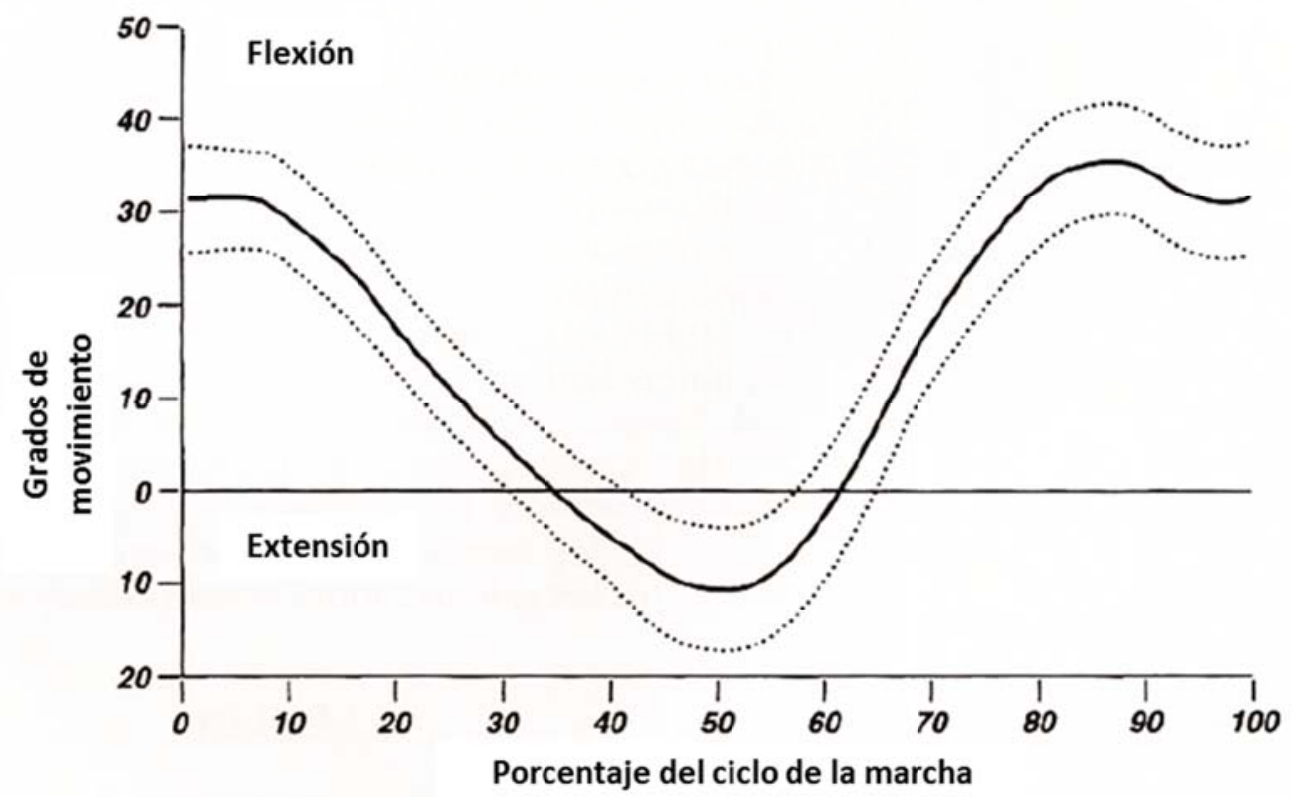

Figura 7. Movimiento de flexo-extensión de la cadera durante el ciclo de la marcha (plano sagital) (adaptado de Perry, 1992).

La rodilla muestra cuatro curvas de movimiento en el plano sagital (flexo-extensión) durante el ciclo de la marcha. En el instante del contacto inicial del talón la rodilla se encuentra en una postura media de $5^{\circ}$ de flexión, pudiendo variar desde la extensión completa o $0^{\circ}$ de flexión hasta los $10^{\circ}$ de flexión. El primer pico de flexión $\left(20^{\circ}\right)$ se encuentra en el momento de absorción del impacto entre el contacto inicial y la fase media de apoyo (aproximadamente alrededor del $10 \%$ del ciclo). Desde ese momento, se produce una extensión que alcanzará el máximo en la mitad de la fase terminal del apoyo (39\% del ciclo) y comenzará a flexionar nuevamente alcanzando en torno a $40^{\circ}$ en el instante del despegue del pie. El segundo pico 
( $60^{\circ}$ de flexión) se presenta en la fase inicial de oscilación ( $72 \%$ del ciclo) y será la máxima flexión alcanzada por la rodilla durante el ciclo de la marcha. A partir de ese momento, se produce una extensión rápida hasta $0^{\circ}$ en el $95 \%$ del ciclo y una pequeña flexión de $5^{\circ}$ en el 100\% para el contacto del talón con el suelo (Perry, \& Burnfield, 2010) (Figura 8).

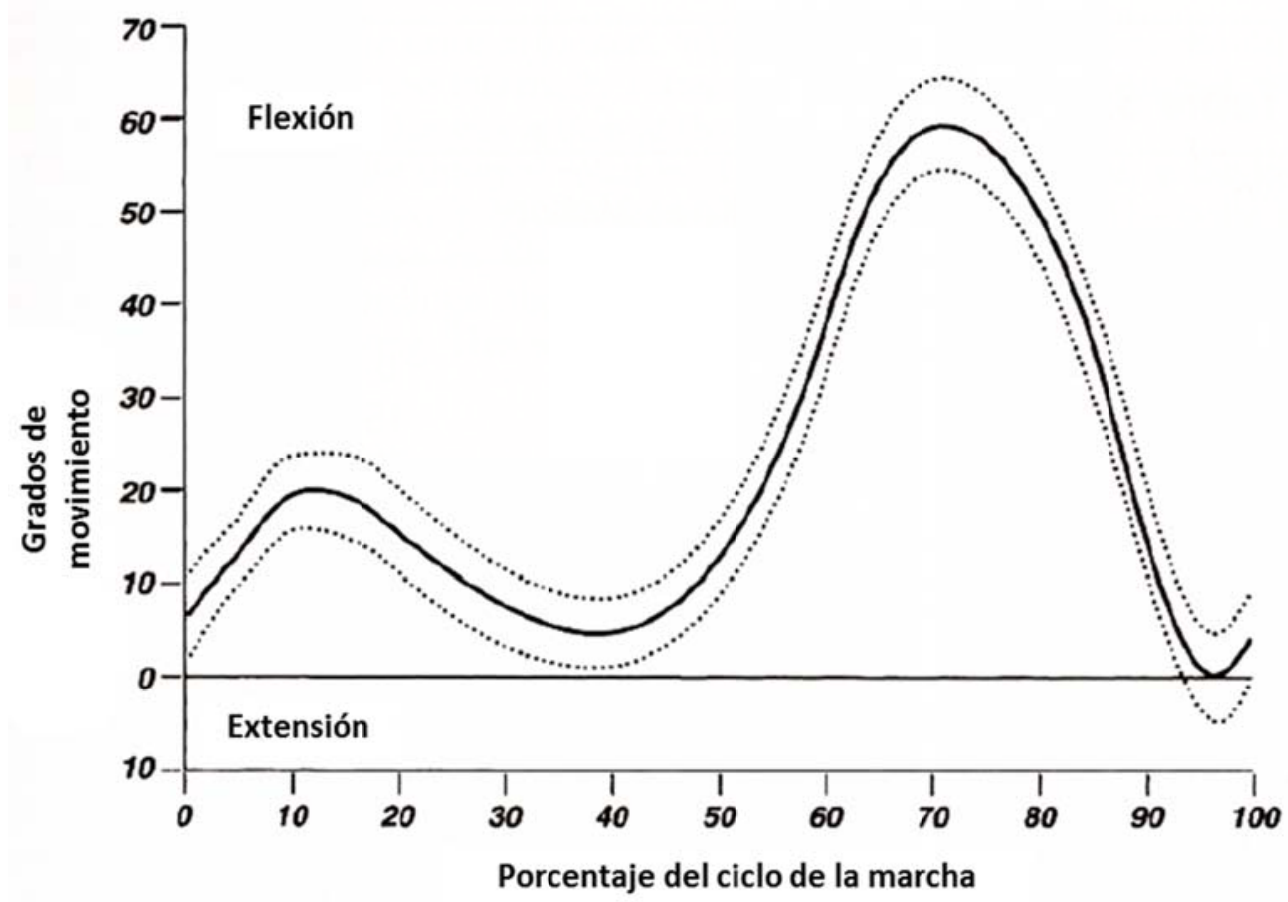

Figura 8. Movimiento de flexo-extensión de la rodilla durante el ciclo de la marcha (plano sagital) (adaptado de Perry, 1992).

El tobillo también muestra cuatro curvas de movimiento en el plano sagital (flexo-extensión) durante el ciclo de la marcha. Durante la fase de apoyo, hasta el $60 \%$ del ciclo, se encuentran los tres primeros arcos (extensión-flexión-extensión). Desde el contacto inicial hasta la mitad de la fase de amortiguación (5\%), se produce una extensión de $-5^{\circ}$ del tobillo a consecuencia de la caída de la parte anterior del pie. Desde ese momento, y de manera progresiva, se produce una flexión del tobillo a medida que la tibia avanza en relación al pie situado en el suelo. La máxima flexión de tobillo $\left(10^{\circ}\right)$ se alcanza en torno al $45 \%$ del ciclo de la marcha y se mantiene hasta el final de la fase terminal de apoyo. A continuación, durante la fase previa de oscilación (50\%-60\%) se produce una extensión rápida del tobillo pasando de los $10^{\circ}$ de flexión a $-15^{\circ}$ de extensión. Ya en la fase de oscilación se produce una nueva flexión del tobillo que alcanza su máximo en el $75 \%$ del ciclo ( $5^{\circ}$ de flexión) y vuelve a la extensión (- $2^{\circ}$ de extensión) en el 100\% (Perry, \& Burnfield, 2010) (Figura 9). 


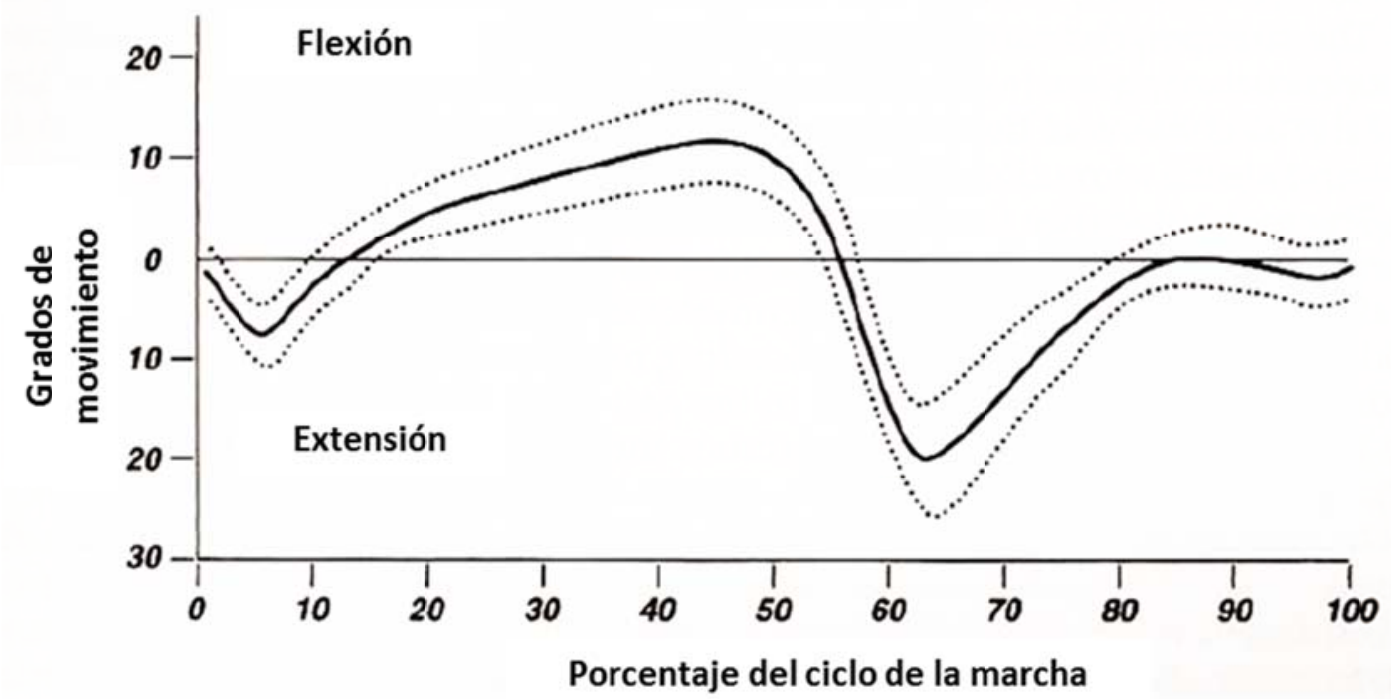

Figura 9. Movimiento de flexo-extensión del tobillo durante el ciclo de la marcha (plano sagital) (adaptado de Perry, 1992).

\subsubsection{Alteraciones de la Marcha en Patologías}

La marcha humana se ve frecuentemente afectada o influenciada por un gran número de patologías del sistema neuromusculoesquelético. Aunque cada una de ellas determinan patrones mecánicos diferentes de la marcha según sus características, se pueden clasificar en cinco categorías funcionales: deformidad, debilidad muscular, pérdida de sensibilidad, dolor y déficit de control motor (Perry, \& Burnfield, 2010).

La deformidad funcional se presenta cuando los tejidos no permiten una postura y rango de movimiento adecuado y suficiente para la marcha normal. Esto puede deberse a contracturas musculares, que condicionarán también el funcionamiento de ligamentos y cápsulas articulares, contornos articulares anormales, como la artrosis, o defectos congénitos, como el pie equinovaro. Un ejemplo de este grupo de patologías, se podría encontrar en la rodilla bloqueada en flexión por contracturas musculares, que impide, durante la fase de apoyo, la extensión completa e implica una mayor actividad muscular para estabilizar la rodilla durante el momento de soporte del peso corporal. Además, se impedirá también la extensión de la rodilla durante la fase final de oscilación, antes del contacto del talón con el suelo, por lo que la longitud de paso se verá reducida (Perry, \& Burnfield, 2010).

La debilidad muscular, por atrofia muscular por desuso o por patologías neurológicas, implica que la persona genera una fuerza muscular insuficiente para las demandas de la marcha normal. Los pacientes que únicamente muestran debilidad muscular por miopatías (poliomelitis, Guillain-Barré, distrofia muscular, etc.) y que mantienen un adecuado control motor, son capaces de sustituir y modificar la actividad muscular para evitar posturas 
inadecuadas o una mala alineación de los segmentos durante la fase de apoyo, así como para el avance de la pierna durante la fase de oscilación. Además, la elección de una velocidad de la marcha más lenta se traduce en una menor demanda muscular (Perry, \& Burnfield, 2010; Prat Pastor, 1999).

La pérdida de sensibilidad y, por tanto, una deficiente capacidad de propiocepción, conlleva la falta de conocimiento de las posiciones articulares en el espacio y del instante de contacto del talón con el suelo. Se observarán patrones de la marcha con rodillas bloqueadas en extensión y golpeos exagerados del talón en el suelo durante el contacto inicial, como forma de compensar la falta de propiocepción. También en este tipo de patologías los pacientes seleccionan una velocidad más lenta durante la marcha (Perry, \& Burnfield, 2010).

Pacientes con dolor en el sistema neuromusculoesquelético (traumatismos, artritis, etc.) muestran fundamentalmente deformidad funcional y debilidad muscular en las articulaciones afectadas, factores limitantes de la marcha normal descritos anteriormente. La deformidad funcional como consecuencia de la adquisición de posturas antiálgicas implica menor rango articular de movimiento, menor tensión en tejidos periarticulares, menor presión intraarticular; y la debilidad muscular por inactividad debida a distensión de los tejidos o derrame articular (Perry, \& Burnfield, 2010; Prat Pastor, 1999).

El control motor puede verse alterado por cualquier mecanismo lesional que afecte al cerebro o a las regiones cervical y torácica de la médula espinal, como por ejemplo el accidente cerebrovascular, traumatismo craneoencefálico, tetraplejia, paraplejia, esclerosis múltiple, parálisis cerebral, hidrocefalia, infecciones o tumores. La falta de control motor implica cuatro déficits funcionales que se combinan de manera diferente y con intensidad variable; la debilidad muscular, el deterioro del control selectivo, la aparición de patrones motores primitivos y la espasticidad. Las consecuencias de estos déficits funcionales en la marcha se reflejan fundamentalmente en la pérdida de control de intensidad y duración de la contracción muscular concéntrica y excéntrica. Así como la sustitución, dependiendo del control selectivo y propiocepción remanente, de los patrones normales por patrones primitivos menos desarrollados y que no permiten la realización simultánea de flexión y extensión de las diferentes articulaciones (Perry, \& Burnfield, 2010).

\subsubsection{Otros Factores que Modifican el Patrón de la Marcha}

Además de las patologías del sistema neuromusculoesquelético que influyen y modifican el patrón de la marcha, existen otros factores biomecánicos y anatómicos que condicionan la forma de caminar de cada individuo. El sexo, las características antropométricas o la velocidad a la que se camina, por condiciones socio-culturales (Bruening, Frimenko, Goodyear, Bowden, 
\& Fullenkamp, 2015) o por adaptaciones evolutivas (Troje, 2002), determinan el patrón de la marcha de cada sujeto.

\subsubsection{Influencia del sexo y estatura en el patrón de la marcha}

El término "marcha normal" descrito como estándar engloba a patrones de ambos sexos y a un amplio rango de características antropométricas individuales que influyen y determinan el patrón de la marcha. La estatura condiciona la longitud de paso y la cadencia y, por tanto, determina también la velocidad. Hof (1996) y Pierrynowski y Galea (2001) señalan que las personas con estaturas más bajas seleccionan una longitud de paso menor y una cadencia mayor para conseguir la misma velocidad que las personas con mayor estatura. También indican, junto a otros autores (Frimenko, Goodyear, \& Bruening, 2015; Hof, 1996; Kirtley, 2006; Pierrynowski, \& Galea, 2001), la importancia de la normalización de los parámetros espaciotemporales de la marcha influenciados por la estatura. De este modo se representan y caracterizan los patrones disminuyendo las características propias de las diferencias antropométricas de los sujetos. La velocidad, longitud de paso y longitud de zancada se normalizan en función de la estatura y de la longitud de pierna, y la anchura de paso en función de la anchura de la pelvis, determinada por la distancia entre las espinas ilíacas anterosuperiores (EIAS).

El estudio de la influencia del sexo en los patrones de la marcha y las diferencias entre hombres y mujeres han sido descritas desde los años 60 en los estudios de Murray y colaboradores (Murray, Drought, \& Kory, 1964; Murray, Kory, \& Sepic, 1970) que caracterizaban los patrones de la marcha normal de hombres y mujeres por separado. Las mujeres en comparación con los hombres presentan una velocidad de la marcha menor (Chiu, \& Wang, 2007; Cho, Park, \& Kwon, 2004; Crosbie, Vachalathiti, \& Smith, 1997; Kerrigan, Todd, \& Della Croce, 1998), una longitud de paso o de zancada menor (Bruening et al., 2015; Cho et al., 2004; Frimenko et al., 2015; Kerrigan et al., 1998) y una cadencia mayor (Bruening et al., 2015; Crosbie et al., 1997; Frimenko et al., 2015; Kirtley, 2006). En los parámetros cinemáticos de cadera, rodilla, tobillo, pelvis y centro de gravedad, también se muestran características propias de cada sexo. Las mujeres presentan mayor flexión de cadera y rodilla (Cho et al., 2004; Nigg, Fisher, \& Ronsky, 1994; Røislien, Skare, Gustavsen, Broch, Rennie, \& Orpheim, 2009) y una mayor flexión plantar de tobillo (extensión) durante el despegue del pie (Chiu, \& Wang, 2007; Kerrigan et al., 1998; Røislien et al., 2009). Sin embargo, es la basculación lateral de la pelvis en el plano frontal, mayor en las mujeres, y el movimiento lateral del centro de gravedad, mayor en los hombres, son lo que numerosos autores describen (Bruening et al., 2015; Mather, \& Murdoch, 1994; Murray et al., 1964; Murray et al., 1970; Smith, Lelas, \& 
Kerrigan, 2002; Troje, 2002) como los mayores discriminantes entre patrones de la marcha de hombres y mujeres. Por tanto, el estándar "normal" de la marcha dependerá de las características antropométricas y el sexo de cada sujeto. Sería apropiado entonces, al evaluar cualquier patrón individual, comparar con el estándar de un grupo de población con las mismas características del sujeto estudiado.

\subsubsection{Influencia de la velocidad en el patrón de la marcha}

La velocidad normal de la marcha para personas adultas y sin patologías se encuentra en un rango de confort de entre $1,0 \mathrm{~m} / \mathrm{s}$ y $1,5 \mathrm{~m} / \mathrm{s}$ (Bohannon, \& Andrews, 2011; Kirtley, 2006; Nymark, Balmer, Melis, Lemaire, \& Millar, 2005; Pépin, Ladouceur, \& Barbeau, 2003; Perry, \& Burnfield, 2010). Bohannon y Andrews (2011) en su revisión "Normal walking speed" concretaron incluso una velocidad normal para adultos sanos de 1,34 a 1,43 m/s para los hombres, y de 1,24 a $1,39 \mathrm{~m} / \mathrm{s}$ para las mujeres.

Estudios previos han determinado que pacientes con patologías del sistema nervioso o musculo-esquelético eligen desplazarse espontáneamente a velocidades más lentas de 1,0 m/s (Tesio, Lanzi, \& Detrembleur, 1998). Pacientes con hemiplejia tras accidente cerebrovascular (Bohannon, 1987; Onley, \& Richards, 1996), pacientes con osteoartritis (Bejek, Paróczai, Illyés, \& Kiss, 2006) o personas de avanzada edad (Bohannon, Andrews, \& Thomas, 1996) eligen velocidades lentas, en torno a 0,40 y 0,80 m/s. Pacientes con lesión medular incompleta que mantienen una función motora y sensitiva residual que les permite caminar eligen velocidades extremadamente lentas, entre 0.20 y $0.30 \mathrm{~m} / \mathrm{s}$ dependiendo del nivel de discapacidad (Krawetz, \& Nance, 1996; Melis, Torres-Moreno, Barbeau, \& Lemaire, 1999; Nymark et al., 2005; Pépin, Norman, \& Barbeau, 2003; Pépin et al., 2003b; Waters, Yakura, Adkins, \& Barnes, 1989).

Caminar a velocidades lentas implica mayor esfuerzo para el mantenimiento del equilibrio debido al aumento del movimiento medio-lateral del centro de gravedad y de la anchura de paso; no es una marcha más eficiente, pero algunos pacientes la eligen por ser una marcha más segura con la que se previenen las caídas (Orendurff, Segal, Klute, Berge, Rohr, \& Kadel, 2004). En otras ocasiones, la elección viene determinada por aspectos anatómicos que condicionan la cinemática de las articulaciones y la imposibilidad de aumentar la longitud de paso y la cadencia y, por tanto, la velocidad (Pépin et al., 2003b).

Numerosos autores han estudiado la influencia de la velocidad en los parámetros espaciotemporales y cinemáticos, determinando cuáles son los cambios que se producen en la marcha humana durante una velocidad lenta (Beauchet et al., 2009; Bejek et al., 2006; Chiu, Wu, \& Chang, 2013; Chung, \& Wang, 2010; Den Otter, Geurts, Mulder, \& Duysens, 2004; Hirasaki, 
Moore, Raphan, \& Cohen., 1999; Jordan, Challis, \& Newell, 2007; Kang, \& Dingwell, 2007; Kavanagh, 2009; Nymark et al., 2005; Orendurff et al., 2004; Pépin et al., 2003a; Pépin et al., 2003b; Riley, Della Croce, \& Kerrigan, 2001; Shemmell, Johansson, Portra, Gottlieb, Thomas, \& Corcos, 2007; Swinnen et al., 2013; Tanawongsuwan, \& Bobick, 2003; Tesio et al., 1998; Thomas, De Vito, \& Macaluso, 2007; Yoon, Park, \& Damiano, 2012). La cadencia y longitud de paso o longitud de zancada, directamente relacionados con la velocidad, disminuyen de forma paralela a la velocidad (Bejek et al., 2006; Kirtley, 2006; Riley et al., 2001; Shemmell et al., 2007; Tanawongsuwan, \& Bobick, 2003), mientras que el ancho de paso se incrementa para dar mayor base de sustentación (Ordendurff et al., 2004). El aumento del tiempo de apoyo simple y de apoyo doble y, por lo tanto, el aumento de la proporción de la fase de apoyo en el ciclo es una de las modificaciones más características en el patrón de la marcha a velocidad lenta (Baker, 2013; Bejek et al., 2006; Kirtley, 2006; Shemmell et al., 2007). En los parámetros cinemáticos, también se muestran diferencias, fundamentalmente en el movimiento del centro de gravedad, que disminuye sustancialmente en el plano sagital (movimiento ascendente y descendente) y aumenta en el plano frontal (movimiento lateral), y en la flexoextensión de cadera, rodilla y tobillo. La cadera y la rodilla disminuyen su rango de movimiento en las velocidades lentas, mostrando menor pico de flexión y extensión; mientras que el tobillo presenta una mayor dorsiflexión (flexión) en el apoyo del talón y menor flexión plantar (extensión) durante el despegue del pie (Baker, 2013; Bejek et al., 2006; Ordendurff et al., 2004; Riley et al., 2001; Swinnen et al., 2013).

Poniendo de manifiesto los cambios en el patrón de la marcha como consecuencia de una disminución de la velocidad, resulta importante el estudio de los patrones de la marcha con sujetos sanos y la creación de bases de datos a velocidades lentas con el objetivo de poder disociar las modificaciones del patrón normal relativas a las patologías neuromusculoesqueléticas de las adaptaciones producidas por la velocidad (Den Otter et al., 2004; Nymark et al., 2005). Además, estas bases de datos debieran estar diseñadas para cada una de las velocidades elegidas en cada una de las patologías. Por ese motivo, y según los datos señalados anteriormente, en el presente estudio se optó por $0.25 \mathrm{~m} / \mathrm{s}$ como la velocidad idónea para la reproducción de los patrones en el exoesqueleto, por ser la velocidad seleccionada por los pacientes con lesión medular incompleta que mantienen una función motora y sensitiva residual que les permite caminar, y principales usuarios de este tipo de dispositivos de rehabilitación (Krawetz, \& Nance, 1996; Melis et al., 1999; Nymark et al., 2005; Pépin et al., 2003a; Pépin et al., 2003b; Waters et al., 1989).

El describir y analizar las diferencias que se encuentran cuando se disminuye la velocidad de la marcha en el presente estudio se debe, principalmente, a la necesidad de identificar el patrón 
que será reproducido por el exoesqueleto y que se utilizará para la rehabilitación y compensación de la marcha. Con ello se determina si al caminar a $0.25 \mathrm{~m} / \mathrm{s}$ se realizan cambios sustanciales en el patrón de la marcha que condicionen la cinemática de las articulaciones, y se establece la idoneidad del patrón lento para la rehabilitación de la marcha.

\subsubsection{Instrumentos de Valoración de la Marcha}

Desde finales del siglo XIX, la fotografía ha sido principalmente el método utilizado para el análisis cinemático del movimiento humano y animal, a través de fotografías secuenciales y fotografías con sobreexposición (Figura 10). Hoy en día pocos laboratorios de análisis de la marcha continúan manteniendo este tipo de técnicas por el tiempo y esfuerzo manual que requiere el procesamiento de los datos, aunque siguen siendo utilizadas, remplazándolas por grabación en video de alta velocidad, en la captura de actividades deportivas, gracias a las posibilidades que ofrece de grabación en el campo de juego y no exclusivamente en entornos de laboratorio (fotogrametría) (Whittle, 2007).

Otros métodos utilizados son los goniómetros electrónicos, que, probablemente, son el método más sencillo de obtener información cinemática del movimiento y que, aunque son relativamente baratos y ofrecen mediciones en tiempo real, tienen como principal limitación la necesidad de una alineación exacta de su eje de giro con el de la articulación. Además, únicamente ofrecen medidas bidimensionales relativas de rangos de movimientos articulares y no el movimiento absoluto de los segmentos en el espacio (Kirtley, 2006; Perry, \& Burnfield, 2010). Por otra parte, los sensores inerciales ofrecen un método más económico de medir aceleraciones y velocidades angulares a través de acelerómetros, giroscopios o magnetómetros, $y$, aunque tienen la gran ventaja de poder ser utilizados en cualquier medio, no ofrecen unas mediciones tan precisas como los sistemas optoelectrónicos.

La gran mayoría de los sistemas de captura de movimiento comercializados y utilizados en laboratorios de análisis de la marcha son sistemas optoelectrónicos tridimensionales que permiten la obtención de la posición y orientación de los segmentos, ángulos articulares, velocidades lineales y angulares, y aceleraciones durante el análisis cinemático. Para ello es necesaria la grabación simultánea de más de dos cámaras y la calibración del espacio de captura con un sistema de referencia tridimensional. 

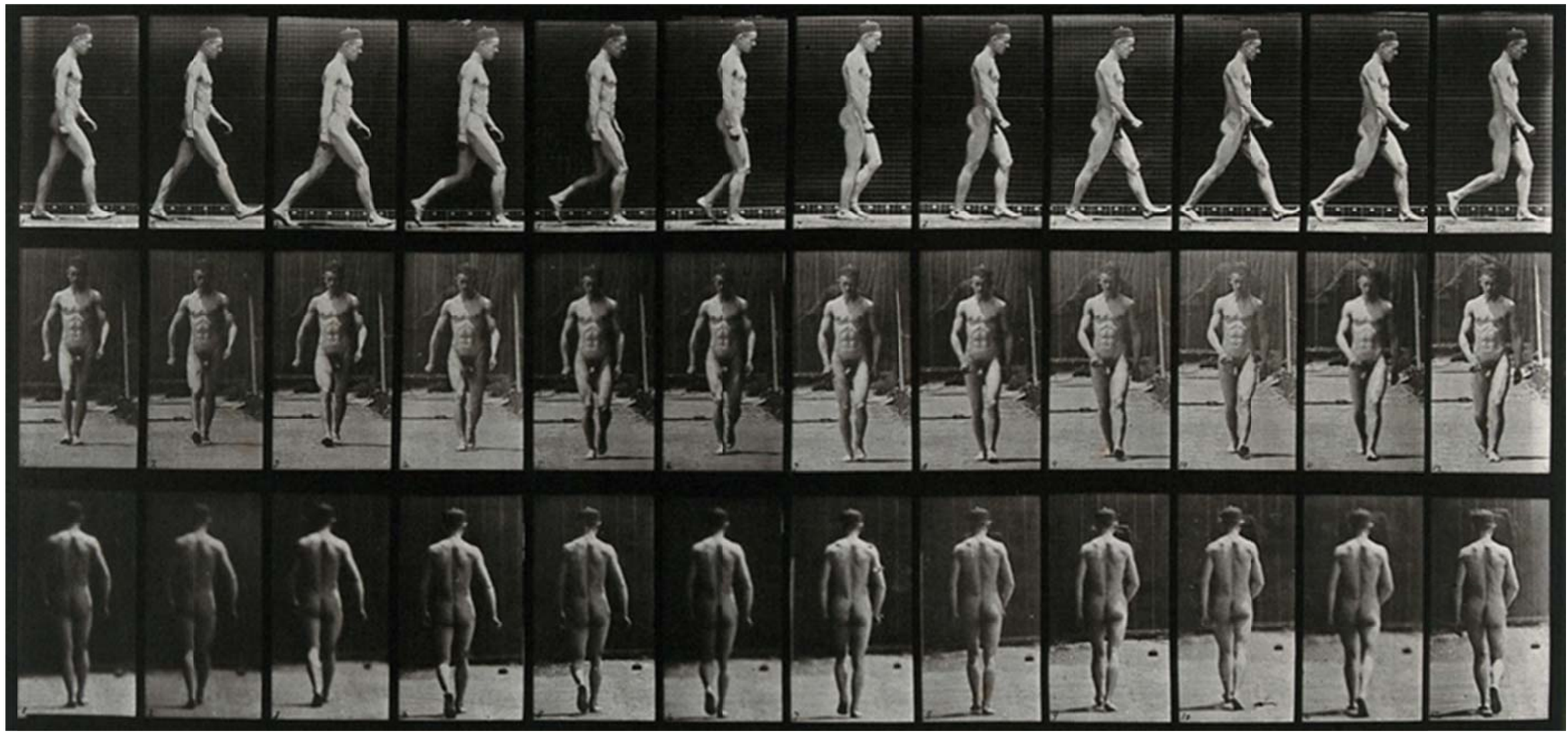

Figura 10. Estudio de la Marcha por Eadweard Muybridge (1955). 
Este tipo de sistemas optoelectrónicos incluyen métodos de calibración automática durante la que se calcula la relación entre marcadores reflectantes conocidos previamente y su posición bidimensional en el espacio de captura por cada cámara (VICON Motion Systems, Optotrak, BTS, etc.) (Figura 11) (Kirtley, 2006; Whittle, 2007). Normalmente existen dos tipos de calibración que se realizan de forma secuencial: la calibración estática, utilizada para establecer el origen de coordenadas y la dirección de los ejes en el espacio, y la calibración dinámica, que determina la posición relativa y orientación de las cámaras.

Una vez que el sujeto camina en el espacio de captura calibrado se obtendrán las posiciones tridimensionales de los marcadores localizados en puntos anatómicos del sujeto, siempre y cuando éstos sean reconocidos por al menos dos cámaras de forma simultánea. Si esto no sucediese, por oclusión, la posición tridimensional del marcador no podría calcularse y sería estimada por interpolación teniendo en consideración la posición previa y posterior al instante no registrado. De esta forma, con la secuencia de datos recogidos, se reconstruye la trayectoria tridimensional de cada uno de los marcadores reflectantes. Todos los datos son recogidos en intervalos de tiempo, denominados fotogramas, con una frecuencia de grabación que, dependiendo de los sistemas, puede oscilar entre $50 \mathrm{~Hz}$ y $500 \mathrm{~Hz}$ (Kirtley, 2006; Perry, \& Burnfield, 2010; Whittle, 2007).

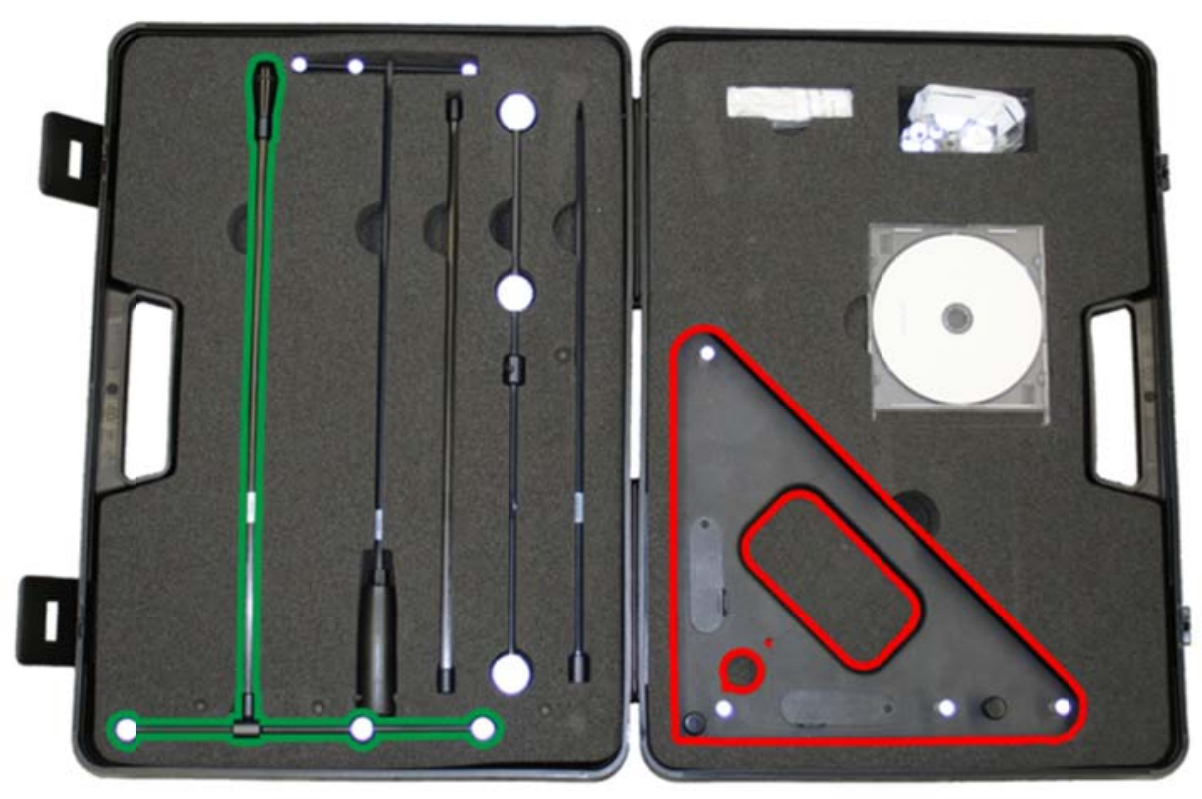

Figura 11. Sistema de marcadores para la calibración: kit de calibración de VICON. En verde "Calibration Wand" para la calibración dinámica y en rojo "L-Frame" para la calibración estática (tomado de Motion Capture Studio, 2009). 
La colocación de marcadores reflectantes sobre el cuerpo del sujeto está determinada por modelos tridimensionales en los que al menos tres marcadores definen la posición de cada segmento, y el movimiento de dos segmentos adyacentes a una articulación definen el movimiento articular. Los marcadores se sitúan sobre superficies anatómicas exactas siguiendo las directrices de cada modelo. Por ejemplo, en el modelo modificado de Helen Hayes Model el segmento pierna se definirá por los marcadores situados en tobillo, rodilla y parte media de la pierna (Figura 12). Ejemplos de estos modelos son el modelo modificado de Helen Hayes Model, Vaughan, Newington, Kadaba, Davis, Gage o Vicon Clinical Manager (Kirtley, 2006). En el apartado "2. Metodología" se desarrollará el modelo concreto utilizado para las mediciones del presente estudio (Plug in Gait Model de VICON).

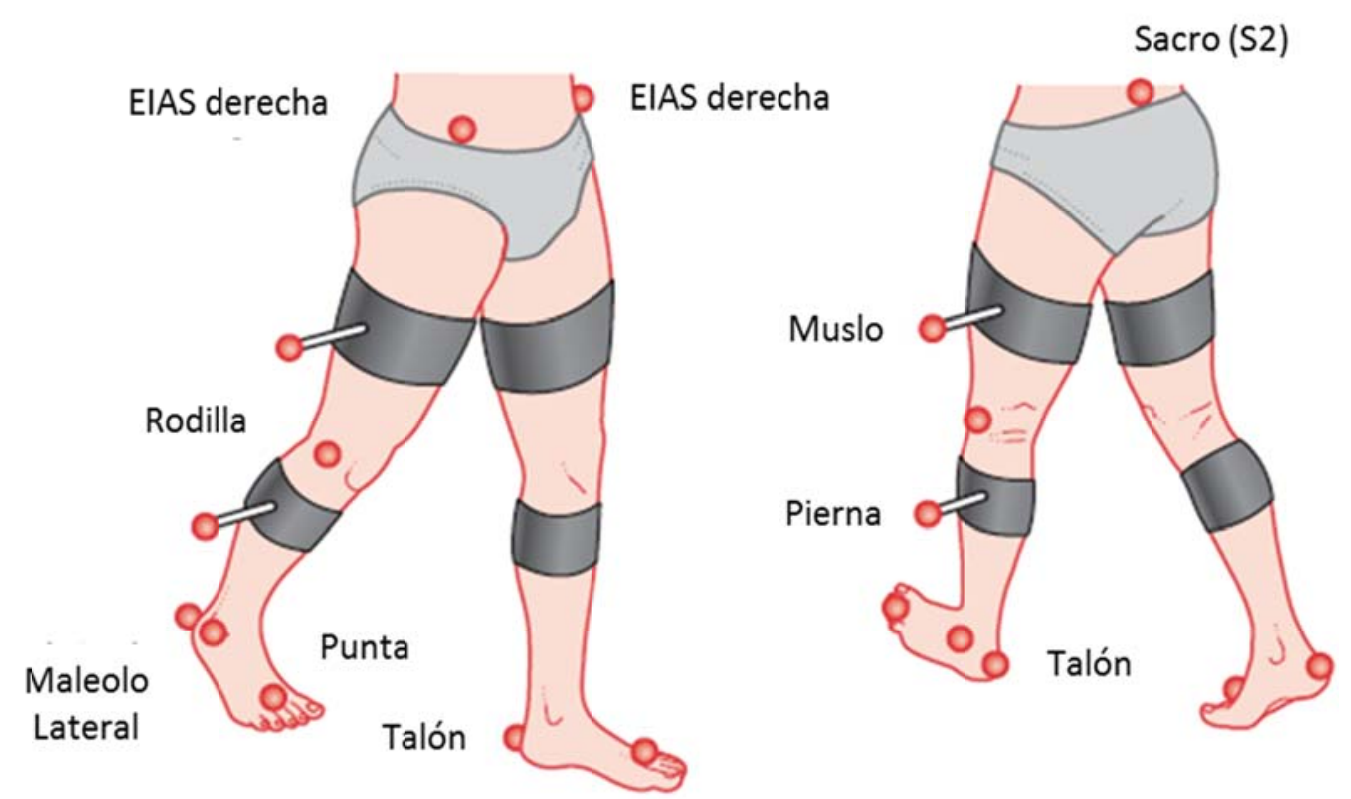

Figura 12. Modelo para la colocación de marcadores modificado de Helen Hayes Model (adaptado de Kirtley, 2006).

El Laboratorio de Biomecánica Deportiva de la Facultad de Ciencias de la Actividad Física y el Deporte (Universidad Politécnica de Madrid) dispone de un sistema de análisis de movimiento en tres dimensiones VICON (Vicon Motion System, Oxford, UK), compuesto por seis cámaras con iluminación LED infrarroja (Figura 13). La frecuencia de grabación de estas cámaras puede oscilar entre 50 y $500 \mathrm{~Hz}$, entre 50 fotogramas/s a 500 fotogramas/s, lo que dependerá del movimiento a analizar. 

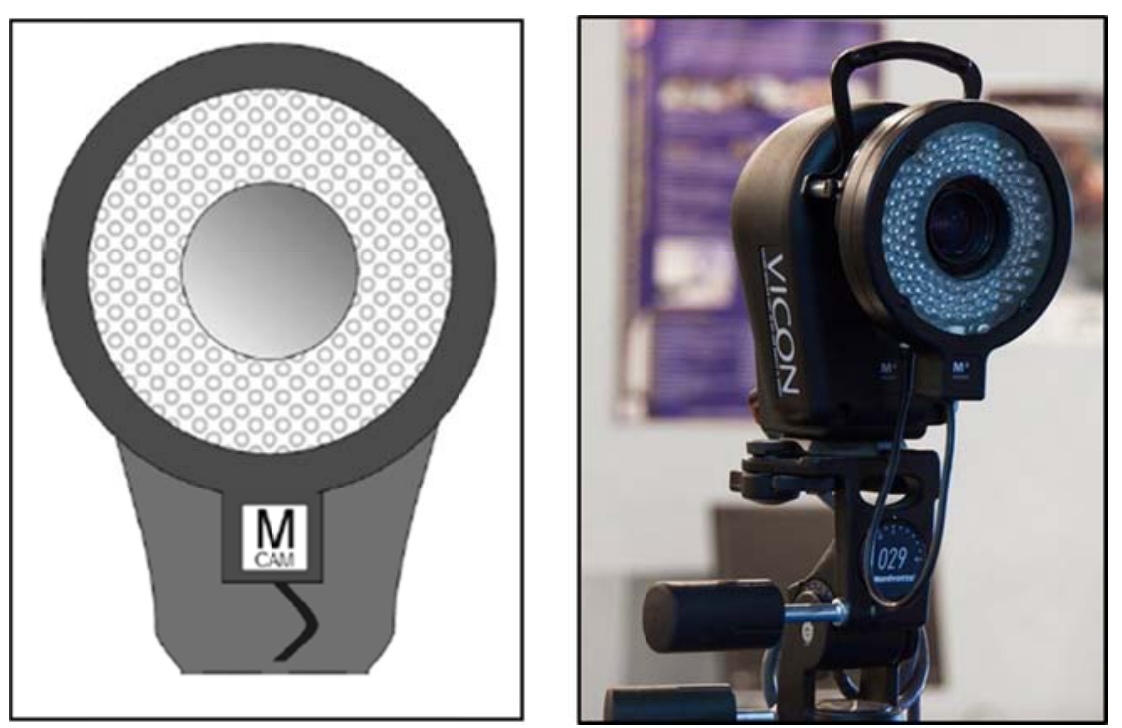

Figura 13. Cámara de sistema VICON. De izquierda a derecha: Esquema de la disposición de LEDs alrededor de la lente (tomado de Vicon, 2003) y fotografía de una cámara.

El sistema incluye un software específico para la captura de movimiento, edlición y procesado de los datos, así como para la creación de modelos cinemáticos propios para el análisis biomecánico y la animación (Workstation, BodyBuilder y Polygon) (Vicon, 2003). Además, está preparado para la sincronización con otros dispositivos como plataformas de fuerza, electromiografos, y captura de audio y video. Se completa con una serie de marcadores esféricos reflectantes, con $14 \mathrm{~mm}$ de diámetro, que reflejan la luz infrarroja emitida por las cámaras y devuelven una imagen en forma de esferas brillantes que es captada y grabada por las cámaras (Figura 14).

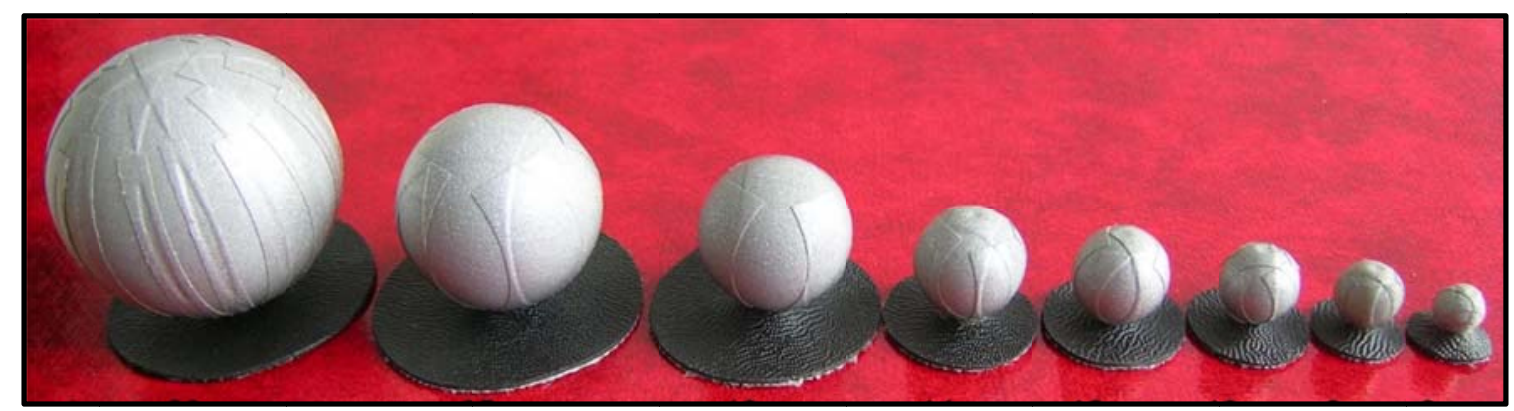

Figura 14. Marcadores reflectantes esféricos (tomado de ZFlo Motion Analysis Systems, 2015).

\subsubsection{Aplicaciones del Análisis de la Marcha}

Las aplicaciones del análisis de la marcha podrían dividirse en dos amplias categorías: la valoración clínica de la marcha y la investigación de la marcha. La evaluación clínica tiene como objetivo ayudar de forma directa e individual a pacientes, mientras que la investigación 


\section{INTRODUCCIÓN}

mejora la comprensión del comportamiento y los parámetros de la marcha, que a su vez puede aplicarse a la mejora del diagnóstico y tratamiento médico (Whittle, 2007).

Davis, Ounpuu, Tyburski y Gage (1991), describían el análisis de la marcha como una medición sistematizada, descripción y evaluación de los valores que caracterizan la locomoción humana y justificaban el análisis de la marcha, en una aplicación clínica, como una herramienta de evaluación de los trastornos de la marcha. La principal aplicación y prioridad en la actualidad del análisis de la marcha en la valoración clínica es la utilización como herramienta en el proceso de toma de decisiones en el diagnóstico como forma de mejorar la prescripción de tratamiento de los pacientes, como describe la Gait Clinical Movement Analysis Society (GCMAS) en su evaluación de las prioridades en el análisis de la marcha realizado por su comité científico en 2007 (Theologis, 2009). Además, el análisis de la marcha ofrece una herramienta de control y monitorización durante y posterior al tratamiento (terapia física, cirujía, intervenciones con órtesis y prótesis o medicamentos) (Kirtley, 2006). La investigación en el ámbito del análisis de la marcha se centra, por un lado, en el conocimiento de los trastornos patológicos y los métodos de tratamiento, y, por otro lado, en el desarrollo de métodos de medida y mejora del conocimiento en la biomecánica, el rendimiento humano y la fisiología (Whittle, 2007).

Un gran número de trastornos neuromusculoesqueléticos que producen alteraciones en la marcha se benefician del análisis clínico como forma de mejora del diagnóstico y adecuación de los tratamientos específicos: parálisis cerebral, Parkinson, distrofia muscular, osteoartritis, artritis reumatoide, amputaciones de miembro inferior, accidentes cerebrovasculares, traumatismos craneoencefálicos, lesión medular, mielodisplasia o esclerosis múltiple. Y otros como la adecuación de las cirugías de remplazo articular, la cirugía y rehabilitación de roturas del ligamento cruzado de rodilla, la planificación de tratamiento en hemiplejias, el control de escaras de presión en el pie diabético o la valoración de la alineación y ajuste de órtesis y prótesis. Además, grupos de población, como las personas con edad avanzada, pueden mejorar su calidad de vida reduciendo el riesgo de caídas y fracturas gracias a la identificación de los factores que producen los trastornos de la marcha como parte del proceso normal de envejecimiento (Whittle, 2007). 


\subsection{Lesión Medular}

\subsubsection{Causas de Lesión Medular: Epidemiología}

La Asociación Nacional de Lesionados Medulares y Grandes Discapacitados Físicos (ASPAYM) en su "Análisis sobre la lesión medular en España" realizado en 2012 (ASPAYM, 2012) define la lesión medular como la pérdida o alteración de la movilidad, de la sensibilidad o del sistema nervioso autónomo ocasionada por un trastorno de las estructuras nerviosas alojadas en el canal medular. En España, la lesión de médula espinal es una de las deficiencias más importantes producida mayoritariamente por causas traumáticas. Aproximadamente cada año se producen unos 1000 casos nuevos de lesión medular, lo que asciende el número total de afectados a 30.000. Las lesiones pueden deberse a un origen traumático, por accidentes de tráfico (38.5\% de los casos), accidentes laborales o caídas desde altura, actividades deportivas e intentos de suicidio; o a un origen médico (alrededor de un $30 \%$ de los casos) debidas a distintas enfermedades (aracnoiditis, esclerosis en placa, Pott vertebral, tumores, etc.) y a causas congénitas como el mielomeningocele (Figura 15).

\begin{tabular}{|c|c|}
\hline $\begin{array}{l}\text { Enfermedades congénitas y del } \\
\text { desarrollo }\end{array}$ & Parálisis cerebral, diastematomelia,... \\
\hline $\begin{array}{l}\text { Enfermedades/trastornos } \\
\text { degenerativos del SNC }\end{array}$ & $\begin{array}{l}\text { Esclerosis lateral amiotrófica (ELA), } \\
\text { paraparesia espástica hereditaria, } \\
\text { atrofia espinal muscular,... }\end{array}$ \\
\hline latrogenia & $\begin{array}{l}\text { Punciones medulares, colocación de } \\
\text { catéter epidural, reparación aórtica,... }\end{array}$ \\
\hline Infecciosas & $\begin{array}{l}\text { Viral: virus herpes simple, virus } \\
\text { varicela zóster, citomegalovirus, } \\
\text { HTLV-1, VIH, poliovirus,... } \\
\text { Bacteriana: enfermedad de Pott, } \\
\text { mycobacterium spp,... } \\
\text { Micosis: cryptococcus spp } \\
\text { Parasitaria: toxoplasma gondii, } \\
\text { Schistostoma mansoni,... }\end{array}$ \\
\hline Inflamatorias & $\begin{array}{l}\text { Esclerosis múltiple, mielitis } \\
\text { transversa... }\end{array}$ \\
\hline Neoplásicas & $\begin{array}{l}\text { Cáncer primario o metastásico } \\
\text { (intramedular y extramedular),... }\end{array}$ \\
\hline $\begin{array}{l}\text { Reumatológicas y } \\
\text { degenerativas }\end{array}$ & $\begin{array}{l}\text { Espondilosis, estenosis, patología } \\
\text { discal, enfermedad de Paget, artritis } \\
\text { reumatoide, osteoporosis, osificación } \\
\text { del ligamento longitudinal } \\
\text { posterior,... }\end{array}$ \\
\hline Secuela post-lesión & $\begin{array}{l}\text { Siringomielia, pérdida tardía de la } \\
\text { función... }\end{array}$ \\
\hline Tóxicas & Radiación, quimioterapia \\
\hline $\begin{array}{l}\text { Trastornos genéticos y } \\
\text { metabólicos }\end{array}$ & $\begin{array}{l}\text { Deficiencia de vitamina B12, } \\
\text { abetalipoproteinemia }\end{array}$ \\
\hline
\end{tabular}

Figura 15. Causas de lesión medular no traumática (tomado de ASPAYM, 2013). 


\section{INTRODUCCIÓN}

La mayor prevalencia de lesiones se puede encontrar entre la 3a y 4 a década de vida, aunque, debido al aumento de la esperanza de vida, existe una acentuación de lesión medular de origen médico en personas mayores de 65 años. Además, las lesiones medulares de origen traumático tienen mayor incidencia en los hombres que en las mujeres (con una relación de 4 a 1), y en cambio, en las lesiones con origen médico se igualan las cifras entre los sexos (ASPAYM, 2012).

El tipo de traumatismo y la anatomía humana, determinan la frecuencia de afectación de los diferentes niveles medulares y las consecuencias funcionales resultantes. La tetraplejia incompleta ocurre en un $31 \%$ de los lesionados, seguida de la paraplejia completa en un $26 \%$, la tetraplejia completa en un $22 \%$ y la paraplejia incompleta en un $19 \%$ de todos los casos (ASPAYM, 2012).

\subsubsection{Tipos de Lesión Medular y Clasificación}

La Organización Mundial de la Salud, en el documento "International perspectives on spinal cord injury" en 2013 (World Health Organization, 2013), determina las consecuencias de la lesión medular sobre las funciones motoras y sensoriales dependiendo de la severidad y nivel de la lesión. La clasificación neurológica y funcional de la lesión medular estandarizada internacionalmente se conoce como la Escala de Medición de la Discapacidad de la Asociación Americana de Lesión Medular (ASIA), o Escala ASIA, aprobada por la Sociedad Médica Internacional de Paraplejia en 1992, y revisada posteriormente en 1996, 2000 y 2006 (ASPAYM, 2013)(Figura 16). 


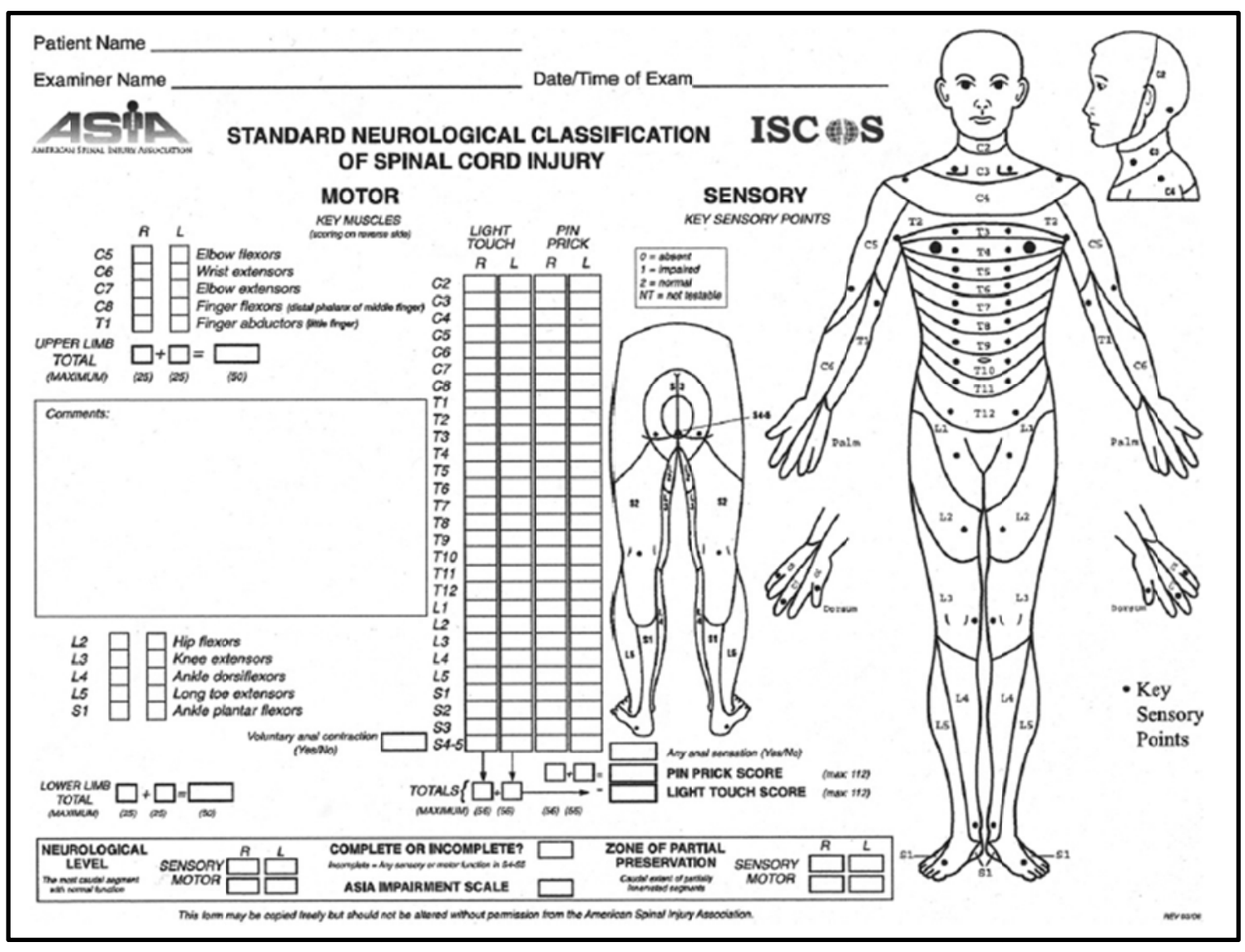

Figura 16. Escala de Medición de la Discapacidad de la Asociación Americana de Lesión Medular (ASIA) (tomado de American Spinal Injury Association).

Teniendo en cuenta la severidad de la lesión medular se pueden clasificar en dos tipos: lesión medular completa que implica pérdida total de sensibilidad y función motora por debajo del nivel de la lesión; y lesión medular incompleta en la que se mantiene parte de la función motora y sensorial por debajo del nivel de la lesión (Figura 17).

\begin{tabular}{|ll|}
\hline A & $\begin{array}{l}\text { Completa: no hay preservación de la función sensitiva o motora en } \\
\text { los segmentos sacros S4-S5. }\end{array}$ \\
\hline B & $\begin{array}{l}\text { Incompleta: preservación de la función sensitiva por debajo del } \\
\text { nivel neurológico que se extiende hasta los segmentos sacros S4-S5 } \\
\text { con ausencia de función motora. }\end{array}$ \\
\hline C & $\begin{array}{l}\text { Incompleta: preservación de la función motora por debajo del nivel } \\
\text { neurológico y más de la mitad de los músculos clave por debajo del } \\
\text { nivel neurológico tienen un grado menor a 3 (grado0-2). }\end{array}$ \\
\hline D & $\begin{array}{l}\text { Incompleta: preservación de la función motora por debajo del nivel } \\
\text { neurológico y al menos la mitad de los músculos clave por debajo } \\
\text { del nivel neurológico tienen un grado igual o mayor a 3. }\end{array}$ \\
\hline E & Normal: función sensitiva y motora normal. \\
\hline
\end{tabular}

Figura 17. Grado de afectación de la lesión medular según la escala ASIA (tomado de ASPAYM, 2013). 


\section{INTRODUCCIÓN}

El nivel de lesión de la médula espinal determinará las partes del cuerpo afectadas por parálisis sensorial y motora. La paraplejia se refiere a las lesiones de la medula espinal producidas a nivel torácico (T2-T12), a nivel lumbar (L1-L5) o a nivel sacro (S1-S5), lo que implica una variedad de falta de control de miembro inferior y tronco, sin afectación del miembro superior. El término tetraplejia se utiliza para describir lesiones a nivel cervical (C1-T1) que implicarán, dependiendo de la severidad de la lesión, una variedad de falta de control del cuello, tronco, miembro superior y miembro inferior (World Health Organization, 2013) (Figura 18).

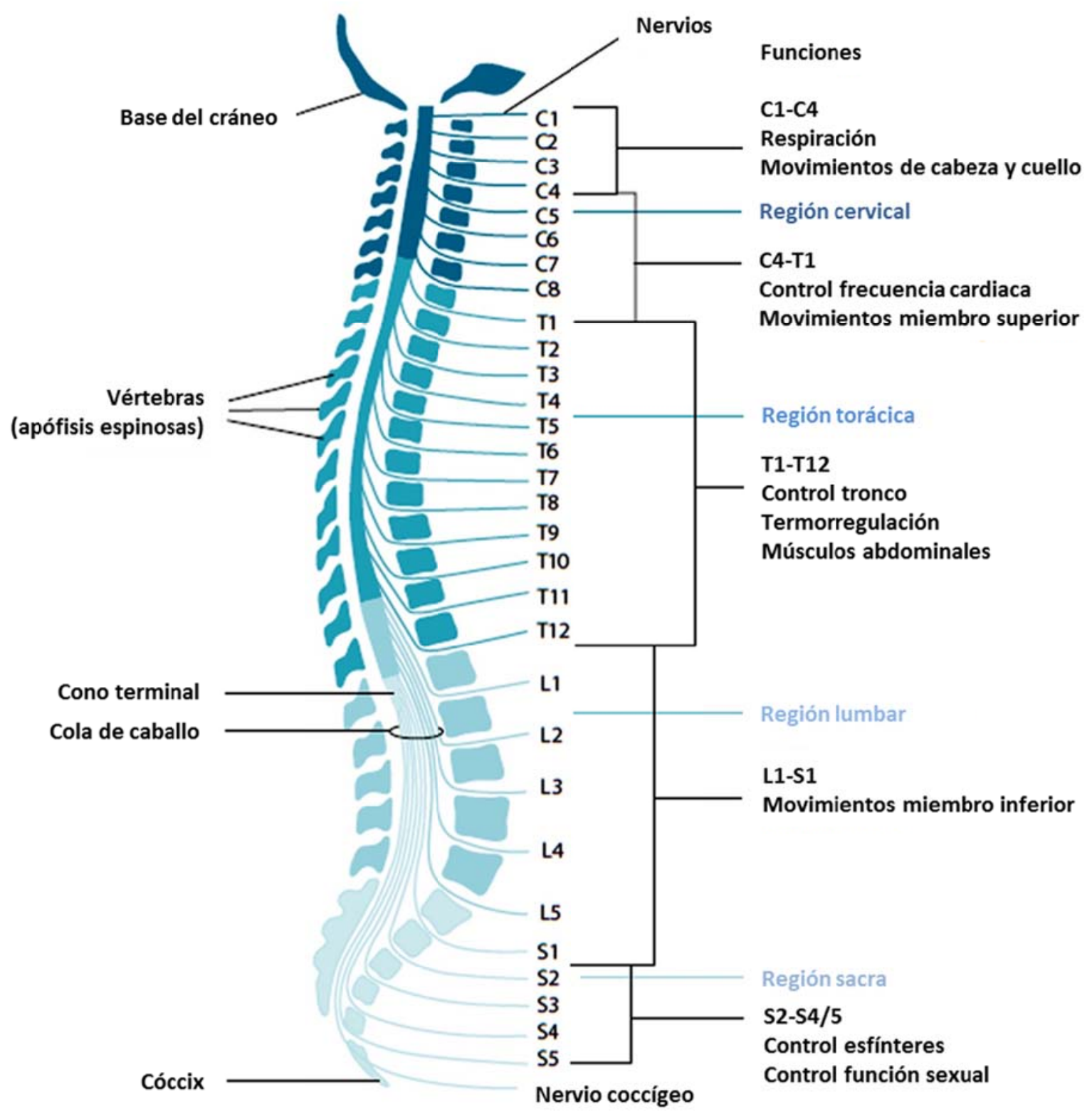

Figura 18. Organización longitudinal de la médula espinal y representación de sus funciones principales (adaptado de World Health Organization, 2013). 


\subsubsection{Consecuencias e Implicaciones de la Lesión Medular}

La consecuencia más obvia de la lesión medular es la pérdida de control voluntario de la musculatura estriada por debajo del nivel de la lesión por parálisis, que podrá presentarse en forma de debilidad muscular o alteración del tono muscular y reflejos dependiendo de la gravedad de la lesión. Otra consecuencia, ya mencionada, es la pérdida o alteración de la sensibilidad por debajo del nivel de la lesión, como la alteración o pérdida de la sensación al tacto, de la sensibilidad al dolor, de la sensibilidad para distinguir cambios de temperatura o de la propiocepción (ASPAYM, 2013; Levi, \& Hultling, 1999).

Además de la pérdida de la función motora y sensorial, la lesión medular afecta a las funciones neurológicas automáticas del cuerpo, como el control de esfínteres o la función sexual. Como consecuencia de la lesión medular y la interrupción de la comunicación entre el cerebro y los segmentos sacros (Figura 18), se presentan vejiga e intestinos neurógenos con pérdida de control voluntario de la micción y defecación. Es frecuente el uso del cateterismo como forma de control de esfínteres y del vaciado de la vejiga aun teniendo un alto impacto en el riesgo de infecciones del tracto urinario y siendo causa principal de reingreso en hospital y mortalidad prematura entre personas con lesión medular (World Health Organization, 2013). La función sexual también se encontrará alterada a causa de la interrupción de la comunicación entre cerebro (mediador de la respuesta sexual) y segmentos toracolumbares y sacros de la médula espinal (inervación de genitales) (Figura 18) (ASPAYM, 2013; Levi, \& Hultling, 1999)

A nivel del sistema cardiovascular se producen alteraciones como la hipotensión, arritmias cardíacas, disreflexia autónoma y trombosis venosa profunda. La hipotensión se manifiesta frecuentemente en paraplejia y tetraplejia como hipotensión ortostática con una disminución severa de la presión sanguínea a causa de un cambio de posición desde supino a bipedestación o sedestación, ocasionando mareos, visión borrosa, debilidad muscular e incluso pérdida de consciencia temporal. Las arritmias cardiacas ventriculares por disfunción del sistema nervioso autónomo o vegetativo y la bradicardia por mantenimiento de la inervación parasimpática se encuentran frecuentemente en personas con lesiones medulares en niveles altos (tetraplejia). La disreflexia autónoma consiste en una respuesta exagerada del sistema vegetativo desencadenada por una irritación vesical (sobredistensión, infección urinaria, espasmos vesicales y/presencia de cálculos) o intestinal (retención de heces fecales, distensión por gases o estímulos rectales), y que aparece con mayor frecuencia en lesiones en niveles iguales o superiores a T5. Se presenta con una vasoconstricción por debajo del nivel de lesión y una vasodilatación por encima de la lesión, lo que desencadena hipertensión arterial, cefalea pulsátil, piloerección, sudoración por encima del nivel de lesión, congestión nasal, bradicardia, ruborización, visión borrosa, inquietud, opresión en el pecho y dificultad para respirar. La 
trombosis venosa profunda se puede presentar, principalmente durante la fase aguda de la lesión, como consecuencia de la reducción de la circulación sanguínea en miembros inferiores. La causa de su aparición es la disfunción del sistema nervioso autónomo o vegetativo y la disminución del flujo local, mostrando un cuadro clínico de edema, piel tersa y brillante, dilatación venosa, aumento de la temperatura local, alteraciones de coloración y pulsos a nivel distal. La principal complicación de la trombosis venosa profunda es la tromboembolia pulmonar potencialmente letal y que se presenta con tos, fiebre, alteración del ritmo cardíaco, dificultad respiratoria y dolor u opresión torácica (ASPAYM, 2013; Levi, \& Hultling, 1999; World Health Organization, 2013).

Las complicaciones respiratorias, especialmente en lesiones altas, suponen la causa más común de fallecimiento tras la lesión medular. La debilidad y falta de coordinación entre el diafragma, músculos intercostales y musculatura abdominal alteran la función respiratoria, pérdida de capacidad inspiratoria y espiratoria, y limitan el reflejo de la tos, provocando hipoventilación pulmonar y colapso pulmonar por fatiga muscular y aumentando la acumulación de secreciones y el riesgo de infección e insuficiencia respiratoria (ASPAYM, 2013; World Health Organization, 2013).

El sistema neuromusculoesquelético, además de la pérdida de la función motora y sensorial, presenta otras alteraciones como la espasticidad muscular, la pérdida de densidad mineral ósea y la osificación heterotópica. La espasticidad, caracterizada por aumento de los reflejos tónicos y aumento de tono muscular, es una secuela importante de la lesión medular que se desarrolla progresivamente en los meses siguientes a la lesión, ocasionada por la continuación de las actividades automáticas de la médula espinal por debajo de la lesión que actúan sin regulación cerebral y de forma exaltada. Dependiendo de la intensidad de la espasticidad, sus efectos pueden ser incluso beneficiosos para la persona con lesión medular, como el mantenimiento de la masa muscular, la prevención parcial de la descalcificación ósea, la mejora de la circulación o la ayuda a la bipedestación y a las transferencias (ASPAYM, 2013). La pérdida de densidad mineral ósea se presenta principalmente en los primeros meses tras la lesión, en personas jóvenes, en pelvis y miembro inferior fundamentalmente. La mayor consecuencia de la osteoporosis es el riesgo de fractura ósea en miembros inferiores, diez veces más frecuente en lesiones completas que incompletas, y con mayor incidencia entre las personas con paraplejia que con tetraplejia, por su mayor movilidad y participación en actividades físicas. La osificación heterotópica se caracteriza por la formación anormal de tejido óseo en los tejidos blandos alrededor de las articulaciones por debajo del nivel de lesión lo que limita el rango de movimiento y la función articular, siendo más común en caderas, rodillas y hombros (ASPAYM, 2013; World Health Organization, 2013). 
Las alteraciones en la termorregulación, las alteraciones en la piel y el dolor son otras consecuencias derivadas de la lesión medular. Se produce una alteración del funcionamiento de sistema nervioso autónomo y somático, así como la comunicación entre el hipotálamo y la médula espinal, modificando las respuestas de vasodilatación o vasoconstricción, sudoración y escalofríos para la regulación de la temperatura (ASPAYM, 2013; Levi, \& Hultling, 1999).

La fragilidad de la piel (debido a alteraciones vasculares y nerviosas) sumada a la alteración o pérdida de la capacidad para la movilidad, las alteraciones de la sensibilidad y el tiempo prolongado en una misma posición tienen como consecuencia el aumento del riesgo a presentar una úlcera por presión (escaras) en la piel. El daño producido en la piel, generalmente en zonas vulnerables como prominencias óseas, puede ir desde el eritema epidérmico hasta la destrucción y necrosis de piel, fascia o músculo alcanzando el plano óseo. Además, la mayoría de personas con lesión medular refieren dolor neuropático crónico por debajo del nivel de lesión en forma de quemazón, dolor punzante o dolor nervioso, lo que tiene un impacto significativo en su calidad de vida (ASPAYM, 2013; Levi, \& Hultling, 1999; World Health Organization, 2013).

\subsubsection{Necesidades de Lesionados Medulares: Importancia de la Bipedestación}

La recuperación de la capacidad de caminar fue identificada como el objetivo más importante por pacientes con lesión medular, independientemente del nivel de lesión, tiempo de evolución desde la lesión o la edad del paciente, por delante de otras funciones como comer, vestirse, asearse, controlar esfínteres, realizar transferencias a la cama o ducha, superar escaleras, dominar la silla de ruedas o ir al baño (Ditunno, Patrick, Stineman, \& Ditunno, 2008; Ditunno, \& Scivoletto, 2009). Además, la falta de capacidad para caminar o moverse se identifica también entre los pacientes como la tarea más complicada de asimilar y que implica mayor limitación tras la lesión por delante del control de esfínteres, la función sexual o la aparición del dolor (Widerström-Noga, Felipe-Cuervo, Broton, Duncan, \& Yezierski, 1999), y el autocuidado y las tareas domésticas (ASPAYM, 2012).

Numerosos autores señalan los beneficios de la bipedestación y el caminar como parte de los programas de rehabilitación en pacientes con lesión medular: reducción del tono muscular y espasticidad e incremento de rango articular y longitud del músculo (Adams, \& Hicks, 2011; Ben et al., 2005; Shields, \& Dudley-Javorski, 2005), mejora en la eficiencia y regularidad de la función de sistema urinario y digestivo (Shields, \& Dudley-Javorski, 2005), reducción significativa en la desmineralización ósea con la bipedestación temprana (Alekna, Tamulaitiene, Sinevicius, \& Juocevicius, 2008; Ben et al., 2005; Dionyssiotis, Lyritus, Mavrogenis, \& Papagelopoulos, 2011), mejora de la función cardiovascular y respiratoria (Eng, 


\section{INTRODUCCIÓN}

Levins, Townson, Mah-Jones, Bremner, \& Huston, 2001), reducción o prevención de la hipotensión ortostática, mejora del estado de la piel y reducción de las escaras por presión (Eng et al., 2001), mejora en el control postural, incremento de fuerza en músculos posturales, mejora del equilibrio y mantenimiento de los rangos y los patrones de movimiento durante la marcha. Se presentan también beneficios percibidos por los pacientes en la mejora de la calidad de vida relacionada con la bipedestación y la mejora de la condición física, la mejora del sueño y la mejora de la capacidad para el autocuidado (Eng et al., 2001). Además, se perciben mejoras subjetivas en la circulación, la función del sistema urinario y digestivo, la respiración, el estado de la piel, el sueño y la reducción del dolor, espasmos y tumefacción (Eng et al., 2001). 


\subsection{Robots}

\subsubsection{Dispositivos para la Rehabilitación y Compensación de la Marcha}

El término exoesqueleto originalmente se ha referido a dispositivos mecánicos con estructura antropomórfica que eran llevados por un usuario con el objetivo de aumentar su rendimiento, puesto que las primeras investigaciones científicas y tecnológicas sobre exoesqueletos comenzaron en el ámbito militar con el objetivo de aumentar las capacidades de sujetos sanos. Posteriormente el término exosqueleto se ha comenzado también a utilizar para describir dispositivos para la rehabilitación y asistencia de la movilidad de personas con patologías neuromusculoesqueléticas, concretamente si implican la totalidad del miembro inferior.

En los años 60 con el impulso de programas militares en Estados Unidos (Defense Advanced Research Projects Agency (DARPA)), se comenzaron a desarrollar exoesqueletos para aumentar el rendimiento de los soldados en el transporte de cargas (Exoskeletons for Human Performance Augmentation (EHPA)), pudiendo incrementar el tamaño de la carga y reduciendo la fatiga durante la realización de la tarea (Dollar, \& Herr, 2008). Algunos ejemplos de este tipo de exoesqueletos son el BLEEX (Berkeley Lower Extremity Exoskeleton), Sarcos Exoskeleton Wearable Energetically Autonomus Robot (WEAR), MIT Exoskeleton (HarvardMassachusetts Institute of Technology Exoskeleton), y posteriormente HAL-5 (Hybrid Assistive Limb), NTU-LEE (NTU Lower Extremity Exoskeleton) y Nurse-Assisting Exoskeleton (Dollar, \& Herr, 2008; Herr, 2009; Low, 2011) (Figura 19).
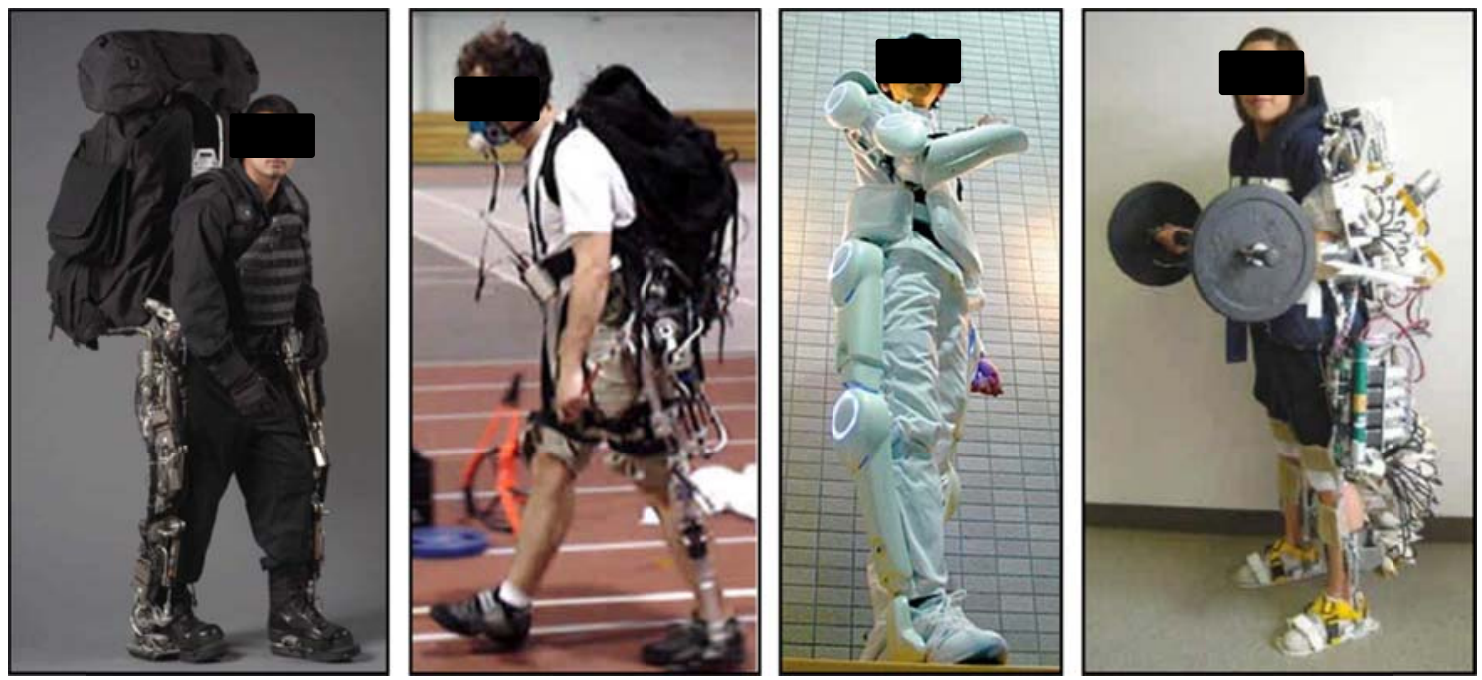

Figura 19. Exoesqueletos para el incremento de fuerza en sujetos sanos. De izquierda a derecha: BLEEX, MIT Exoskeleton, HAL-5 y Nurse-Assisting Exoskeleton (tomado de Dollar, \& Herr, 2008).

Este apartado en concreto, se centra en la descripción de los dispositivos desarrollados para la rehabilitación y compensación de la marcha en los últimos años, no se detallan otro tipo de 


\section{INTRODUCCIÓN}

dispositivos como las órtesis pasivas (Del-Ama, Koutsou, Moreno, de-los-Reyes, Gil-Agudo, \& Pons, 2012). Los exoesqueletos para la rehabilitación y compensación de la marcha se originaron como medio de mejora de los tiempos y calidad de las sesiones de tratamiento. Las sesiones de rehabilitación de la marcha involucran entre dos y tres terapeutas trabajando de forma simultánea para guiar el movimiento de miembro inferior, pelvis y tronco de los pacientes durante la marcha. Este tipo de sesiones implican una gran demanda física por parte del terapeuta, lo que no garantiza la calidad del movimiento durante la sesión y el tiempo suficiente para un entrenamiento óptimo (Esclarín-de Ruz, Alcobendas-Maestro, CasadoLópez, Muñoz-González, Florido-Sánchez, \& González-Valdizán, 2009; Swinnen, Duerinck, Baeyens, Meeusen, \& Kerckhofs, 2010; Wirz, Bastiaenen, de Brie, \& Dietz, 2011). La incorporación de sistemas electromecánicos en las sesiones de rehabilitación contribuye en una mejora de los patrones biomecánicos durante la sesión de entrenamiento, mejora de los estímulos propioceptivos, menor fatiga de los terapeutas y sesiones con mayor duración. Ante la amplia variedad de dispositivos desarrollados para la rehabilitación y compensación de la marcha se clasifican una selección relevante de éstos dentro de dos grandes grupos: Exoesqueletos combinados con tapiz rodante y Exoesqueletos con desplazamiento sobre el suelo (Chen, Chan, Guo, \& Yu, 2013; Low, 2011).

\subsubsection{Exoesqueletos combinados con tapiz rodante}

Los exoesqueletos combinados con tapiz rodante se componen de una órtesis motorizada de miembro inferior, un sistema de suspensión/soporte de peso y un tapiz rodante. La órtesis motorizada reproduce la cinemática de cadera, rodilla y tobillo y, en algunos prototipos, también la cinemática de la pelvis; por otro lado, el sistema de suspensión/soporte de peso controla la posición erguida del tronco y proporciona estabilidad durante la marcha. Este tipo de dispositivos está ampliamente distribuido por hospitales y centros de rehabilitación en la actualidad. El más conocido y comercializado es Lokomat (Colombo, Joerg, Schreier, \& Dietz, 2000), y similar a este se pueden encontrar otros como ReoAmbulator, ALEX (Active Leg Exoskeleton) y LOPES (LOwer-extremity Powered Exoskeleton) (Chen et al., 2013; Low, 2011) (Figura 20). 


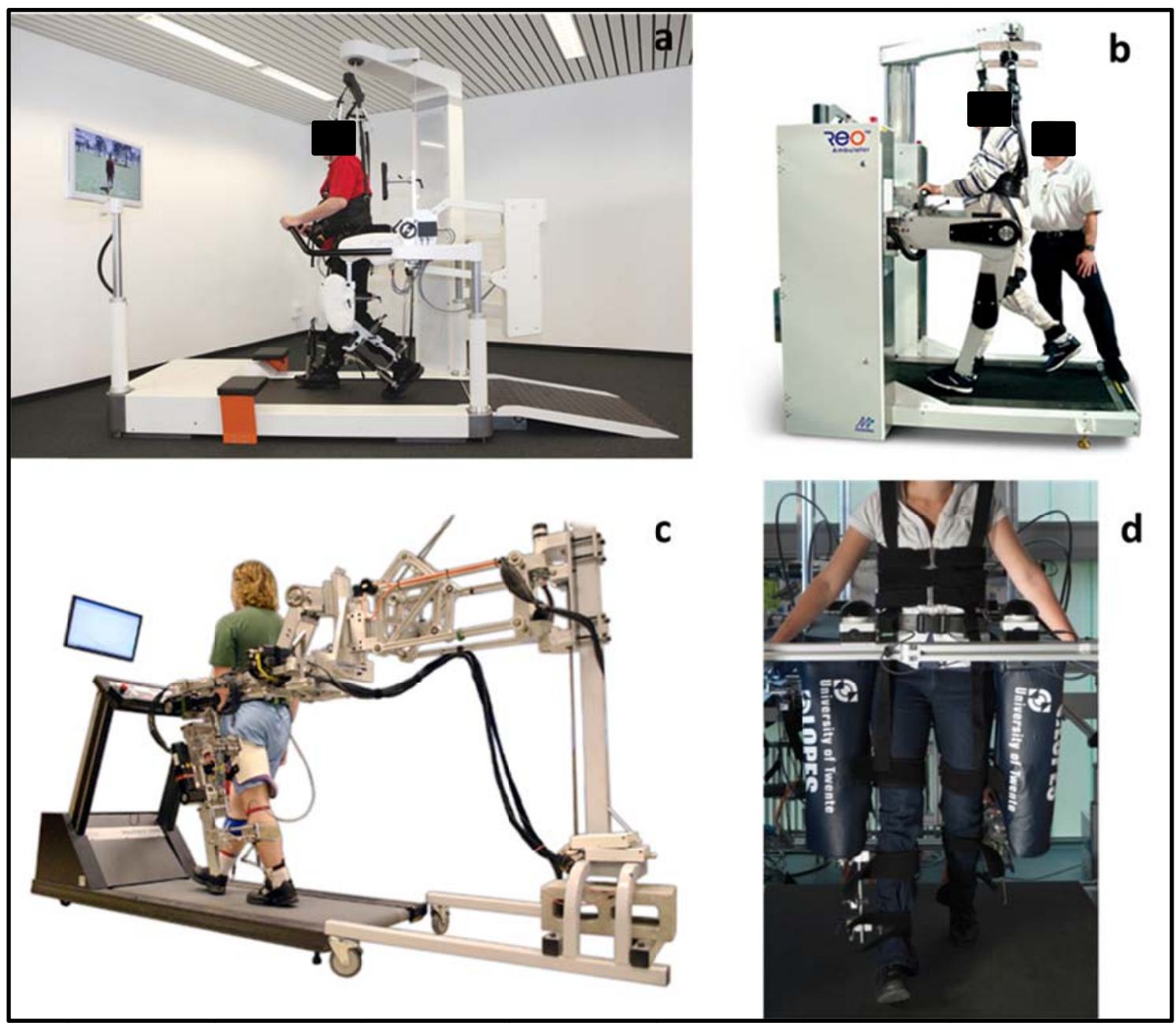

Figura 20. Exoesqueletos combinados con tapiz rodante: (a) Lokomat (tomado de Medical Expo, 2015),

(b) ReoAmbulator (tomado de Medical Expo, 2015), (c) ALEX (tomado de Robotics and Rehabilitation Laboratory, 2015) y (d) LOPES (tomado de Donati et al., 2013).

\subsubsection{Exoesqueletos con desplazamiento sobre el suelo}

En la actualidad, existen dos tipos de exoesqueletos con desplazamiento sobre el suelo para miembro inferior. El primer grupo se caracteriza por ser una órtesis motorizada de miembro inferior, con control únicamente de cadera y rodilla. La ventaja fundamental que ofrecen es el procurar un entorno más natural para la rehabilitación y con menor impacto visual, aunque la gran mayoría de dispositivos necesitan apoyos externos, como muletas o andadores, para facilitar el equilibrio y la transferencia de peso. Este tipo de dispositivos se están comercializando o comienzan a comercializarse en la actualidad con el objetivo de complementar y completar la rehabilitación en el domicilio del paciente, y como forma de compensación o sustitución de la marcha durante la vida diaria en su propio domicilio o trabajo. Además, también se están introduciendo en hospitales y centros de rehabilitación como alternativa a los exoesqueletos combinados con tapiz rodante (Chen et al., 2013). ReWalk, Ekso Bionics y Rex Bionics (Figura 21) son ejemplos de empresas internacionales que 


\section{INTRODUCCIÓN}

están desarrollando este tipo de exoesqueletos aunque, actualmente, empresas nacionales como Technaid y Marsi Bionics, creadas a partir de proyectos desarrollados en el Centro de Automática y Robótica del Consejo Superior de Investigaciones Científicass, también están desarrollando este tipo de dispositivos para rehabilitación de pacientes que han sufrido accidentes cerebrovasculares (H2 - Technaid) y para niños con afecciones en el sistema neuromusculoesquelético (Atlas 2020 - Marsi Bionics) (Figura 22).
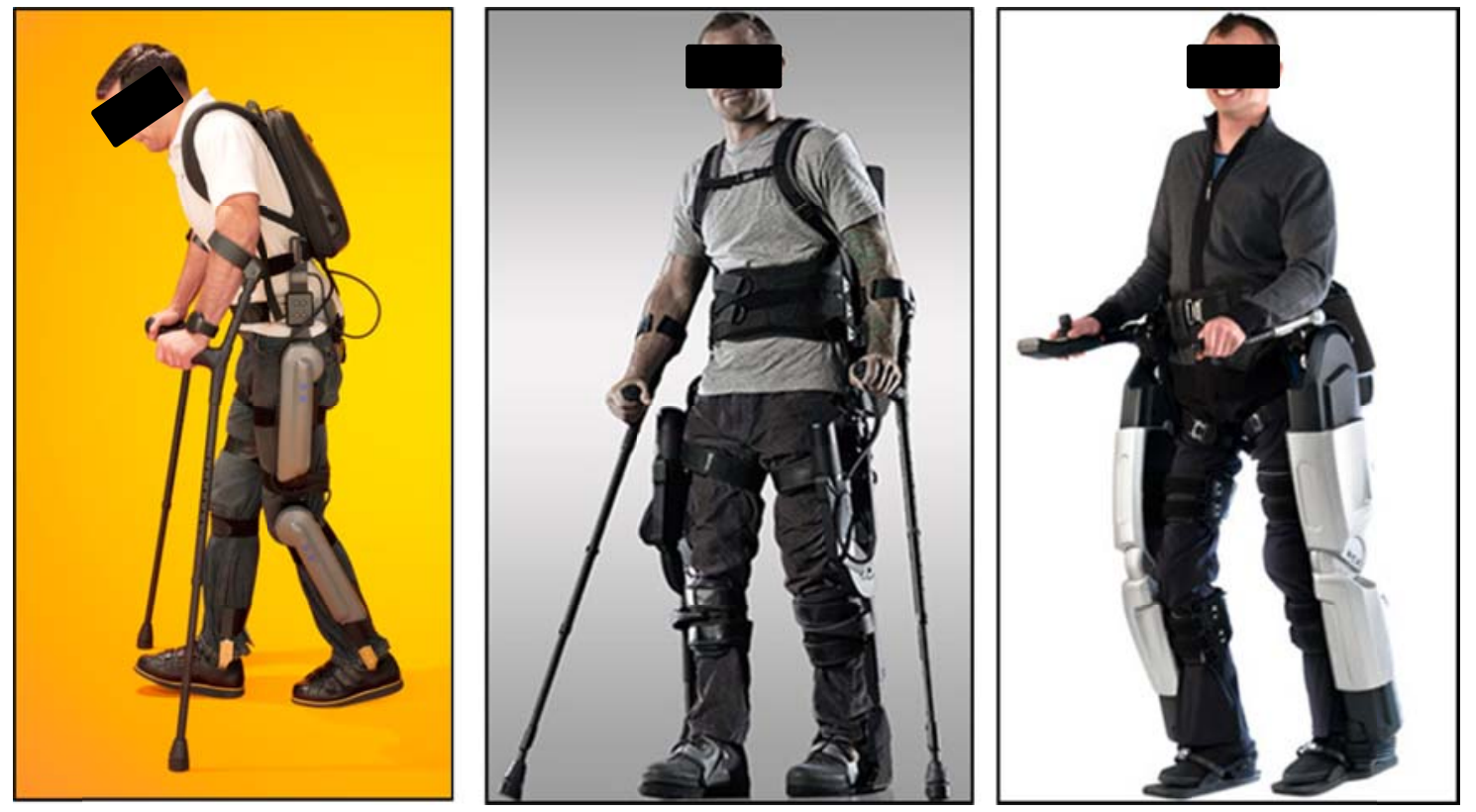

Figura 21. Exoesqueletos internacionales con desplazamiento sobre el suelo. De izquierda a derecha: ReWalk Personal 6.0 System (tomado de ReWalk, 2015), Ekso (tomado de Ekso Bionics, 2015) y Rex (tomado de Rex Bionics, 2015). 

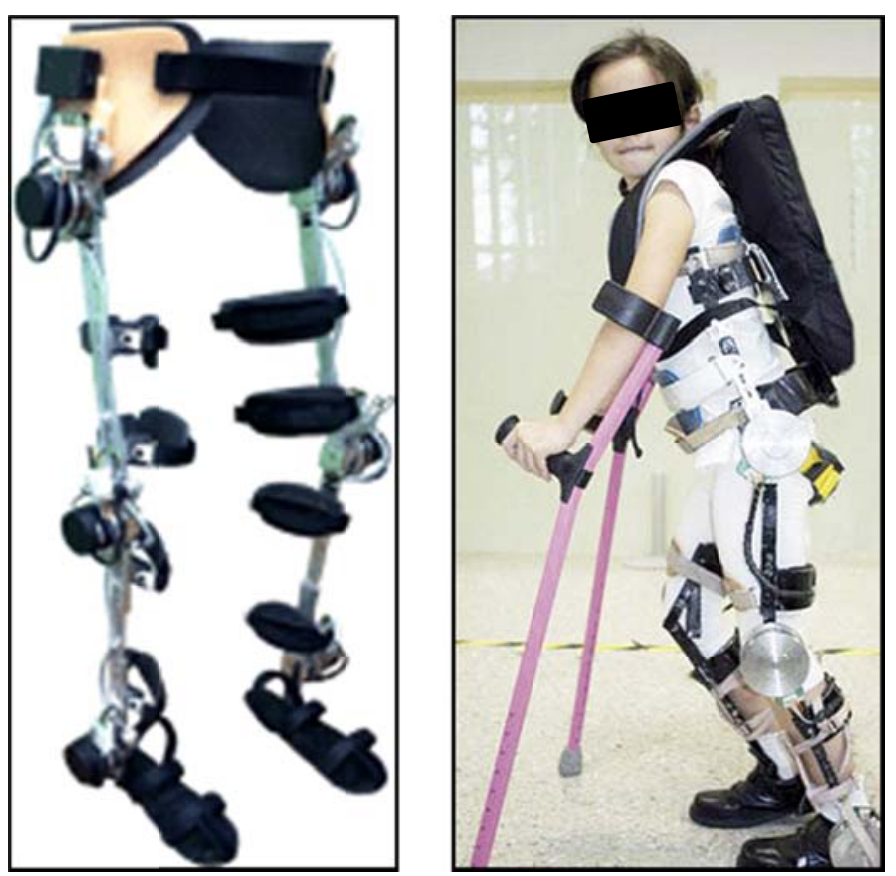

Figura 22. Exoesqueletos nacionales con desplazamiento sobre el suelo. De izquierda a derecha: $\mathrm{H} 2$ Exoesqueleto (tomado de Technaid, 2015) y Atlas 2020 (tomado de Marsi Bionics, 2015).

El segundo tipo de exoesqueletos con desplazamiento sobre el suelo se utilizaría únicamente en el medio hospitalario o de centro de rehabilitación. No son dispositivos que puedan utilizarse en el domicilio del paciente, pero tienen la ventaja fundamental de incluir andadores motorizados que permiten la descarga parcial de peso, mantienen una posición erguida del tronco, y controlan la estabilidad y equilibrio durante la marcha. Dichos aspectos facilitan el uso por parte de pacientes de mayor gravedad o con menor control de losi movimientos del tronco. Además, siguen ofreciendo un entorno más natural para la rehabilitación y con menor impacto visual que los exoesqueletos combinados con tapiz rodante. Este tipo de dispositivos estarían compuestos por una órtesis motorizada con control de cadera, rodilla, tobillo y pelvis, y un andador motorizado con sistema de descarga parcial de peso a través de un arnés. El ejemplo más conocido y comercializado de los exoesqueletos con desplazamiento sobre el suelo es el dispositivo WalkTrainer (Figura 23), aunque otros prototipos comienzan a desarrollarse como NaTUre-gait, o el dispositivo desarrollado en el presente estudio (HYBRID). 

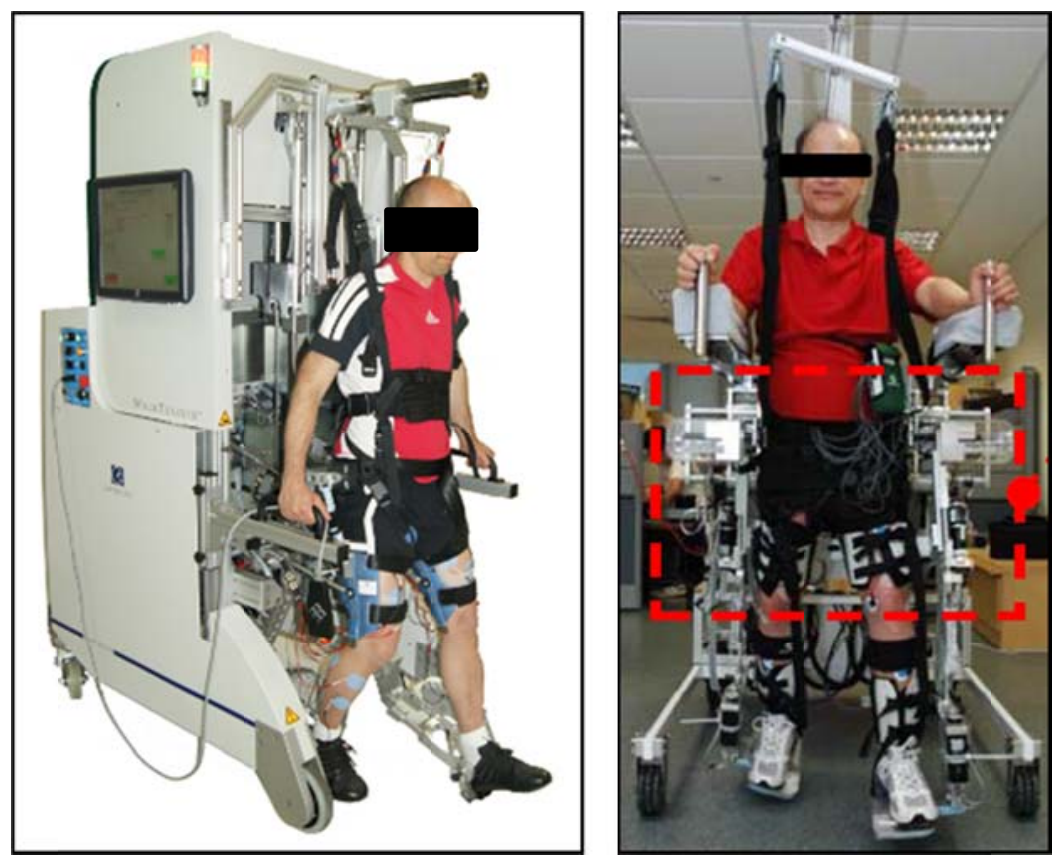

Figura 23. Exosqueletos con desplazamiento sobre suelo y sistema de descarga de peso. De izquierda a derecha: WalkTrainer (tomado de Swortec, 2015) y NaTUre-gait (tomado de Luu, Low, Qu, Lim, \& Hoon, 2014).

\subsubsection{Descripción del sistema HYBRID y su Funcionamiento}

El sistema HYBRID, desarrollado por el Consejo Superior de Investigaciones Científicas (CSIC) en el contexto del Proyecto I+D+i Hybrid Technological Platform for Rehabilitation, Functional Compensation and Training of Gait in Spinal Cord Injury (SCI) Patients (DPI2011-28160-C03-02), trata de estudiar, definir e implementar un nuevo sistema de rehabilitación y compensación de la marcha para personas con lesión medular. Para ello, se combina el uso de un exoesqueleto con sistemas externos de apoyo o de estabilización activa de la marcha, con el objetivo de recuperar capacidades funcionales induciendo la ejecución de patrones de la marcha a través de los movimientos de las articulaciones del miembro inferior.

El exoesqueleto está compuesto por una órtesis activa derecha y otra izquierda unidas en su parte superior por una cesta pélvica, y que incluyen tres motores correspondientes a las articulaciones del miembro inferior (cadera, rodilla y tobillo). Los motores están diseñados para el movimiento en el plano sagital (movimientos de flexo-extensión), no permitiendo el movimiento en el plano frontal y transversal de la cadera, la rodilla, el tobillo y la pelvis (Figura 24).

El andador motorizado (REMOVI) permite abordar, controlando la bipedestación, el equilibrio y la locomoción, un reentrenamiento de la marcha con mayor grado de libertad que en un tapiz rodante. Asiste, mediante un desplazamiento real del sujeto, el trabajo del control de giros, aceleraciones, deceleraciones, comportamiento ante superficies irregulares, obstáculos 
y superficies inclinadas. La estructura REMOVI está compuesta por una amplia base, con dos ruedas delanteras motorizadas y dos traseras libres, y una estructura central con dos brazos de elevación que realizan la transferencia del usuario de la silla de ruedas a la posición de bipedestación, y soportan parte del peso y estabilizan al usuario mediante un arnés durante la marcha (Figura 24 y Figura 25).
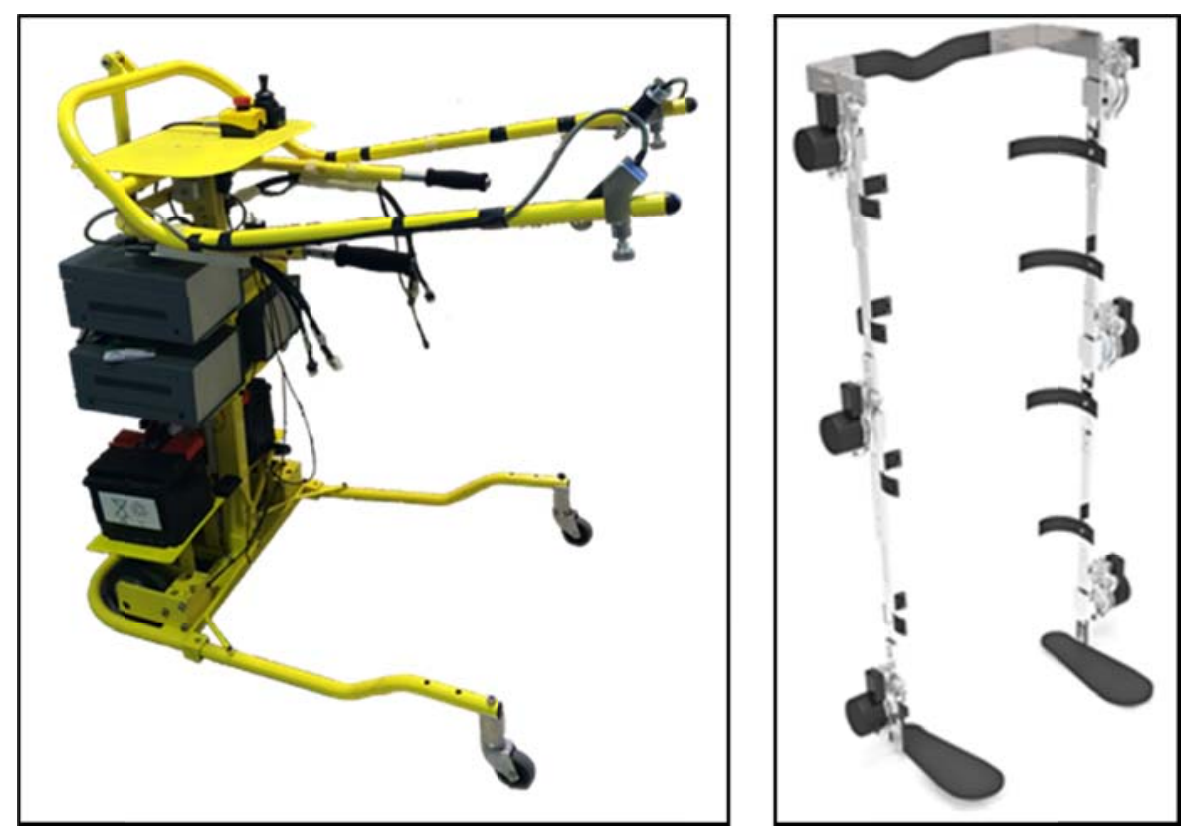

Figura 24. Dispositivo HYBRID. De izquierda a derecha: Andador motorizado con sistema de descarga de peso y órtesis activa con control de cadera, rodilla y tobillo (tomado de Urendes, Asín, Gómez, Ceres, \& Pons, 2014).

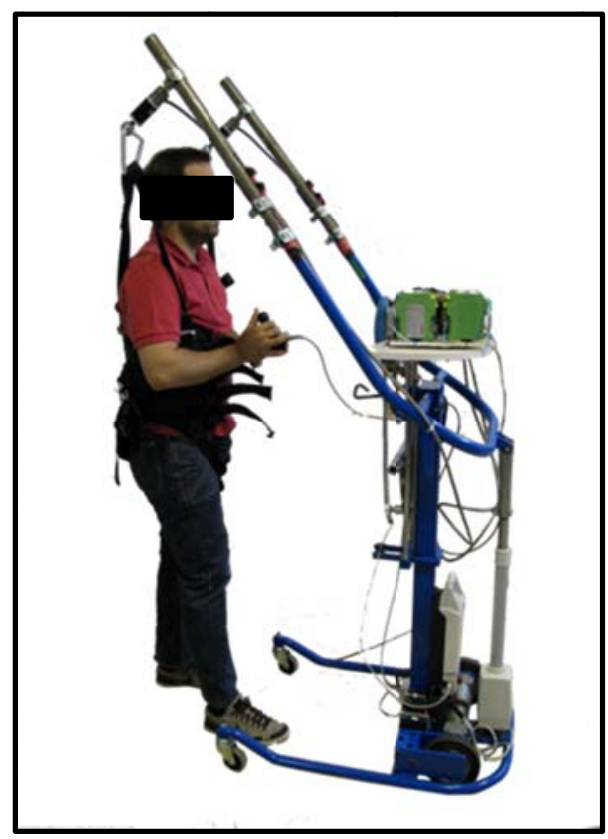

Figura 25. Estructura REMOVI (tomado de Urendes et al., 2014) 
Estos dos dispositivos, independientes, funcionan de manera sincronizada gracias a la interacción entre el usuario y el sistema REMOVI, que se basa en el seguimiento de la plataforma a los movimientos del sujeto. El exoesqueleto, por tanto, toma la función de guía, mientras que el andador motorizado deberá seguir sus movimientos, manteniendo una distancia constante entre ambos dispositivos. La combinación de ambos, exoesqueleto y andador motorizado, mejora la seguridad, la adaptación y el consumo de energía de los sujetos que lo utilizan durante la rehabilitación y compensación de la marcha (Figura 26).

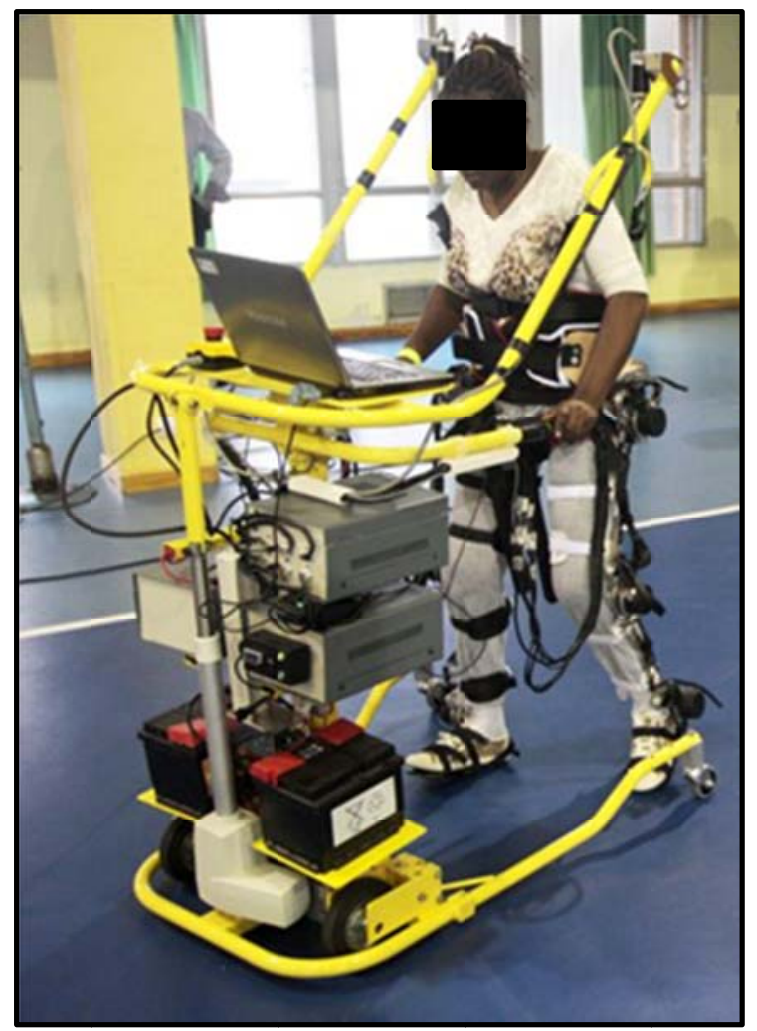

Figura 26. Usuaria del dispositivo HYBRID en el Hospital de Parapléjicos de Toledo (tomado de Bow, 2014).

\subsubsection{Rehabilitación con Exoesqueletos en pacientes con Lesión Medular}

Las terapias físicas, en sus diferentes tipos, forman parte de la rutina de los programas de rehabilitación y mantenimiento de las personas con lesión medular. Estas intervenciones incluyen entrenamientos de fuerza, resistencia, reeducación de la marcha, estiramientos y terapia manual siendo efectivas en la mejora de la debilidad muscular, la resistencia cardiovascular, la movilidad articular, la flexibilidad muscular, la pérdida de masa ósea, el dolor o la espasticidad (Harvey, Lin, Glinsky, \& De Wolf, 2009).

La reeducación de la marcha con dispositivos mecánicos se presenta como alternativa a la reeducación de la marcha manual guiada por terapeutas, mencionada con anterioridad, para la 
mejora de las condiciones de las sesiones de tratamiento. Siendo un método de tratamiento que en la actualidad está desarrollándose, siguen estudiándose sus beneficios en las funciones corporales y la transferencia a las actividades diarias. Diferentes estudios describen programas de tratamiento con exoesqueletos, fundamentalmente sobre tapiz rodante y con descarga de peso corporal, como Lokomat (Esclarín-De Ruz et al., 2009; Fritz et al., 2011; Swinnen et al., 2010; Wirz et al., 2011). Los programas descritos, siendo muy heterogéneos, establecen sesiones de reeducación de la marcha de entre 30 y 60 minutos al día (en ningún caso se describen sesiones de menos de 20 minutos), entre 3 y 5 veces por semana y alrededor de 8 semanas de media (6-12 semanas).

Los principales beneficios físicos de este método de tratamiento para la reeducación de la marcha se reflejan fundamentalmente en aumento de la fuerza en miembro inferior (Motricity Index leg score y Lower Extremity Motor score) y velocidad de la marcha (10 Meter Walk Test (10MWT)), aumento de la resistencia (6 Minutes Walk Test (6MWT)), aumento de la movilidad (Timed Go Walk test y Timed Up and Go test), aumento del equilibrio (Berg Balance scale), y disminución de la espasticidad y frecuencia de aparición de espasmos musculares (Spinal Cord Assessment Tool for Spasticity, Asworth scale y Penn Spasm Frecuency scale). Además, también se aumenta la independencia funcional de los usuarios y se reduce la necesidad de ayudas técnicas para la deambulación (Functional Independence Measure (FIM-L) y Walking Index for Spinal Cord Injury-II (WISCI-II)) (Esclarín-De Ruz et al., 2009; Fritz et al., 2011; Swinnen et al., 2010; Wirz et al., 2011). 



\section{Planteamiento del problema, Objetivos e Hipótesis}





\subsection{Planteamiento del problema}

El sistema HYBRID, como otros dispositivos mencionados, trata de implementar un sistema de rehabilitación y compensación de la marcha para personas con lesión medular. La mayoría de estos exoesqueletos ya se están utilizando en el ámbito hospitalario en las terapias físicas y programas de recuperación tras la lesión medular, como alternativa a la reeducación de la marcha manual guiada por terapeutas.

Aunque todos los dispositivos robóticos tienen el movimiento humano como estándar de referencia, no se evalúa de manera habitual el movimiento que los exoesqueletos reproducen, y su similitud o diferencias con el patrón de la marcha humana, o las modificaciones y adaptaciones que sufren los patrones teóricos al interaccionar con el cuerpo del paciente.

En el presente estudio se trata de analizar cuáles son las características de la marcha normal, y que diferencias se encuentran entre la marcha normal y el patrón de marcha lenta que reproduciría teóricamente el exoesqueleto. Finalmente, se pretende evaluar que modificaciones y adaptaciones sufre el patrón de marcha lenta teórico al reproducirlo por exoesqueleto a diferentes porcentajes de descarga de peso, e interaccionando con las fuerzas y resistencias que ofrece el cuerpo de cada sujeto. Todo ello, serviría para determinar la idoneidad o no del sistema HYBRID para reproducir patrones dirigidos a la rehabilitación o compensación de la marcha. Para ello, se tomará como referencia la apariencia natural de la marcha reproducida, el movimiento descrito por el centro de gravedad, la cinemática de las articulaciones del miembro inferior y la coordinación existente entre ellas (Torricelli et al., 2015). 
OBJETIVOS

\subsection{Objetivos de la Investigación}

Para evaluar el dispositivo HYBRID y determinar la idoneidad del patrón reproducido por el exoesqueleto para la rehabilitación o compensación de la marcha se establecen una serie de objetivos en las diferentes etapas del estudio:

1. Examinar las diferencias en la marcha a velocidad normal en función del sexo y en función de la estatura de los sujetos.

2. Comparar los patrones de marcha a velocidad lenta $(0,25 \mathrm{~m} / \mathrm{s})$ con los patrones a velocidad normal.

3. Comparar los patrones de la marcha reproducidos por el exoesqueleto con el $30 \%$, $50 \%$ y $70 \%$ de descarga del peso corporal con los patrones a velocidad lenta $(0,25 \mathrm{~m} / \mathrm{s})$. 
HIPÓTESIS

\subsection{Hipótesis de la Investigación}

El presente trabajo de investigación parte de la formulación de las siguientes hipótesis:

1. Existen diferentes patrones de la marcha a velocidad normal en función del sexo y la estatura de los sujetos.

2. El patrón de marcha a velocidad lenta se muestra diferente al patrón a velocidad normal.

3. La descarga de peso corporal con el dispositivo HYBRID y la interacción con el cuerpo del sujeto modifican el patrón de marcha lenta implementado en el dispositivo. 

3. Metodología 



\subsection{Diseño de Investigación}

La presente investigación consiste en un estudio cuantitativo transversal, realizado en el contexto del Proyecto I+D+i Hybrid Technological Platform for Rehabilitation, Functional Compensation and Training of Gait in Spinal Cord Injury (SCl) Patients, que la Universidad Politécnica de Madrid ha desarrollado junto al Consejo Superior de Investigaciones Científicas y el Hospital de Parapléjicos de Toledo (DPI2011-28160-C03-02) desde enero de 2011 hasta diciembre de 2014.

La tesis se ha efectuado de forma simultánea a las tareas del proyecto I+D+i planteadas para la Facultad de Ciencias de la Actividad Física y el Deporte, de estudio y análisis de patrones de la marcha para la implementación en el software del dispositivo Hybrid y su posterior reproducción con sujetos sanos (Tabla 1 ).

Tabla 1. Cronograma de las tareas del proyecto realizadas durante la investigación.

\begin{tabular}{|c|c|c|c|c|c|c|c|c|c|c|c|c|}
\hline Año/Mes & 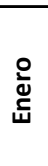 & 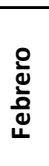 & $\begin{array}{l}\frac{0}{N} \\
\frac{N}{\pi}\end{array}$ & $\begin{array}{l}\bar{g} \\
\frac{8}{\alpha}\end{array}$ & $\begin{array}{l}\stackrel{0}{\frac{1}{\pi}} \\
\sum^{\frac{\pi}{2}}\end{array}$ & 옫 & $\stackrel{\circ}{\frac{0}{3}}$ & $\begin{array}{l}\stackrel{8}{\tilde{H}} \\
\text { 品 }\end{array}$ & 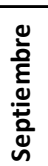 & 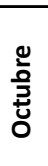 & 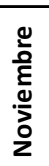 & 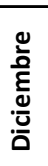 \\
\hline 2011 & & & & & & & & & & & & \\
\hline 2012 & & & & & & & & & & & & \\
\hline 2013 & & & & & & & & & & & & \\
\hline 2014 & & & & & & & & & & & & \\
\hline 2015 & & & & & & & & & & & & \\
\hline
\end{tabular}

Formación en Estudio de la Marcha y Revisión de Protocolos de Medida. Diseño del Plan de Investigación.

Ensayo de Protocolos para Estudio 1. Obtención y clasificación de la Muestra.

Estudio 1: Toma de Datos de Marcha Normal y Lenta con Sujetos Sanos (Objetivo 1 y 2).

Procesamiento de Datos de Estudio 1. Determinación de los patrones de la marcha a velocidad normal y lenta.

Ensayo de Protocolos para Estudio 2.

Estudio 2: Toma de Datos de Marcha con HYBRID con Sujetos Sanos (Objetivo 3).

Procesamiento de datos de Estudio 2.

Análisis Estadístico de los Datos y Redacción de la Tesis 
METOdOLOGÍA

\subsection{Muestra}

\subsubsection{Muestra sujetos sanos y distribución en grupos por estatura (Estudio 1)}

Para establecer los patrones normales de la marcha se seleccionaron y analizaron a 62 sujetos, 29 mujeres $(26,10 \pm 8,18$ años; $55,88 \pm 4,97 \mathrm{~kg}$ y $165,30 \pm 4,96 \mathrm{~cm})$ y 33 hombres $(24,00 \pm 4,37$ años; $72,15 \pm 6,84 \mathrm{~kg}$ y $177,42 \pm 6,07 \mathrm{~cm}$ ) distribuidos en grupos considerando los percentiles 25, 50 y 75 de estatura de la población española (Tabla 2). El primer grupo (H1 y M1) corresponde a sujetos situados por debajo del percentil $25(<172,9$ para hombres y $<159,8$ para mujeres), el segundo grupo (H2 y M2) se encuentra entre los percentiles 25 y 50 (172,9177 para hombres y 159,8-163,8 para mujeres), el tercer grupo (H3 y M3) lo componen sujetos que se encuentran entre los percentiles 50 y 75 (177-181,4 para hombres y 163,8-167,9 para mujeres) y el cuarto grupo (H4 y M4) sujetos ubicados por encima del percentil 75 (>181,4 para hombres y $>167,9$ para mujeres) de la estatura de la población española (Carrascosa et al., 2008) (Figura 27). Todos los sujetos participaron de forma voluntaria y firmaron el consentimiento informado para la realización de las pruebas. Todos los protocolos aplicados fueron previamente aprobados por el Comité de Ética de la Universidad Politécnica de Madrid. Se establecieron como criterios de inclusión el no padecer patologías crónicas del sistema musculo-esquelético, no haber sufrido lesiones recientemente y tener un índice de masa corporal entre 18,5 y 24,99 establecido como el rango de normopeso por la Organización Mundial de la Salud.

Tabla 2. Características antropométricas de la muestra

\begin{tabular}{lccccc}
\hline & \multicolumn{5}{c}{ Hombres (Media \pm DS) } \\
\cline { 2 - 6 } Número de Sujetos & H1 & H2 & H3 & H4 & Total \\
\cline { 2 - 6 } Edad (años) & 9 & 7 & 7 & 10 & 33 \\
Peso (kg) & $26,00 \pm 5,45$ & $23,29 \pm 5,88$ & $23,86 \pm 2,27$ & $22,80 \pm 2,97$ & $24,00 \pm 4,37$ \\
Estatura (cm) & $66,76 \pm 3,55$ & $72,39 \pm 5,31$ & $73,07 \pm 6,65$ & $76,21 \pm 7,67$ & $72,15 \pm 6,84$ \\
Longitud de Pierna (cm) & $169,86 \pm 1,71$ & $175,26 \pm 1,48$ & $179,29 \pm 0,95$ & $184,45 \pm 2,80$ & $177,42 \pm 6,07$ \\
Anchura de Pelvis (cm) & $27,13 \pm 3,04$ & $90,68 \pm 1,78$ & $92,54 \pm 3,00$ & $96,53 \pm 4,22$ & $91,88 \pm 4,81$ \\
& $22,89 \pm 2,23$ & $23,86 \pm 1,18$ & $24,93 \pm 1,27$ & $22,80 \pm 1,70$ & $23,50 \pm 1,83$ \\
\hline \hline & \multicolumn{5}{c}{ Mujeres (Media \pm DS) } \\
nyyyyyy & M1 & M2 & M3 & M4 & Total \\
\cline { 2 - 6 } Número de Sujetos & 3 & 8 & 9 & 9 & 29 \\
Edad (años) & $27,67 \pm 5,69$ & $28,50 \pm 10,36$ & $24,78 \pm 7,29$ & $24,78 \pm 8,26$ & $26,10 \pm 8,18$ \\
Peso (kg) & $53,13 \pm 5,33$ & $51,86 \pm 4,71$ & $56,89 \pm 3,95$ & $59,34 \pm 3,26$ & $55,88 \pm 4,97$ \\
Estatura (cm) & $158,10 \pm 0,53$ & $161,11 \pm 1,54$ & $165,59 \pm 1,43$ & $171,14 \pm 2,53$ & $165,30 \pm 4,96$ \\
Longitud de Pierna (cm) & $82,00 \pm 1,00$ & $82,25 \pm 2,02$ & $86,26 \pm 1,78$ & $88,33 \pm 2,78$ & $85,36 \pm 3,36$ \\
Anchura de Pelvis (cm) & $20,50 \pm 1,00$ & $21,19 \pm 1,85$ & $22,17 \pm 1,75$ & $23,29 \pm 1,66$ & $22,07 \pm 1,88$ \\
\hline
\end{tabular}




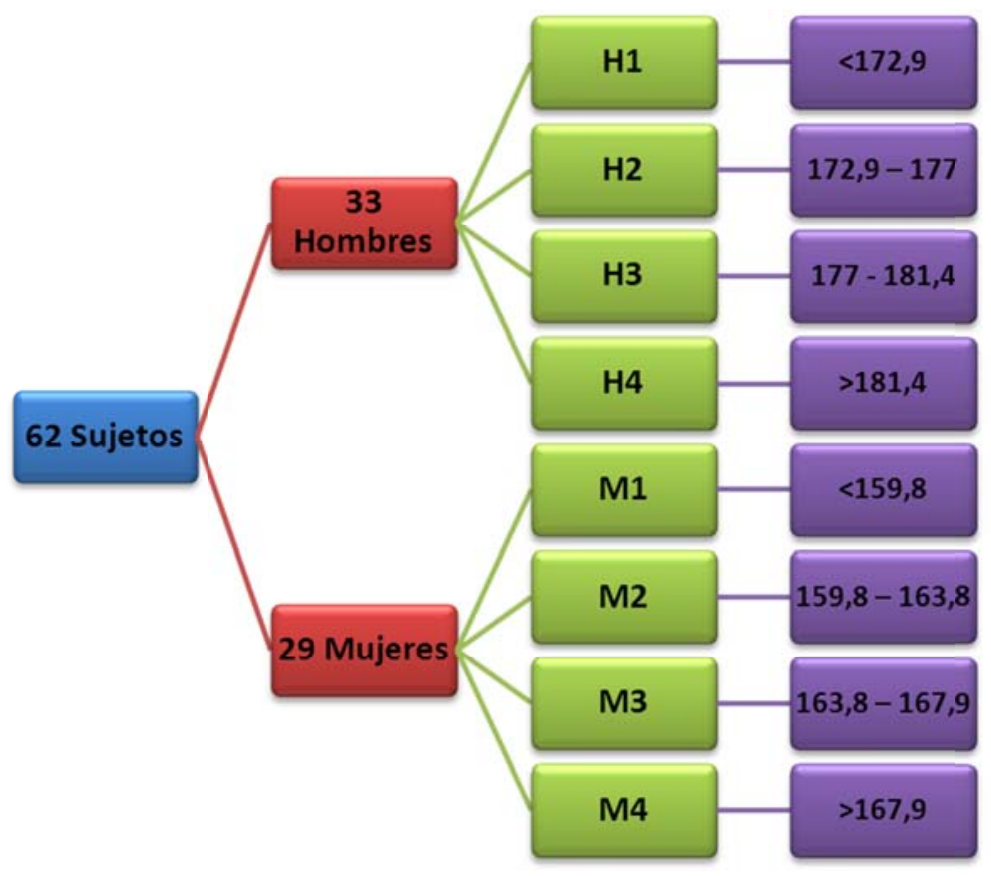

Figura 27. Distribución de la muestra en grupos de estatura

\subsubsection{Muestra sujetos sanos para prueba con dispositivo Hybrid (Estudio 2)}

De la muestra de 62 sujetos se seleccionaron aleatoriamente para las pruebas con el dispositivo Hybrid, para el Estudio 2, cinco hombres $(29,40 \pm 4,62$ años; $67,88 \pm 7,92 \mathrm{~kg}$ y $174,90 \pm 6,58 \mathrm{~cm})$ y cinco mujeres $(29,20 \pm 9,20$ años; $56,44 \pm 3,77 \mathrm{~kg}$ y $164,62 \pm 5,00 \mathrm{~cm})$ representantes de todos los grupos de estatura descritos en el patrón de la marcha normal. 
METOdOLOGÍA

\subsection{Instrumentos de Medida}

Todas las grabaciones se realizaron con un sistema 3D de captura de movimiento (Vicon Motion System, Oxford, UK) compuesto por 6 cámaras infrarrojas a $120 \mathrm{~Hz}$, como estudios previos describen (Benedetti, Manca, Ferraresi, Boschi, \& Leardini, 2011; Fukuchi, Arakaki, Veras Orselli, \& Duarte, 2010; Hallemans, Ortibus, Meire, \& Aerts, 2010; Schwartz, Rozumalski, \& Trost, 2008; Sliwinski, Sisto, Batavia, Chen, \& Forrest, 2004; Weiss et al., 2008;), y dispuestas a lo largo de un pasillo de marcha de 10 metros de longitud. En el espacio de captura se establecieron marcas en el suelo para señalar los 5 metros (entre punto A y punto B) que se utilizarían para cronometrar y determinar la velocidad de la marcha lenta (Figura 28).

El espacio de captura se calibró previamente a la toma de datos. La calibración estática determinó los ejes $x, y, z$ del espacio y el origen de coordenadas de los tres ejes. La calibración dinámica analizó la calidad de la captura y el error de las cámaras en la detección de la posición de los marcadores en movimiento. El error máximo admitido en la calibración de las cámaras se estableció en $2 \mathrm{~mm}$.

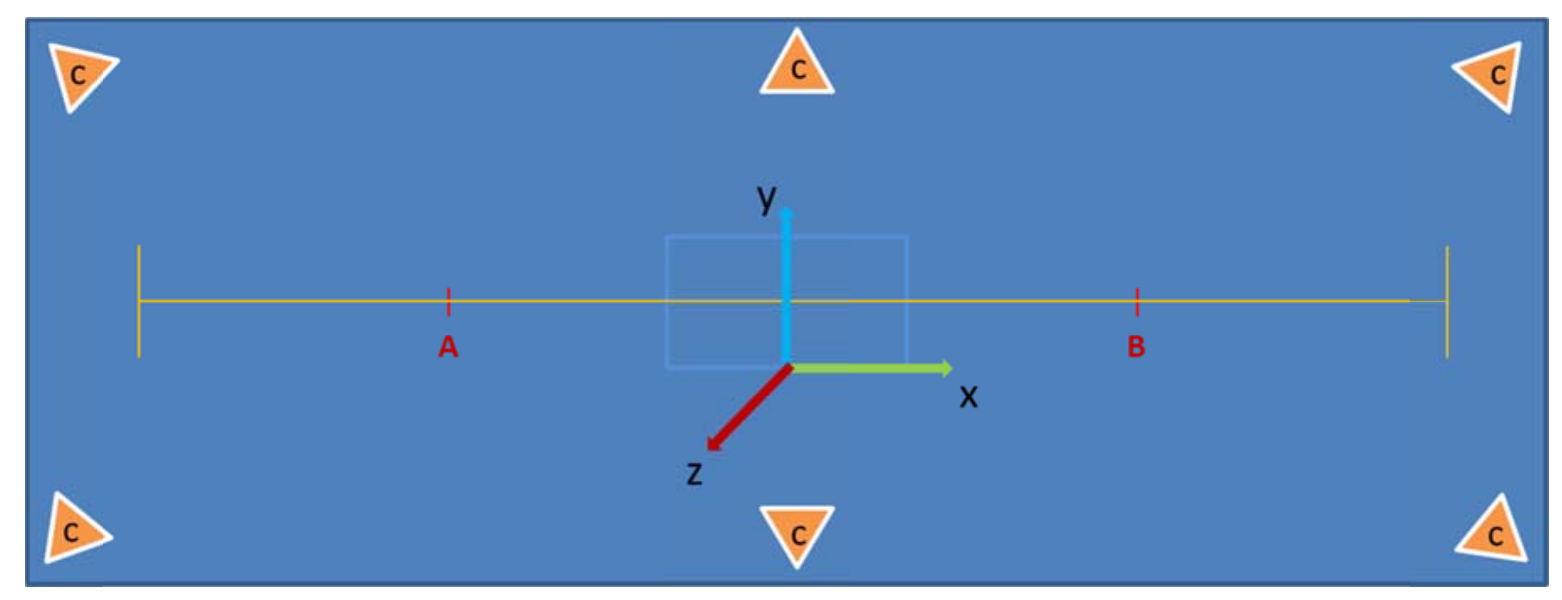

Figura 28. Esquema de espacio de captura 


\subsection{Adquisición de Datos}

\subsubsection{Estudio 1: Patrón de la Marcha a Velocidad Normal y Lenta}

\subsubsection{Protocolo}

Todos los sujetos voluntarios firmaron el consentimiento informado adjunto en el Anexo I aprobado por el Comité de Ética de la Universidad Politécnica de Madrid (Anexo II). Previo al análisis de la marcha se tomaron medidas antropométricas de los sujetos: peso (mass), altura (height), distancia entre espinas ilíacas anterosuperiores (EIAS) (Inter-asis distance), distancia entre EIAS y la proyección hacia anterior de la posición del trocánter (Asis-trocánter distance), longitud de pierna (desde EIAS a maléolo interno) (leg length) con una cinta métrica, y anchura de rodilla (knee width) y anchura de tobillo (ankle width) con un calibre (Anexo III).

Se colocaron sobre la piel del sujeto 19 marcadores reflectantes con una cinta adhesiva hipoalergénica siguiendo el modelo Plug in Gait de VICON para miembro inferior que se describe en el apartado "3.4.1.2. Modelo de marcadores y Modelo mecánico".

Una vez en la zona de registro de datos (pasillo de marcha), en primer lugar se grabó una repetición estática (Static) en la que se pedía al sujeto permanecer en el centro del espacio de captura con los brazos en cruz tres segundos. De este modo, se registraban todos los marcadores en estático para su utilización posterior en la identificación de los marcadores en las repeticiones dinámicas y como referencia del sistema sólido rígido en el modelo dinámico. A continuación se le solicitó al sujeto caminar descalzo a velocidad normal libremente seleccionada recorriendo de forma longitudinal el pasillo de marcha. En cada uno de los extremos se le dio la señal para el comienzo de la grabación de la repetición. Tras el registro de datos a velocidad normal se le requirió al sujeto caminar a 0,25 metros/segundo, lo que se traduce en que debía tardar aproximadamente 20 segundos en recorrer 5 metros del pasillo, aproximadamente al $20 \%$ de su velocidad normal y que corresponde con la velocidad a la que el dispositivo Hybrid reproduciría los patrones de la marcha. En cada uno de las repeticiones se cronometró el tiempo que el sujeto empleaba en recorrer 5 metros, determinando así si la velocidad correspondía con la deseada. De cada una de las velocidades se grabaron 3 repeticiones, con ciclo de la marcha derecho e izquierdo cada uno (Figura 29). 


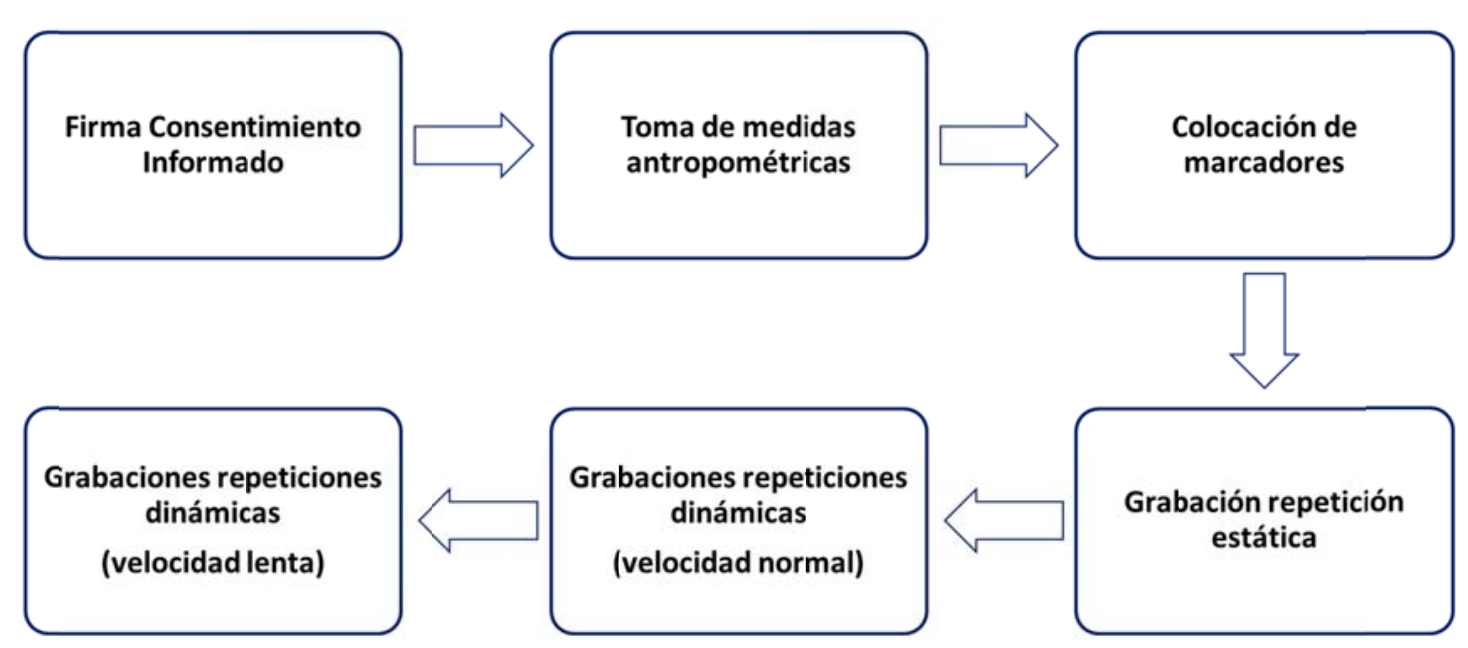

Figura 29. Protocolo de medida del Estudio 1

\subsubsection{Modelo de marcadores y Modelo mecánico}

El modelo de marcadores utilizado se compuso de un total de 19 marcadores reflectantes (14 $\mathrm{mm}$ diámetro) colocados de forma bilateral en puntos anatómicos establecidos por el modelo de miembro inferior Plug In Gait de VICON (Vicon, 2003). Se situaron marcadores en la parte posterior del talón (LHEE y RHEE), en las cabezas del 2ㅇ metatarsiano (LTOE y RTOE), en el maléolo externo de tobillos siguiendo el eje transmaleolar (LANK y RANK), la línea media lateral de pierna (LTIB y RTIB), el cóndilo externo de fémur sobre el eje de flexo-extensión (mediolateral) de la rodilla (LKNE y RKNE), la línea media lateral del musla (LTHI y RTHI), la parte anterior de espinas ilíacas anterosuperiores (LASI y RASI), las espinas ilíacas posterosuperiores (LPSI y RPSI), la apófisis espinosa de la 7ạ vértebra cervical (C7) y 10a vértebra dorsal (T10), y en la escotadura yugular (CLAV) (Figura 30). 


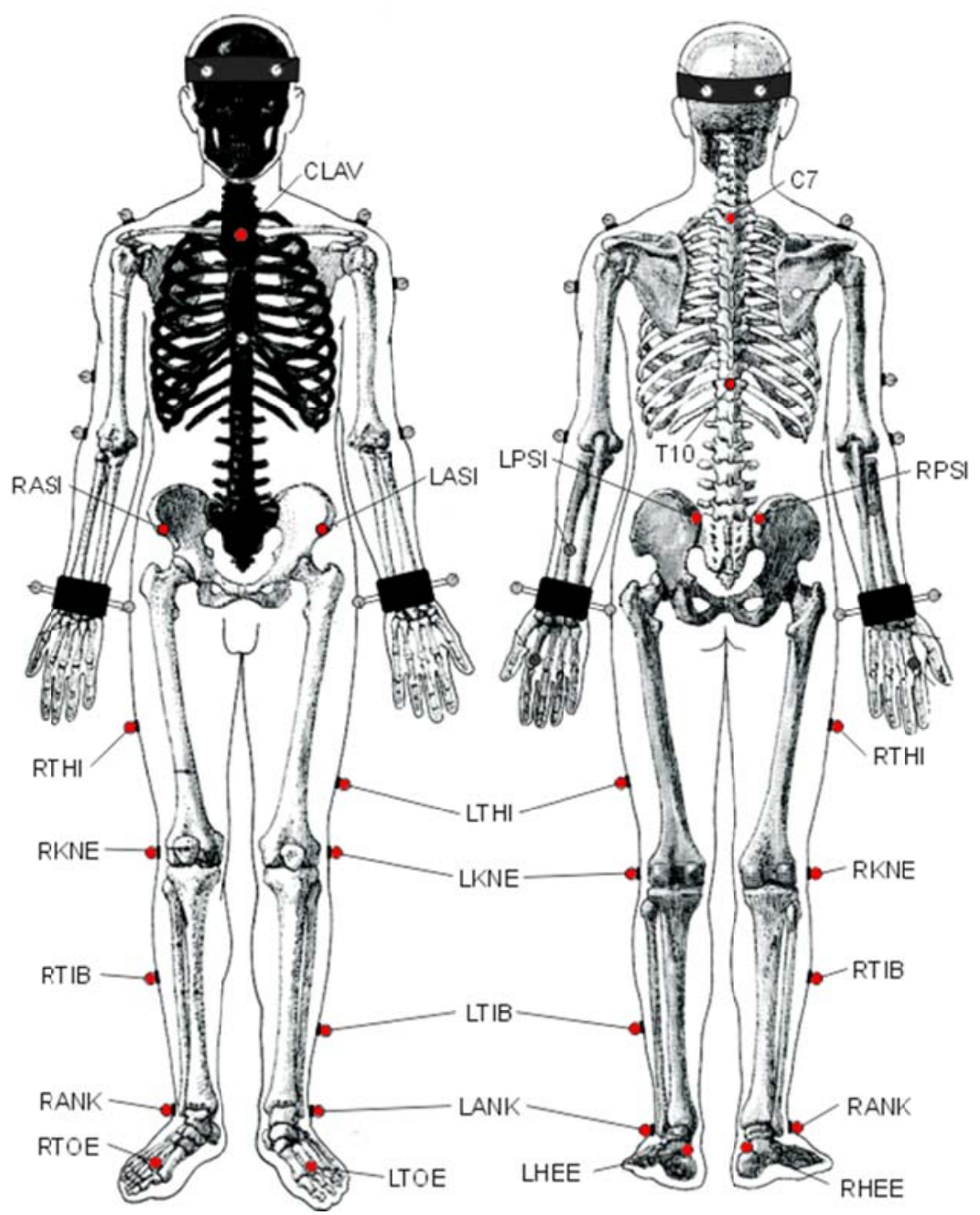

Figura 30. Modelo Plug in Gait para la colocación de marcadores en miembro inferior (tomado de Vicon, 2003)

El modelo mecánico definido por todos los marcadores descritos se representó con un sistema sólido rígido con segmentos corporales, definidos en pelvis, muslo, pierna y pie. El movimiento angular entre los segmentos en los ejes anteroposterior, mediolateral y vertical definieron los movimientos de cada articulación. Los cuatro marcadores situados en espinas ilíacas anterosuperiores (LASI y RASI) y posterosuperiores (LPSI y RPSI) delimitaron el segmento de la pelvis que permitió analizar el movimiento de la misma en el plano sagital, frontal y transversal. Desde la espina iliaca anterosuperior (LASI y RASI) hasta el marcador del cóndilo externo de la rodilla (LKNEE y RKNEE) se delimitó el segmento muslo. El segmento pierna estuvo comprendido entre el cóndilo externo de la rodilla (LKNEE y RKNEE) hasta el maléolo externo del tobillo (LANK y RANK) y el segmento pie entre la punta (LTOE y RTOE) al talón del pie (LHEE y RHEE) (Figura 31). 
Los centros articulares de rodilla y tobillo se determinaron por la posición del marcador externo y el ancho de la articulación. El centro articular de la cadera se definió a partir de la posición de los marcadores de la pelvis y de la línea media del muslo (LTHI y RTHI).

Los movimientos en el plano sagital entre el segmento pelvis y el segmento muslo alrededor del eje mediolateral de la cadera determinaron los movimientos de flexo-extensión de la cadera, los movimientos en el plano sagital entre el segmento muslo y el segmento pierna alrededor del eje mediolateral de la rodilla definieron los movimientos de flexo-extensión de la rodilla, y los movimientos en el plano sagital entre el segmento pierna y el segmento pie alrededor del eje mediolateral del tobillo determinaron los movimientos de flexo-extensión del tobillo. Los marcadores de talón (LHEE y RHEE) y punta del pie (LTOE y RTOE) determinaron el apoyo y despegue del pie, de la consecución de un ciclo y de la determinación de las fases y parámetros espaciotemporales de la marcha.
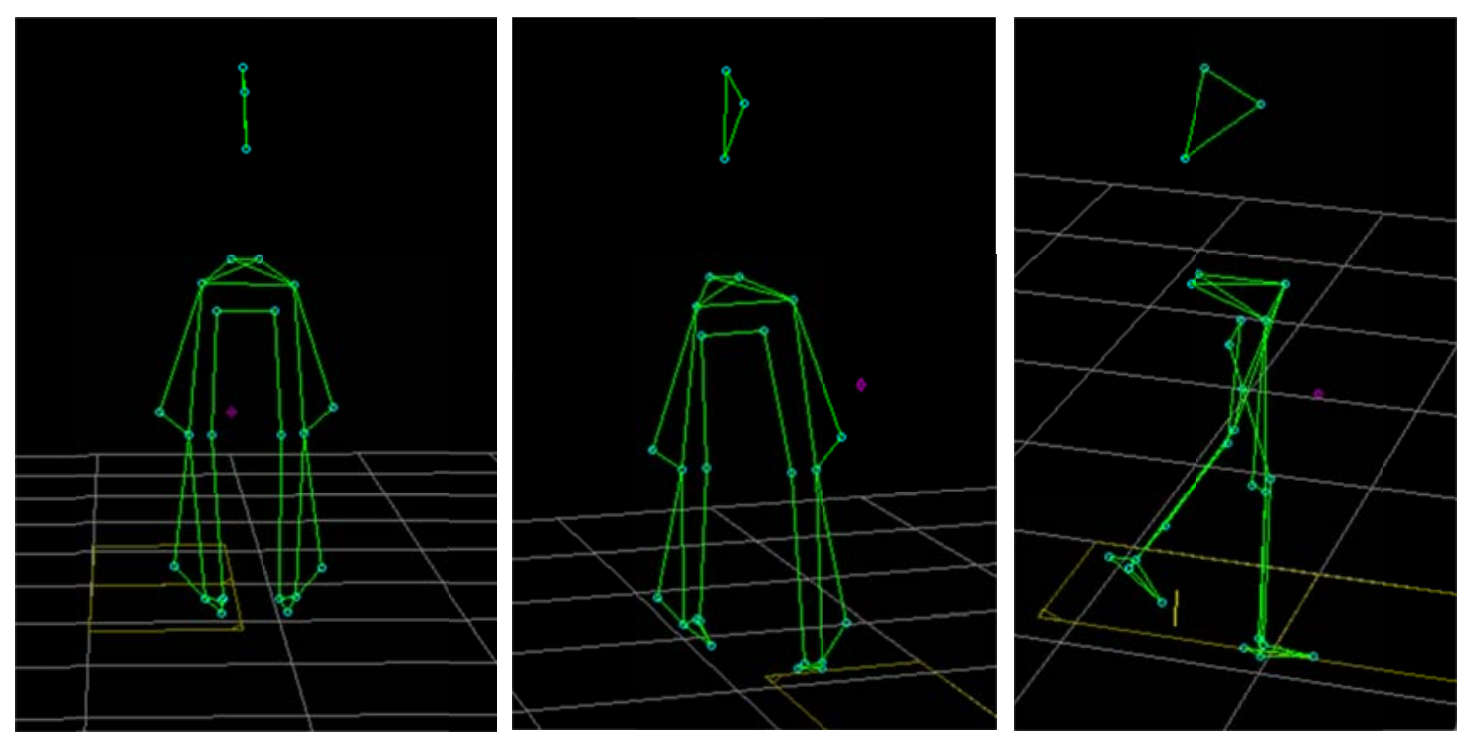

Figura 31. Modelo mecánico Plug in Gait

\subsubsection{Estudio 2: Análisis de la marcha con dispositivo Hybrid}

\subsubsection{Protocolo}

Para las pruebas con el dispositivo Hybrid se reprodujeron los patrones de la marcha a velocidad lenta $(25 \mathrm{~cm} / \mathrm{s})$. Los 10 sujetos portaron el dispositivo y realizaron pruebas con diferentes porcentajes de descarga de su peso (30\%, 50\% y $70 \%)$. Igualmente se grabaron tres repeticiones con ciclos derecho e izquierdo en cada una (Figura 32). 


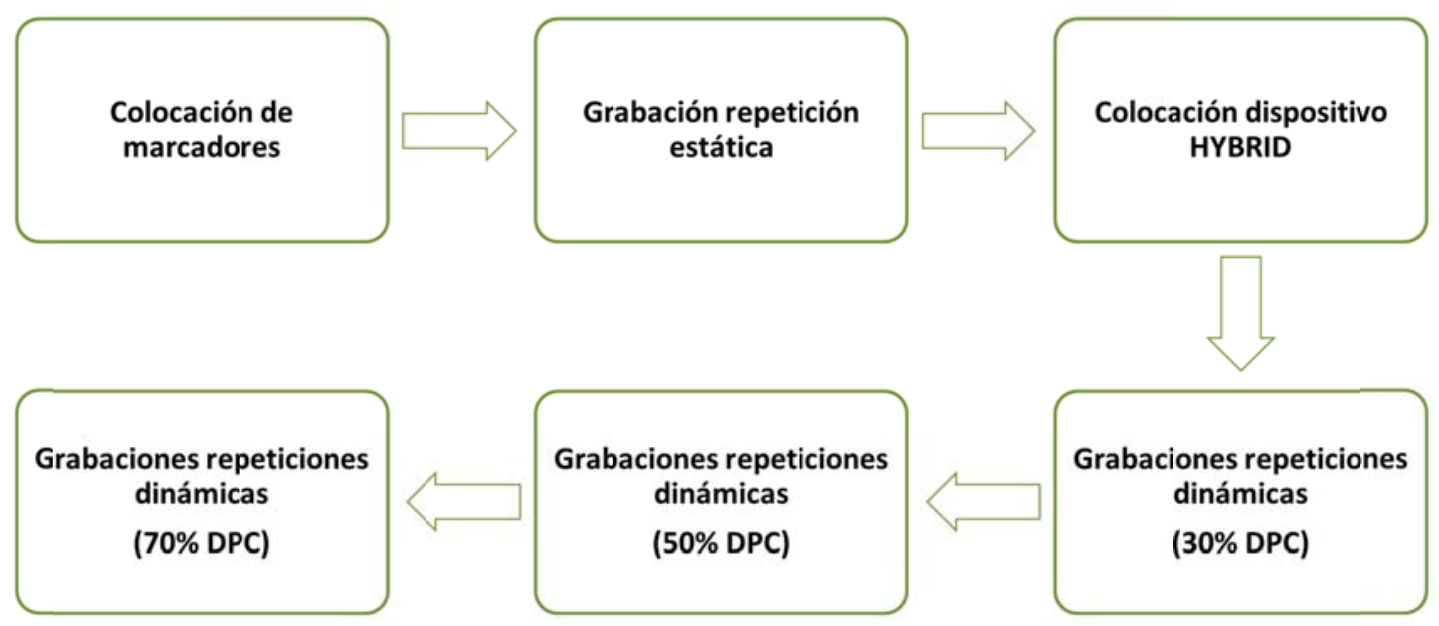

Figura 32. Protocolo de medida del Estudio 2

\subsubsection{Modelo de marcadores y Modelo mecánico}

En cada una de las repeticiones con el dispositivo Hybrid se utilizó un modelo de marcadores diseñado para adaptarse y ser compatible con el exoesqueleto. Durante las medidas estáticas (sin exoesqueleto) se colocaron 36 marcadores en puntos anatómicos de forma bilateral: en el talón (LHEE y RHEE), en las cabezas del 2 o metatarsiano (LTOE y RTOE); en el maléolo externo (LANK y RANK) y maléolo interno del tobillo (LANK2 y RANK2) determinando el centro articular del tobillo; en la línea media lateral de pierna (LTIB y RTIB); en el cóndilo externo (LKNE y RKNE) e interno del fémur (LKNE2 y RKNE2) definiendo el centro articular de la rodilla; en la línea media lateral del muslo (LTHI y RTHI), cuatro marcadores en la pelvis (espinas ilíacas anterosuperiores (LASI y RASI), espinas ilíacas posterosuperiores (LPSI y RPSI)) que definieron el centro de la articulación de la cadera y; dos marcadores en el trocánter mayor del fémur (LTROC y RTROC) y parte superior de articulación acromioclavicular (LSHO y RSHO). Además, se colocaron tres marcadores en la parte posterior del muslo (LMUS1, LMUS2, LMUS3 y RMUS1, RMUS2, RMUS3) que recordaban y redefinían el centro articular de la cadera y el segmento muslo durante las medidas dinámicas, así como otros tres marcadores en la parte posterior de la pierna (LGEM1, LGEM2, LGEM3 Y RGEM1, RGEM2, RGEM3) redefiniendo el centro articular de la rodilla y el tobillo y el segmento pierna.

En las mediciones dinámicas (con el exoesqueleto) el modelo se redujo a 28 marcadores. Se eliminaron los marcadores situados en el trocánter mayor (LTROC y RTROC), los marcadores en la línea media lateral del muslo y pierna (LTHI, RTHI, LTIB y RTIB), y los marcadores mediales y laterales de rodilla y tobillo (LKNE2, LKNE, RKNE2, RKNE, LANK2, LANK, RANK2 y RANK). Se añadieron seis marcadores en el centro de los motores para controlar la posición del 
METOdOLOGÍA

exoesqueleto (E_LHIP, E_LKNE, E_LANK y E_RHIP, E_RKNE, E_RANK) y se sustituyeron los marcadores de la pelvis (LASI, RASI, LPSI y RPSI) por otros marcadores sobre la cesta pélvica del exoesqueleto (E_LASI, E_RASI, E_LPSI y E_RPSI) (Gómez, López de Subijana, Urendes, \& Navarro, 2015) (Figura 33).
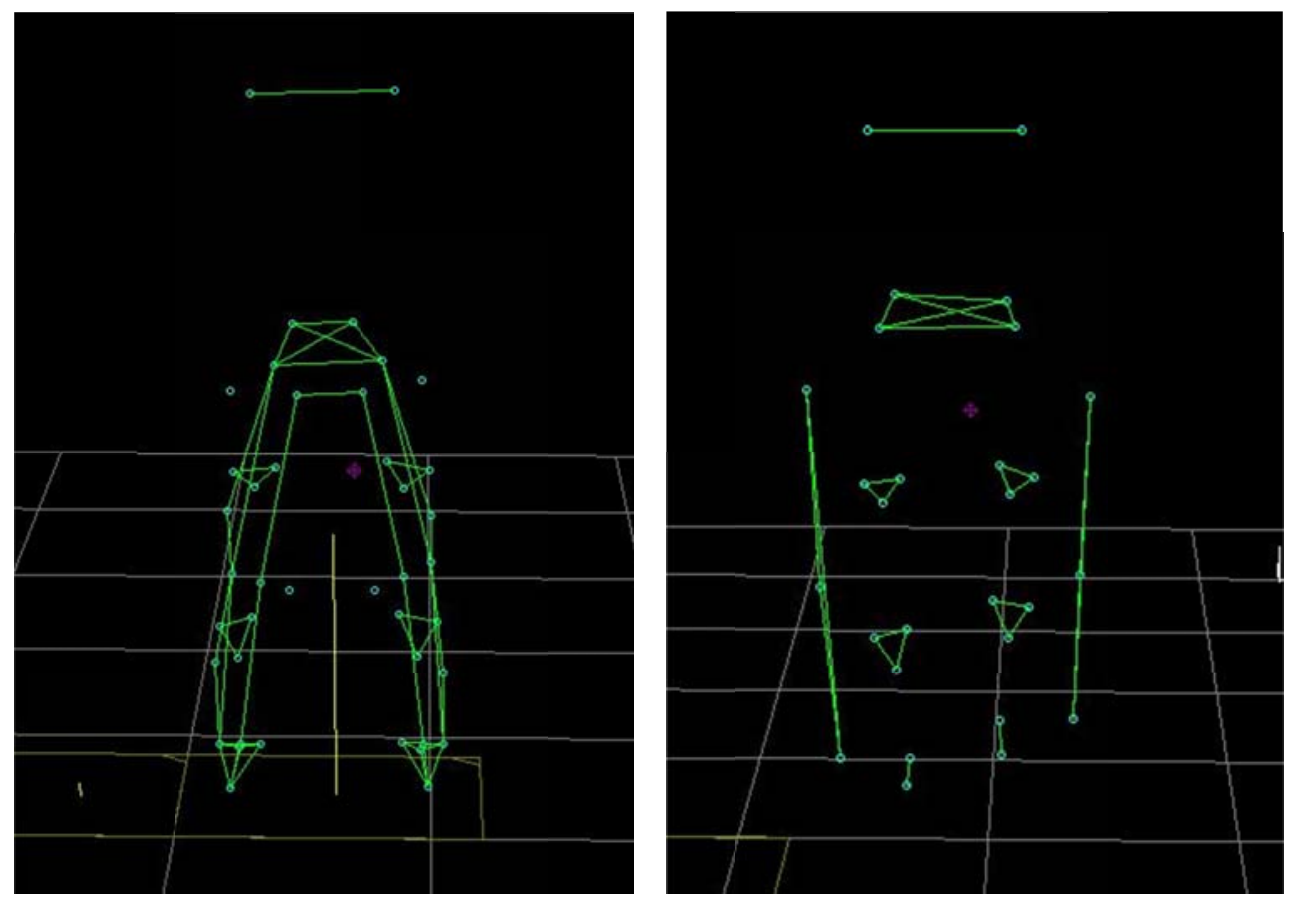

Figura 33. Modelo de marcadores estático y dinámico adaptado al exoesqueleto 


\subsection{Procesamiento de datos}

El procesamiento de los datos se realizó utilizando el software específico de Vicon System, incluyendo los programas Workstation y Bodybuilder. Fundamentalmente se utilizó el programa Workstation para el etiquetado de marcadores, revisión de trayectoria, filtrado y aplicación del modelo mecánico. En Bodybuilder se exportaron los archivos .txt con las trayectorias de diferentes marcadores o articulaciones.

\subsubsection{Medidas del sujeto}

Se introdujeron las medidas antropométricas tomadas durante el registro de datos para individualizar la reconstrucción del modelo a cada uno de los sujetos antes del etiquetado de marcadores. En Trial >>Subjects Measurements se introdujeron el peso del sujeto (mass), la altura (height), la distancia entre espinas ilíacas anterosuperiores (inter-asis distance), la longitud de pierna derecha e izquierda (leg lenght), la anchura de la rodilla derecha e izquierda (knee width), la anchura de tobillo derecho e izquierdo (ankle width) y la distancia entre la espina ilíaca anterosuperior y la proyección anterior del trocánter derecha e izquierda (asistrocanter distance) (Figura 34).

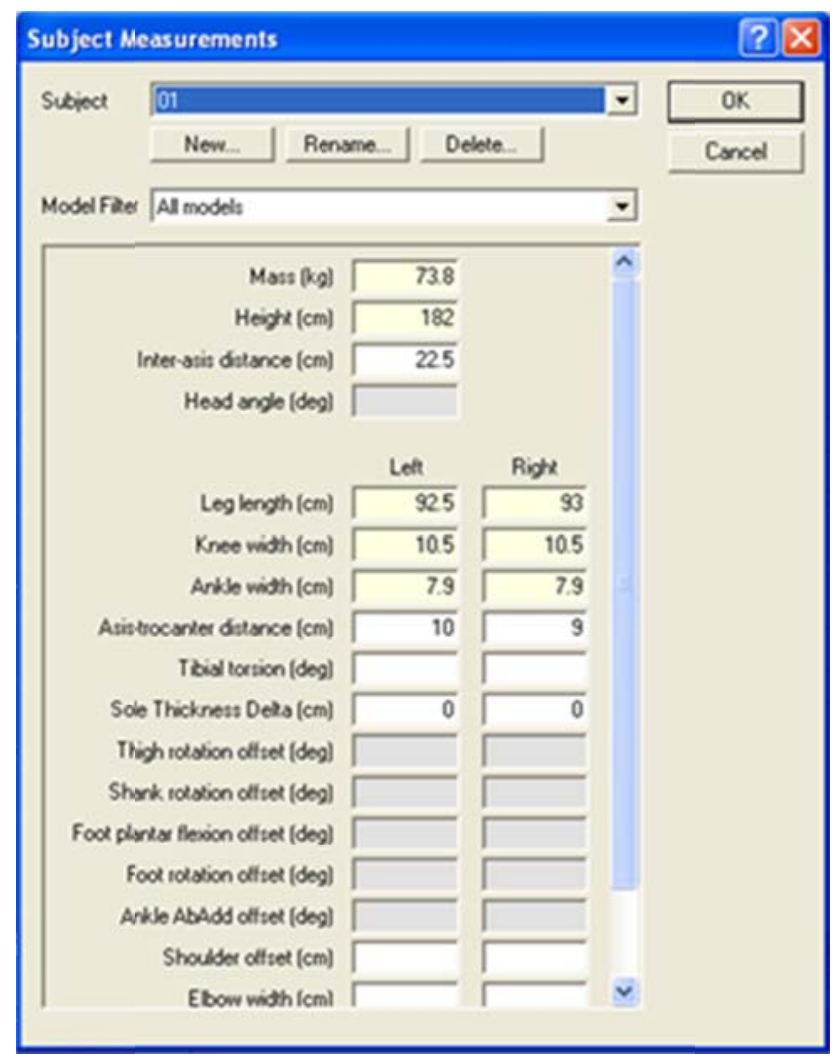

Figura 34. Workstation. Subject Measurements 


\subsubsection{Etiquetado de marcadores}

Previo al procesamiento de las repeticiones dinámicas de cada sujeto se etiquetaron los marcadores en la repetición estática (static) siguiendo el modelo Plug in Gait descrito con anterioridad. Posteriormente se nombraron los marcadores en cada una de las repeticiones dinámicas y se marcaron los eventos que delimitarían el ciclo de la marcha. Se identificó el primer apoyo de talón, despegue del pie y segundo apoyo de talón en pie derecho e izquierdo determinando así el ciclo derecho y ciclo izquierdo en cada una de las repeticiones (Figura 35).

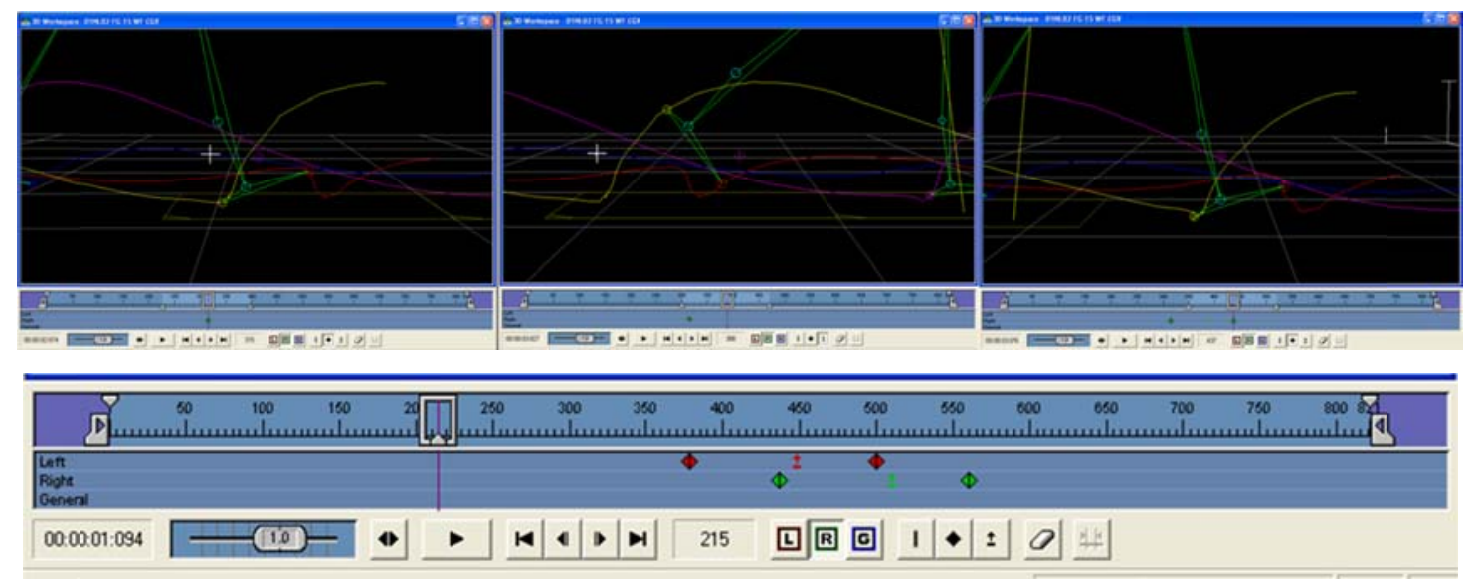

Figura 35. Workstation. Identificación de eventos en ciclo de la marcha

Una vez eliminados los elementos ajenos al modelo de marcadores (denominados brillos o ghosts) y revisados el recorrido armónico de cada una de las trayectorias de llos marcadores se aplicó el filtrado Woltring (1986) (Pipeline>>Apply Woltring filtering routine), para el suavizado de trayectorias y reconstrucción de tramos con pérdida de información (gapis) (Figura 36). Las repeticiones fueron cortadas 20 fotogramas antes del primer evento y 20 fotogramas después del último evento marcado. 


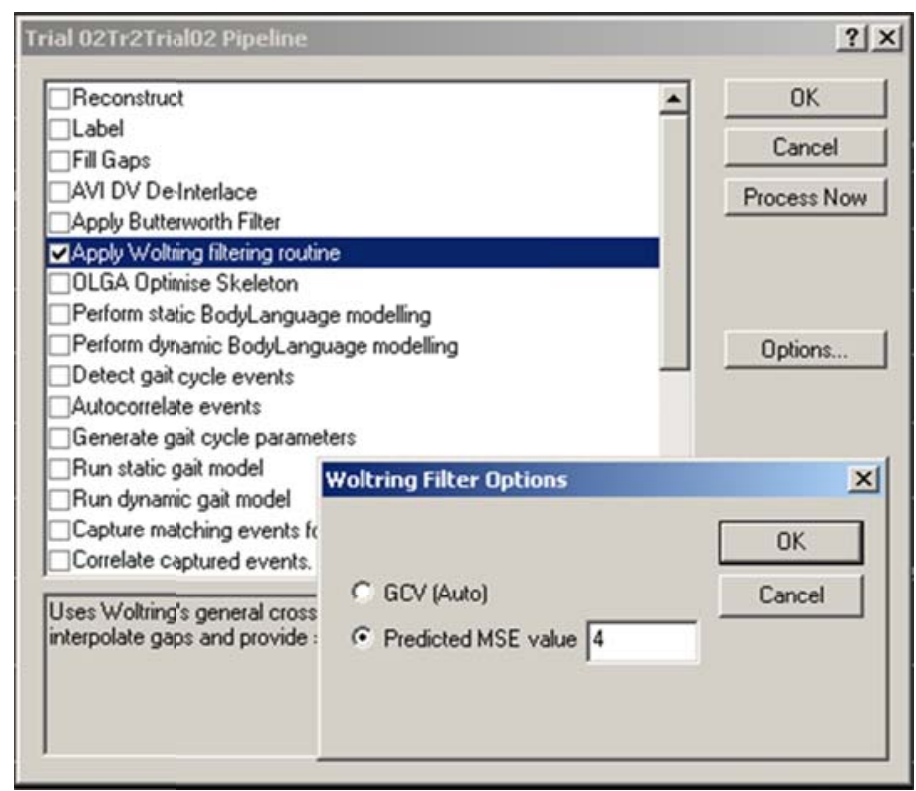

Figura 36. Workstation. Woltring Filtering Routine

\subsubsection{Modelo Mecánico}

En primer lugar se aplicó el modelo estático (Pipeline>> Run Static Modell) que se tomaría como referencia para reconstruir los centros articulares en el modelo dinámico, y posteriormente se aplicó el modelo dinámico en cada una de las repeticiones (Pipeline>>Run Dynamic Model) (Figuras 37 y 38 ).

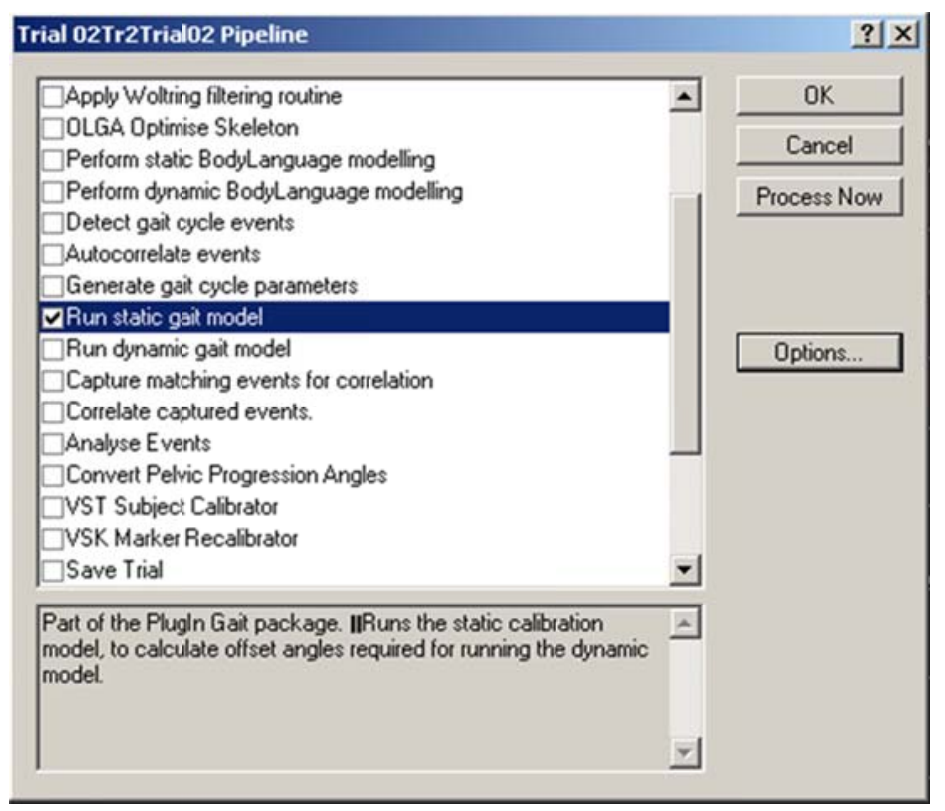

Figura 37. Workstation. Run Static Gait Model 


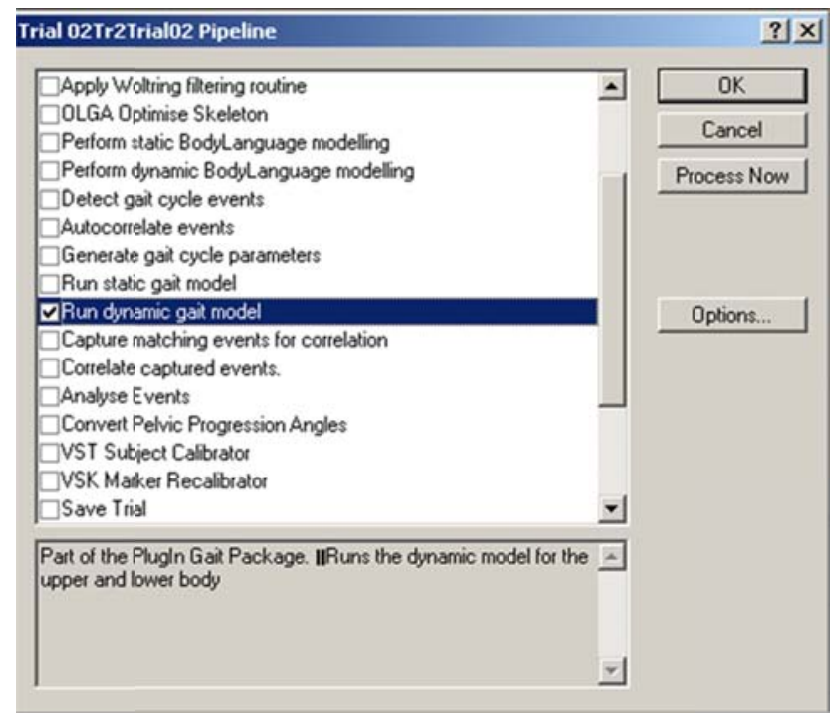

Figura 38. Workstation. Run Dynamic Gait Model

\subsubsection{Exportado de trayectorias}

Una vez se generó el modelo mecánico, se exportaron las trayectorias de las articulaciones y marcadores seleccionados. En el programa Bodybuilder se exportaron en el siguiente orden y en cada una de las repeticiones dinámicas (File>>Write ASCII File) las trayectorias de: el centro de gravedad del cuerpo (PELP), los marcadores situados en el talón izquierdo (LHEE) y punta del pie izquierdo (LTOE), los marcadores situados en el talón derecho (RHEE) y punta del pie derecho (RTOE), los ángulos de la pelvis (LPelvisAngles), los ángulos de cadera, rodilla y tobillo izquierdos (LHipAngles, LKneeAngles y LAnkleAngles) y los ángulos de cadera, rodilla y tobillo derechos (RHipAngles, RKneeAngles y LAnkleAngles) (Figura 39).

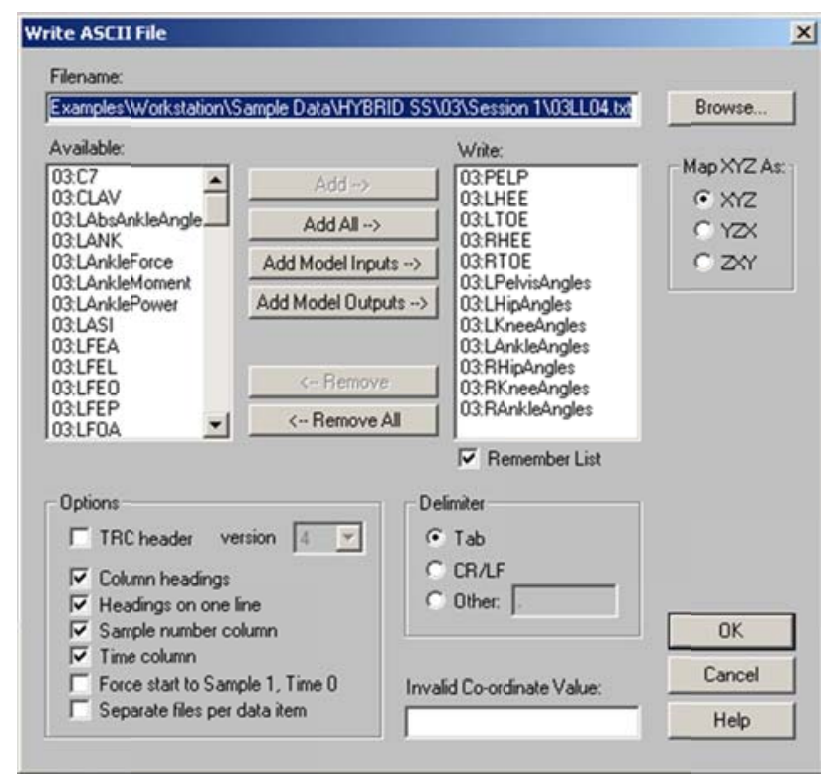

Figura 39. Bodybuilder. Exportado de trayectorias de marcadores y centros articulares 


\subsection{Variables}

Las variables espacio-temporales y cinemáticas fueron obtenidas de una media de tres ciclos de la marcha seleccionados de cada lado y normalizadas al 0-100\% del ciclo.

Las variables espacio-temporales que se estudiaron fueron: velocidad $(\mathrm{cm} / \mathrm{s})$, cadencia (pasos/min), longitud de paso $(\mathrm{cm})$, longitud de zancada $(\mathrm{cm})$, anchura de paso $(\mathrm{cm})$, porcentaje de apoyo en el ciclo (\%), porcentaje de oscilación en el ciclo (\%), tiempo de apoyo en el ciclo (s), tiempo de oscilación en el ciclo (s), tiempo de paso (s), tiempo de zancada (s), tiempo de apoyo monopodal (s) y tiempo de doble apoyo (s). Todas ellas, tal y como se muestra en la Tabla 3, fueron seleccionadas basándose en estudios previos de diferentes autores. Las velocidades, longitudes de paso y longitudes de zancada se normalizaron en función de la estatura y de la longitud de pierna. La anchura de paso se normalizó en función de la anchura de la pelvis, determinada por la distancia entre EIAS (Frimenko et al., 2015; Hof, 1996; Kirtley, 2006; Pierrynowski, \& Galea, 2001).

Los parámetros cinemáticos que se obtuvieron, también seleccionados según estudios previos (Tabla 4), fueron: el rango de movimiento del centro de gravedad en el plano frontal (movimiento lateral) $(\mathrm{mm})$ y en el plano sagital (movimiento cráneo-caudal) $(\mathrm{mm})$, el rango de movimiento de la pelvis en los tres planos $\left({ }^{\circ}\right)$ : sagital (anteversión y retroversión), frontal (basculación lateral) y transversal (rotación). En la cinemática de la flexoextensión de cadera, rodilla y tobillo se seleccionaron datos discretos (máximos, mínimos y apoyo inicial) de las curvas ángulo articular/porcentaje ciclo, siendo estos datos los que describían con mayor exactitud la curva. Se analizaron, por tanto, los grados de flexión máxima y mínima de cadera, rodilla y tobillo $\left({ }^{\circ}\right.$ ) y el instante del ciclo en el que sucedían (\% del ciclo), y los grados de flexión de cadera, rodilla y tobillo $\left({ }^{\circ}\right)$ en el instante del contacto del talón ( $0 \%$ del ciclo). 
Tabla 3. Relación de variables dependientes espaciotemporales estudiadas por otros autores y en el presente estudio

\begin{tabular}{|c|c|c|c|c|c|c|c|c|c|c|c|c|c|c|}
\hline Autores & Año & País & $\begin{array}{l}\frac{0}{\pi} \\
\frac{\pi}{2} \\
\frac{0}{0} \\
\frac{0}{2}\end{array}$ & $\begin{array}{l}\frac{\pi}{U} \\
\frac{C}{0} \\
\frac{\pi}{0}\end{array}$ & 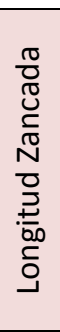 & 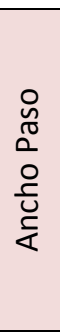 & 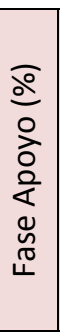 & 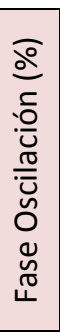 & 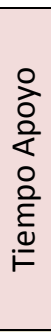 & 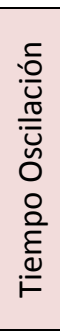 & 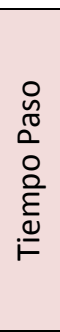 & 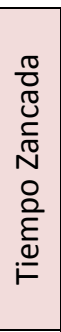 & 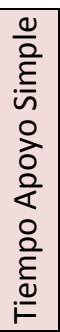 & $\begin{array}{l}\frac{0}{0} \\
0 \\
0 \\
0 \\
0 \\
0 \\
\frac{0}{0} \\
0 \\
0 \\
\frac{0}{\varepsilon} \\
\stackrel{0}{0}\end{array}$ \\
\hline $\begin{array}{l}\text { Benedetti, Catani, Leardini, } \\
\text { Pignotti, \& Giannini }\end{array}$ & 1998 & Italia & $*$ & $*$ & $*$ & & $*$ & & $*$ & & & & & \\
\hline $\begin{array}{l}\text { Threlkeld, Cooper, Monger, } \\
\text { Craven, \& Haupt }\end{array}$ & 2003 & USA & & $*$ & $*$ & & & & * & $*$ & & & & $*$ \\
\hline Sliwinski et al. & 2004 & USA & $*$ & & & & & & & & & & & \\
\hline Weiss et al. & 2008 & Suecia & $*$ & $*$ & $*$ & & & & & & & & & \\
\hline Hallemans et al. & 2010 & Bélgica & & $*$ & $*$ & $*$ & & & $*$ & & & & & \\
\hline $\begin{array}{c}\text { Beaulieu, Lamontagne, \& } \\
\text { Beaulé }\end{array}$ & 2010 & Canadá & $*$ & $*$ & $*$ & & & & & & & & & \\
\hline $\begin{array}{c}\text { Watt, Franz, Jackson, } \\
\text { Dicharry, Riley, \& Kerrigan }\end{array}$ & 2010 & USA & $*$ & $*$ & $*$ & & & & & & $*$ & & & \\
\hline $\begin{array}{c}\text { Linley, Sled, Culham, \& } \\
\text { Deluzio }\end{array}$ & 2010 & Canadá & $*$ & $*$ & $*$ & & & & & & & & & $*$ \\
\hline Benedetti et al. & 2011 & Italia & $*$ & $*$ & $*$ & & $*$ & & & & & & & \\
\hline $\begin{array}{c}\text { Sagawa, Turcot, Armand, } \\
\text { Thevenon, Vuillerme, \& } \\
\text { Watelain }\end{array}$ & 2011 & Francia & $*$ & $*$ & $*$ & $*$ & $*$ & $*$ & $*$ & $*$ & $*$ & $*$ & $*$ & $*$ \\
\hline $\begin{array}{c}\text { Bovi, Rabuffetti, Mazzoleni, \& } \\
\text { Ferrarin }\end{array}$ & 2011 & Italia & $*$ & $*$ & $*$ & & & & $*$ & & & & & $*$ \\
\hline $\begin{array}{l}\text { Gil-Agudo, Pérez-Nombela, } \\
\text { Forner-Cordero, Pérez-Rizo, } \\
\text { Crespo-Ruiz, \& del Ama- } \\
\text { Espinosa }\end{array}$ & 2011 & España & $*$ & * & $*$ & & * & & & & $*$ & & * & $*$ \\
\hline
\end{tabular}


Tabla 4. Relación de variables dependientes cinemáticas estudiadas por otros autores y en el presente estudio

\begin{tabular}{|c|c|c|c|c|c|c|c|c|c|c|c|}
\hline Autor & Año & Pais & 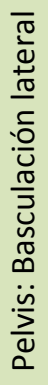 & 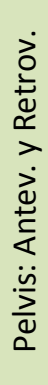 & 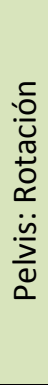 & $\begin{array}{l}\widehat{\bar{U}} \\
\frac{\pi}{0} \\
\frac{0}{0} \\
\frac{\pi}{0} \\
\simeq \\
\frac{0}{x} \\
\frac{0}{4}\end{array}$ & 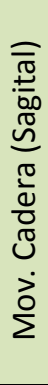 & 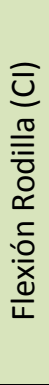 & 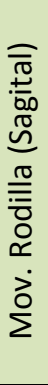 & 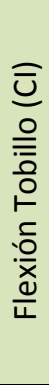 & 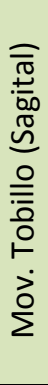 \\
\hline Benedetti et al. & 1998 & Italia & $*$ & $*$ & $*$ & $*$ & $*$ & $*$ & $*$ & $*$ & $*$ \\
\hline Threlkeld et al. & 2003 & USA & $*$ & $*$ & $*$ & $*$ & $*$ & $*$ & $*$ & $*$ & $*$ \\
\hline Schwartz et al. & 2008 & USA & $*$ & $*$ & $*$ & & $*$ & & $*$ & $*$ & $*$ \\
\hline Hallemans et al. & 2010 & Bélgica & $*$ & & $*$ & $*$ & $*$ & $*$ & $*$ & $*$ & $*$ \\
\hline Watt et al. & 2010 & USA & $*$ & & $*$ & & $*$ & & $*$ & $*$ & $*$ \\
\hline Linley et al. & 2010 & Canadá & $*$ & & & & & & & & \\
\hline Bovi et al. & 2011 & Italia & $*$ & $*$ & $*$ & & $*$ & & $*$ & $*$ & $*$ \\
\hline Gil Agudo et al. & 2011 & España & $*$ & $*$ & $*$ & $*$ & $*$ & $*$ & $*$ & $*$ & $*$ \\
\hline
\end{tabular}




\subsection{Tratamiento de datos}

Todos los análisis estadísticos se realizaron con el software SPSS v.21 (SPSS Inc., Chicago, IL, United States). Se calcularon medias y desviaciones estándar (Media \pm DS) y el $95 \%$ de intervalo de confianza (IC). El nivel alfa de significación se estableció en 0,05 para todas las pruebas estadísticas.

\subsubsection{Patrón de la marcha normal}

Para establecer los patrones de marcha normal se realizaron comparaciones de medias entre grupos de estatura de forma separada por sexo (Kruskal-Wallis). A continuación se analizaron las diferencias entre hombres y mujeres (U Mann-Whitney). Todas las pruebas utilizadas fueron no paramétricas ya que algunas de las variables no se correspondían con una distribución normal (Kolmogorov-Smirnov). Además, en las variables que se presentaron diferencias significativas por grupos de estatura, y por lo tanto no se consideró un único patrón de la marcha por sexo, se analizó la interacción entre sexo y estatura usando ANOVA de 2 factores (Figura 40).

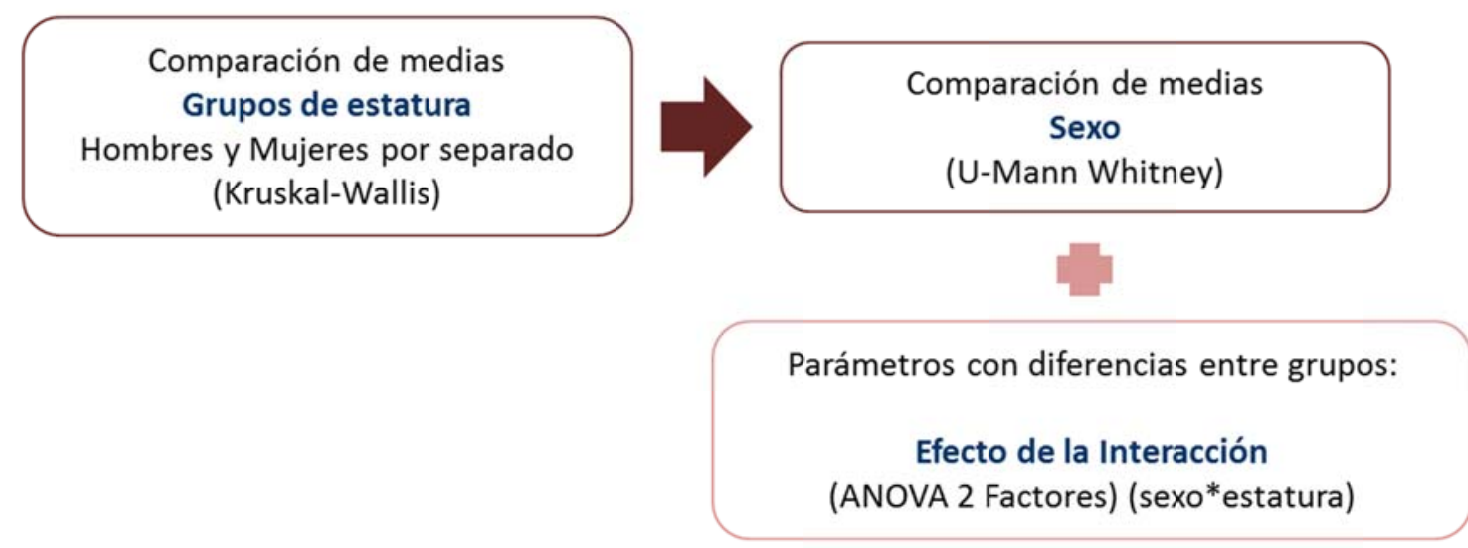

Figura 40. Análisis estadístico para establecer los patrones normales de la marcha

\subsubsection{Comparación del patrón velocidad normal y patrón velocidad lenta}

Se utilizó una prueba ANOVA de 2 factores de medidas repetidas (grupo de estatura y velocidad) para comparar el patrón a velocidad normal con el patrón a velocidad lenta de la marcha entre grupos de estatura. Los hombres y mujeres fueron analizados de forma independiente. 
3.7.3. Comparación del patrón con los porcentajes de descarga de dispositivo Hybrid con el patrón velocidad lenta

Se realizó un análisis no paramétrico para muestras relacionadas (Friedman) entre los porcentajes de descarga de peso al 30\%, 50\% y 70\% y el patrón a velocidad lenta. Los hombres y mujeres fueron analizados de forma independiente. 

4. Resultados 

A continuación se muestran los resultados obtenidos en el presente estudio. En primer lugar se presentarán los resultados de la marcha a velocidad normal en hombres y mujeres y por grupos de estatura (objetivo 1); en segundo lugar los resultados de la comparación entre la marcha a velocidad normal y a velocidad lenta, igualmente en hombres y mujeres y por grupos de estatura (objetivo 2); y, por último, los resultados de la comparación entre el patrón de la marcha a velocidad lenta y el patrón de la marcha reproducido por el exoesqueleto al 30\%, $50 \%$ y $70 \%$ de descarga de peso corporal, en hombres y mujeres de manera independiente (objetivo 3). En cada uno de los apartados los resultados se organizaron en el mismo orden: parámetros espacio-temporales (velocidad y cadencia, distancias y tiempos) y parámetros cinemáticos (pelvis y centro de gravedad, cadera, rodilla y tobillo).

\subsection{Análisis de las diferencias en la Marcha Normal en función del sexo y de la estatura de los sujetos}

Cada uno de los resultados se analizó en primer lugar entre los grupos de estatura de cada sexo $(\mathrm{H} 1, \mathrm{H} 2, \mathrm{H} 3$ y $\mathrm{H} 4$ para los hombres y $\mathrm{M} 1, \mathrm{M} 2, \mathrm{M} 3$ y $\mathrm{M} 4$ para las mujeres) y a continuación entre ambos grupos en función del sexo (hombres y mujeres).

\subsubsection{Parámetros espacio-temporales}

\subsubsection{Velocidad y cadencia}

Los hombres no presentaron diferencias entre los grupos por estatura, en los valores de velocidad $(\mathrm{cm} / \mathrm{s})(p=0,167)$, velocidad normalizada en función de la estatura $(p=0,157)$, velocidad normalizada en función de la longitud de pierna $(p=0,247)$ y la cadencia (pasos $/ \mathrm{min}$ ) $(p=0,582)$ (Tabla 5).

Las mujeres tampoco mostraron diferencias entre los grupos por estatura en las mismas variables $(p=0,219$ para velocidad, $p=0,111$ para velocidad normalizada en función de la estatura, $p=0,069$ para velocidad normalizada en función de la longitud de pierna, $p=0,112$ para cadencia) (Tabla 5).

No aparecieron diferencias entre hombres y mujeres en los valores absolutos de velocidad $(p=0,256)$. La velocidad normalizada en función de la estatura $(p=0,012)$, la velocidad normalizada en función de la longitud de pierna $(p=0,013)$ y la cadencia $(p=0,025)$ fueron mayores en las mujeres que en los hombres (Tabla 6). 
Tabla 5. Parámetros Espacio-temporales: Velocidad y Cadencia (Grupos por estatura)

\begin{tabular}{|c|c|c|c|c|c|c|c|c|c|}
\hline & \multicolumn{2}{|c|}{$\mathrm{H} 1$} & \multicolumn{2}{|c|}{$\mathrm{H} 2$} & \multicolumn{2}{|c|}{ H3 } & \multicolumn{2}{|c|}{$\mathrm{H} 4$} & \multirow{2}{*}{$x^{2}(3)$} \\
\hline & Media \pm DS & IC $95 \%$ & Media \pm DS & IC $95 \%$ & Media \pm DS & IC $95 \%$ & Media \pm DS & IC $95 \%$ & \\
\hline Velocidad $(\mathrm{cm} / \mathrm{s})$ & $125,72 \pm 10,84$ & $117,38-134,05$ & $132,77 \pm 14,61$ & $119,26-146,28$ & $118,65 \pm 17,86$ & $102,14-165,17$ & $143,05 \pm 27,36$ & $123,47-162,62$ & 5,07 \\
\hline Velocidad /Estatura & $0,74 \pm 0,06$ & $0,69-0,79$ & $0,76 \pm 0,08$ & $0,68-0,83$ & $0,66 \pm 0,10$ & $0,57-0,76$ & $0,78 \pm 0,15$ & $0,67-0,89$ & 5,21 \\
\hline Velocidad /Longitud Pierna & $1,44 \pm 0,14$ & $1,34-1,55$ & $1,46 \pm 0,17$ & $1,31-1,62$ & $1,29 \pm 0,24$ & $1,07-1,51$ & $1,49 \pm 0,30$ & $1,27-1,70$ & 4,14 \\
\hline \multirow[t]{3}{*}{ Cadencia (pasos/min) } & $113,18 \pm 5,47$ & $108,98-117,39$ & $113,00 \pm 8,44$ & $105,19-120,80$ & $108,83 \pm 10,63$ & $99,00-118,66$ & $116,21 \pm 10,37$ & $108,79-123,63$ & 1,95 \\
\hline & \multicolumn{2}{|c|}{ M1 } & \multicolumn{2}{|c|}{ M2 } & \multicolumn{2}{|c|}{ M3 } & \multicolumn{2}{|c|}{ M4 } & 2 \\
\hline & Media \pm DS & IC $95 \%$ & Media \pm DS & IC 95\% & Media \pm DS & IC $95 \%$ & Mean \pm SD & $95 \% \mathrm{Cl}$ & \\
\hline Velocidad $(\mathrm{cm} / \mathrm{s})$ & $116,29 \pm 21,93$ & $61,83-170,76$ & $143,48 \pm 17,43$ & $128,91-158,06$ & $136,88 \pm 23,88$ & $118,74-155,02$ & $128,53 \pm 14,43$ & $117,45-139,62$ & 4,42 \\
\hline Velocidad /Estatura & $0,74 \pm 0,14$ & $0,40-1,07$ & $0,89 \pm 0,11$ & $0,80-0,98$ & $0,83 \pm 0,14$ & $0,72-0,94$ & $0,75 \pm 0,09$ & $0,68-0,82$ & 6,02 \\
\hline Velocidad /Longitud Pierna & $1,42 \pm 0,28$ & $0,73-2,11$ & $1,74 \pm 0,21$ & $1,57-1,92$ & $1,59 \pm 0,27$ & $1,38-1,79$ & $1,46 \pm 0,19$ & $1,31-1,60$ & 7,09 \\
\hline Cadencia (pasos/min) & $111,97 \pm 13,68$ & $77,98-145,96$ & $123,55 \pm 6,99$ & $117,70-129,39$ & $120,04 \pm 11,26$ & $111,39-128,70$ & $112,63 \pm 8,94$ & $105,76-119,50$ & 5,98 \\
\hline
\end{tabular}

Hombres: H1 (<25\%), H2 (25\%-50\%), H3 (50\%-75\%), H4 (>75\%); Mujeres: M1 (<25\%), M2 (25\%-50\%), M3 (50\%-75\%), M4 (>75\%)

Tabla 6. Parámetros Espacio-temporales: Velocidad y Cadencia (Hombres y Mujeres)

\begin{tabular}{|c|c|c|c|c|c|}
\hline & \multicolumn{2}{|c|}{ Hombres } & \multicolumn{2}{|c|}{ Mujeres } & \multirow{2}{*}{ z } \\
\hline & Media \pm DS & IC $95 \%$ & Media \pm DS & IC $95 \%$ & \\
\hline Velocidad & $130,97 \pm 20,66$ & $123,86-138,90$ & $133,98 \pm 20,01$ & $126,37-141,59$ & $-0,66$ \\
\hline Velocidad/Estatura ${ }^{\dagger}$ & $0,74 \pm 0,11$ & $0,70-0,78$ & $0,81 \pm 0,13$ & $0,76-0,86$ & $-2,26$ \\
\hline Velocidad/Long. Pierna † & $1,43 \pm 0,23$ & $1,35-1,52$ & $1,57 \pm 0,25$ & $1,48-1,67$ & $-2,24$ \\
\hline Cadencia ${ }^{\dagger}$ & $113,14 \pm 8,90$ & $110,51-116,72$ & $117,87 \pm 10,39$ & $113,92-121,83$ & $-1,98$ \\
\hline
\end{tabular}

$+p<0,05$ (diferencias entre hombres y mujeres) 


\subsubsection{Distancias}

La longitud de paso $(\mathrm{cm})$ se presentó diferente $(p=0,047)$ en los grupos de estatura de los hombres, siendo los valores en el grupo H4 mayores que los del grupo H3 $(p=0,011)$. La longitud de paso normalizada en función de la estatura $(p=0,107)$ y normalizada en función de la longitud de pierna $(p=0,138)$ no mostraron diferencias entre los grupos de estatura de los hombres. La longitud de zancada $(\mathrm{cm})$ presentó diferencias $(p=0,035)$ en los grupos por estatura de los hombres, teniendo valores mayores en el grupo $\mathrm{H} 4$ que en los grupos $\mathrm{H} 1$ $(p=0,037)$ y $H 3(p=0,010)$. No se mostraron diferencias en la longitud de zancada normalizada en función de la estatura $(p=0,095)$, ni en la zancada normalizada en función de la longitud de pierna $(p=0,141)$. La anchura de paso $(c m)(p=0,354)$ y la anchura de paso normalizada en función de la anchura de pelvis $(p=0,321)$ no presentaron diferencias entre los grupos de estatura de los hombres (Tabla 7).

Ninguno de los parámetros referentes a distancias descritas anteriormente para los grupos de estatura de los hombres, presentaron diferencias para los grupos de estatura de las mujeres ( $p=0,108$ para longitud de paso, $p=0,106$ para longitud de paso normalizada en función de la estatura, $p=0,074$ para longitud de paso normalizada en función de la longitud de pierna, $p=0,207$ para longitud de zancada, $p=0,152$ para longitud de zancada normalizada en función de la estatura, $p=0,095$ para longitud de zancada normalizada en función de la longitud de pierna, $p=0,205$ para la anchura de paso y $p=0,107$ para la anchura de paso normalizada en función a la anchura de la pelvis) (Tabla 7).

En la comparativa por sexo la longitud de paso normalizada en función de la estatura $(p=0,013)$, la longitud de paso normalizada en función de la longitud de pierna $(p=0,011)$, la longitud de zancada normalizada en función de la estatura $(p=0,023)$ y la longitud de zancada normalizada en función de la longitud de pierna $(p=0,015)$ mostraron valores mayores en mujeres que en hombres. La anchura de paso $(p<0,001)$ y la anchura de paso normalizada en función de la anchura de la pelvis $(p=0,011)$ fueron mayores en hombres que en mujeres. La longitud de paso $(p=0,149)$ y la longitud de zancada ( $\sin$ normalizar) $(p=0,176)$ no presentaron diferencias en función del sexo (Tabla 8). 
Tabla 7. Parámetros Espacio-temporales: Distancias (Grupos por estatura)

\begin{tabular}{|c|c|c|c|c|c|c|c|c|c|}
\hline & \multicolumn{2}{|c|}{$\mathrm{H} 1$} & \multicolumn{2}{|c|}{$\mathrm{H} 2$} & \multicolumn{2}{|c|}{$\mathrm{H3}$} & \multicolumn{2}{|c|}{$\mathrm{H} 4$} & \multirow{2}{*}{$\chi^{2}(3)$} \\
\hline & Media \pm DS & IC $95 \%$ & Media \pm DS & IC $95 \%$ & Media \pm DS & IC $95 \%$ & Media \pm DS & IC $95 \%$ & \\
\hline Longitud Paso $(\mathrm{cm})^{*}$ & $66,89 \pm 3,14$ & $64,48-69,30$ & $70,41 \pm 5,44$ & $65,38-75,44$ & $65,28 \pm 4,20$ & $61,39-69,17$ & $73,71 \pm 8,52$ & $67,62-79,80$ & 7,95 \\
\hline Long. Paso/Estatura & $0,39 \pm 0,02$ & $0,38-0,41$ & $0,40 \pm 0,03$ & $0,37-0,43$ & $0,36 \pm 0,02$ & $0,34-0,39$ & $0,40 \pm 0,05$ & $0,37-0,43$ & 6,11 \\
\hline Long. Paso/Long. Pierna & $0,77 \pm 0,05$ & $0,73-0,80$ & $0,78 \pm 0,07$ & $0,72-0,84$ & $0,71 \pm 0,07$ & $0,65-0,77$ & $0,76 \pm 0,09$ & $0,70-0,83$ & 5,52 \\
\hline Longitud Zancada $(\mathrm{cm})^{*}$ & $133,39 \pm 6,06$ & $128,73-138,05$ & $141,43 \pm 10,97$ & $131,28-151,57$ & $130,67 \pm 8,93$ & $122,41-138,93$ & $147,70 \pm 17,50$ & $135,18-160,22$ & 8,60 \\
\hline Long. Zanc./Estatura & $0,79 \pm 0,03$ & $0,76-0,81$ & $0,81 \pm 0,06$ & $0,75-0,87$ & $0,73 \pm 0,05$ & $0,68-0,78$ & $0,80 \pm 0,10$ & $0,73-0,87$ & 6,38 \\
\hline Long. Zanc./Long. Pierna & $1,53 \pm 0,09$ & $1,46-1,60$ & $1,56 \pm 0,13$ & $1,44-1,68$ & $1,42 \pm 0,14$ & $1,29-1,54$ & $1,53 \pm 0,19$ & $1,40-1,67$ & 5,47 \\
\hline Anchura Paso $(\mathrm{cm})$ & $13,43 \pm 1,81$ & $12,03-14,82$ & $14,00 \pm 2,24$ & $11,93-16,07$ & $12,09 \pm 2,73$ & $9,57-14,62$ & $12,20 \pm 2,78$ & $10,21-14,19$ & 3,26 \\
\hline \multirow[t]{3}{*}{ Anch. Paso/Anch. Pelvis } & $0,59 \pm 0,08$ & $0,52-0,65$ & $0,59 \pm 0,11$ & $0,49-0,69$ & $0,49 \pm 0,11$ & $0,38-0,59$ & $0,54 \pm 0,14$ & $0,44-0,64$ & 3,50 \\
\hline & \multicolumn{2}{|c|}{ M1 } & \multicolumn{2}{|c|}{ M2 } & \multicolumn{2}{|c|}{ M3 } & \multicolumn{2}{|c|}{ M4 } & \\
\hline & Media \pm DS & IC $95 \%$ & Media \pm DS & IC $95 \%$ & Media \pm DS & IC $95 \%$ & Mean \pm SD & $95 \% \mathrm{Cl}$ & \\
\hline Longitud Paso $(\mathrm{cm})$ & $61,81 \pm 4,13$ & $51,56-72,07$ & $70,23 \pm 5,07$ & $66,00-74,47$ & $68,41 \pm 6,53$ & $63,39-73,42$ & $68,69 \pm 4,45$ & $65,27-72,11$ & 6,07 \\
\hline Long. Paso/Estatura & $0,39 \pm 0,02$ & $0,33-0,45$ & $0,44 \pm 0,03$ & $0,41-0,46$ & $0,41 \pm 0,04$ & $0,38-0,44$ & $0,40 \pm 0,03$ & $0,38-0,43$ & 6,12 \\
\hline Long. Paso/Long. Pierna & $0,75 \pm 0,06$ & $0,62-0,89$ & $0,85 \pm 0,06$ & $0,80-0,90$ & $0,79 \pm 0,08$ & $0,73-0,85$ & $0,78 \pm 0,07$ & $0,72-0,83$ & 6,94 \\
\hline Longitud Zancada $(\mathrm{cm})$ & $124,03 \pm 8,58$ & $102,73-145,33$ & $139,58 \pm 10,61$ & $130,71-148,45$ & $136,57 \pm 12,81$ & $126,73-146,41$ & $137,16 \pm 8,29$ & $130,79-143,53$ & 4,56 \\
\hline Long. Zanc./Estatura & $0,78 \pm 0,05$ & $0,66-0,91$ & $0,87 \pm 0,07$ & $0,81-0,92$ & $0,82 \pm 0,08$ & $0,76-0,89$ & $0,80 \pm 0,06$ & $0,76-0,85$ & 5,29 \\
\hline Long. Zanc./Long. Pierna & $1,51 \pm 0,12$ & $1,23-1,80$ & $1,70 \pm 0,13$ & $1,59-1,80$ & $1,58 \pm 0,15$ & $1,47-1,70$ & $1,56 \pm 0,14$ & $1,45-1,66$ & 6,36 \\
\hline Anchura Paso $(\mathrm{cm})$ & $10,11 \pm 1,85$ & $5,51-14,70$ & $11,13 \pm 1,22$ & $10,11-12,15$ & $11,21 \pm 2,60$ & $9,21-13,21$ & $9,63 \pm 2,09$ & $8,02-11,24$ & 4,58 \\
\hline Anch. Paso/Anch. Pelvis & $0,49 \pm 0,07$ & $0,32-0,66$ & $0,53 \pm 0,08$ & $0,46-0,60$ & $0,51 \pm 0,16$ & $0,39-0,63$ & $0,42 \pm 0,10$ & $0,34-0,49$ & 6,11 \\
\hline
\end{tabular}

Hombres: H1 (<25\%), H2 (25\%-50\%), H3 (50\%-75\%), H4 (>75\%); Mujeres: M1 (<25\%), M2 (25\%-50\%), M3 (50\%-75\%), M4 (>75\%) 
Tabla 8. Parámetros Espacio-temporales: Distancias (Hombres y Mujeres)

\begin{tabular}{lccccc}
\hline & \multicolumn{2}{c}{ Hombres } & \multicolumn{2}{c}{ Mujeres } & Z \\
\cline { 2 - 5 } & Media \pm DS & IC $95 \%$ & Media \pm DS & IC $95 \%$ & F,54 \\
\cline { 2 - 5 } Longitud Paso (cm) & $69,36 \pm 6,58$ & $66,87-71,68$ & $68,32 \pm 5,58$ & $66,20-70,44$ & 1,85 \\
Long. Paso/Estatura + & $0,39 \pm 0,03$ & $0,38-0,40$ & $0,41 \pm 0,04$ & $0,40-0,43$ & $-2,24$ \\
Long. Paso/Long. Pierna + & $0,76 \pm 0,07$ & $0,73-0,78$ & $0,80 \pm 0,07$ & $0,77-0,83$ & $-2,29$ \\
Longitud Zancada (cm) & $138,85 \pm 13,48$ & $133,74-143,58$ & $136,29 \pm 10,92$ & $132,13-140,44$ & $-2,00$ \\
Long. Zancada/Estatura + & $0,78 \pm 0,07$ & $0,76-0,81$ & $0,83 \pm 0,07$ & $0,80-0,85$ & $-2,17$ \\
Long. Zancada/Long. Pierna + & $1,51 \pm 0,15$ & $1,46-1,57$ & $1,60 \pm 0,14$ & $1,54-1,65$ & $-3,42$ \\
Anchura Paso (cm) + & $12,89 \pm 2,44$ & $11,98-13,77$ & $10,58 \pm 2,08$ & $9,79-11,38$ & $-2,29$ \\
Anchura Paso/Anchura Pelvis + & $0,55 \pm 0,12$ & $0,51-0,60$ & $0,49 \pm 0,12$ & $0,44-0,53$ & $+p<0,05$ (diferencias entre hombres y mujeres)
\end{tabular}

\subsubsection{Tiempos}

Ninguna de las variables temporales mostraron diferencias entre los grupos de estatura de los hombres ( $p=0,056$ para porcentaje del ciclo en apoyo $(\%), p=0,056$ para porcentaje del ciclo en oscilación (\%), $p=0,444$ para tiempo de apoyo (s), $p=0,924$ para tiempo de oscilación ( $s$ ), $p=0,573$ para tiempo de paso $(s), p=0,590$ para tiempo de zancada $(s), p=0,691$ para tiempo de apoyo monopodal (s) y $\mathrm{p}=0,232$ para tiempo de doble apoyo (s)) (Tabla 9).

El porcentaje del ciclo en apoyo $(p=0,031)$ mostró diferencias entre los grupos de estatura de las mujeres, siendo los grupos M1 $(p=0,027)$ y $M 4(p=0,008)$ mayores que el grupo M2. El porcentaje del ciclo en oscilación $(p=0,031)$ también presentó diferencias entre las mujeres, mostrando valores mayores en el grupo M2 que en los grupos M1 $(p=0,027)$ y $M 4(p=0,008)$. El tiempo de doble apoyo $(p=0,020)$ mostró valores mayores en los grupos $M 1(p=0,033)$ y M4 $(p=0,003)$ que en el grupo $M 2$. El tiempo de apoyo $(p=0,068)$, el tiempo de oscilación $(p=0,348)$, el tiempo de paso $(p=0,093)$, el tiempo de zancada $(p=0,073)$ y el tiempo de apoyo monopodal $(p=0,087)$ no presentaron diferencias entre los grupos por estatura de las mujeres (Tabla 9). 
Tabla 9. Parámetros Espacio-temporales: Tiempos (Grupos por estatura)

\begin{tabular}{|c|c|c|c|c|c|c|c|c|c|}
\hline & \multicolumn{2}{|c|}{$\mathrm{H} 1$} & \multicolumn{2}{|c|}{$\mathrm{H} 2$} & \multicolumn{2}{|c|}{ H3 } & \multicolumn{2}{|c|}{$\mathrm{H} 4$} & \multirow{2}{*}{$\chi^{2}(3)$} \\
\hline & Media \pm DS & IC $95 \%$ & Media \pm DS & IC $95 \%$ & Media \pm DS & IC $95 \%$ & Media \pm DS & IC $95 \%$ & \\
\hline Porcentaje de Apoyo (\%) & $59,37 \pm 1,58$ & $58,16-60,58$ & $59,25 \pm 0,83$ & $58,48-60,02$ & $59,97 \pm 1,69$ & $58,40-61,53$ & $58,22 \pm 1,33$ & $57,27-59,18$ & 7,56 \\
\hline Porcentaje de Oscilación (\%) & $40,63 \pm 1,58$ & $39,42-41,84$ & $40,75 \pm 0,83$ & $39,98-41,52$ & $40,03 \pm 1,69$ & $38,47-41,60$ & $41,78 \pm 1,33$ & $40,82-42,73$ & 7,56 \\
\hline Tiempo de Apoyo (s) & $0,63 \pm 0,04$ & $0,60-0,66$ & $0,63 \pm 0,05$ & $0,59-0,68$ & $0,67 \pm 0,09$ & $0,59-0,75$ & $0,61 \pm 0,06$ & $0,56-0,66$ & 2,68 \\
\hline Tiempo de Oscilación (s) & $0,43 \pm 0,02$ & $0,42-0,45$ & $0,44 \pm 0,04$ & $0,40-0,47$ & $0,45 \pm 0,03$ & $0,41-0,48$ & $0,44 \pm 0,03$ & $0,41-0,46$ & 0,48 \\
\hline Tiempo de Paso (s) & $0,53 \pm 0,3$ & $0,51-0,55$ & $0,53 \pm 0,04$ & $0,50-0,57$ & $0,56 \pm 0,06$ & $0,50-0,61$ & $0,52 \pm 0,05$ & $0,49-0,55$ & 2,00 \\
\hline Tiempo de Zancada (s) & $1,07 \pm 0,05$ & $1,03-1,10$ & $1,07 \pm 0,08$ & $0,99-1,15$ & $1,12 \pm 0,12$ & $1,01-1,23$ & $1,05 \pm 0,09$ & $0,98-1,11$ & 1,91 \\
\hline Tiempo de Apoyo Monopodal (s) & $0,53 \pm 0,03$ & $0,51-0,55$ & $0,54 \pm 0,04$ & $0,50-0,58$ & $0,56 \pm 0,06$ & $0,50-0,62$ & $0,53 \pm 0,04$ & $0,49-0,56$ & 1,46 \\
\hline \multirow[t]{3}{*}{ Tiempo de Doble Apoyo (s) } & $0,10 \pm 0,02$ & $0,09-0,12$ & $0,10 \pm 0,01$ & $0,09-0,11$ & $0,11 \pm 0,03$ & $0,09-0,14$ & $0,09 \pm 0,02$ & $0,08-0,10$ & 4,29 \\
\hline & \multicolumn{2}{|c|}{ M1 } & \multicolumn{2}{|c|}{ M2 } & \multicolumn{2}{|c|}{ M3 } & \multicolumn{2}{|c|}{ M4 } & \\
\hline & Media \pm DS & IC $95 \%$ & Media \pm DS & IC $95 \%$ & Media \pm DS & IC $95 \%$ & Media \pm DS & IC $95 \%$ & \\
\hline Porcentaje de Apoyo (\%)* & $61,15 \pm 1,28$ & $57,99-64,32$ & $58,97 \pm 0,86$ & $58,25-59,68$ & $60,01 \pm 1,64$ & $58,75-61,27$ & $60,63 \pm 0,85$ & $59,98-61,28$ & 8,84 \\
\hline Porcentaje de Oscilación (\%)* & $38,85 \pm 1,28$ & $35,68-42,01$ & $41,03 \pm 0,86$ & $40,32-41,75$ & $39,99 \pm 1,64$ & $38,73-41,25$ & $39,37 \pm 0,85$ & $38,72-40,02$ & 8,84 \\
\hline Tiempo de Apoyo (s) & $0,66 \pm 0,09$ & $0,43-0,89$ & $0,58 \pm 0,4$ & $0,55-0,61$ & $0,61 \pm 0,06$ & $0,56-0,66$ & $0,65 \pm 0,06$ & $0,61-0,70$ & 7,11 \\
\hline Tiempo de Oscilación (s) & $0,42 \pm 0,04$ & $0,33-0,51$ & $0,40 \pm 0,02$ & $0,38-0,42$ & $0,40 \pm 0,04$ & $0,38-0,43$ & $0,42 \pm 0,03$ & $0,40-0,45$ & 3,30 \\
\hline Tiempo de Paso (s) & $0,54 \pm 0,07$ & $0,38-0,70$ & $0,49 \pm 0,03$ & $0,46-0,51$ & $0,50 \pm 0,05$ & $0,47-0,54$ & $0,54 \pm 0,05$ & $0,50-0,57$ & 6,42 \\
\hline Tiempo de Zancada (s) & $1,08 \pm 0,13$ & $0,76-1,41$ & $0,98 \pm 0,05$ & $0,93-1,02$ & $1,01 \pm 0,10$ & $0,94-1,08$ & $1,08 \pm 0,09$ & $1,01-1,14$ & 6,95 \\
\hline Tiempo de Apoyo Monopodal (s) & $0,54 \pm 0,06$ & $0,38-0,70$ & $0,49 \pm 0,03$ & $0,47-0,51$ & $0,51 \pm 0,05$ & $0,47-0,54$ & $0,54 \pm 0,04$ & $0,50-0,57$ & 6,56 \\
\hline Tiempo de Doble Apoyo (s)* & $0,12 \pm 0,03$ & $0,05-0,19$ & $0,09 \pm 0,01$ & $0,08-0,10$ & $0,10 \pm 0,02$ & $0,09-0,12$ & $0,12 \pm 0,02$ & $0,10-0,13$ & 9,88 \\
\hline
\end{tabular}

Hombres: H1 (<25\%), H2 (25\%-50\%), H3 (50\%-75\%), H4 (>75\%); Mujeres: M1 (<25\%), M2 (25\%-50\%), M3 (50\%-75\%), M4 (>75\%) 
En la comparativa por sexo, el tiempo de oscilación $(p<0,001)$, el tiempo de paso $(p=0,023)$, el tiempo de zancada $(p=0,021)$ y el tiempo de apoyo monopodal $(p=0,023)$ presentaron valores mayores en hombres que en mujeres. Sin embargo, el tiempo de apoyo no presentó diferencias entre sexos $(p=0,121)$. El porcentaje de tiempo de apoyo mostró valores mayores en mujeres en comparación con los hombres únicamente en el grupo por debajo del percentil 25 (H1-M1) $(p=0,046)$ y por encima del percentil 75 de estatura (H4-M4) $(p=0,012)$. Por el contrario, el porcentaje de tiempo de oscilación fue menor en mujeres que en hombres por debajo del percentil 25 (H1-M1) $(p=0,046)$ y por encima del percentil 75 de estatura (H4-M4) $(p=0,012)$. El tiempo de doble apoyo se presentó mayor en mujeres que en hombres por encima del percentil 75 de estatura (H4-M4) $(p=0,010)$ (Tabla 10).

Tabla 10. Parámetros Espacio-temporales: Tiempos (Hombres y mujeres)

\begin{tabular}{|c|c|c|c|c|c|c|}
\hline & \multicolumn{2}{|c|}{ Hombres } & \multicolumn{2}{|c|}{ Mujeres } & \multirow{2}{*}{ Z } & \multirow{2}{*}{$\mathrm{F}_{3,54}$} \\
\hline & Media \pm DS & IC $95 \%$ & Media \pm DS & IC 95\% & & \\
\hline Porcentaje de Apoyo (\%) & $59,13 \pm 1,49$ & $58,60-59,69$ & $60,03 \pm 1,36$ & $59,52-60,55$ & & 3,98 \\
\hline Porcentaje de Oscilación (\%) & $40,87 \pm 1,49$ & $40,31-41,40$ & $39,97 \pm 1,36$ & $39,45-40,48$ & & 3,98 \\
\hline Tiempo de Apoyo (s) & $0,63 \pm 0,06$ & $0,61-0,65$ & $0,62 \pm 0,07$ & $0,59-0,64$ & $-1,17$ & \\
\hline Tiempo de Oscilación (s)† & $0,44 \pm 0,03$ & $0,43-0,44$ & $0,41 \pm 0,03$ & $0,40-0,42$ & $-3,50$ & \\
\hline Tiempo de Paso (s) ${ }^{\dagger}$ & $0,53 \pm 0,04$ & $0,52-0,55$ & $0,51 \pm 0,05$ & $0,50-0,53$ & $-2,00$ & \\
\hline Tiempo de Zancada $(\mathrm{s})^{\dagger}$ & $1,07 \pm 0,09$ & $1,04-1,10$ & $1,03 \pm 0,09$ & $0,99-1,06$ & $-2,03$ & \\
\hline Tiempo de Apoyo Monopodal (s) ${ }^{\dagger}$ & $0,54 \pm 0,4$ & $0,52-0,55$ & $0,52 \pm 0,05$ & $0,50-0,53$ & $-2,00$ & \\
\hline Tiempo de Doble Apoyo (s) & $0,10 \pm 0,02$ & $0,09-0,11$ & $0,11 \pm 0,02$ & $0,10-0,11$ & & 4,15 \\
\hline
\end{tabular}




\subsubsection{Parámetros cinemáticos}

\subsubsection{Pelvis y centro de gravedad}

Entre los grupos de estatura de los hombres no se presentaron diferencias en el rango de movimiento de la pelvis $\left({ }^{\circ}\right)$ en el plano frontal $(p=0,360)$, plano sagital $(p=0,289)$ y plano transversal $(p=0,785)$. De igual forma, tampoco se mostraron diferencias en el movimiento del centro de gravedad $(\mathrm{mm})$ en el plano frontal $(p=0,389)$ y en el plano sagital $(p=0,061)(T a b l a$ 11).

En cuanto el rango de movimiento de la pelvis en el plano sagital (anteversión y retroversión), se detectaron diferencias $(p=0,046)$ entre los diferentes grupos por estaturas en mujeres, siendo mayor la amplitud de movimiento en el grupo M2 que el grupo M1 ( $p=0,005)$. No se mostraron diferencias entre los grupos de estatura de las mujeres en el rango de movimiento de la pelvis en el plano frontal $(p=0,818)$ y el en plano transversal $(p=0,129)$. Así mismo, no se mostraron diferencias en el movimiento del centro de gravedad entre los grupos estatura de las mujeres en el plano frontal $(p=0,507)$ y en el plano sagital $(p=0,935)$ (Tabla 11).

Comparando los parámetros cinemáticos de la pelvis y el centro de gravedad entre hombres y mujeres, se presentaron diferencias en el rango de movimiento de la pelvis en el plano frontal $(p<0,001)$ y en el rango de movimiento del centro de gravedad en el plano frontal $(p<0,001)$. Los hombres mostraron mayor amplitud de movimiento en el desplazamiento lateral del centro de gravedad (plano frontal) que las mujeres, mientras que las mujeres mostraron mayor amplitud de movimiento pélvico en el plano frontal que los hombres. No se mostraron diferencias en el rango de movimiento de la pelvis en planos sagital $(p=0,806)$ y transversal $(p=0,436)$, ni en el rango de movimiento del centro de gravedad en el plano sagital $(p=0,403)$ entre hombres y mujeres (Tabla 12). 
Tabla 11. Parámetros Cinemáticos: Pelvis y Centro de Gravedad (Grupos por estatura)

\begin{tabular}{|c|c|c|c|c|c|c|c|c|c|}
\hline & \multicolumn{2}{|c|}{ H1 } & \multicolumn{2}{|c|}{$\mathrm{H} 2$} & \multicolumn{2}{|c|}{ H3 } & \multicolumn{2}{|c|}{$\mathrm{H} 4$} & \multirow{2}{*}{$x^{2}(3$} \\
\hline & Media \pm DS & IC $95 \%$ & Media \pm DS & IC $95 \%$ & Media \pm DS & IC $95 \%$ & Media \pm DS & IC $95 \%$ & \\
\hline CG plano frontal $(\mathrm{mm})$ & $63,11 \pm 12,45$ & $53,54-72,68$ & $60,06 \pm 11,80$ & $49,18-71,01$ & $67,72 \pm 9,91$ & $58,56-76,88$ & $58,31 \pm 10,44$ & $50,84-65,78$ & 3,02 \\
\hline CG plano sagital $(\mathrm{mm})$ & $41,28 \pm 5,17$ & $37,30-45,25$ & $48,82 \pm 9,08$ & $40,42-57,22$ & $37,95 \pm 5,45$ & $32,92-42,99$ & $47,25 \pm 9,41$ & $40,51-53,98$ & 7,38 \\
\hline Pelvis plano frontal $\left({ }^{\circ}\right)$ & $12,05 \pm 3,41$ & $9,44-14,67$ & $12,43 \pm 3,43$ & $9,25-15,60$ & $9,63 \pm 2,94$ & $6,91-12,34$ & $12,69 \pm 3,47$ & $10,21-15,17$ & 3,21 \\
\hline Pelvis plano sagital $\left({ }^{\circ}\right)$ & $2,79 \pm 0,68$ & $2,26-3,31$ & $3,74 \pm 1,23$ & $2,60-4,88$ & $3,43 \pm 0,84$ & $2,65-4,21$ & $3,44 \pm 0,96$ & $2,76-4,13$ & 3,76 \\
\hline \multirow[t]{3}{*}{ Pelvis plano transversal $\left({ }^{\circ}\right)$} & $9,51 \pm 2,29$ & $7,76-11,27$ & $9,41 \pm 3,97$ & $5,73-13,09$ & $8,58 \pm 1,92$ & $6,80-10,35$ & $9,44 \pm 3,02$ & $7,28-11,60$ & 1,07 \\
\hline & \multicolumn{2}{|c|}{ M1 } & \multicolumn{2}{|c|}{ M2 } & \multicolumn{2}{|c|}{ M3 } & \multicolumn{2}{|c|}{ M4 } & \\
\hline & Media \pm DS & IC $95 \%$ & Media \pm DS & IC $95 \%$ & Media \pm DS & IC $95 \%$ & Media \pm DS & IC $95 \%$ & \\
\hline CG plano frontal $(\mathrm{mm})$ & $56,36 \pm 7,92$ & $36,68-76,04$ & $45,48 \pm 11,92$ & $35,51-55,45$ & $49,25 \pm 8,79$ & $42,49-56,00$ & $49,11 \pm 11,14$ & $40,55-57,67$ & 2,33 \\
\hline CG plano sagital $(\mathrm{mm})$ & $42,86 \pm 13,10$ & $10,32-75,41$ & $44,33 \pm 10,50$ & $35,54-53,11$ & $46,07 \pm 11,41$ & $37,31-54,84$ & $44,44 \pm 9,56$ & $37,09-51,79$ & 0,43 \\
\hline Pelvis plano frontal $\left({ }^{\circ}\right)$ & $13,50 \pm 0,36$ & $12,59-14,40$ & $15,15 \pm 2,56$ & $13,01-17,29$ & $16,19 \pm 4,59$ & $12,65-19,72$ & $15,68 \pm 3,15$ & $13,26-18,11$ & 0,93 \\
\hline Pelvis plano sagital $\left({ }^{\circ}\right)$ * & $2,24 \pm 0,42$ & $1,19-3,29$ & $3,85 \pm 0,78$ & $3,19-4,00$ & $3,57 \pm 1,35$ & $2,53-4,60$ & $3,21 \pm 0,83$ & $2,57-3,85$ & 7,98 \\
\hline Pelvis plano transversal $\left({ }^{\circ}\right)$ & $6,48 \pm 1,59$ & $2,53-10,43$ & $11,11 \pm 3,25$ & $8,39-13,83$ & $9,29 \pm 2,90$ & $7,07-11,52$ & $9,49 \pm 2,78$ & $7,35-11,62$ & 5,67 \\
\hline
\end{tabular}

Hombres: H1 (<25\%), H2 (25\%-50\%), H3 (50\%-75\%), H4 (>75\%); Mujeres: M1 (<25\%), M2 (25\%-50\%), M3 (50\%-75\%), M4 (>75\%)

Tabla 12. Parámetros Cinemáticos: Pelvis y Centro de Gravedad (Hombres y mujeres)

\begin{tabular}{|c|c|c|c|c|c|c|}
\hline & \multicolumn{2}{|c|}{ Hombres } & \multicolumn{2}{|c|}{ Mujeres } & \multirow{2}{*}{ z } & \multirow{2}{*}{$\mathrm{F}_{3,54}$} \\
\hline & Media \pm DS & IC $95 \%$ & Media \pm DS & IC $95 \%$ & & \\
\hline CG plano frontal $(\mathrm{mm})^{\dagger}$ & $61,99 \pm 11,24$ & $57,98-66,21$ & $48,90 \pm 10,34$ & $44,97-52,83$ & $-4,17$ & \\
\hline CG plano sagital (mm) & $43,98 \pm 8,43$ & $40,87-47,04$ & $44,75 \pm 10,21$ & $40,87-44,64$ & $-0,25$ & \\
\hline Pelvis plano frontal $\left({ }^{\circ}\right)^{\dagger}$ & $11,81 \pm 3,39$ & $10,47-12,89$ & $15,47 \pm 3,34$ & $14,20-16,74$ & $-4,13$ & \\
\hline Pelvis plano sagital $\left({ }^{\circ}\right)$ & $3,32 \pm 0,96$ & $2,98-3,68$ & $3,40 \pm 1,05$ & $3,00-3,80$ & & 0,33 \\
\hline Pelvis plano transversal $\left({ }^{\circ}\right)$ & $9,27 \pm 2,77$ & 8,16-10,11 & $9,56 \pm 3,03$ & $8,41-10,71$ & $-0,16$ & \\
\hline
\end{tabular}




\subsubsection{Cadera}

No se mostraron diferencias entre los grupos por estatura de los hombres en los grados de flexión máxima de cadera $(p=0,281)$ y el porcentaje del ciclo en el que sucede la flexión máxima $(0,761)$; en los grados de extensión máxima de cadera $(p=0,641)$ y el porcentaje del ciclo en que se encuentra la extensión máxima $(p=0,056)$; y tampoco en los grados de flexión de cadera en el instante del contacto inicial $(p=0,235)$ (Tabla 13).

Entre los grupos por estatura de las mujeres tampoco se encontraron diferencias en los grados de flexión máxima de cadera $(p=0,963)$ y el porcentaje del ciclo en que sucede la flexión máxima $(0,792)$; en los grados de extensión máxima de cadera $(p=0,848)$ y el porcentaje del ciclo en que se encuentra la extensión máxima $(p=0,054)$; y tampoco en los grados de flexión de cadera en el instante del contacto inicial $(p=0,955)$ (Tabla 13).

Analizando las diferencias entre hombres y mujeres, las mujeres presentaron valores mayores en los grados de flexión máxima de cadera $(p<0,001)$, en los grados de extensión máxima de cadera $(p<0,001)$ y en los grados de flexión de cadera en el contacto inicial $(p<0,001)$. Además, el instante del ciclo (\%) en el que sucede el máximo de flexión de cadera es anterior $(p=0,032)$ en las mujeres que en los hombres. El instante del ciclo (\%) en el que se presenta la máxima extensión de cadera no mostró diferencias entre hombres y mujeres $(p=0,238)$ (Tabla 14). 
Tabla 13. Parámetros Cinemáticos: Cadera (Grupos por estatura)

\begin{tabular}{|c|c|c|c|c|c|c|c|c|c|}
\hline & \multicolumn{2}{|c|}{$\mathrm{H} 1$} & \multicolumn{2}{|c|}{$\mathrm{H} 2$} & \multicolumn{2}{|c|}{$\mathrm{H} 3$} & \multicolumn{2}{|c|}{$\mathrm{H} 4$} & \multirow{2}{*}{$\chi^{2}(3)$} \\
\hline & Media \pm DS & IC $95 \%$ & Media \pm DS & IC $95 \%$ & Media \pm DS & IC $95 \%$ & Media \pm DS & IC $95 \%$ & \\
\hline Flexión Máxima Cadera $\left({ }^{\circ}\right)$ & $43,06 \pm 6,53$ & $38,04-48,07$ & $46,02 \pm 6,80$ & $39,73-52,31$ & $39,44 \pm 4,58$ & $35,20-43,67$ & $43,55 \pm 4,56$ & $40,29-46,82$ & 3,82 \\
\hline Flexión Máxima Cadera (\%) & $87,14 \pm 2,67$ & $85,09-89,19$ & $88,53 \pm 4,02$ & $84,82-92,24$ & $89,91 \pm 5,16$ & $85,14-94,69$ & $87,04 \pm 2,14$ & $85,51-88,58$ & 1,17 \\
\hline Extensión Máxima Cadera $\left({ }^{\circ}\right)$ & $-3,01 \pm 4,30$ & $-6,32-0,3$ & $0,01 \pm 9,56$ & $-8,82-8,85$ & $-3,37 \pm 4,31$ & $-7,36-0,61$ & $-1,21 \pm 5,08$ & $-4,85-2,42$ & 1,68 \\
\hline Extensión Máxima Cadera (\%) & $50,81 \pm 1,33$ & $49,78-51,83$ & $51,62 \pm 0,92$ & $50,77-52,47$ & $51,50 \pm 1,29$ & $50,31-52,70$ & $49,84 \pm 1,46$ & $48,79-50,88$ & 7,57 \\
\hline \multirow[t]{3}{*}{ Flexión Cadera Contacto Inicial $\left({ }^{\circ}\right)$} & $39,53 \pm 4,36$ & $36,18-42,88$ & $42,70 \pm 7,16$ & $36,08-49,32$ & $36,17 \pm 5,32$ & $31,25-41,09$ & $39,75 \pm 4,48$ & $36,54-42,96$ & 4,25 \\
\hline & \multicolumn{2}{|c|}{ M1 } & \multicolumn{2}{|c|}{$\mathrm{M} 2$} & \multicolumn{2}{|c|}{ M3 } & \multicolumn{2}{|c|}{ M4 } & \multirow{2}{*}{$x^{2}(3)$} \\
\hline & Media \pm DS & IC $95 \%$ & Media \pm DS & IC $95 \%$ & Media \pm DS & IC $95 \%$ & Media \pm DS & IC $95 \%$ & \\
\hline Flexión Máxima Cadera ( ${ }^{\circ}$ ) & $50,07 \pm 3,69$ & $40,89-59,25$ & $51,76 \pm 7,38$ & $45,59-57,93$ & $52,38 \pm 7,53$ & $46,59-58,17$ & $50,88 \pm 5,98$ & $46,28-55,47$ & 0,28 \\
\hline Flexión Máxima Cadera (\%) & $87,04 \pm 2,40$ & $81,07-93,02$ & $86,62 \pm 3,76$ & $83,48-89,76$ & $85,42 \pm 6,66$ & $80,30-90,54$ & $85,68 \pm 1,42$ & $84,59-86,77$ & 1,04 \\
\hline Extensión Máxima Cadera $\left({ }^{\circ}\right)$ & $5,63 \pm 2,62$ & $-0,87-12,13$ & $1,36 \pm 10,51$ & $-7,43-10,14$ & $4,06 \pm 6,39$ & $-0,85-8,97$ & $3,73 \pm 4,59$ & $0,20-7,26$ & 0,81 \\
\hline Extensión Máxima Cadera (\%) & $52,42 \pm 0,78$ & $50,47-54,36$ & $50,15 \pm 0,85$ & $49,44-50,86$ & $50,35 \pm 1,08$ & $49,51-51,18$ & $50,98 \pm 1,33$ & $49,96-52,01$ & 7,65 \\
\hline Flexión Cadera Contacto Inicial $\left({ }^{\circ}\right)$ & $48,11 \pm 3,97$ & $38,24-57,98$ & $47,69 \pm 7,24$ & $41,64-53,75$ & $48,39 \pm 7,47$ & $42,65-54,13$ & $46,90 \pm 7,47$ & $41,16-52,65$ & 0,33 \\
\hline
\end{tabular}

Tabla 14. Parámetros Cinemáticos: Cadera (Hombres y Mujeres)

\begin{tabular}{|c|c|c|c|c|c|}
\hline & \multicolumn{2}{|c|}{ Hombres } & \multicolumn{2}{|c|}{ Mujeres } & \multirow{2}{*}{ z } \\
\hline & Media \pm DS & IC $95 \%$ & Media \pm DS & IC $95 \%$ & \\
\hline Flexión Máxima Cadera $\left({ }^{\circ}\right)^{\dagger}$ & $43,07 \pm 5,83$ & $40,83-45,08$ & $51,50 \pm 6,45$ & $49,05-53,96$ & $-4,47$ \\
\hline Flexión Máxima Cadera (\%)† & $87,99 \pm 3,53$ & $86,76-89,34$ & $86,00 \pm 4,19$ & $84,41-87,59$ & $-1,86$ \\
\hline Extensión Máxima Cadera $\left({ }^{\circ}\right)^{\dagger}$ & $-1,90 \pm 5,85$ & $-4,04-0,25$ & $3,37 \pm 6,90$ & $0,75-6,00$ & $-3,46$ \\
\hline Extensión Máxima Cadera (\%) & $50,83 \pm 1,43$ & $50,28-51,33$ & $50,70 \pm 1,24$ & $50,23-51,18$ & $-0,71$ \\
\hline Flexión Cadera Contacto Inicial $\left({ }^{\circ}\right)^{\dagger}$ & $39,56 \pm 5,48$ & $37,59-41,60$ & $47,71 \pm 6,82$ & $45,11-50,30$ & $-4,52$ \\
\hline
\end{tabular}




\subsubsection{Rodilla}

No se mostraron diferencias entre los grupos por estatura de los hombres en los grados de flexión máxima de rodilla $(p=0,943)$ y el porcentaje del ciclo en el que sucede la flexión máxima $(0,348)$; en los grados de extensión máxima de rodilla $(p=0,620)$ y el porcentaje del ciclo en que se encuentra la extensión máxima $(p=0,150)$; y tampoco en los grados de flexión de rodilla en el instante del contacto inicial $(p=0,560)$ (Tabla 15).

Entre los grupos por estatura de las mujeres tampoco se encontraron diferencias en los grados de flexión máxima de rodilla $(p=0,175)$ y el porcentaje del ciclo en que sucede la flexión máxima $(0,159)$; en los grados de extensión máxima de rodilla $(p=0,824)$ y el porcentaje del ciclo en que se encuentra la extensión máxima $(p=0,554)$; y tampoco en los grados de flexión de rodilla en el instante del contacto inicial $(p=0,771)$ (Tabla 15).

En el análisis en función del sexo, las mujeres presentaron valores mayores que los hombres en los grados de máxima flexión de rodilla $(p=0,012)$, en los de máxima extensión de rodilla $(p=0,008)$ y en los de flexión de rodilla en el contacto inicial $(p<0,001)$. Comparando los porcentajes del ciclo de la marcha, las mujeres, además, presentan el máximo de flexión de rodilla después que los hombres $(p=0,013)$. El instante del ciclo (\%) en el que se presenta la máxima extensión de rodilla no mostró diferencias entre hombres y mujeres $(p=0,060)(T a b l a$ 16). 
Tabla 15. Parámetros Cinemáticos: Rodilla (Grupos por estatura)

\begin{tabular}{|c|c|c|c|c|c|c|c|c|c|}
\hline & \multicolumn{2}{|c|}{$\mathrm{H} 1$} & \multicolumn{2}{|c|}{$\mathrm{H} 2$} & \multicolumn{2}{|c|}{ H3 } & \multicolumn{2}{|c|}{$\mathrm{H} 4$} & \multirow{2}{*}{$x^{2}(3)$} \\
\hline & Media \pm DS & IC $95 \%$ & Media \pm DS & IC $95 \%$ & Media \pm DS & IC 95\% & Media \pm DS & IC $95 \%$ & \\
\hline Flexión Máxima Rodilla $\left({ }^{\circ}\right)$ & $65,95 \pm 5,83$ & $61,47-70,44$ & $66,00 \pm 4,59$ & $61,75-70,25$ & $66,14 \pm 3,11$ & $63,26-69,01$ & $66,94 \pm 3,27$ & $64,61-69,28$ & 0,39 \\
\hline Flexión Máxima Rodilla (\%) & $70,80 \pm 1,54$ & $69,62-71,98$ & $70,81 \pm 1,08$ & $69,81-71,81$ & $70,93 \pm 1,27$ & $69,75-72,10$ & $69,87 \pm 1,01$ & $69,15-70,59$ & 3,30 \\
\hline Extensión Máxima Rodilla ( ${ }^{\circ}$ ) & $4,96 \pm 3,80$ & $2,03-7,88$ & $5,56 \pm 5,34$ & $-0,05-11,16$ & $5,90 \pm 4,34$ & $1,89-9,91$ & $6,57 \pm 3,36$ & $4,17-8,98$ & 1,78 \\
\hline Extensión Máxima Rodilla (\%) & $39,71 \pm 1,44$ & $38,6-40,82$ & $38,58 \pm 1,31$ & $37,21-39,96$ & $38,19 \pm 2,65$ & $35,74-40,64$ & $38,08 \pm 1,16$ & $37,25-38,92$ & 5,32 \\
\hline \multirow[t]{3}{*}{ Flexión Rodilla Contacto Inicial $\left({ }^{\circ}\right)$} & $8,11 \pm 3,27$ & $5,60-10,62$ & $7,24 \pm 4,60$ & $2,98-11,50$ & $10,10 \pm 3,63$ & $6,74-13,46$ & $9,27 \pm 3,74$ & $6,60-11,95$ & 2,06 \\
\hline & \multicolumn{2}{|c|}{ M1 } & \multicolumn{2}{|c|}{ M2 } & \multicolumn{2}{|c|}{ M3 } & \multicolumn{2}{|c|}{ M4 } & \\
\hline & Media \pm DS & IC $95 \%$ & Media \pm DS & IC $95 \%$ & Media \pm DS & IC $95 \%$ & Media \pm DS & IC $95 \%$ & \\
\hline Flexión Máxima Rodilla ( ${ }^{\circ}$ ) & $62,82 \pm 2,21$ & $57,33-68,31$ & $69,22 \pm 3,23$ & $66,52-71,91$ & $69,41 \pm 4,63$ & $65,86-72,97$ & $69,60 \pm 4,48$ & $66,16-73,05$ & 4,96 \\
\hline Flexión Máxima Rodilla (\%) & $72,32 \pm 0,44$ & $71,23-73,41$ & $70,69 \pm 1,43$ & $69,49-71,88$ & $70,81 \pm 1,71$ & $69,49-72,12$ & $71,63 \pm 0,95$ & $70,90-72,36$ & 5,18 \\
\hline Extensión Máxima Rodilla ( ${ }^{\circ}$ ) & $8,17 \pm 1,82$ & $3,65-12,69$ & $7,14 \pm 4,13$ & $3,69-10,60$ & $8,57 \pm 2,75$ & $6,45-10,68$ & $8,34 \pm 2,48$ & $6,43-10,24$ & 0,91 \\
\hline Extensión Máxima Rodilla (\%) & $38,12 \pm 2,60$ & $31,65-44,58$ & $37,70 \pm 1,90$ & 36,11 - 39,29 & $37,36 \pm 2,18$ & 35,68 - 39,04 & $38,98 \pm 2,15$ & $37,32-40,63$ & 2,09 \\
\hline Flexión Rodilla Contacto Inicial ( $\left.{ }^{\circ}\right)$ & $10,82 \pm 1,24$ & $7,74-13,91$ & $11,17 \pm 2,48$ & $9,06-13,21$ & $13,37 \pm 4,61$ & $9,83-16,91$ & $12,20 \pm 2,87$ & $9,99-14,41$ & 1,13 \\
\hline
\end{tabular}

Tabla 16. Parámetros Cinemáticos: Rodilla (Hombres y mujeres)

\begin{tabular}{|c|c|c|c|c|c|}
\hline & \multicolumn{2}{|c|}{ Hombres } & \multicolumn{2}{|c|}{ Mujeres } & \multirow{2}{*}{$\mathrm{z}$} \\
\hline & Media \pm DS & IC $95 \%$ & Media \pm DS & IC $95 \%$ & \\
\hline Flexión Máxima Rodilla $\left({ }^{\circ}\right)^{\dagger}$ & $66,30 \pm 4,18$ & $64,68-67,71$ & $68,74 \pm 4,36$ & $67,08-70,39$ & $-2,28$ \\
\hline Flexión Máxima Rodilla (\%)† & $70,55 \pm 1,27$ & $70,11-71,03$ & $71,19 \pm 1,39$ & $70,66-71,71$ & $-2,22$ \\
\hline Extensión Máxima Rodilla $\left({ }^{\circ}\right)^{\dagger}$ & $5,78 \pm 3,96$ & $4,35-7,21$ & $8,06 \pm 2,96$ & $6,94-9,19$ & $-2,41$ \\
\hline Extensión Máxima Rodilla (\%) & $38,66 \pm 1,74$ & $38,03-39,29$ & $38,03 \pm 2,13$ & $37,22-38,84$ & $-1,56$ \\
\hline Flexión Rodilla Contacto Inicial $\left({ }^{\circ}\right) \dagger$ & $8,70 \pm 3,76$ & $7,63-10,22$ & $12,13 \pm 3,32$ & $10,86-13,39$ & $-3,34$ \\
\hline
\end{tabular}


4.1.2.4. Tobillo

Existen diferencias en los grados de máxima extensión de tobillo entre los grupos por estatura de los hombres $(p=0,033)$ ya que el grupo H1 presentó valores inferiores (mayor extensión) que los grupos H4 $(p=0,031)$ y H3 $(p=0,014)$. Por el contrario, los hombres no presentaron diferencias entre los grupos de estatura en los grados de máxima flexión de tobillo $(p=0,462)$ y el porcentaje del ciclo en el que sucede $(p=0,394)$, el porcentaje del ciclo en el que se encuentra la máxima extensión de tobillo $(p=0,208)$ y los grados de flexión de tobillo en el instante del contacto inicial $(p=0,260)$ (Tabla 17).

En cuanto a las mujeres, se detectaron diferencias entre los grupos por estatura en el instante del ciclo en el que sucede la máxima extensión de tobillo $(p=0,018)$ ya que el grupo $M 2$ presentó la máxima extensión del tobillo antes que los grupos M4 ( $p=0,004)$ y $M 1 \quad(p=0,027)$. No se revelaron diferencias entre los grupos por estatura de las mujeres en los grados de máxima flexión de tobillo $(p=0,654)$ y el porcentaje del ciclo en el que sucede $(p=0,730)$, ni en los grados de máxima extensión de tobillo $(p=0,638)$ y los grados de flexión de tobillo en el contacto inicial $(p=0,452)$ (Tabla 17).

Los hombres mostraron valores mayores en los grados de máxima extensión que las mujeres, exceptuando los sujetos situados por debajo del percentil 25 de estatura $\left(F_{3,54}=0,08 ; p=0,780\right.$ para H1-M1; $F_{3,54}=4,56 ; p=0,037$ para $H 2-M_{2} ; F_{3,54}=5,30 ; p=0,025$ para H3-M3; $F_{3,54}=11,19$; $\mathrm{p}=0,001$ para H4-M4). Además, los hombres presentan el instante de máxima extensión posterior a las mujeres teniendo en cuenta el porcentaje del ciclo, exceptuando, también, a los sujetos situados por debajo del percentil 25 de estatura $\left(F_{3,54}=0,00 ; p=0,962\right.$ para H1-M1; $F_{3,54}=9,31 ; p=0,004$ para H2-M2; $F_{3,54}=4,27 ; p=0,044$ para H3-M3; $F_{3,54}=4,29 ; p=0,043$ para H4M4). No se presentaron diferencias entre hombres y mujeres en los grados de flexión máxima de tobillo $(p=0,304)$, el porcentaje del ciclo en el que sucede la máxima flexión de tobillo $(p=0,475)$ y la flexión de tobillo en el contacto inicial $(p=0,096)$ (Tabla 18). 
Tabla 17. Parámetros Cinemáticos: Tobillo (Grupo por estatura)

\begin{tabular}{|c|c|c|c|c|c|c|c|c|c|}
\hline & \multicolumn{2}{|c|}{$\mathrm{H} 1$} & \multicolumn{2}{|c|}{$\mathrm{H} 2$} & \multicolumn{2}{|c|}{ H3 } & \multicolumn{2}{|c|}{$\mathrm{H} 4$} & \multirow{2}{*}{$\chi^{2}(3)$} \\
\hline & Media \pm DS & IC $95 \%$ & Media \pm DS & IC $95 \%$ & Media \pm DS & IC $95 \%$ & Media \pm DS & IC $95 \%$ & \\
\hline Flexión Máxima Tobillo $\left({ }^{\circ}\right)$ & $15,82 \pm 2,05$ & $14,25-17,40$ & $15,92 \pm 3,12$ & $13,04-18,80$ & $16,40 \pm 3,71$ & $12,97-19,84$ & $17,28 \pm 2,21$ & $15,70-18,87$ & 2,58 \\
\hline Extensión Máxima Tobillo $\left({ }^{\circ}\right)^{*}$ & $-15,15 \pm 3,52$ & $-17,85-(-12,45)$ & $-14,90 \pm 5,01$ & $-19,53-(-10,27)$ & $-9,94 \pm 3,26$ & $-12,96-(-6,93)$ & $-10,70 \pm 4,37$ & $-13,83-(-7,57)$ & 8,74 \\
\hline Extensión Máxima Tobillo (\%) & $62,96 \pm 1,40$ & $61,88-64,04$ & $62,87 \pm 1,46$ & $61,53-64,22$ & $63,09 \pm 1,21$ & $61,97-64,21$ & $61,69 \pm 1,36$ & $60,71-62,66$ & 4,55 \\
\hline \multirow{2}{*}{ Flexión Tobillo Contacto Inicial ( $\left.{ }^{\circ}\right)$} & \multicolumn{2}{|c|}{$\mathrm{M} 1$} & \multicolumn{2}{|c|}{$\mathrm{M} 2$} & \multicolumn{2}{|c|}{ M3 } & \multicolumn{2}{|c|}{ M4 } & \\
\hline & Media \pm DS & IC 95\% & Media \pm DS & IC 95\% & Media \pm DS & IC 95\% & Media \pm DS & IC $95 \%$ & \\
\hline Flexión Máxima Tobillo ( ${ }^{\circ}$ ) & $17,37 \pm 3,60$ & $8,42-26,32$ & $15,22 \pm 4,42$ & $11,52-18,91$ & $16,82 \pm 3,36$ & $14,24-19,40$ & $15,47 \pm 2,02$ & $13,91-17,02$ & 1,62 \\
\hline Flexión Máxima Tobillo (\%) & $43,74 \pm 2,55$ & $37,41-50,07$ & $47,35 \pm 20,65$ & $30,09-64,62$ & $43,74 \pm 4,89$ & $39,98-47,49$ & $44,98 \pm 1,74$ & $43,64-46,32$ & 1,30 \\
\hline Extensión Máxima Tobillo $\left({ }^{\circ}\right)$ & $-16,12 \pm 3,56$ & $-24,98-(-7,27)$ & $-20,66 \pm 6,36$ & $-25,98-(-15,34)$ & $-15,99 \pm 8,42$ & $-22,46-(-9,51)$ & $-18,71 \pm 3,59$ & $-21,47-(-15,95)$ & 1,70 \\
\hline
\end{tabular}

Hombres: H1 (<25\%), H2 (25\%-50\%), H3 (50\%-75\%), H4 (>75\%); Mujeres: M1 (<25\%), M2 (25\%-50\%), M3 (50\%-75\%), M4 (>75\%)

Tabla 18. Parámetros Cinemáticos: Tobillo (Hombres y Mujeres)

\begin{tabular}{|c|c|c|c|c|c|c|}
\hline & \multicolumn{2}{|c|}{ Hombres } & \multicolumn{2}{|c|}{ Mujeres } & \multirow{2}{*}{ z } & \multirow{2}{*}{$F_{3,54}$} \\
\hline & Media \pm DS & IC $95 \%$ & Media \pm DS & IC 95\% & & \\
\hline Flexión Máxima Tobillo ( ${ }^{\circ}$ ) & $16,41 \pm 2,69$ & $15,33-17,21$ & $16,02 \pm 3,30$ & $14,76-17,27$ & $-0,51$ & \\
\hline Flexión Máxima Tobillo (\%) & $44,33 \pm 4,64$ & $42,62-46,02$ & $45,12 \pm 10,82$ & $41,00-49,24$ & $-0,06$ & \\
\hline Extensión Máxima Tobillo $\left({ }^{\circ}\right)^{\dagger}$ & $-12,65 \pm 4,56$ & $-14,38-(-11,05)$ & $-18,13 \pm 6,23$ & $-20,50-(-15,77)$ & & 0,93 \\
\hline Extensión Máxima Tobillo (\%) † & $62,58 \pm 1,43$ & $62,07-63,12$ & $61,92 \pm 1,79$ & $61,24-62,60$ & & 5,29 \\
\hline Flexión Tobillo Contacto Inicial ( $\left.{ }^{\circ}\right)$ & $4,94 \pm 2,79$ & $3,98-6,01$ & $4,17 \pm 3,55$ & $2,82-5,52$ & $-1,30$ & \\
\hline
\end{tabular}




\subsection{Comparativa de los patrones de Marcha a velocidad Lenta $(0,25 \mathrm{~m} / \mathrm{s})$ y los patrones de la} Marcha a velocidad Normal

Se analizaron las diferencias en cada una de las variables en función de la velocidad (velocidad normal y velocidad lenta) y en función del grupo de estatura, así como el efecto de la interacción de la velocidad y grupo de estatura en cada una de las variables dependientes. Cada una de las variables se analizó de forma independiente para hombres y mujeres.

\subsubsection{Parámetros espacio-temporales}

\subsubsection{Velocidad y cadencia}

Los hombres presentaron un descenso del $80 \%$ de los valores de velocidad $(p<0,001)$, de velocidad normalizada en función de la estatura $(p<0,001)$ y de velocidad normalizada en función de la longitud de pierna $(p<0,001)$. No se mostraron diferencias en relación al grupo en las mismas variables $(p=0,075$ para velocidad; $p=0,194$ para velocidad normalizada en función de la estatura; $p=0,332$ para velocidad normalizada en función de la longitud de pierna). No hubo efecto de la interacción entre las variables independientes velocidad y grupo en velocidad $(p=0,089)$, velocidad normalizada en función de la estatura $(p=0,206)$ y en velocidad normalizada en función de la longitud de pierna $(p=0,318)$, siendo el comportamiento de todos los grupos de hombres igual en la comparación entre velocidad normal y velocidad lenta. Los valores de la cadencia en hombres disminuyeron alrededor de un $63 \%(p<0,001)$ en relación a la velocidad. No se presentaron diferencias en relación al grupo $(p=0,833)$ y no hubo efecto de la interacción entre velocidad y grupo en la cadencia $(p=0,116)$ (Tabla 19).

Las mujeres, al igual que los hombres, presentaron una disminución del $80 \%$ en la velocidad $(p<0,001)$, la velocidad normalizada en función de la estatura $(p<0,001)$ y la velocidad normalizada en función de la longitud de pierna $(p<0,001)$. No se mostraron diferencias en relación al grupo en las mismas variables $(p=0,233$ para velocidad; $p=0,110$ para velocidad normalizada en función de la estatura; $p=0,076$ para velocidad normalizada en función de la longitud de pierna). Tampoco se encontró efecto de la interacción de velocidad y grupo en velocidad $(p=0,119)$, velocidad normalizada en función de la estatura $(p=0,070)$ y en velocidad normalizada en función de la longitud de pierna $(p=0,060)$. La cadencia presentó un descenso del $65 \%(p<0,001)$ de la velocidad normal a la lenta. No se mostraron diferencias en relación al grupo $(p=0,278)$ y no hubo efecto de la interacción entre velocidad y grupo en la cadencia $(p=0,062)$ (Tabla 19). 
Tabla 19. Parámetros Espacio-temporales: Velocidad y Cadencia (Velocidad Normal y Velocidad Lenta)

\begin{tabular}{|c|c|c|c|c|c|c|c|c|c|c|c|}
\hline & \multicolumn{2}{|c|}{$\mathrm{H} 1$} & \multicolumn{2}{|c|}{$\mathrm{H} 2$} & \multicolumn{2}{|c|}{$\mathrm{H} 3$} & \multicolumn{2}{|c|}{$\mathrm{H} 4$} & \multirow[b]{2}{*}{ Velocidad } & \multirow[b]{2}{*}{ Grupo } & \multirow[b]{2}{*}{ Inter. } \\
\hline & NORMAL & LENTA & NORMAL & LENTA & NORMAL & LENTA & NORMAL & LENTA & & & \\
\hline & Media \pm DS & Media \pm DS & Media \pm DS & Media \pm DS & Media \pm DS & Media \pm DS & Media \pm DS & Media \pm DS & $\mathrm{F}_{1,29}$ & $F_{3,29}$ & $\mathrm{~F}_{3,29}$ \\
\hline Velocidad $(\mathrm{cm} / \mathrm{s})^{*}$ & $125,72 \pm 10,84$ & $25,52 \pm 1,87$ & $132,77 \pm 14,61$ & $27,77 \pm 1,86$ & $118,65 \pm 17,86$ & $25,67 \pm 2,15$ & $143,05 \pm 27,36$ & $25,81 \pm 1,45$ & 920,41 & 2,56 & 2,40 \\
\hline Velocidad/Estatura* & $0,74 \pm 0,06$ & $0,15 \pm 0,01$ & $0,76 \pm 0,08$ & $0,16 \pm 0,01$ & $0,66 \pm 0,10$ & $0,14 \pm 0,01$ & $0,78 \pm 0,15$ & $0,14 \pm 0,01$ & 933,76 & 1,68 & 1,62 \\
\hline Velocidad/Long. Pierna* & $1,44 \pm 0,14$ & $0,29 \pm 0,03$ & $1,46 \pm 0,17$ & $0,31 \pm 0,02$ & $1,29 \pm 0,24$ & $0,28 \pm 0,03$ & $1,49 \pm 0,30$ & $0,27 \pm 0,02$ & 833,55 & 1,19 & 1,23 \\
\hline \multirow[t]{4}{*}{ Cadencia (pasos/min)* } & $113,18 \pm 5,47$ & $43,93 \pm 6,96$ & $113,00 \pm 8,44$ & $40,06 \pm 4,85$ & $108,83 \pm 10,63$ & $43,15 \pm 8,58$ & $116,21 \pm 10,37$ & $40,43 \pm 6,77$ & 2190,93 & 0,29 & 2,15 \\
\hline & \multicolumn{2}{|c|}{ M1 } & \multicolumn{2}{|c|}{ M2 } & \multicolumn{2}{|c|}{ M3 } & \multicolumn{2}{|c|}{ M4 } & & & \\
\hline & NORMAL & LENTA & NORMAL & LENTA & NORMAL & LENTA & NORMAL & LENTA & Velocidad & Grupo & Inter. \\
\hline & Media \pm DS & Media \pm DS & Media \pm DS & Media \pm DS & Media \pm DS & Media \pm DS & Media \pm DS & Media \pm DS & $\mathrm{F}_{1,25}$ & $F_{3,25}$ & $F_{3,25}$ \\
\hline Velocidad $(\mathrm{cm} / \mathrm{s})^{*}$ & $116,29 \pm 21,93$ & $26,65 \pm 1,46$ & $143,48 \pm 17,43$ & $26,08 \pm 1,91$ & $136,88 \pm 23,88$ & $24,70 \pm 2,26$ & $128,53 \pm 14,43$ & $27,04 \pm 1,83$ & 743,01 & 1,52 & 2,15 \\
\hline Velocidad/Estatura* & $0,74 \pm 0,14$ & $0,17 \pm 0,01$ & $0,89 \pm 0,11$ & $0,16 \pm 0,01$ & $0,83 \pm 0,14$ & $0,15 \pm 0,01$ & $0,75 \pm 0,09$ & $0,16 \pm 0,01$ & 731,05 & 2,23 & 2,66 \\
\hline Velocidad/Long. Pierna* & $1,42 \pm 0,28$ & $0,32 \pm 0,01$ & $1,74 \pm 0,21$ & $0,32 \pm 0,02$ & $1,59 \pm 0,27$ & $0,29 \pm 0,03$ & $1,46 \pm 0,19$ & $0,31 \pm 0,02$ & 724,56 & 2,58 & 2,82 \\
\hline Cadencia (pasos $/ \mathrm{min}$ )* & $111,97 \pm 13,68$ & $44,99 \pm 0,62$ & $123,55 \pm 6,99$ & $39,79 \pm 4,60$ & $120,04 \pm 11,26$ & $38,12 \pm 5,42$ & $112,63 \pm 8,94$ & $40,97 \pm 4,74$ & 1005,36 & 1,36 & 2,78 \\
\hline
\end{tabular}

Hombres: H1 (<25\%), H2 (25\%-50\%), H3 (50\%-75\%), H4 (>75\%); Mujeres: M1 (<25\%), M2 (25\%-50\%), M3 (50\%-75\%), M4 (>75\%) 


\subsubsection{Distancias}

La longitud de paso $(p<0,001)$ y la longitud de zancada $(p<0,001)$ en los hombres mostraron una disminución del $45 \%$ en la velocidad lenta comparada con la velocidad normal. Se presentaron diferencias en relación al grupo de estatura en la longitud de paso $(p=0,008)$ y la longitud de zancada $(p=0,006)$. Ambas variables presentaron valores inferiores en los grupos H1 ( $p=0,042$ para longitud de paso; $p=0,032$ para longitud de zancada) y H3 ( $p=0,037$ para longitud de paso; $p=0,035$ para longitud de zancada) que en el grupo H4. No hubo efecto de la interacción entre la velocidad y grupo en la longitud de paso $(p=0,518)$ y la longitud de zancada $(p=0,563)$. La longitud de paso normalizada en función de la estatura $(p<0,001)$ y la longitud de zancada normalizada en función de la estatura $(p<0,001)$ en los hombres mostraron también una disminución del $45 \%$ en la velocidad lenta comparada con la velocidad normal. Existieron diferencias entre los grupos de estatura para la longitud de paso normalizada en función de la estatura $(p=0,042)$ y la longitud de zancada normalizada en función de la estatura $(p=0,040)$. Las dos variables presentaron valores mayores en el grupo H2 $(p=0,033$ para longitud de paso normalizada en función de la estatura; $p=0,033$ para longitud de zancada normalizada en función de la estatura) que en el grupo H3. No hubo efecto de la interacción entre la velocidad y grupo en la longitud de paso normalizada en función de la estatura $(p=0,649)$ y la longitud de zancada normalizada en función de la estatura $(p=0,700)$. La longitud de paso normalizada en función de la longitud de pierna $(p<0,001)$ y la longitud de zancada normalizada en función de la longitud de pierna $(p<0,001)$ en los hombres, mostraron también una disminución del $45 \%$ en la velocidad lenta comparada con la velocidad normal. No se mostraron diferencias comparando los grupos de estatura ( $p=0,099$ para la longitud de paso normalizada en función de la longitud de pierna; $p=0,102$ para la longitud de zancada normalizada en función de la longitud de pierna), ni se mostró efecto de la interacción entre la velocidad y el grupo en la longitud de paso normalizada en función de la longitud de pierna $(p=0,708)$ y en la longitud de zancada normalizada en función de la longitud de pierna $(p=0,757)$. La anchura de paso $(p=0,026)$ y la anchura de paso normalizada $(p=0,028)$ aumentaron en los hombres un $9 \%$. Ninguna de las dos variables presentó diferencias en la comparación de grupos ( $p=0,560$ para anchura de paso; $p=0,368$ para la anchura de paso normalizada), ni se mostró efecto de la interacción de la velocidad y grupo $(p=0,714$ para anchura de paso; $p=0,676$ para la anchura de paso normalizada) (Tabla 20). 
Tabla 20. Parámetros Espacio-temporales: Distancias (Velocidad Normal y Velocidad Lenta)

\begin{tabular}{|c|c|c|c|c|c|c|c|c|c|c|c|}
\hline & \multicolumn{2}{|c|}{$\mathrm{H} 1$} & \multicolumn{2}{|c|}{$\mathrm{H} 2$} & \multicolumn{2}{|c|}{ H3 } & \multicolumn{2}{|c|}{$\mathrm{H} 4$} & \multirow[b]{2}{*}{ Velocidad } & \multirow[b]{2}{*}{ Grupo } & \multirow[b]{2}{*}{ Inter. } \\
\hline & NORMAL & LENTA & NORMAL & LENTA & NORMAL & LENTA & NORMAL & LENTA & & & \\
\hline & Media \pm DS & Media \pm DS & Media \pm DS & Media \pm DS & Media \pm DS & Media \pm DS & Media \pm DS & Media \pm DS & $\mathrm{F}_{1,29}$ & $\mathrm{~F}_{3,29}$ & $F_{3,29}$ \\
\hline Longitud Paso $(\mathrm{cm})^{* \dagger}$ & $66,89 \pm 3,14$ & $35,51 \pm 5,07$ & $70,41 \pm 5,44$ & $41,39 \pm 4,07$ & $65,28 \pm 4,20$ & $36,18 \pm 6,20$ & $73,71 \pm 8,52$ & $38,92 \pm 6,80$ & 410,10 & 4,83 & 0,77 \\
\hline Long. Paso/Estatura*† & $0,39 \pm 0,02$ & $0,21 \pm 0,03$ & $0,40 \pm 0,03$ & $0,24 \pm 0,02$ & $0,36 \pm 0,02$ & $0,20 \pm 0,03$ & $0,40 \pm 0,05$ & $0,21 \pm 0,04$ & 422,75 & 3,11 & 0,56 \\
\hline Long. Paso/Long. Pierna* & $0,77 \pm 0,05$ & $0,41 \pm 0,06$ & $0,78 \pm 0,07$ & $0,46 \pm 0,04$ & $0,71 \pm 0,07$ & $0,39 \pm 0,06$ & $0,76 \pm 0,09$ & $0,40 \pm 0,07$ & 403,34 & 2,29 & 0,47 \\
\hline Longitud Zancada $(\mathrm{cm})^{*+}$ & $133,39 \pm 6,06$ & $71,67 \pm 10,44$ & $141,43 \pm 10,97$ & $83,30 \pm 7,65$ & $130,67 \pm 8,93$ & $73,12 \pm 11,90$ & $147,70 \pm 17,50$ & $78,97 \pm 13,99$ & 393,34 & 5,03 & 0,69 \\
\hline Long. Zancada/Estatura*† & $0,79 \pm 0,03$ & $0,42 \pm 0,06$ & $0,81 \pm 0,06$ & $0,48 \pm 0,04$ & $0,73 \pm 0,05$ & $0,41 \pm 0,07$ & $0,80 \pm 0,10$ & $0,43 \pm 0,07$ & 406,00 & 3,24 & 0,48 \\
\hline Long. Zancada/Long. Pierna* & $1,53 \pm 0,09$ & $0,82 \pm 0,13$ & $1,56 \pm 0,13$ & $0,92 \pm 0,08$ & $1,42 \pm 0,14$ & $0,79 \pm 0,12$ & $1,53 \pm 0,19$ & $0,82 \pm 0,14$ & 388,07 & 2,27 & 0,40 \\
\hline Anchura Paso $(\mathrm{cm})^{*}$ & $13,43 \pm 1,81$ & $13,93 \pm 3,26$ & $14,00 \pm 2,24$ & $14,57 \pm 1,95$ & $12,09 \pm 2,73$ & $13,47 \pm 2,29$ & $12,20 \pm 2,78$ & $13,57 \pm 2,52$ & 5,52 & 0,70 & 0,46 \\
\hline \multirow[t]{4}{*}{ Anchura Paso/Anchura Pelvis* } & $0,59 \pm 0,08$ & $0,61 \pm 0,13$ & $0,59 \pm 0,11$ & $0,62 \pm 0,10$ & $0,49 \pm 0,11$ & $0,54 \pm 0,07$ & $0,54 \pm 0,14$ & $0,60 \pm 0,14$ & 5,32 & 1,09 & 0,51 \\
\hline & \multicolumn{2}{|c|}{ M1 } & \multicolumn{2}{|c|}{ M2 } & \multicolumn{2}{|c|}{ M3 } & \multicolumn{2}{|c|}{ M4 } & & & \\
\hline & NORMAL & LENTA & NORMAL & LENTA & NORMAL & LENTA & NORMAL & LENTA & Velocidad & Grupo & Inter. \\
\hline & Media \pm DS & Media \pm DS & Media \pm DS & Media \pm DS & Media \pm DS & Media \pm DS & Media \pm DS & Media \pm DS & $\mathrm{F}_{1,25}$ & $F_{3,25}$ & $\mathrm{~F}_{3,25}$ \\
\hline Longitud Paso $(\mathrm{cm})^{*}$ & $61,81 \pm 4,13$ & $35,31 \pm 1,88$ & $70,23 \pm 5,07$ & $39,19 \pm 4,02$ & $68,41 \pm 6,53$ & $39,93 \pm 6,04$ & $68,69 \pm 4,45$ & $39,43 \pm 3,74$ & 726,04 & 1,68 & 0,67 \\
\hline Long. Paso/Estatura* & $0,39 \pm 0,02$ & $0,22 \pm 0,01$ & $0,44 \pm 0,03$ & $0,24 \pm 0,02$ & $0,41 \pm 0,04$ & $0,24 \pm 0,04$ & $0,40 \pm 0,03$ & $0,23 \pm 0,02$ & 700,42 & 1,66 & 0,90 \\
\hline Long. Paso/Long. Pierna* & $0,75 \pm 0,06$ & $0,43 \pm 0,02$ & $0,85 \pm 0,06$ & $0,48 \pm 0,04$ & $0,79 \pm 0,08$ & $0,46 \pm 0,07$ & $0,78 \pm 0,07$ & $0,45 \pm 0,05$ & 642,53 & 2,08 & 1,04 \\
\hline Longitud Zancada $(\mathrm{cm})^{*}$ & $124,03 \pm 8,58$ & $70,75 \pm 3,68$ & $139,58 \pm 10,61$ & $78,92 \pm 8,24$ & $136,57 \pm 12,81$ & $79,34 \pm 11,67$ & $137,16 \pm 8,29$ & $79,46 \pm 7,35$ & 624,16 & 1,72 & 0,35 \\
\hline Long. Zancada/Estatura* & $0,78 \pm 0,05$ & $0,45 \pm 0,02$ & $0,87 \pm 0,07$ & $0,49 \pm 0,05$ & $0,82 \pm 0,08$ & $0,48 \pm 0,07$ & $0,80 \pm 0,06$ & $0,46 \pm 0,05$ & 604,44 & 1,66 & 0,55 \\
\hline Long. Zancada/Long. Pierna* & $1,51 \pm 0,12$ & $0,86 \pm 0,04$ & $1,70 \pm 0,13$ & $0,96 \pm 0,09$ & $1,58 \pm 0,15$ & $0,92 \pm 0,13$ & $1,56 \pm 0,14$ & $0,90 \pm 0,09$ & 563,80 & 2,12 & 0,66 \\
\hline Anchura Paso $(\mathrm{cm})^{*+}$ & $10,11 \pm 1,85$ & $11,06 \pm 2,79$ & $11,13 \pm 1,22$ & $13,02 \pm 1,55$ & $11,21 \pm 2,60$ & $13,23 \pm 2,10$ & $9,63 \pm 2,09$ & $9,90 \pm 2,25$ & 12,95 & 3,30 & 1,95 \\
\hline Anchura Paso/Anchura Pelvis*† & $0,49 \pm 0,07$ & $0,54 \pm 0,12$ & $0,53 \pm 0,08$ & $0,62 \pm 0,07$ & $0,51 \pm 0,16$ & $0,60 \pm 0,12$ & $0,42 \pm 0,10$ & $0,43 \pm 0,10$ & 12,38 & 3,91 & 1,86 \\
\hline
\end{tabular}


Las mujeres mostraron una disminución del $42 \%$ en la velocidad lenta comparada con la velocidad normal, en los valores de la longitud de paso $(p=0<0,001)$, la longitud de paso normalizada en función de la estatura $(p<0,001)$, la longitud de paso normalizada en función de la longitud de pierna $(p<0,001)$, la longitud de zancada $(p<0,001)$, la longitud de zancada normalizada en función de la estatura $(p<0,001)$ y la longitud de zancada normalizada en función de la longitud de pierna $(p<0,001)$. Ninguna de las variables presentó diferencias entre los grupos de estatura ( $p=0,198$ para la longitud de paso; $p=0,200$ para la longitud de paso normalizada en función de la estatura; $p=0,128$ para la longitud de paso normalizada en función de la longitud de pierna; $p=0,189$ para la longitud de zancada; $p=0,202$ para la longitud de zancada normalizada en función de la estatura; $p=0,123$ para la longitud de zancada normalizada en función de la longitud de pierna). Tampoco se mostró, en ninguna de las variables nombradas, en las mujeres, efecto de la interacción de velocidad y grupo $(p=0,580$ para la longitud de paso; $p=0,454$ para la longitud de paso normalizada en función de la estatura; $p=0,391$ para la longitud de paso normalizada en función de la longitud de pierna; $p=0,789$ para la longitud de zancada; $p=0,653$ para la longitud de zancada normalizada en función de la estatura; $p=0,587$ para la longitud de zancada normalizada en función de la longitud de pierna). La anchura de paso $(p=0,001)$ y la anchura de paso normalizada $(p=0,002)$ presentaron un aumento del $13,5 \%$ en la velocidad lenta en las mujeres. La anchura de paso mostró diferencias entre los grupos de estatura $(p=0,037)$, siendo los grupos centrales (M2 y M3) mayores que los grupos extremos (M1 y M4) (Figura 41). No se presentó efecto de la interacción de velocidad y grupo en la anchura de paso de las mujeres $(p=0,147)$. La anchura de paso normalizada mostró diferencias entre los grupos de estatura $(p=0,020)$, siendo los valores de M2 mayores que de M4 $(p=0,032)$. No se presentó efecto de la interacción de velocidad y grupo en la anchura de paso normalizada en mujeres $(p=0,163)$ (Tabla 20). 


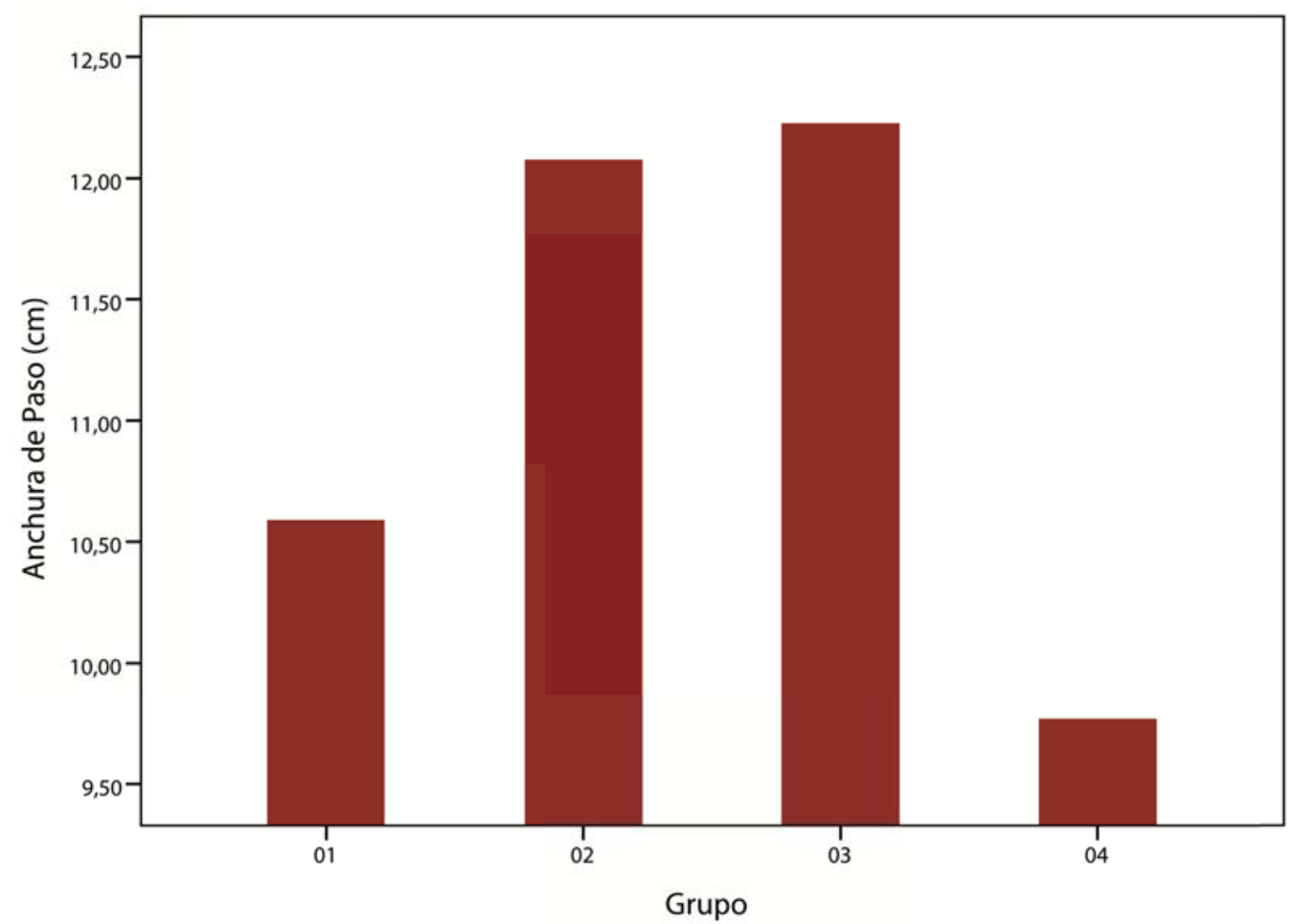

Figura 41. Ancho de paso en mujeres (Grupos de estatura)

\subsubsection{Tiempos}

El tiempo de paso $(p<0,001)$ y el tiempo de zancada (ciclo) $(p<0,001)$ en los hombres se triplicó aproximadamente en la velocidad lenta. No se mostraron diferencias en las comparaciones entre grupos ( $p=0,783$ para el tiempo de paso; $p=0,821$ para el tiempo de zancada) y no se presentó efecto de la interacción de velocidad y grupo de estatura en ell tiempo de paso $(p=0,502)$ y el tiempo de zancada $(p=0,541)$. Los hombres presentaron un aumento del porcentaje de apoyo del $13,5 \%(p<0,001)$ y una consecuente disminución del porcentaje de oscilación $(p<0,001)$ en la velocidad lenta. No se encontraron diferencias entre los grupos de estatura de los hombres en el porcentaje de apoyo $(p=0,454)$ y el porcentaje de oscilación $(p=0,454)$. El efecto de la interacción entre la velocidad y grupo mostró en el porcentaje de apoyo $(p=0,002)$ un aumento mayor en la velocidad lenta en el grupo por encima del percentil 75 (H4), y también, en el porcentaje de oscilación $(p=0,002)$ una disminución mayor en la velocidad lenta en el mismo grupo (H4) (Figuras 42 y 43 ) (Tabla 21). 


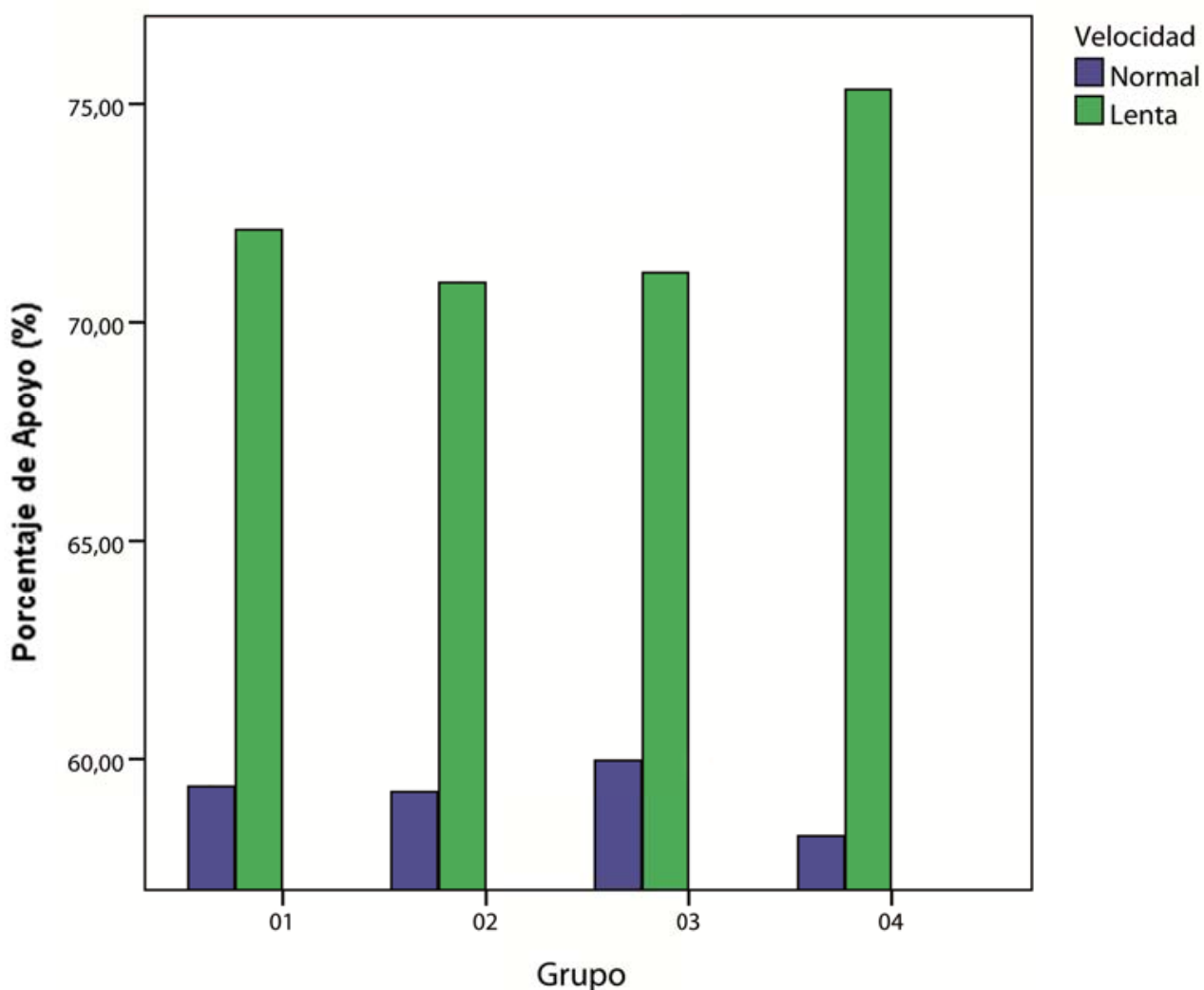

Figura 42. Porcentaje Apoyo en hombres (Velocidad Normal y Velocidad Lenta)

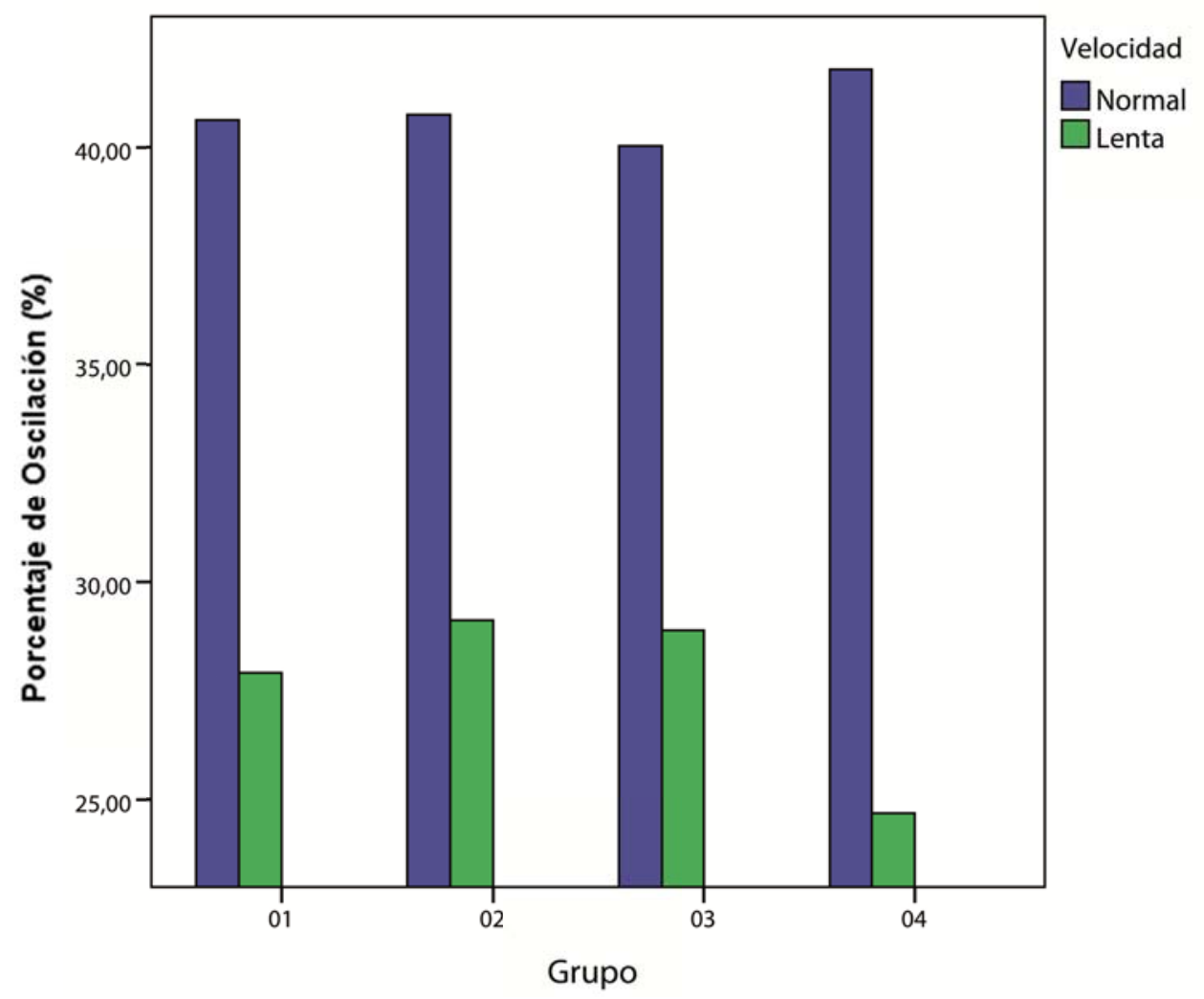

Figura 43. Porcentaje Oscilación en hombres (Velocidad Normal y Velocidad Lenta) 
Tabla 21. Parámetros Espacio-temporales: Tiempos (Velocidad Normal y Velocidad Lenta)

\begin{tabular}{|c|c|c|c|c|c|c|c|c|c|c|c|}
\hline & \multicolumn{2}{|c|}{$\mathrm{H} 1$} & \multicolumn{2}{|c|}{$\mathrm{H} 2$} & \multicolumn{2}{|c|}{ H3 } & \multicolumn{2}{|c|}{$\mathrm{H} 4$} & \multirow[b]{2}{*}{ Velocidad } & \multirow[b]{2}{*}{ Grupo } & \multirow[b]{2}{*}{ Inter. } \\
\hline & NORMAL & LENTA & NORMAL & LENTA & NORMAL & LENTA & NORMAL & LENTA & & & \\
\hline & Media \pm DS & Media \pm DS & Media \pm DS & Media \pm DS & Media \pm DS & Media \pm DS & Media \pm DS & Media \pm DS & $\mathrm{F}_{1,29}$ & $F_{3,29}$ & $\mathrm{~F}_{3,29}$ \\
\hline Tiempo de Paso (s)* & $0,53 \pm 0,3$ & $1,41 \pm 0,24$ & $0,53 \pm 0,04$ & $1,53 \pm 0,21$ & $0,56 \pm 0,06$ & $1,44 \pm 0,26$ & $0,52 \pm 0,05$ & $1,53 \pm 0,26$ & 554,88 & 0,36 & 0,80 \\
\hline Tiempo de Zancada (Ciclo) (s)* & $1,07 \pm 0,05$ & $2,84 \pm 0,50$ & $1,07 \pm 0,08$ & $3,03 \pm 0,42$ & $1,12 \pm 0,12$ & $2,87 \pm 0,52$ & $1,05 \pm 0,09$ & $3,08 \pm 0,54$ & 533,33 & 0,31 & 0,73 \\
\hline Porcentaje de Aроуо (\%)* & $59,37 \pm 1,58$ & $72,10 \pm 4,36$ & $59,25 \pm 0,083$ & $70,76 \pm 3,09$ & $59,97 \pm 1,69$ & $71,12 \pm 3,39$ & $58,22 \pm 1,33$ & $75,31 \pm 3,45$ & 556,80 & 0,90 & 6,57 \\
\hline Porcentaje de Oscilación $(\%)^{*} \ddagger$ & $40,63 \pm 1,58$ & $27,90 \pm 4,36$ & $40,75 \pm 0,83$ & $29,24 \pm 3,09$ & $40,03 \pm 1,69$ & $28,88 \pm 3,39$ & $41,78 \pm 1,33$ & $24,69 \pm 3,45$ & 556,80 & 0,90 & 6,57 \\
\hline Tiempo de Apoyo (s)* & $0,63 \pm 0,04$ & $2,05 \pm 0,42$ & $0,63 \pm 0,05$ & $2,15 \pm 0,30$ & $0,67 \pm 0,09$ & $2,04 \pm 0,40$ & $0,61 \pm 0,06$ & $2,33 \pm 0,46$ & 517,09 & 0,62 & 1,46 \\
\hline Tiempo de Oscilación (s)* & $0,43 \pm 0,02$ & $0,79 \pm 0,16$ & $0,44 \pm 0,04$ & $0,89 \pm 0,16$ & $0,45 \pm 0,03$ & $0,83 \pm 0,17$ & $0,44 \pm 0,03$ & $0,75 \pm 0,15$ & 206,60 & 0,96 & 1,15 \\
\hline Tiempo de Apoyo Monopodal (s)* & $0,53 \pm 0,03$ & $1,43 \pm 0,26$ & $0,54 \pm 0,04$ & $1,51 \pm 0,21$ & $0,56 \pm 0,06$ & $1,43 \pm 0,26$ & $0,53 \pm 0,04$ & $1,54 \pm 0,28$ & 507,43 & 0,27 & 0,68 \\
\hline \multirow[t]{4}{*}{ Tiempo de Doble Apoyo $(s)^{*}$} & $0,10 \pm 0,02$ & $0,64 \pm 0,19$ & $0,10 \pm 0,01$ & $0,62 \pm 0,10$ & $0,11 \pm 0,03$ & $0,60 \pm 0,16$ & $0,09 \pm 0,02$ & $0,79 \pm 0,21$ & 358,66 & 1,57 & 2,82 \\
\hline & \multicolumn{2}{|c|}{ M1 } & \multicolumn{2}{|c|}{ M2 } & \multicolumn{2}{|c|}{ M3 } & \multicolumn{2}{|c|}{ M4 } & & & \\
\hline & NORMAL & LENTA & NORMAL & LENTA & NORMAL & LENTA & NORMAL & LENTA & Velocidad & Grupo & Inter. \\
\hline & Media \pm DS & Media \pm DS & Media \pm DS & Media \pm DS & Media \pm DS & Media \pm DS & Media \pm DS & Media \pm DS & $\mathrm{F}_{1,25}$ & $F_{3,25}$ & $\mathrm{~F}_{3,25}$ \\
\hline Tiempo de Paso (s)* & $0,54 \pm 0,07$ & $1,34 \pm 0,02$ & $0,49 \pm 0,03$ & $1,54 \pm 0,16$ & $0,50 \pm 0,05$ & $1,61 \pm 0,24$ & $0,54 \pm 0,05$ & $1,48 \pm 0,14$ & 589,98 & 1,40 & 2,34 \\
\hline Tiempo de Zancada (Ciclo) (s)* & $1,08 \pm 0,13$ & $2,67 \pm 0,05$ & $0,98 \pm 0,05$ & $3,05 \pm 0,33$ & $1,01 \pm 0,10$ & $3,23 \pm 0,47$ & $1,08 \pm 0,09$ & $2,96 \pm 0,28$ & 610,30 & 1,68 & 2,58 \\
\hline Porcentaje de Apoyo (\%)* & $61,15 \pm 1,28$ & $72,73 \pm 6,74$ & $58,97 \pm 0,86$ & $74,02 \pm 1,74$ & $60,01 \pm 1,64$ & $75,72 \pm 3,35$ & $60,63 \pm 0,85$ & $75,39 \pm 1,79$ & 556,62 & 1,33 & 1,51 \\
\hline Porcentaje de Oscilación (\%)* & $38,85 \pm 1,28$ & $27,27 \pm 6,74$ & $41,03 \pm 0,86$ & $25,98 \pm 1,74$ & $39,99 \pm 1,64$ & $24,28 \pm 3,35$ & $39,37 \pm 0,85$ & $24,61 \pm 1,79$ & 556,62 & 1,33 & 1,51 \\
\hline Tiempo de Apoyo (s)* & $0,66 \pm 0,09$ & $1,94 \pm 0,19$ & $0,58 \pm 0,4$ & $2,26 \pm 0,26$ & $0,61 \pm 0,06$ & $2,45 \pm 0,43$ & $0,65 \pm 0,06$ & $2,23 \pm 0,22$ & 580,25 & 1,84 & 2,64 \\
\hline Tiempo de Oscilación (s)* & $0,42 \pm 0,04$ & $0,73 \pm 0,18$ & $0,40 \pm 0,02$ & $0,79 \pm 0,10$ & $0,40 \pm 0,04$ & $0,78 \pm 0,11$ & $0,42 \pm 0,03$ & $0,73 \pm 0,08$ & 202,71 & 0,33 & 1,05 \\
\hline Tiempo de Apoyo Monopodal (s)* & $0,54 \pm 0,06$ & $1,33 \pm 0,04$ & $0,49 \pm 0,03$ & $1,51 \pm 0,16$ & $0,51 \pm 0,05$ & $1,62 \pm 0,22$ & $0,54 \pm 0,04$ & $1,47 \pm 0,14$ & 621,35 & 1,99 & 2,82 \\
\hline Tiempo de Doble Apoyo (s)* & $0,12 \pm 0,03$ & $0,60 \pm 0,17$ & $0,09 \pm 0,01$ & $0,72 \pm 0,10$ & $0,10 \pm 0,02$ & $0,84 \pm 0,20$ & $0,12 \pm 0,02$ & $0,75 \pm 0,09$ & 443,82 & 2,15 & 2,70 \\
\hline
\end{tabular}


El tiempo de apoyo en los hombres aumentó tres veces y media en la velocidad lenta $(p<0,001)$ y el tiempo de oscilación aumento un $84 \%(p<0,001)$, casi duplicándose, con respecto a la velocidad normal. Ninguna de las dos variables presentaron diferencias entre los grupos ( $p=0,609$ para el tiempo de apoyo; $p=0,425$ para el tiempo de oscilación). No se mostró efecto de la interacción entre velocidad y grupo en el tiempo de apoyo $(p=0,247)$ y el tiempo de oscilación $(p=0,347)$. El tiempo de apoyo monopodal en la velocidad lenta fue aproximadamente tres veces mayor $(p<0,001)$ que en la velocidad normal, y el tiempo de doble apoyo en velocidad lenta septuplicó los valores en velocidad normal $(p<0,001)$. No se presentaron diferencias entre los grupos en el tiempo de apoyo monopodal $(p=0,847)$ y el tiempo de doble apoyo $(p=0,571)$. Tampoco hubo efecto de la interacción de velocidad y grupo en el tiempo de apoyo monopodal $(p=0,571)$ y el tiempo de doble apoyo $(p=0,056)$ (Tabla 21$)$. Las mujeres mostraron en la velocidad lenta los valores de los tiempos de paso $(p<0,001)$ y de zancada (ciclo) $(p<0,001)$ triplicados en comparación con la velocidad normal. No mostraron diferencias en la comparación de los grupos de estatura $(p=0,267$ para el tiempo de paso; $p=0,197$ para el tiempo de zancada), y tampoco se mostró efecto de la interacción de la velocidad y el grupo de estatura en el tiempo de paso $(p=0,098)$ y el tiempo de zancada $(p=0,076)$. El porcentaje de apoyo en las mujeres aumentó un $15 \%(p<0,001)$ y el porcentaje de oscilación disminuyó en consecuencia $(p<0,001)$. No presentaron diferencias en la comparación por grupos ( $p=0,286$ para el porcentaje de apoyo; $p=0,286$ para el porcentaje de oscilación). No se mostró, tampoco, efecto de la interacción de velocidad y grupo de estatura en el porcentaje de apoyo $(p=0,235)$ y el porcentaje de oscilación $(p=0,235)$. El tiempo de apoyo prácticamente se cuadriplicó $(p<0,001)$ en la velocidad lenta, mientras que el tiempo de oscilación aproximadamente se duplicó $(p<0,001)$. Ninguna de las dos variable presentó diferencias en las comparaciones por grupos ( $p=0,166$ para el tiempo de apoyo; $p=0,804$ para el tiempo de oscilación), ni mostró efecto de la interacción entre velocidad y grupo de estatura ( $p=0,072$ para el tiempo de apoyo; $p=0,390$ para el tiempo de oscilación). El tiempo de apoyo monopodal en las mujeres se triplicó $(p<0,001)$ y el tiempo de doble apoyo septuplicó sus valores $(p<0,001)$ en la velocidad lenta comparando con la velocidad normal. No se presentaron diferencias entre los grupos de estatura $(p=0,142$ para el tiempo de apoyo monopodal; $p=0,120$ para el tiempo de doble apoyo), y no se mostró efecto de la interacción entre la velocidad y el grupo de estatura en el tiempo de apoyo monopodal $(p=0,059)$ y el tiempo de doble apoyo $(p=0,067)$ (Tabla 21). 


\subsubsection{Parámetros cinemáticos}

\subsubsection{Pelvis y centro de gravedad}

El movimiento del centro de gravedad en el plano frontal en los hombres aumentó dos veces y media $(p<0,001)$ en la velocidad lenta en relación a la velocidad normal. No presentó diferencias en las comparación entre grupos $(p=0,561)$ y no se mostró efecto de la interacción entre velocidad y grupo de estatura en esta variable $(p=0,698)$. En el plano sagital, el movimiento del centro de gravedad se redujo a más de la mitad (disminuyó un 60\%) en la velocidad lenta $(p<0,001)$. En las comparaciones por grupos se encontraron diferencias $(p=0,005)$, mostrando valores menores en el movimiento del centro de gravedad en el plano sagital para el grupo H3 que para los grupos H2 $(p=0,021)$ y H4 $(p=0,045)$ (Figura 44). No hubo efecto de la interacción entre velocidad y grupo de estatura en esta variable $(p=0,363)$ (Tabla 22).

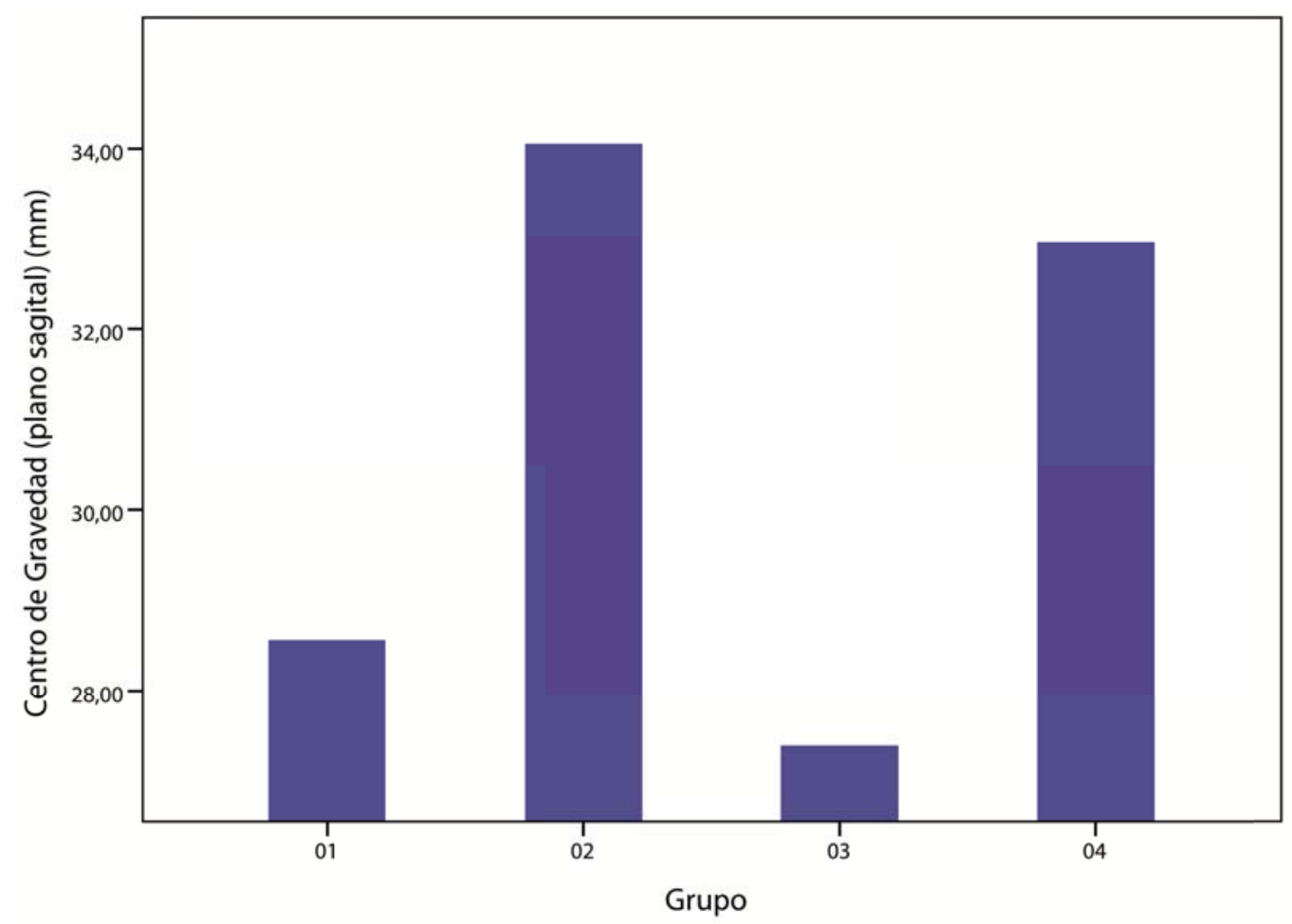

Figura 44. Centro de Gravedad (plano sagital) en hombres (Grupos de estatura)

Los hombres mostraron una disminución del $26 \%$ en el movimiento de la pelvis en el plano frontal $(p<0,001)$ y un aumento del $26 \%$ en el movimiento de la pelvis en el plano sagital $(p=0,020)$. Ninguna de las dos variables presentó diferencias en las comparaciones por grupos ( $p=0,413$ para el movimiento de la pelvis en el plano frontal; $p=0,138$ para el movimiento de la pelvis en el plano sagital), y tampoco se mostró efecto de la interacción entre velocidad y grupo de estatura en el movimiento de la pelvis en el plano frontal $(p=0,289)$ y en el 
movimiento de la pelvis en el plano sagital $(p=0,580)$. No existieron diferencias en los hombres en el movimiento de la pelvis en el plano transversal entre las velocidades normal y lenta $(p=0,086)$, ni en las comparaciones entre grupos $(p=0,755)$. Tampoco hubo efecto de la interacción entre la velocidad y el grupo de estatura en el movimiento de la pelvis en el plano transversal $(p=0,538)$ (Tabla 22).

Las mujeres, al igual que los hombres, presentaron un aumento del $150 \%$ del movimiento del centro de gravedad en el plano frontal $(p<0,001)$ en la velocidad lenta en comparación con la normal. No se mostraron diferencias en las comparaciones por grupos $(p=0,239)$, pero, en cambio, existió efecto en la interacción entre velocidad y grupo de estatura $(p=0.001)$, mostrando valores mayores en el movimiento del centro de gravedad en el plano frontal en el grupo M3 que en M4 $(p=0,024)$. Dichas diferencias fueron detectadas únicamente durante la velocidad lenta (Figura 45) (Tabla 22).

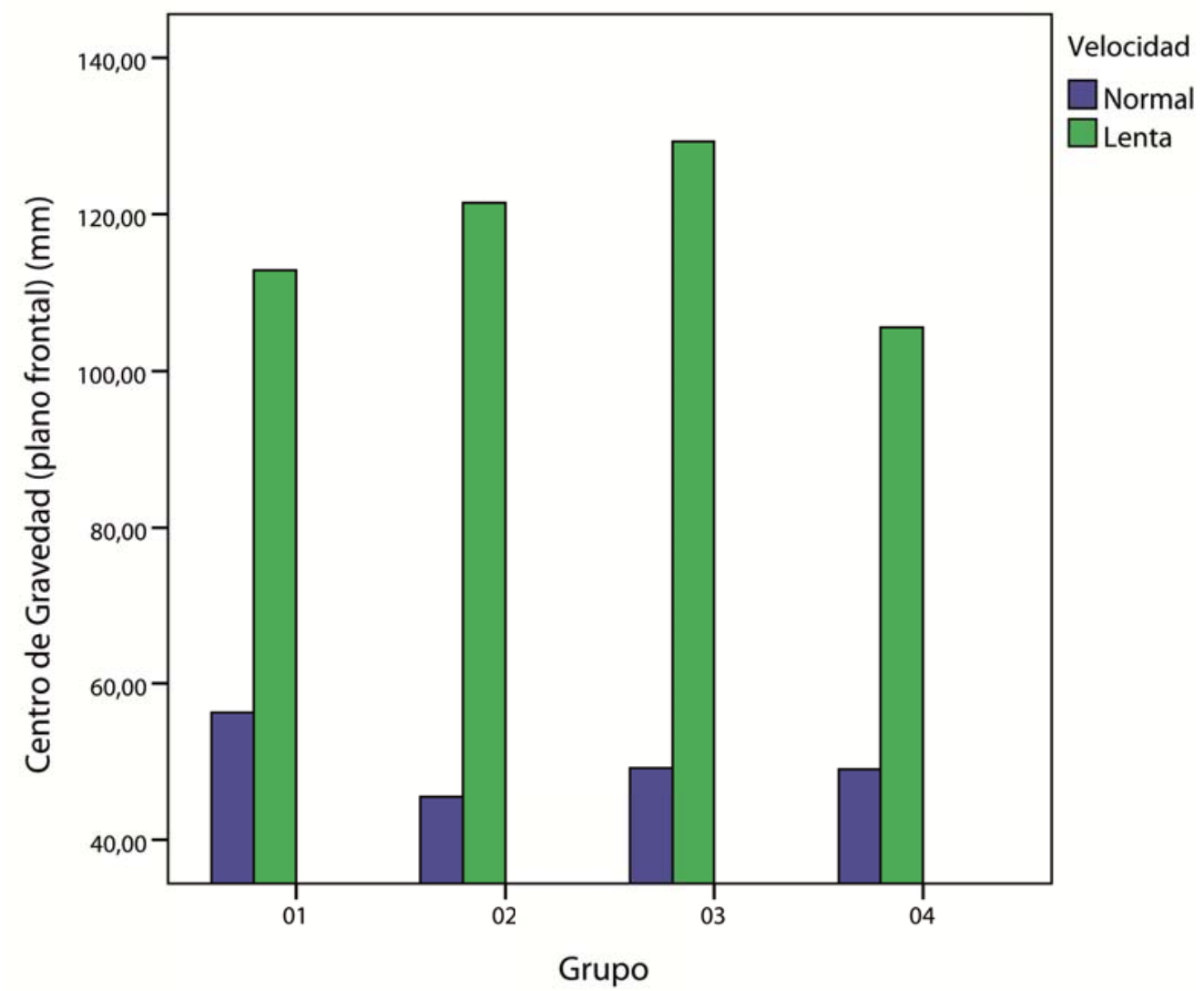

Figura 45. Centro de gravedad (plano frontal) en mujeres (Velocidad Normal y Velocidad Lenta) 
Tabla 22. Parámetros Cinemáticos: Pelvis y Centro de Gravedad (Velocidad Normal y Velocidad Lenta)

\begin{tabular}{|c|c|c|c|c|c|c|c|c|c|c|c|}
\hline & \multicolumn{2}{|c|}{$\mathrm{H} 1$} & \multicolumn{2}{|c|}{$\mathrm{H} 2$} & \multicolumn{2}{|c|}{ H3 } & \multicolumn{2}{|c|}{$\mathrm{H} 4$} & \multirow[b]{2}{*}{ Velocidad } & \multirow[b]{2}{*}{ Grupo } & \multirow[b]{2}{*}{ Inter. } \\
\hline & NORMAL & LENTA & NORMAL & LENTA & NORMAL & LENTA & NORMAL & LENTA & & & \\
\hline & Media \pm DS & Media \pm DS & Media \pm DS & Media \pm DS & Media \pm DS & Media \pm DS & Media \pm DS & Media \pm DS & $F_{1,29}$ & $\mathrm{~F}_{3,29}$ & $F_{3,29}$ \\
\hline CG plano sagital $(\mathrm{mm})^{*+}$ & $41,28 \pm 5,17$ & $15,83 \pm 2,83$ & $48,82 \pm 9,08$ & $20,01 \pm 4,86$ & $37,95 \pm 5,45$ & $16,82 \pm 4,20$ & $47,25 \pm 9,41$ & $18,66 \pm 5,76$ & 228,91 & 5,33 & 1,11 \\
\hline Pelvis plano frontal $\left({ }^{\circ}\right)^{*}$ & $12,05 \pm 3,41$ & $8,27 \pm 2,35$ & $12,43 \pm 3,43$ & $8,82 \pm 0,73$ & $9,63 \pm 2,94$ & $7,90 \pm 1,46$ & $12,69 \pm 3,47$ & $8,39 \pm 1,42$ & 49,31 & 0,99 & 1,31 \\
\hline Pelvis plano sagital $\left({ }^{\circ}\right)^{*}$ & $2,79 \pm 0,68$ & $3,66 \pm 0,84$ & $3,74 \pm 1,23$ & $4,88 \pm 1,30$ & $3,43 \pm 0,84$ & $3,54 \pm 0,74$ & $3,44 \pm 0,96$ & $3,85 \pm 1,60$ & 6,03 & 1,99 & 0,67 \\
\hline \multirow[t]{4}{*}{ Pelvis plano transversal $\left({ }^{\circ}\right)$} & $9,51 \pm 2,29$ & $9,69 \pm 1,83$ & $9,41 \pm 3,97$ & $9,56 \pm 3,36$ & $8,58 \pm 1,92$ & $11,00 \pm 2,20$ & $9,44 \pm 3,02$ & $11,52 \pm 3,73$ & 3,15 & 0,40 & 0,74 \\
\hline & \multicolumn{2}{|c|}{ M1 } & \multicolumn{2}{|c|}{ M2 } & \multicolumn{2}{|c|}{ M3 } & \multicolumn{2}{|c|}{ M4 } & & & \\
\hline & NORMAL & LENTA & NORMAL & LENTA & NORMAL & LENTA & NORMAL & LENTA & Velocidad & Grupo & Inter. \\
\hline & Media \pm DS & Media \pm DS & Media \pm DS & Media \pm DS & Media \pm DS & Media \pm DS & Media \pm DS & Media \pm DS & $F_{1,25}$ & $\mathrm{~F}_{3,25}$ & $F_{3,25}$ \\
\hline CG plano frontal $(\mathrm{mm})^{*} \ddagger$ & $56,36 \pm 7,92$ & $112,81 \pm 12,82$ & $45,48 \pm 11092$ & $121,45 \pm 19,80$ & $49,25 \pm 8,79$ & $129,38 \pm 12,18$ & $49,11 \pm 11,14$ & $105,59 \pm 16,19$ & 704,58 & 1,50 & 7,48 \\
\hline CG plano sagital $(\mathrm{mm})^{*}$ & $42,86 \pm 13,10$ & $15,22 \pm 2,25$ & $44,33 \pm 10,50$ & $16,32 \pm 3,49$ & $46,07 \pm 11,41$ & $19,18 \pm 4,77$ & $44,44 \pm 9,56$ & $16,21 \pm 3,25$ & 193,03 & 0,34 & 0,03 \\
\hline Pelvis plano frontal $\left({ }^{\circ}\right)^{*}$ & $13,50 \pm 0,36$ & $8,53 \pm 1,53$ & $15,15 \pm 2,56$ & $8,89 \pm 2,03$ & $16,19 \pm 4,59$ & $9,25 \pm 1,77$ & $15,68 \pm 3,15$ & $10,88 \pm 2,38$ & 76,16 & 0,88 & 0,79 \\
\hline Pelvis plano sagital $\left({ }^{\circ}\right)^{*}$ & $2,24 \pm 0,42$ & $2,93 \pm 0,40$ & $3,85 \pm 0,78$ & $4,04 \pm 1,27$ & $3,57 \pm 1,35$ & $4,51 \pm 1,40$ & $3,21 \pm 0,83$ & $4,55 \pm 1,02$ & 6,80 & 2,61 & 0,89 \\
\hline Pelvis plano transversal $\left({ }^{\circ}\right)$ & $6,48 \pm 1,59$ & $8,82 \pm 2,05$ & $11,11 \pm 3,25$ & $9,50 \pm 2,73$ & $9,29 \pm 2,90$ & $12,17 \pm 3,91$ & $9,49 \pm 2,78$ & $11,10 \pm 3,24$ & 1,96 & 1,68 & 1,53 \\
\hline
\end{tabular}


El movimiento del centro de gravedad en el plano sagital en las mujeres disminuyó un $60 \%$ en la velocidad lenta $(p<0,001)$, no presentó diferencias entre grupos de estatura $(p=0,795)$ y no hubo efecto de la interacción entre velocidad y grupo en esta variable $(p=0,992)$. El movimiento de la pelvis en el plano frontal disminuyó un $36 \%$ en la velocidad lenta $(p<0,001)$, mientras que el movimiento de la pelvis en el plano sagital aumento un $34 \%(p=0,015)$. En ninguna de las dos variables se presentaron diferencias entre grupos $(p=0,464$ para el movimiento de la pelvis en el plano frontal; $p=0,074$ para el movimiento de la pelvis en el plano sagital), ni hubo efecto de la interacción entre velocidad y grupo ( $p=0,510$ para el movimiento de la pelvis en el plano frontal; $p=0,458$ para el movimiento de la pelvis en el plano sagital). El movimiento de la pelvis en el plano transversal en las mujeres no mostró diferencias entre velocidad normal y lenta $(p=0,174)$, ni entre grupos de estatura $(p=0,196)$. Tampoco se presentó efecto de la interacción entre velocidad y grupo en el movimiento de la pelvis en el plano transversal en las mujeres $(p=0,231)$ (Tabla 22).

\subsubsection{Cadera}

Los hombres mostraron una disminución del 11\% (aproximadamente $5^{\circ}$ ) en la flexión máxima de cadera $\left({ }^{\circ}\right)$ en la velocidad lenta comparándola con la velocidad normal $(p<0,001)$, además de un retraso del $2,5 \%$ en el instante de flexión máxima de cadera (\%) en el ciclo de la marcha $(p=0,001)$. No se mostraron diferencias entre grupos en la flexión máxima de cadera $(p=0,184)$ ni en el porcentaje del ciclo en el que se encuentra la flexión máxima de cadera $(p=0,462)$. Tampoco se presentó efecto de la interacción entre velocidad y grupo de estatura en ninguna de las dos variables ( $p=0,858$ para la flexión máxima de cadera; $p=0,360$ para el porcentaje del ciclo en el que se encuentra la flexión máxima de cadera). La extensión máxima de cadera $\left({ }^{\circ}\right)$ en los hombres sufrió una disminución de aproximadamente $8^{\circ}$ en la velocidad lenta $(p<0,001)$. También se produjo un retraso del $8,6 \%$ en el instante de extensión máxima de cadera (\%) en el ciclo de la marcha $(p<0,001)$. No se mostraron diferencias en las comparaciones por grupos de estatura en la extensión máxima de cadera $(p=0,599)$ ni en el porcentaje del ciclo en el que sucede la extensión máxima de cadera $(p=0,498)$. No hubo efecto de la interacción entre velocidad y grupo de estatura en ninguna de estas dos variables ( $p=0,579$ para la extensión máxima de cadera; $p=0,059$ para el porcentaje del ciclo en el que se encuentra la extensión máxima de cadera). En el contacto inicial del ciclo de la marcha los hombres mostraron una disminución de $6^{\circ}$ en la flexión de cadera (aproximadamente el 15\%) en la velocidad lenta $(p<0,001)$. La flexión de cadera durante el contacto inicial no mostró diferencias entre grupos de estatura $(p=0,108)$, ni presentó efecto de la interacción entre velocidad y grupo $(p=0,997)$ (Tabla 23$)$. 
Las mujeres presentaron en los parámetros de la cadera diferencias similares a las encontradas en los hombres. La flexión máxima de cadera $\left({ }^{\circ}\right)$ en las mujeres disminuyó un $11 \%$ (aproximadamente $5,5^{\circ}$ ) en la velocidad lenta en comparación con la normal $(p<0,001)$, y además se mostró un retraso del $5 \%$ en el instante del ciclo (\%) en el que sucede la flexión máxima de cadera $(p<0,001)$. No se mostraron diferencias entre los grupos de estatura en la flexión máxima de cadera $(p=0,897)$ ni en el porcentaje del ciclo en el que se encuentra dicha flexión máxima $(p=0,965)$. Tampoco hubo efecto de la interacción entre velocidad y grupo de estatura para estas variables $(p=0,363$ para la flexión máxima de cadera; $p=0,859$ para el porcentaje del ciclo en el que se encuentra la flexión máxima) (Tabla 23).

La extensión máxima de cadera $\left(^{\circ}\right)$ en las mujeres disminuyó $7^{\circ}(p<0,001)$, alcanzando menor extensión durante la velocidad lenta. Además, al igual que en los hombres, el instante del ciclo en el que sucede la extensión máxima (\%) sufrió un retraso del 10,5\% ( $p<0,001)$. La extensión máxima de cadera $(p=0,901)$ y el porcentaje del ciclo en el que se encuentra la extensión máxima $(p=0,646)$ no mostraron diferencias entre grupos de estatura. Tampoco hubo efecto de la interacción entre velocidad y grupo para estas variables $(p=0,157$ para la extensión máxima de cadera; $p=0,571$ para el porcentaje del ciclo en el que se sucede la extensión máxima de cadera). La flexión de cadera en el contacto inicial en las mujeres disminuyó $7^{\circ}$ (aproximadamente un 14\%) durante la velocidad lenta $(p<0,001)$, no presentando diferencias entre grupos de estatura $(p=0,973)$ ni efecto de la interacción entre velocidad y grupo $(p=0,627)$ (Tabla 23). 
Tabla 23. Parámetros Cinemáticos: Cadera (Velocidad Normal y Velocidad Lenta)

\begin{tabular}{|c|c|c|c|c|c|c|c|c|c|c|c|}
\hline & \multicolumn{2}{|c|}{$\mathrm{H} 1$} & \multicolumn{2}{|c|}{$\mathrm{H} 2$} & \multicolumn{2}{|c|}{ H3 } & \multicolumn{2}{|c|}{$\mathrm{H} 4$} & \multirow[b]{2}{*}{ Velocidad } & \multirow[b]{2}{*}{ Grupo } & \multirow[b]{2}{*}{ Inter. } \\
\hline & NORMAL & LENTA & NORMAL & LENTA & NORMAL & LENTA & NORMAL & LENTA & & & \\
\hline & Media \pm DS & Media \pm DS & Media \pm DS & Media \pm DS & Media \pm DS & Media \pm DS & Media \pm DS & Media \pm DS & $F_{1,29}$ & $F_{3,29}$ & $F_{3,29}$ \\
\hline Flexión Máxima Cadera $\left({ }^{\circ}\right)^{*}$ & $43,06 \pm 6,53$ & $39,37 \pm 8,81$ & $46,02 \pm 6,80$ & $41,09 \pm 6,31$ & $39,44 \pm 4,58$ & $34,44 \pm 3,94$ & $43,55 \pm 4,56$ & $38,35 \pm 4,02$ & 38,778 & 1,723 & 0,253 \\
\hline Flexión Máxima Cadera (\%)* & $87,14 \pm 2,67$ & $90,57 \pm 4,06$ & $88,53 \pm 4,02$ & $89,22 \pm 2,18$ & $89,91 \pm 5,16$ & $91,03 \pm 2,79$ & $87,04 \pm 2,14$ & $90,96 \pm 2,51$ & 7,576 & 0,881 & 1,113 \\
\hline Extensión Máxima Cadera $\left({ }^{\circ}\right)^{*}$ & $-3,01 \pm 4,30$ & $5,75 \pm 4,30$ & $0,01 \pm 9,56$ & $6,94 \pm 9,68$ & $-3,37 \pm 4,31$ & $3,52 \pm 6,52$ & $-1,21 \pm 5,08$ & $8,07 \pm 5,16$ & 79,577 & 0,634 & 0,668 \\
\hline Extensión Máxima Cadera (\%)* & $50,81 \pm 1,33$ & $59,32 \pm 3,64$ & $51,62 \pm 0,92$ & $60,96 \pm 3,77$ & $51,50 \pm 1,29$ & $56,82 \pm 3,98$ & $49,84 \pm 1,46$ & $60,41 \pm 4,73$ & 162,009 & 0,811 & 2,783 \\
\hline \multirow[t]{4}{*}{ Flexión Cadera Contacto Inicial $\left({ }^{\circ}\right)^{*}$} & $39,53 \pm 4,36$ & $33,40 \pm 4,35$ & $42,70 \pm 7,16$ & $37,30 \pm 7,69$ & $36,17 \pm 5,32$ & $30,27 \pm 3,90$ & $39,75 \pm 4,48$ & $33,65 \pm 3,73$ & 57,005 & 2,209 & 0,016 \\
\hline & \multicolumn{2}{|c|}{ M1 } & \multicolumn{2}{|c|}{ M2 } & \multicolumn{2}{|c|}{ M3 } & \multicolumn{2}{|c|}{ M4 } & & & \\
\hline & NORMAL & LENTA & NORMAL & LENTA & NORMAL & LENTA & NORMAL & LENTA & Velocidad & Grupo & Inter. \\
\hline & Media \pm DS & Media \pm DS & Media \pm DS & Media \pm DS & Media \pm DS & Media \pm DS & Media \pm DS & Media \pm DS & $\mathrm{F}_{1,25}$ & $F_{3,25}$ & $F_{3,25}$ \\
\hline Flexión Máxima Cadera $\left({ }^{\circ}\right)^{*}$ & $50,07 \pm 3,69$ & $43,00 \pm 3,58$ & $51,76 \pm 7,38$ & $44,63 \pm 9,47$ & $52,38 \pm 7,53$ & $47,36 \pm 7,34$ & $50,88 \pm 5,98$ & $46,38 \pm 6,32$ & 70,811 & 0,197 & 1,112 \\
\hline Flexión Máxima Cadera (\%)* & $87,04 \pm 2,40$ & $90,57 \pm 1,18$ & $86,62 \pm 3,76$ & $90,89 \pm 1,59$ & $85,42 \pm 6,66$ & $90,94 \pm 2,70$ & $85,68 \pm 1,42$ & $91,09 \pm 1,26$ & 26,789 & 0,091 & 0,252 \\
\hline Extensión Máxima Cadera $\left({ }^{\circ}\right)^{*}$ & $5,63 \pm 2,62$ & $12,56 \pm 2,99$ & $1,36 \pm 10,51$ & $10,61 \pm 7,66$ & $4,06 \pm 6,39$ & $10,70 \pm 6,04$ & $3,73 \pm 4,59$ & $9,53 \pm 4,83$ & 125,878 & 0,192 & 1,889 \\
\hline Extensión Máxima Cadera (\%)* & $52,42 \pm 0,78$ & $60,62 \pm 3,19$ & $50,15 \pm 0,85$ & $61,37 \pm 3,69$ & $50,35 \pm 1,08$ & $60,88 \pm 2,55$ & $50,98 \pm 1,33$ & $61,69 \pm 2,07$ & 243,271 & 0,561 & 0,682 \\
\hline Flexión Cadera Contacto Inicial $\left({ }^{\circ}\right)^{*}$ & $48,11 \pm 3,97$ & $39,29 \pm 3,08$ & $47,69 \pm 7,24$ & $40,29 \pm 9,28$ & $48,39 \pm 7,47$ & $42,08 \pm 6,51$ & $46,90 \pm 7,47$ & $41,02 \pm 6,27$ & 85,376 & 0,075 & 0,591 \\
\hline
\end{tabular}
Hombres: H1 (<25\%), H2 (25\%-50\%), H3 (50\%-75\%), H4 (>75\%); Mujeres: M1 (<25\%), M2 (25\%-50\%), M3 (50\%-75\%), M4 (>75\%) 


\subsubsection{Rodilla}

Los hombres presentaron una disminución del 15\% (aproximadamente $10^{\circ}$ ) en la flexión máxima de rodilla $\left({ }^{\circ}\right)$ durante la velocidad lenta en comparación con la velocidad normal $(p<0,001)$. No se encontraron diferencias entre los grupos de estatura $(p=0,896)$, ni hubo efecto de la interacción entre velocidad y grupo en la flexión máxima de rodilla $(p=0,546)$. El porcentaje del ciclo en el que sucede la flexión máxima de rodilla se retrasó un 9\% de media en los hombres durante la velocidad lenta $(p<0,001)$. No se presentaron diferencias entre los grupos de estatura $(p=0,541)$, pero sí hubo efecto de la interacción de la velocidad y grupo $(p=0,019)$, mostrando un aumento mayor en los grupos de los extremos ( $\mathrm{H} 1$ y $\mathrm{H} 4)$ que en los centrales ( $\mathrm{H} 2$ y H3) (Figura 46) (Tabla 24).

Los valores de extensión máxima de rodilla $\left(^{\circ}\right)$ en los hombres aumentaron un $50 \%$ en la velocidad lenta, lo que se tradujo en un aumento de $2^{\circ}, \mathrm{y}$, por lo tanto, en una menor extensión de rodilla $(p=0,001)$. Además, el instante del ciclo en el que sucede la extensión máxima de rodilla también se retrasó un $6 \%$ durante la velocidad lenta $(p<0,001)$. No se mostraron diferencias entre los grupos de estatura para la extensión máxima de rodilla $(p=0,916)$ ni para el porcentaje del ciclo en el que se encuentra dicha extensión máxima $(p=0,071)$. Tampoco se presentó, para estas variables, efecto de la interacción entre velocidad y grupo de estatura ( $p=0,806$ para la extensión máxima de rodilla; $p=0,580$ para el porcentaje del ciclo en el que sucede la extensión máxima de rodilla). La flexión de rodilla en el contacto inicial aumentó un $1^{\circ}$ de media (13\%) durante la velocidad lenta en los hombres $(p=0,012)$. No se mostraron diferencias en la flexión de rodilla en el contacto inicial durante las comparaciones por grupos $(p=0,463)$, ni hubo efecto de la interacción de velocidad y grupo en la misma variable $(p=0,055)$ (Tabla 24$)$. 
Tabla 24. Parámetros Cinemáticos: Rodilla (Velocidad Normal y Velocidad Lenta)

\begin{tabular}{|c|c|c|c|c|c|c|c|c|c|c|c|}
\hline & \multicolumn{2}{|c|}{$\mathrm{H} 1$} & \multicolumn{2}{|c|}{$\mathrm{H} 2$} & \multicolumn{2}{|c|}{ H3 } & \multicolumn{2}{|c|}{$\mathrm{H} 4$} & \multirow[b]{2}{*}{ Velocidad } & \multirow[b]{2}{*}{ Grupo } & \multirow[b]{2}{*}{ Inter. } \\
\hline & NORMAL & LENTA & NORMAL & LENTA & NORMAL & LENTA & NORMAL & LENTA & & & \\
\hline & Media \pm DS & Media \pm DS & Media \pm DS & Media \pm DS & Media \pm DS & Media \pm DS & Media \pm DS & Media \pm DS & $F_{1,29}$ & $F_{3,29}$ & $F_{3,29}$ \\
\hline Flexión Máxima Rodilla (º) & $65,95 \pm 5,83$ & $58,54 \pm 12,05$ & $66,00 \pm 4,59$ & $54,56 \pm 8,60$ & $66,14 \pm 3,11$ & $55,59 \pm 5,88$ & $66,94 \pm 3,27$ & $54,48 \pm 3,39$ & 59,83 & 0,20 & 0,72 \\
\hline Flexión Máxima Rodilla (\%)*‡ & $70,80 \pm 1,54$ & $80,07 \pm 2,57$ & $70,81 \pm 1,08$ & $78,56 \pm 1,44$ & $70,93 \pm 1,27$ & $78,56 \pm 3,48$ & $69,87 \pm 1,01$ & $81,24 \pm 2,99$ & 354,51 & 0,73 & 3,89 \\
\hline Extensión Máxima Rodilla $\left({ }^{\circ}\right)^{*}$ & $4,96 \pm 3,80$ & $7,65 \pm 4,64$ & $5,56 \pm 5,34$ & $7,80 \pm 3,31$ & $5,90 \pm 4,34$ & $7,11 \pm 4,12$ & $6,57 \pm 3,36$ & $8,36 \pm 3,96$ & 12,50 & 0,17 & 0,33 \\
\hline Extensión Máxima Rodilla (\%)* & $39,71 \pm 1,44$ & $46,01 \pm 2,07$ & $38,58 \pm 1,31$ & $45,25 \pm 3,75$ & $38,19 \pm 2,65$ & $43,12 \pm 2,43$ & $38,08 \pm 1,16$ & $44,04 \pm 1,90$ & 190,18 & 2,61 & 0,67 \\
\hline \multirow[t]{4}{*}{ Flexión Rodilla Contacto Inicial $\left({ }^{\circ}\right)^{*}$} & $8,11 \pm 3,27$ & $10,85 \pm 4,02$ & $7,24 \pm 4,60$ & $8,28 \pm 3,63$ & $10,10 \pm 3,63$ & $11,42 \pm 3,96$ & $9,27 \pm 3,74$ & $8,79 \pm 3,37$ & 7,15 & 0,88 & 2,85 \\
\hline & \multicolumn{2}{|c|}{ M1 } & \multicolumn{2}{|c|}{ M2 } & \multicolumn{2}{|c|}{ M3 } & \multicolumn{2}{|c|}{ M4 } & & & \\
\hline & NORMAL & LENTA & NORMAL & LENTA & NORMAL & LENTA & NORMAL & LENTA & Velocidad & Grupo & Inter. \\
\hline & Media \pm DS & Media \pm DS & Media \pm DS & Media \pm DS & Media \pm DS & Media \pm DS & Media \pm DS & Media \pm DS & $\mathrm{F}_{1,25}$ & $F_{3,25}$ & $F_{3,25}$ \\
\hline Flexión Máxima Rodilla $\left({ }^{\circ}\right)^{*+}$ & $62,82 \pm 2,21$ & $53,60 \pm 0,36$ & $69,22 \pm 3,23$ & $59,88 \pm 4,74$ & $69,41 \pm 4,63$ & $61,72 \pm 5,35$ & $69,60 \pm 4,48$ & $62,91 \pm 4,80$ & 120,95 & 3,15 & 0,88 \\
\hline Flexión Máxima Rodilla $(\%)^{* \ddagger}$ & $72,32 \pm 0,44$ & $78,71 \pm 4,71$ & $70,69 \pm 1,43$ & $81,63 \pm 1,71$ & $70,81 \pm 1,71$ & $83,05 \pm 3,11$ & $71,63 \pm 0,95$ & $81,91 \pm 1,22$ & 345,55 & 0,89 & 3,90 \\
\hline Extensión Máxima Rodilla $\left({ }^{\circ}\right) \ddagger$ & $8,17 \pm 1,82$ & $9,14 \pm 1,90$ & $7,14 \pm 4,13$ & $12,42 \pm 5,04$ & $8,57 \pm 2,75$ & $9,55 \pm 2,96$ & $8,34 \pm 2,48$ & $6,79 \pm 2,68$ & 3,73 & 0,95 & 5,27 \\
\hline Extensión Máxima Rodilla (\%)* & $38,12 \pm 2,60$ & $41,24 \pm 4,66$ & $37,70 \pm 1,90$ & $42,80 \pm 4,09$ & $37,36 \pm 2,18$ & $45,23 \pm 4,02$ & $38,98 \pm 2,15$ & $41,88 \pm 6,08$ & 19,13 & 0,38 & 1,50 \\
\hline Flexión Rodilla Contacto Inicial ( $\left.{ }^{\circ}\right)^{*}$ & $10,82 \pm 1,24$ & $8,85 \pm 2,56$ & $11,17 \pm 2,48$ & $11,35 \pm 4,94$ & $13,37 \pm 4,61$ & $10,41 \pm 3,13$ & $12,20 \pm 2,87$ & $10,94 \pm 2,95$ & 4,50 & 0,35 & 1,26 \\
\hline
\end{tabular}




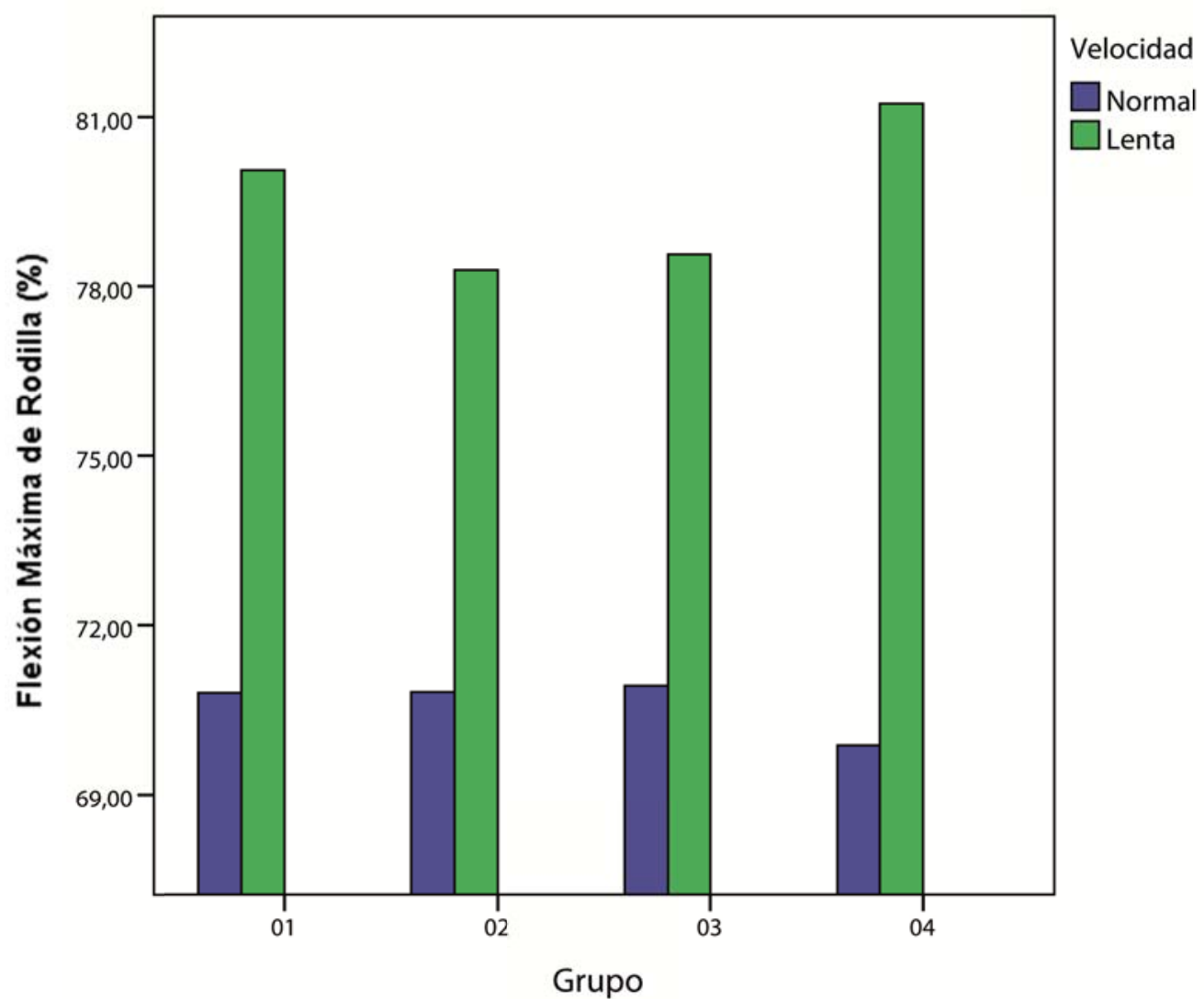

Figura 46. Flexión Máxima de Rodilla (\%) en hombres (Velocidad Normal y Velocidad Lenta)

Las mujeres mostraron una disminución del 11,5\% (aproximadamente $8^{\circ}$ ) en la flexión máxima de rodilla en la velocidad lenta $(p<0,001)$. Además, se presentaron diferencias entre grupos de estatura $(p=0,043)$, siendo los valores del grupo por debajo del percentil 25 (M1) menores que los del grupo por encima del percentil 75 (M4) $(p=0,038)$. No existió efecto de la interacción entre velocidad y grupo para la flexión máxima de rodilla $(p=0,464)$. El porcentaje del ciclo en el que sucede la flexión máxima de rodilla (\%) se retrasó un 10,5\% de media en las mujeres durante la velocidad lenta $(p<0,001)$. No se presentaron diferencias entre los grupos de estatura $(p=0,459)$, pero hubo efecto de la interacción de la velocidad y grupo $(p=0,020)$, mostrando un aumento mayor en los grupos centrales (M2 y M3) que en los extremos (M1 y M4), al contrario de los que encontrábamos en los hombres (Figura 47). La extensión máxima de rodilla en las mujeres no presentó diferencias entre la velocidad lenta y la velocidad normal $(p=0,065)$ y tampoco en las comparaciones entre grupos de estatura $(p=0,433)$. Se mostró efecto de la interacción entre velocidad y grupo $(p=0,006)$, siendo $M 2$ el grupo que presentaba un mayor aumento en los valores de extensión máxima de rodilla, y alcanzando menor extensión que el resto de los grupos, durante la velocidad lenta $(p<0,001)$. El instante de extensión máxima de rodilla en el ciclo de la marcha se retrasó un $5 \%$ durante la velocidad 
lenta $(p<0,001)$, no mostró diferencias entre los grupos de estatura $(p=0,766)$ y no presentó efecto de la interacción entre velocidad y grupo $(p=0,240)$. La flexión de rodilla en las mujeres durante el contacto inicial disminuyó levemente un 10\% (aproximadamente $1^{\circ}$ ) en la velocidad normal $(p=0,044)$. No se mostraron diferencias entre grupos $(p=0,788)$, ni hubo efecto de la interacción entre velocidad y grupo en la flexión de rodilla durante el contacto inicial $(p=0,310)$ (Tabla 24).

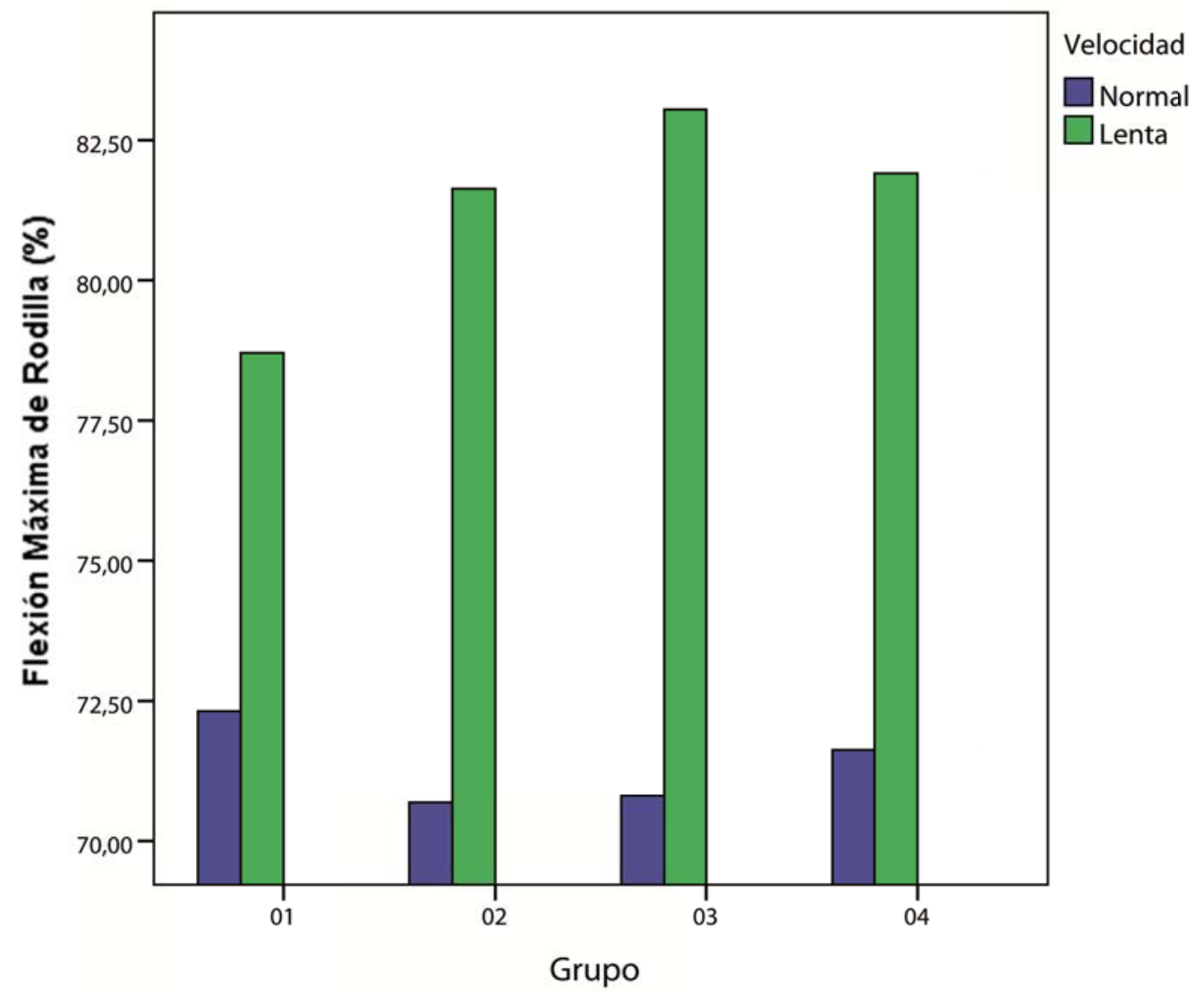

Figura 47. Flexión Máxima de Rodilla (\%) en mujeres (Velocidad Normal y Velocidad Lenta)

\subsubsection{Tobillo}

Los hombres presentaron un aumento del $6 \%$ en la flexión máxima de tobillo $\left(^{\circ}\right)$ durante la velocidad lenta $(p=0,038)$ y un retraso del $8,5 \%$ en el porcentaje del ciclo en el que se encuentra la flexión máxima de tobillo $(p<0,001)$. Ninguna de las dos variables presentaron diferencias en las comparaciones entre grupos $(p=0,490$ para la flexión máxima de tobillo; $p=0,365$ para el porcentaje del ciclo en el que sucede la flexión máxima de tobillo). No se mostró efecto de la interacción entre velocidad y grupo de estatura en la flexión máxima de tobillo $(p=0,724)$, ni en el porcentaje del ciclo en el que se encuentra la flexión máxima de tobillo en los hombres ( $p=0,323)$. Los valores de extensión máxima de tobillo aumentaron de 
media $6^{\circ}$, alcanzando, por tanto, menor extensión de tobillo durante la velocidad lenta $(p<0,001)$. No se presentaron diferencias entre grupos de estatura $(p=0,185)$ y tampoco hubo efecto de la interacción entre la velocidad y el grupo en la extensión máxima de tobillo $(p=0,927)$. El instante del ciclo en el que sucede la extensión máxima de tobillo se presentó atrasado un $13 \%$ en la velocidad lenta en comparación con la velocidad normal $(p<0,001)$. No se mostraron diferencias entre grupos de estatura $(p=0,677)$, y se presentó un efecto de la interacción entre velocidad y grupo en el porcentaje del ciclo en el que se encuentra la extensión máxima de tobillo en los hombres $(p=0,022)$. Siendo el retraso del instante de extensión máxima de tobillo en el ciclo mayor en los grupos extremos ( $\mathrm{H} 1$ y $\mathrm{H} 4)$ que en los grupos centrales ( $\mathrm{H} 2$ y H3) (Figura 48). La flexión de tobillo durante el contacto inicial no presentó diferencias entre la velocidad normal y lenta $(p=0,872)$, ni por grupo de estatura $(p=0,705)$, ni tampoco en la interacción entre velocidad y grupo en la flexión de tobillo durante el contacto inicial $(p=0,781)$ (Tabla 25$)$.

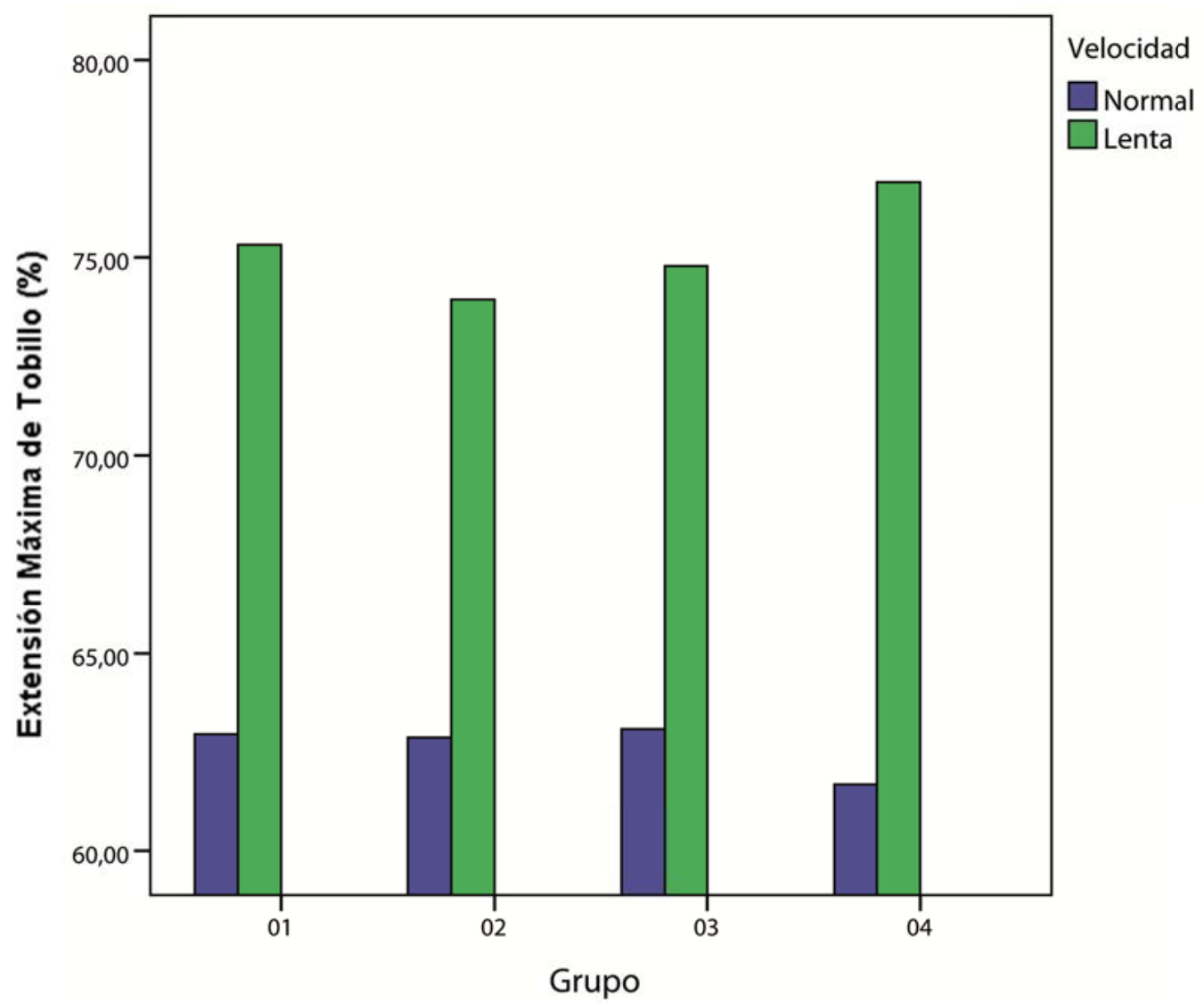

Figura 48. Extensión Máxima de Tobillo (\%) en hombres (Velocidad Normal y Velocidad Lenta)

Las mujeres no mostraron diferencias en la flexión máxima de tobillo $\left({ }^{\circ}\right)$ entre velocidad normal y velocidad lenta $(p=0,596)$, ni entre grupos de estatura $(p=0,484)$. Sin embargo, se 
presentó un efecto de la interacción entre velocidad y grupo en la flexión máxima de tobillo $(p=0,042)$, mostrándose mayor flexión máxima de tobillo en el grupo M2 durante la velocidad lenta $(p=0,009)$ (Tabla 25).

El instante del ciclo en el que sucede la flexión máxima de tobillo se encontró retrasado un $6 \%$ de media en la velocidad lenta en comparación con la velocidad normal $(p=0,019)$. No se presentaron diferencias entre los grupos de estatura $(p=0,929)$, ni efecto de la interacción entre velocidad y grupo para el porcentaje del ciclo en el que se encuentra la flexión máxima de tobillo en las mujeres $(p=0,668)$. La extensión máxima de tobillo $\left({ }^{\circ}\right)$ no presentó diferencias entre velocidad normal y velocidad lenta $(p=0,281)$, ni en las comparaciones por grupos $(p=0,285)$. Tampoco se mostró efecto de la interacción entre velocidad y grupo en la extensión máxima de tobillo en las mujeres $(p=0,236)$. El instante del ciclo (\%) en el que se encuentra la extensión máxima de tobillo se retrasó un $15 \%$ en las mujeres durante la velocidad lenta $(p<0,001)$, aunque no mostró diferencias entre los grupos de estatura $(p=0,071)$, ni en la interacción entre la velocidad y el grupo en el porcentaje del ciclo en el que sucede la extensión máxima de tobillo $(\mathrm{p}=0,057)$. La flexión de tobillo no mostró diferencias durante el contacto inicial en las comparaciones entre velocidad normal y lenta $(p=0,689)$, ni en las comparaciones por grupo $(p=0,226)$. Tampoco se mostró efecto de la interacción entre velocidad y grupo en la flexión de tobillo durante el contacto inicial $(p=0,122)$ (Tabla 25). 
Tabla 25. Parámetros Cinemáticos: Tobillo (Velocidad Normal y Velocidad Lenta)

\begin{tabular}{|c|c|c|c|c|c|c|c|c|c|c|c|}
\hline & \multicolumn{2}{|c|}{$\mathrm{H} 1$} & \multicolumn{2}{|c|}{$\mathrm{H} 2$} & \multicolumn{2}{|c|}{ H3 } & \multicolumn{2}{|c|}{$\mathrm{H} 4$} & \multirow[b]{2}{*}{ Velocidad } & \multirow[b]{2}{*}{ Grupo } & \multirow[b]{2}{*}{ Inter } \\
\hline & NORMAL & LENTA & NORMAL & LENTA & NORMAL & LENTA & NORMAL & LENTA & & & \\
\hline & Media \pm DS & Media \pm DS & Media \pm DS & Media \pm DS & Media \pm DS & Media \pm DS & Media \pm DS & Media \pm DS & $\mathrm{F}_{1,29}$ & $F_{3,29}$ & $\mathrm{~F}_{3,29}$ \\
\hline Flexión Máxima Tobillo $\left({ }^{\circ}\right)^{*}$ & $15,82 \pm 2,05$ & $16,86 \pm 1,63$ & $15,92 \pm 3,12$ & $15,80 \pm 1,89$ & $16,40 \pm 3,71$ & $17,99 \pm 3,11$ & $17,28 \pm 2,21$ & $17,93 \pm 2,76$ & 4,71 & 0,82 & 0,44 \\
\hline Flexión Máxima Tobillo (\%)* & $44,10 \pm 2,97$ & $52,06 \pm 2,71$ & $44,17 \pm 1,63$ & $51,39 \pm 2,55$ & $47,45 \pm 6,1$ & $53,38 \pm 2,71$ & $42,47 \pm 5,49$ & $53,16 \pm 4,22$ & 83,42 & 1,10 & 1,21 \\
\hline Extensión Máxima Tobillo $\left({ }^{\circ}\right)^{*}$ & $-15,15 \pm 3,52$ & $-8,11 \pm 8,33$ & $-14,90 \pm 5,01$ & $-9,03 \pm 5,73$ & $-9,94 \pm 3,26$ & $-3,24 \pm 9,14$ & $-10,70 \pm 4,37$ & $-5,80 \pm 9,19$ & 19,81 & 1,72 & 0,15 \\
\hline Extensión Máxima Tobillo (\%)*‡ & $62,96 \pm 1,40$ & $75,32 \pm 3,05$ & $62,87 \pm 1,46$ & $74,16 \pm 1,88$ & $63,09 \pm 1,21$ & $74,79 \pm 2,54$ & $61,69 \pm 1,36$ & $76,91 \pm 2,66$ & 637,16 & 0,51 & 3,74 \\
\hline \multirow[t]{4}{*}{ Flexión Tobillo Contacto Inicial ( $\left(^{\circ}\right)$} & $3,98 \pm 2,23$ & $4,75 \pm 4,30$ & $3,81 \pm 2,56$ & $5,08 \pm 2,13$ & $5,71 \pm 2,92$ & $4,87 \pm 7,35$ & $6,06 \pm 3,06$ & $5,73 \pm 4,31$ & 0,03 & 0,47 & 0,36 \\
\hline & \multicolumn{2}{|c|}{ M1 } & \multicolumn{2}{|c|}{ M2 } & \multicolumn{2}{|c|}{ M3 } & \multicolumn{2}{|c|}{ M4 } & & & \\
\hline & NORMAL & LENTA & NORMAL & LENTA & NORMAL & LENTA & NORMAL & LENTA & Velocidad & Grupo & Inter. \\
\hline & Media \pm DS & Media \pm DS & Media \pm DS & Media \pm DS & Media \pm DS & Media \pm DS & Media \pm DS & Media \pm DS & $F_{1,25}$ & $\mathrm{~F}_{3,25}$ & $\mathrm{~F}_{3,25}$ \\
\hline Flexión Máxima Tobillo $\left({ }^{\circ}\right) \ddagger$ & $17,37 \pm 3,60$ & $17,17 \pm 2,67$ & $15,22 \pm 4,42$ & $17,70 \pm 2,77$ & $16,82 \pm 3,36$ & $16,74 \pm 3,69$ & $15,47 \pm 2,02$ & $14,36 \pm 2,38$ & 0,29 & 0,84 & 3,17 \\
\hline Flexión Máxima Tobillo (\%)* & $43,74 \pm 2,55$ & $51,78 \pm 3,45$ & $47,35 \pm 20,65$ & $48,95 \pm 3,15$ & $43,74 \pm 4,89$ & $51,27 \pm 2,77$ & $44,98 \pm 1,74$ & $53,48 \pm 4,70$ & 6,27 & 0,15 & 0,53 \\
\hline Extensión Máxima Tobillo ( $\left.{ }^{\circ}\right)$ & $-16,12 \pm 3,56$ & $-7,37 \pm 1,20$ & $-20,66 \pm 6,36$ & $-19,23 \pm 5,64$ & $-15,99 \pm 8,42$ & $-20,17 \pm 14,31$ & $-18,71 \pm 3,59$ & $-15,50 \pm 6,45$ & 1,22 & 1,34 & 1,51 \\
\hline Extensión Máxima Tobillo (\%)* & $63,01 \pm 1,32$ & $74,80 \pm 4,70$ & $60,59 \pm 1,20$ & $76,29 \pm 2,26$ & $61,58 \pm 1,90$ & $77,77 \pm 2,07$ & $63,07 \pm 1,44$ & $77,81 \pm 1,15$ & 908,99 & 2,65 & 2,87 \\
\hline Flexión Tobillo Contacto Inicial ( $\left.{ }^{\circ}\right)$ & $3,45 \pm 5,16$ & $3,28 \pm 1,82$ & $4,77 \pm 3,91$ & $6,60 \pm 4,03$ & $5,39 \pm 3,64$ & $4,86 \pm 4,47$ & $2,66 \pm 2,44$ & $2,24 \pm 2,58$ & 0,16 & 1,55 & 2,13 \\
\hline
\end{tabular}
Hombres: H1 (<25\%), H2 (25\%-50\%), H3 (50\%-75\%), H4 (>75\%); Mujeres: M1 (<25\%), M2 (25\%-50\%), M3 (50\%-75\%), M4 (>75\%) 


\subsection{Comparativa de los patrones de la marcha reproducidos por el Exoesqueleto con el $30 \%$,} $50 \%$ y $70 \%$ de descarga de peso corporal y los patrones de la Marcha a velocidad Lenta

Se analizaron las diferencias de cada una de las variables entre el patrón de la marcha a velocidad lenta y el patrón reproducido por el exoesqueleto al 30\%, 50\% y $70 \%$ de descarga del peso corporal, de manera independiente para cada sexo. No fue posible analizar las variables de estudio del movimiento de la pelvis debido a la dificultad de adaptar el modelo de marcadores a la cesta pélvica del exoesqueleto.

\subsubsection{Parámetros espacio-temporales}

\subsubsection{Velocidad y cadencia}

La velocidad $(\mathrm{cm} / \mathrm{s})$, la velocidad normalizada en función de la estatura y la velocidad normalizada en función de la longitud de pierna en los hombres, mostraron valores menores en el $50 \%$ de descarga de peso que en la marcha lenta $(p=0,001)$. No se mostraron diferencias entre la marcha lenta y el $30 \%(p=0,055)$ y $70 \%$ de descarga $(p=0,171)$. Tampoco se presentaron diferencias entre los porcentajes de descarga de peso ( $p=0,171$ para $30 \%-50 \%$; $\mathrm{p}=0,584$ para $30 \%-70 \%$; $\mathrm{p}=0,055$ para $50 \%-70 \%$ ). La cadencia de los hombres (pasos $/ \mathrm{min}$ ) disminuyó aproximadamente un 30\% (de 42 pasos $/ \mathrm{min}$ a 30 pasos $/ \mathrm{min}$ ) en el 30\% ( $p=0,007)$, $50 \%(p=0,027)$ y $70 \%$ de descarga de peso $(p=0,014)$ en comparación con la marcha lenta. No se presentaron diferencias entre el $30 \%$ y $50 \%$ de descarga $(p=0,624)$, entre el $30 \%$ y $70 \%$ de descarga $(p=0,806)$, y entre el $50 \%$ y $70 \%$ de descarga de peso corporal $(p=0,806)$ (Tabla 26$)$. Las mujeres mostraron valores menores en el $30 \%(p=0,003)$ y el $50 \%(p=0,001)$ que en la marcha lenta en la velocidad $(\mathrm{cm} / \mathrm{s})$, velocidad normalizada en función de la estatura y la velocidad normalizada en función de la longitud de pierna. No se presentaron diferencias entre el $70 \%$ y la marcha lenta $(p=0,221)$, ni entre los diferentes porcentajes de descarga de peso ( $p=0,806$ para $30 \%-50 \% ; p=0,086$ para $30 \%-70 \% ; p=0,050$ para $50 \%-70 \%$ ). La cadencia en las mujeres disminuyó un $25 \%$, en el $30 \%(p=0,007), 50 \%(p=0,014)$ y $70 \%(p=0,027)$ de descarga comparando con la marcha lenta. No se mostraron diferencias entre el 30\% y 50\% $(p=0,806)$, el $30 \%$ y $70 \%(p=0,624)$, y el $50 \%$ y $70 \%(p=0,806)$ de descarga de peso corporal (Tabla 26$)$. 
Tabla 26. Parámetros Espacio-temporales: Velocidad y Cadencia (Velocidad Lenta y Porcentajes de Descarga con Exoesqueleto)

\begin{tabular}{|c|c|c|c|c|c|c|c|c|c|}
\hline \multicolumn{10}{|c|}{ Hombres } \\
\hline & \multicolumn{2}{|c|}{$30 \% \mathrm{DPC}$} & \multicolumn{2}{|c|}{$50 \%$ DPC } & \multicolumn{2}{|c|}{$70 \% \mathrm{DPC}$} & \multicolumn{2}{|c|}{ Marcha Lenta } & \multirow{2}{*}{$\chi^{2}(3)$} \\
\hline & Media \pm DS & IC $95 \%$ & Media \pm DS & IC $95 \%$ & Media \pm DS & IC $95 \%$ & Media \pm DS & IC $95 \%$ & \\
\hline Velocidad $(\mathrm{cm} / \mathrm{s})^{*}$ & $7,73 \pm 0,64$ & $6,71-8,76$ & $7,16 \pm 1,08$ & $5,44-8,88$ & $8,11 \pm 1,05$ & $6,44-9,78$ & $26,18 \pm 2,25$ & $22,60-29,76$ & 11,10 \\
\hline Velocidad/Estatura* & $0,04 \pm 0,01$ & $0,04-0,05$ & $0,04 \pm 0,01$ & $0,03-0,05$ & $0,05 \pm 0,01$ & $0,04-0,06$ & $0,15 \pm 0,01$ & $0,13-0,17$ & 11,10 \\
\hline Velocidad/Long. Pierna* & $0,09 \pm 0,01$ & $0,07-0,11$ & $0,08 \pm 0,01$ & $0,06-0,10$ & $0,09 \pm 0,02$ & $0,07-0,11$ & $0,30 \pm 0,03$ & $0,24-0,35$ & 11,10 \\
\hline \multirow[t]{4}{*}{ Cadencia (pasos/min)* } & $30,23 \pm 0,41$ & $29,73-30,74$ & $30,33 \pm 0,56$ & $29,63-31,02$ & $30,09 \pm 0,76$ & $29,15-31,03$ & $42,44 \pm 9,13$ & $31,10-53,77$ & 9,24 \\
\hline & \multicolumn{8}{|c|}{ Mujeres } & \\
\hline & \multicolumn{2}{|c|}{$30 \%$ DPC } & \multicolumn{2}{|c|}{$50 \%$ DPC } & \multicolumn{2}{|c|}{$70 \%$ DPC } & \multicolumn{2}{|c|}{ Marcha Lenta } & $x^{2} 21$ \\
\hline & Media \pm DS & IC 95\% & Media \pm DS & IC 95\% & Media \pm DS & IC 95\% & Media \pm DS & IC 95\% & \\
\hline Velocidad $(\mathrm{cm} / \mathrm{s})^{*}$ & $7,64 \pm 1,21$ & $6,14-9,14$ & $7,18 \pm 1,26$ & $5,61-8,75$ & $8,62 \pm 1,71$ & $6,50-10,73$ & $24,87 \pm 1,89$ & $22,52-27,21$ & 13,56 \\
\hline Velocidad/Estatura* & $0,05 \pm 0,01$ & $0,04-0,06$ & $0,04 \pm 0,01$ & $0,03-0,05$ & $0,05 \pm 0,01$ & $0,04-0,07$ & $0,15 \pm 0,02$ & $0,13-0,17$ & 13,56 \\
\hline Velocidad/Long. Pierna* & $0,09 \pm 0,02$ & $0,07-0,11$ & $0,09 \pm 0,02$ & $0,06-0,11$ & $0,10 \pm 0,02$ & $0,07-0,13$ & $0,30 \pm 0,03$ & $0,26-0,33$ & 13,56 \\
\hline Cadencia (pasos/min)* & $30,20 \pm 0,40$ & $29,70-30,70$ & $30,63 \pm 1,01$ & $29,38-31,88$ & $30,82 \pm 0,64$ & $30,03-31,61$ & $39,69 \pm 5,27$ & $33,15-46,23$ & 9,24 \\
\hline
\end{tabular}

$D P C($ descarga peso corporal) 


\subsubsection{Distancias}

Los hombres presentaron valores de longitud de paso $(\mathrm{cm})$, longitud de paso normalizada en función de la estatura y longitud de paso normalizada en función de la longitud de pierna, mayores en la marcha lenta que durante el $50 \%$ de descarga de peso $(p=0,001)$. No se mostraron diferencias entre el $30 \%(p=0,100)$ y $70 \%(p=0,100)$ de descarga del peso corporal con el exoesqueleto y la marcha lenta. Tampoco se mostraron diferencias entre el $30 \%$ y el $50 \%(p=0,100)$, entre el $30 \%$ y el $70 \%(p=1,000)$, y entre el $50 \%$ y el $70 \%$ de descarga de peso $(p=0,100)$. La anchura de paso $(\mathrm{cm})$ y la anchura de paso normalizada en función del ancho de pelvis presentaron en los hombres valores menores durante el patrón lento que en el $50 \%$ $(p=0,003)$ y el $70 \%(p=0,001)$ de descarga de peso. No se mostraron diferencias entre el $30 \%$ y la marcha lenta $(p=0,142)$. Tampoco existieron diferencias entre los porcentajes de descarga de peso, entre el $30 \%$ y el $50 \%(p=0,086)$, entre el $30 \%$ y el $70 \%(p=0,050)$, y entre el $50 \%$ y el 70\% ( $p=0,806)$ (Tabla 27).

La longitud de paso $(\mathrm{cm})$, la longitud de paso normalizada en función de la estatura y la longitud de paso normalizada en función de longitud de pierna presentó, en las mujeres, valores menores en el $30 \%(p=0,007)$ y el $50 \%(p=0,001)$ de descarga que en el patrón lento. No se mostraron diferencias entre el $70 \%$ de descarga y el patrón lento $(p=0,221)$. Durante el $50 \%$ de descarga los valores de longitud de paso $(\mathrm{cm})$, longitud de paso normalizada en función de la estatura y longitud de paso normalizada en función de longitud de pierna fueron menores que en el $70 \%(p=0,027)$. No fueron detectadas diferencias entre el $30 \%$ y el $50 \%$ $(p=0,462)$, y entre el $30 \%$ y el $70 \%(p=0,142)$ de descarga de peso. Las mujeres mostraron un ancho de paso y un ancho de paso normalizado en función del ancho de pelvis menor en el $50 \%(p=0,014)$ y el $70 \%(p<0,001)$ de descarga de peso que en el patrón lento. No se presentaron diferencias entre el $30 \%$ de descarga y el patrón lento $(p=0,221)$. Además, los valores fueron menores en el $30 \%$ que en el $70 \%$ de descarga $(p=0,014)$, y no se encontraron diferencias entre el $30 \%$ y el $50 \%(p=0,221)$, y el $50 \%$ y el $70 \%(p=0,221)$ de descarga de peso (Tabla 27). 
Tabla 27. Parámetros Espacio-temporales: Distancias (Velocidad Lenta y Porcentajes de Descarga con Exoesqueleto)

\begin{tabular}{|c|c|c|c|c|c|c|c|c|c|}
\hline \multicolumn{10}{|c|}{ Hombres } \\
\hline & \multicolumn{2}{|c|}{$30 \%$ DPC } & \multicolumn{2}{|c|}{$50 \%$ DPC } & \multicolumn{2}{|c|}{$70 \%$ DPC } & \multicolumn{2}{|c|}{ Marcha Lenta } & \multirow{2}{*}{$\chi^{2}(3)$} \\
\hline & Media \pm DS & IC $95 \%$ & Media \pm DS & IC 95\% & Media \pm DS & IC $95 \%$ & Media \pm DS & IC $95 \%$ & \\
\hline Longitud Paso $(\mathrm{cm})^{*}$ & $15,42 \pm 1,21$ & $13,50-17,35$ & $14,13 \pm 2,20$ & $10,64-17,63$ & $16,14 \pm 1,86$ & $13,19-19,10$ & $37,90 \pm 5,85$ & $28,60-47,21$ & 10,80 \\
\hline Long. Paso/Estatura* & $0,09 \pm 0,01$ & $0,07-0,10$ & $0,06 \pm 0,02$ & $0,03-0,10$ & $0,09 \pm 0,01$ & $0,07-0,11$ & $0,22 \pm 0,04$ & $0,16-0,27$ & 10,80 \\
\hline Long. Paso/Long. Pierna* & $0,18 \pm 0,02$ & $0,14-0,21$ & $0,13 \pm 0,04$ & $0,06-0,20$ & $0,18 \pm 0,03$ & $0,14-0,22$ & $0,43 \pm 0,07$ & $0,31-0,55$ & 10,80 \\
\hline Anchura Paso $(\mathrm{cm})^{*}$ & $32,59 \pm 6,26$ & $24,82-40,37$ & $37,77 \pm 2,85$ & $34,23-41,31$ & $38,81 \pm 3,12$ & $34,43-42,19$ & $14,16 \pm 1,50$ & $12,30-16,03$ & 13,56 \\
\hline Anchura Paso/Anchura Pelvis* & $1,35 \pm 0,20$ & $1,11-1,60$ & $1,58 \pm 0,10$ & $1,45-1,70$ & $1,60 \pm 0,18$ & $1,39-1,82$ & $0,59 \pm 0,08$ & $0,49-0,70$ & 11,88 \\
\hline \multicolumn{10}{|c|}{ Mujeres } \\
\hline & \multicolumn{2}{|c|}{$30 \%$ DPC } & \multicolumn{2}{|c|}{$50 \%$ DPC } & \multicolumn{2}{|c|}{$70 \%$ DPC } & \multicolumn{2}{|c|}{ Marcha Lenta } & \\
\hline & Media \pm DS & IC $95 \%$ & Media \pm DS & IC $95 \%$ & Media \pm DS & IC $95 \%$ & Media \pm DS & IC $95 \%$ & \\
\hline Longitud Paso $(\mathrm{cm})^{*}$ & $15,19 \pm 2,48$ & $12,11-18,27$ & $14,09 \pm 2,68$ & $10,76-17,43$ & $16,77 \pm 3,36$ & $12,59-20,94$ & $37,84 \pm 5,89$ & $30,53-45,16$ & 14,04 \\
\hline Long. Paso/Estatura* & $0,09 \pm 0,02$ & $0,07-0,11$ & $0,09 \pm 0,02$ & $0,06-0,11$ & $0,10 \pm 0,02$ & $0,07-0,13$ & $0,23 \pm 0,04$ & $0,19-0,27$ & 14,04 \\
\hline Long. Paso/Long. Pierna* & $0,18 \pm 0,03$ & $0,14-0,22$ & $0,17 \pm 0,04$ & $0,12-0,21$ & $0,20 \pm 0,04$ & $0,14-0,25$ & $0,45 \pm 0,07$ & $0,36-0,54$ & 14,04 \\
\hline Anchura Paso $(\mathrm{cm})^{*}$ & $25,61 \pm 7,62$ & $16,15-35,07$ & $31,62 \pm 4,43$ & $26,12-37,12$ & $33,98 \pm 2,96$ & $30,30-37,66$ & $13,45 \pm 2,00$ & $10,97-15,94$ & 15,00 \\
\hline Anchura Paso/Anchura Pelvis* & $1,17 \pm 0,30$ & $0,80-1,55$ & $1,46 \pm 0,17$ & $1,25-1,67$ & $1,57 \pm 0,11$ & $1,43-1,71$ & $0,63 \pm 0,12$ & $0,48-0,78$ & 15,00 \\
\hline
\end{tabular}

$D P C$ (descarga peso corporal) 


\subsubsection{Tiempos}

El tiempo de paso y de zancada de los hombres presentó valores mayores en el $30 \%$ ( $p=0,007$ para tiempo de paso; $p=0,003$ para tiempo de zancada) y $70 \%$ ( $p=0,007$ para tiempo de paso; $p=0,014$ para tiempo de zancada) de descarga de peso que en el patrón lento. No se mostraron diferencias significativas entre el $50 \%$ de descarga de peso y el patrón lento $(p=0,050)$ en el tiempo de paso y de zancada. Tampoco se presentaron diferencias entre el $30 \%$ y el $50 \%$ de descarga ( $p=0,462$ para tiempo de paso; $p=0,327$ para tiempo de zancada), entre el $30 \%$ y el $70 \%$ de descarga ( $p=1,000$ para tiempo de paso; $p=0,624$ para tiempo de zancada) y entre el $50 \%$ y el $70 \%$ de descarga ( $p=0,462$ para tiempo de paso; $p=0,624$ para tiempo de zancada). El tiempo de apoyo mostro valores mayores en el $30 \%(p<0,001)$ y el $50 \%(p=0,027)$ de descarga comparando con el patrón lento. No existieron diferencias en el tiempo de apoyo entre el $70 \%$ de descarga y el patrón lento $(p=0,086)$, y entre el $30 \%$ y el $50 \%(p=0,221)$, el $30 \%$ y el $70 \%$ $(p=0,086)$, y el $50 \%$ y el $70 \%$ de descarga de peso $(p=0,624)$. El tiempo de apoyo monopodal mostró valores mayores durante el $30 \%(p=0,006)$ y el $70 \%(p=0,028)$ de descarga de peso que en el patrón lento, no existiendo diferencias entre el $50 \%$ y el patrón lento $(p=0,100)$. Tampoco se presentaron diferencias entre los porcentajes de descarga en el tiempo de apoyo monopodal de los hombres, entre el $30 \%$ y el $50 \%(p=0,273)$, el $30 \%$ y el $70 \%(p=0,584)$, y el $50 \%$ y el $70 \%$ de descarga de peso $(p=0,584)$. En el tiempo de apoyo doble de los hombres se mostraron valores mayores en el $30 \%$ que en el patrón lento $(p=0,007)$ y en el $70 \%$ de descarga de peso $(p=0,027)$. No existieron diferencias entre el $50 \%(p=0,221)$ y el $70 \%(p=0,624)$ de descarga de peso y el patrón lento, entre el $30 \%$ y el $50 \%(p=0,142)$, y entre en $50 \%$ y el $70 \%$ de descarga $(p=0,462)$. El porcentaje del ciclo en apoyo $(p=0,106)$, y el porcentaje del ciclo en oscilación $(p=0,106)$ no mostraron ninguna diferencia entre los porcentajes de descarga y comparando con el patrón lento. Tampoco existieron diferencias en el tiempo de oscilación de los hombres $(p=0,118)$ (Tabla 28).

Las mujeres mostraron valores mayores de tiempo de paso, tiempo de zancada y tiempo de apoyo monopodal en el 30\% ( $p=0,014$ para tiempo de paso; $p=0,027$ para tiempo de zancada; $p=0,007$ para tiempo de apoyo monopodal), $50 \%(p=0,007$ para tiempo de paso; $p=0,014$ para tiempo de zancada; $p=0,027$ para tiempo de apoyo monopodal) y $70 \%$ ( $p=0,027$ para tiempo de paso; $p=0,007$ para tiempo de zancada; $p=0,014$ para tiempo de apoyo monopodal) de descarga de peso que en el patrón lento (Tabla 28). 
Tabla 28. Parámetros Espacio-temporales: Tiempos (Velocidad Lenta y Porcentajes de Descarga con Exoesqueleto)

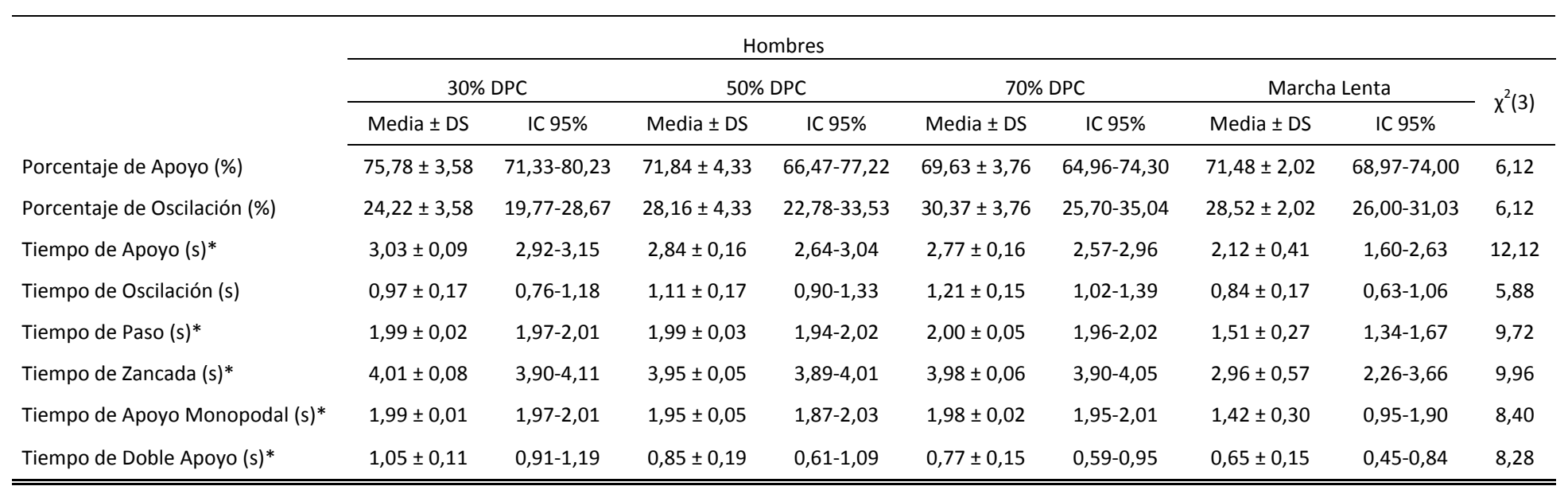

\begin{tabular}{|c|c|c|c|c|c|c|c|c|c|}
\hline \multicolumn{10}{|c|}{ Mujeres } \\
\hline & \multicolumn{2}{|c|}{$30 \%$ DPC } & \multicolumn{2}{|c|}{$50 \%$ DPC } & \multicolumn{2}{|c|}{$70 \%$ DPC } & \multicolumn{2}{|c|}{ Marcha Lenta } & \multirow{2}{*}{$\chi^{2}(3)$} \\
\hline & Media \pm DS & IC $95 \%$ & Media \pm DS & IC $95 \%$ & Media \pm DS & IC 95\% & Media \pm DS & IC $95 \%$ & \\
\hline Porcentaje de Apoyo (\%) & $71,07 \pm 5,50$ & $64,24-77,90$ & $69,77 \pm 6,36$ & $61,87-77,66$ & $67,69 \pm 4,89$ & $61,62-73,76$ & $75,45 \pm 2,50$ & $72,34-78,55$ & 7,80 \\
\hline Porcentaje de Oscilación (\%) & $28,93 \pm 5,50$ & $22,10-35,76$ & $30,23 \pm 6,36$ & $22,34-38,13$ & $32,31 \pm 4,89$ & $26,24-38,38$ & $24,55 \pm 2,50$ & $21,45-27,66$ & 7,80 \\
\hline Tiempo de Apoyo (s) & $2,82 \pm 0,22$ & $2,55-3,09$ & $2,74 \pm 0,27$ & $2,41-3,08$ & $2,68 \pm 0,21$ & $2,42-2,93$ & $2,32 \pm 0,31$ & $1,93-2,70$ & 5,40 \\
\hline Tiempo de Oscilación (s)* & $1,15 \pm 0,22$ & $0,87-1,42$ & $1,19 \pm 0,24$ & $0,88-1,49$ & $1,28 \pm 0,19$ & $1,05-1,51$ & $0,75 \pm 0,13$ & $0,60-0,91$ & 11,16 \\
\hline Tiempo de Paso (s)* & $1,99 \pm 0,03$ & $1,96-2,03$ & $1,98 \pm 0,08$ & $1,88-2,07$ & $1,97 \pm 0,03$ & $1,94-2,01$ & $1,54 \pm 0,21$ & $1,28-1,80$ & 9,24 \\
\hline Tiempo de Zancada $(\mathrm{s})^{*}$ & $3,96 \pm 0,03$ & $3,92-4,00$ & $3,93 \pm 0,06$ & $3,86-4,01$ & $3,95 \pm 0,05$ & $3,90-4,01$ & $3,07 \pm 0,40$ & $2,57-3,57$ & 9,24 \\
\hline Tiempo de Apoyo Monopodal (s)* & $1,97 \pm 0,01$ & $1,96-1,99$ & $1,95 \pm 0,05$ & $1,89-2,02$ & $1,98 \pm 0,03$ & $1,94-2,02$ & $1,53 \pm 0,19$ & $1,29-1,77$ & 9,24 \\
\hline Tiempo de Doble Apoyo (s) & $0,83 \pm 0,22$ & $0,56-1,09$ & $0,77 \pm 0,25$ & $0,45-1,08$ & $0,71 \pm 0,19$ & $0,48-0,94$ & $0,78 \pm 0,13$ & $0,61-0,94$ & 3,96 \\
\hline
\end{tabular}


No existieron diferencias entre el $30 \%$ y el $50 \%$ ( $p=0,806$ para tiempo de paso; $p=0,806$ para tiempo de zancada; $p=0,624$ para tiempo de apoyo monopodal), entre el $30 \%$ y el $70 \%$ $(\mathrm{p}=0,806$ para tiempo de paso; $\mathrm{p}=0,624$ para tiempo de zancada; $\mathrm{p}=0,806$ para tiempo de apoyo monopodal), y entre el $50 \%$ y el $70 \%$ ( $p=0,624$ para tiempo de paso; $p=0,806$ para tiempo de zancada; $p=0,806$ para tiempo de apoyo monopodal) de descarga de peso. El tiempo de oscilación presentó valores mayores en el $50 \%(p=0,014)$ y el $70 \%(p=0,001)$ de descarga en comparación con el patrón lento, no existiendo diferencias entre el $30 \%$ y el patrón lento $(p=0,086)$. No se mostraron diferencias entre el $30 \%$ y el $50 \%(p=0,462)$, entre el $30 \%$ y el $70 \%(p=0,142)$, y entre el $50 \%$ y el $70 \%(p=0,462)$ de descarga de peso en el tiempo de oscilación. El tiempo de apoyo $(p=0,145)$, el tiempo de doble apoyo $(p=0,266)$ y los porcentajes de apoyo $(p=0,050)$ y oscilación $(p=0,050)$ en el ciclo no mostraron diferencias en las mujeres (Tabla 28).

\subsubsection{Parámetros cinemáticos}

\subsubsection{Centro de gravedad}

El movimiento lateral del centro de gravedad en los hombres (plano frontal) se mostró mayor en el $50 \%(p=0,007)$ y el $70 \%(p=0,001)$ de descarga que durante el patrón lento, no habiendo diferencias significativas entre el $30 \%$ y el patrón lento $(p=0,221)$. Entre los porcentajes de descarga se presentaron valores menores en el $30 \%$ que en el $70 \%$ de descarga $(p=0,027)$, y no existieron diferencias entre el $30 \%$ y el $50 \%(p=0,142)$, y el $50 \%$ y el $70 \%$ de $(p=0,462)$ descarga de peso. El movimiento vertical del centro de gravedad (plano sagital) mostro valores mayores en el $30 \%(p=0,003)$, el $50 \%(p=0,014)$ y $70 \%(p=0,007)$ comparado con el patrón lento. No se presentaron diferencias entre los porcentajes de descarga, entre el $30 \%$ y el $50 \%(p=0,624)$, entre el $30 \%$ y el $70 \%(p=0,624)$, y entre el $50 \%$ y el $70 \%(p=0,327)$ de descarga de peso (Tabla 29).

El movimiento lateral del centro de gravedad en las mujeres (plano frontal) se mostró mayor en el $50 \%(p=0,014)$ y el $70 \%(p<0,001)$ de descarga que durante el patrón lento, no habiendo diferencias significativas entre el $30 \%$ y el patrón lento $(p=0,221)$. Entre los porcentajes de descarga se presentaron valores menores en el $30 \%$ que en el $70 \%$ de descarga $(p=0,014)$, y no existieron diferencias entre el $30 \%$ y el $50 \%(p=0,221)$, y el $50 \%$ y el $70 \%(p=0,221)$ de descarga de peso. El movimiento vertical del centro de gravedad (plano sagital) mostro valores mayores en el $30 \%(p=0,003)$, el $50 \%(p=0,014)$ y $70 \%(p=0,007)$ comparado con el patrón lento. No se presentaron diferencias entre los porcentajes de descarga, entre el $30 \%$ y el $50 \%(p=0,624)$, entre el $30 \%$ y el $70 \%(p=0,327)$, y entre el $50 \%$ y el $70 \%(p=0,324)$ de descarga de peso (Tabla 29). 
Tabla 29.Parámetros Cinemáticos: Centro de Gravedad (Velocidad Lenta y Porcentajes de Descarga con Exoesqueleto)

\begin{tabular}{|c|c|c|c|c|c|c|c|c|c|}
\hline \multicolumn{10}{|c|}{ Hombres } \\
\hline & \multicolumn{2}{|c|}{$30 \%$ DPC } & \multicolumn{2}{|c|}{$50 \% \mathrm{DPC}$} & \multicolumn{2}{|c|}{$70 \% \mathrm{DPC}$} & \multicolumn{2}{|c|}{ Marcha Lenta } & \multirow{2}{*}{$x^{2}(3)$} \\
\hline & Media \pm DS & IC $95 \%$ & Media \pm DS & IC $95 \%$ & Media \pm DS & IC $95 \%$ & Media \pm DS & IC $95 \%$ & \\
\hline CG plano frontal $(\mathrm{mm})^{*}$ & $658,96 \pm 119,70$ & $510,33-807,59$ & $764,84 \pm 53,08$ & $698,93-830,76$ & $785,10 \pm 57,30$ & $713,95-856,25$ & $143,54 \pm 20,55$ & $118,03-169,06$ & 14,04 \\
\hline CG plano sagital $(\mathrm{mm})^{*}$ & $126,91 \pm 17,32$ & $105,41-148,42$ & $133,29 \pm 9,19$ & $121,88-144,71$ & $129,33 \pm 6,51$ & $121,24-137,42$ & $17,71 \pm 4,14$ & $12,57-22,86$ & 9,96 \\
\hline \multicolumn{10}{|c|}{ Mujeres } \\
\hline & \multicolumn{2}{|c|}{$30 \%$ DPC } & \multicolumn{2}{|c|}{$50 \% \mathrm{DPC}$} & \multicolumn{2}{|c|}{$70 \% \mathrm{DPC}$} & \multicolumn{2}{|c|}{ Marcha Lenta } & 2 \\
\hline & Media \pm DS & IC $95 \%$ & Media \pm DS & IC $95 \%$ & Media \pm DS & IC $95 \%$ & Media \pm DS & IC 95\% & \\
\hline CG plano frontal $(\mathrm{mm})^{*}$ & $548,40 \pm 126,58$ & $391,23-705,58$ & $660,24 \pm 95,35$ & $541,86-778,63$ & $707,43 \pm 75,71$ & $613,42-801,44$ & $133,48 \pm 10,73$ & $120,17-146,80$ & 15,00 \\
\hline CG plano sagital $(\mathrm{mm})^{*}$ & $121,50 \pm 29,29$ & $85,14-157,87$ & $122,68 \pm 15,94$ & $102,90-142,47$ & $124,10 \pm 14,55$ & $106,04-142,17$ & $17,13 \pm 2,91$ & $13,52-20,75$ & 9,96 \\
\hline
\end{tabular}




\subsubsection{Cadera}

En el 30\% de descarga, los hombres obtuvieron el máximo de flexión de cadera (\%) en un instante posterior al patrón lento $(p=0,007)$, al $50 \%(p=0,014)$ y al $70 \%$ de descarga $(p=0,027)$. No existieron diferencias en el instante de flexión máxima de cadera en los hombres entre el $50 \%$ y $70 \%$ de descarga $(p=0,806)$, y entre el patrón lento y el $50 \%(p=0,806)$ y el $70 \%(p=0,624)$ de descarga de peso. La extensión máxima de cadera $\left({ }^{\circ}\right)$ mostró valores menores en el $50 \%$ $(p=0,001)$ y el $70 \%(p=0,014)$ comparado con el patrón lento, no habiendo diferencias entre el $30 \%$ y el patrón lento $(p=0,086)$ y entre los porcentajes de descarga $(p=0,142$ para $30 \%-50 \%$; $p=0,462$ para $30 \%-70 \% ; p=0,462$ para $50 \%-70 \%)$. No existieron diferencias $(p=0,160)$ en la flexión máxima de cadera $\left({ }^{\circ}\right)$, en el instante en el que sucede la extensión máxima de cadera (\%) $(p=0,323)$ ni tampoco en la flexión de cadera en el contacto inicial $\left({ }^{\circ}\right)$ en los hombres $(p=0,095)$ (Tabla 30).

Las mujeres mostraron un máximo de flexión de cadera $\left({ }^{\circ}\right)$ mayor en el patrón lento que durante el $30 \%(p=0,007)$, el $50 \%(p=0,027)$ y el $70 \%(p=0,014)$ de descarga de peso. No existieron diferencias entre el $30 \%$ y $50 \%(p=0,624)$, entre el $30 \%$ y $70 \%(p=0,806)$, y entre el $50 \%$ y $70 \%(p=0,806)$ de descarga de peso. Los valores de extensión máxima $\left({ }^{\circ}\right)$ fueron menores (mayor extensión) en el $50 \%(p=0,001)$ y $70 \%(p=0,027)$ de descarga que durante el patrón lento. No existieron diferencias entre el $30 \%$ y el patrón lento $(p=0,050)$, entre el $30 \%$ y $50 \%(p=0,221)$, entre el $30 \%$ y $70 \%(p=0,806)$, y entre el $50 \%$ y $70 \%(p=0,327)$ de descarga de peso. El instante del ciclo en el que sucede la extensión máxima de cadera (\%) se encontró posterior en el patrón lento que en el $50 \%(p=0,007)$ y $70 \%(p=0,007)$ de descarga de peso de las mujeres. No se encontraron diferencias en el instante del ciclo en el que sucede la máxima extensión de cadera en las mujeres entre el $30 \%$ y el patrón lento $(p=0,327)$, entre el $30 \%$ y $50 \%(p=0,086)$, entre el $30 \%$ y $70 \%(p=0,086)$, y entre el $50 \%$ y $70 \%(p=1,000)$ de descarga de peso. La flexión de cadera en el contacto inicial mostró valores menores durante el $50 \%$ $(p=0,014)$ y el $70 \%(p=0,001)$ de descarga que en el patrón lento, no habiendo diferencias entre el $30 \%$ y el patrón lento $(p=0,086)$, entre el $30 \%$ y el $50 \%(p=0,462)$, entre el $30 \%$ y el $70 \%(p=0,142)$, y entre el $50 \%$ y el $70 \%(p=0,462)$. No se encontraron diferencias en el instante del ciclo en el que se encuentra la flexión máxima de cadera $(\%)$ en las mujeres $(p=0,724)$ (Tabla 30). 
Tabla 30. Parámetros Cinemáticos: Cadera (Velocidad Lenta y Porcentajes de Descarga con Exoesqueleto)

\begin{tabular}{|c|c|c|c|c|c|c|c|c|c|}
\hline \multicolumn{10}{|c|}{ Hombres } \\
\hline & \multicolumn{2}{|c|}{$30 \%$ DPC } & \multicolumn{2}{|c|}{$50 \%$ DPC } & \multicolumn{2}{|c|}{$70 \% \mathrm{DPC}$} & \multicolumn{2}{|c|}{ Marcha Lenta } & \multirow{2}{*}{$\chi^{2}(3)$} \\
\hline & Media \pm DS & IC 95\% & Media \pm DS & IC $95 \%$ & Media \pm DS & IC 95\% & Media \pm DS & IC $95 \%$ & \\
\hline Flexión Máxima Cadera $\left({ }^{\circ}\right)$ & $32,41 \pm 2,72$ & $29,03-35,78$ & $31,79 \pm 3,72$ & $27,17-36,42$ & $33,37 \pm 3,16$ & $29,44-37,29$ & $38,65 \pm 3,05$ & $34,86-42,44$ & 5,16 \\
\hline Flexión Máxima Cadera (\%)* & $95,27 \pm 2,86$ & $91,72-98,82$ & $91,88 \pm 1,47$ & $90,05-93,71$ & $91,23 \pm 3,29$ & $87,14-95,32$ & $90,92 \pm 2,55$ & $87,76-94,08$ & 9,24 \\
\hline Extensión Máxima Cadera $\left({ }^{\circ}\right)^{*}$ & $-0,42 \pm 2,10$ & $-3,03-2,19$ & $-2,47 \pm 1,54$ & $-4,38-(-0,56)$ & $-1,40 \pm 1,87$ & $-3,72-0,92$ & $4,89 \pm 2,74$ & $1,49-8,29$ & 11,16 \\
\hline Extensión Máxima Cadera (\%) & $56,13 \pm 2,20$ & $53,40-58,87$ & $56,89 \pm 2,70$ & $53,54-60,23$ & $52,56 \pm 3,63$ & $48,05-57,07$ & $56,85 \pm 3,78$ & $52,16-61,55$ & 3,48 \\
\hline \multirow[t]{4}{*}{ Flexión Cadera Contacto Inicial $\left({ }^{\circ}\right)$} & $28,91 \pm 2,86$ & $25,39-32,43$ & $29,95 \pm 5,76$ & $22,80-37,10$ & $27,47 \pm 2,48$ & $24,39-30,55$ & $34,50 \pm 4,48$ & $28,94-40,06$ & 6,36 \\
\hline & \multicolumn{6}{|c|}{ Mujeres } & & & \\
\hline & \multicolumn{2}{|c|}{$30 \%$ DPC } & \multicolumn{2}{|c|}{$50 \%$ DPC } & \multicolumn{2}{|c|}{$70 \%$ DPC } & \multicolumn{2}{|c|}{ Marcha Lenta } & \multirow{2}{*}{$\chi^{2}(3)$} \\
\hline & Media \pm DS & IC 95\% & Media \pm DS & IC $95 \%$ & Media \pm DS & IC $95 \%$ & Media \pm DS & IC $95 \%$ & \\
\hline Flexión Máxima Cadera $\left({ }^{\circ}\right)^{*}$ & $29,08 \pm 2,33$ & $26,18-31,97$ & $29,60 \pm 1,12$ & $28,22-30,99$ & $29,48 \pm 2,26$ & $26,67-32,28$ & $42,51 \pm 3,37$ & $38,33-46,70$ & 9,24 \\
\hline Flexión Máxima Cadera (\%) & $89,37 \pm 4,77$ & $83,45-95,29$ & $89,27 \pm 6,32$ & $81,42-97,12$ & $87,92 \pm 5,57$ & $81,00-94,84$ & $90,91 \pm 1,78$ & $88,71-93,12$ & 1,32 \\
\hline Extensión Máxima Cadera $\left({ }^{\circ}\right)^{*}$ & $-3,22 \pm 2,94$ & $-6,86-0,43$ & $-5,69 \pm 1,61$ & $-7,69-(-3,69)$ & $-5,32 \pm 1,95$ & $-7,74-(-2,90)$ & $10,44 \pm 4,60$ & $4,73-16,15$ & 10,68 \\
\hline Extensión Máxima Cadera (\%)* & $56,83 \pm 3,67$ & $52,27-61,40$ & $53,35 \pm 4,51$ & $47,75-58,96$ & $52,36 \pm 4,63$ & $46,61-58,11$ & $60,57 \pm 3,54$ & $56,17-64,96$ & 10,68 \\
\hline Flexión Cadera Contacto Inicial $\left({ }^{\circ}\right)^{*}$ & $24,35 \pm 4,65$ & $18,58-30,12$ & $23,20 \pm 2,96$ & $19,52-26,88$ & $21,27 \pm 2,48$ & $18,19-24,35$ & $37,85 \pm 3,83$ & $33,10-42,60$ & 11,16 \\
\hline
\end{tabular}




\subsubsection{Rodilla}

Los hombres presentaron valores mayores en la flexión máxima de rodilla $\left({ }^{\circ}\right)$ durante el $30 \%$ $(p=0,003), 50 \%(p=0,027)$ y $70 \%(p=0,027)$ de descarga en comparación con el patrón lento. No se mostraron diferencias entre el $30 \%$ y $50 \%(p=0,462)$, entre el $50 \%$ y $70 \%(p=0,462)$, y entre el $50 \%$ y $70 \%(p=1,000)$ de descarga de peso. El instante del ciclo en el que sucede la flexión máxima de rodilla (\%) se presentó anterior en el 30\% de descarga que durante el $70 \%$ $(p=0,014)$ y el patrón lento $(p=0,027)$, no habiendo diferencias entre el patrón lento y el $50 \%$ $(p=0,142)$ y $70 \%(p=0,806)$ de descarga, entre el $30 \%$ y el $50 \%(p=0,462)$, y entre el $50 \%$ y el $70 \%(p=0,086)$ de descarga de peso. Los valores en la extensión máxima de rodilla $\left({ }^{\circ}\right)$ se presentaron mayores (menor extensión) durante el 30\% ( $p=0,001)$ y $70 \%(p=0,027)$ de descarga en comparación con el patrón lento, no habiendo diferencias entre el 50\% y el patrón lento $(p=0,050)$. Tampoco se encontraron diferencias en la extensión máxima de rodilla entre el $30 \%$ y $50 \%(p=0,221)$, entre el $30 \%$ y $70 \%(p=0,327)$, y entre el $50 \%$ y el $70 \%(p=0,806)$ de descarga de peso en los hombres. En la flexión de rodilla en el contacto inicial $\left({ }^{\circ}\right)$ se mostraron valores mayores en el $30 \%$ de descarga de peso que durante el patrón lento $(p=0,001)$, y no existieron diferencias entre el $50 \%(p=0,055)$ y $70 \%(p=0,171)$ y el patrón lento, entre el $30 \%$ y $50 \%(p=0,171)$, entre el $30 \%$ y el $70 \%(p=0,055)$, y entre el $50 \%$ y $70 \%(p=0,584)$ de descarga de peso. No se mostraron diferencias en los hombres en el instante del ciclo en el que sucede la extensión máxima de rodilla ( $p=0,392)$ (Tabla 31).

Las mujeres mostraron valores mayores de flexión máxima de rodilla durante el $30 \%$ de descarga que en el patrón lento $(p=0,007)$. No se mostraron diferencias en la flexión máxima de rodilla entre el patrón lento y el $50 \%(p=0,050)$ y $70 \%(p=0,462)$ de descarga, entre el $30 \%$ y $50 \%(p=0,462)$, entre el $30 \%$ y $70 \%(p=0,050)$, y entre el $50 \%$ y el $70 \%(p=0,221)$ de descarga de peso. El instante del ciclo en el que sucede la extensión máxima de rodilla (\%) se muestra, en las mujeres, posterior durante el $30 \%$ al patrón lento $(p=0,027)$ y al $70 \%(p=0,014)$ de descarga de peso. No se muestran diferencias entre el patrón lento y el $50 \%(p=0,142)$ y $70 \%(p=0,806)$, entre el $30 \%$ y $50 \%(p=0,462)$, y entre el $50 \%$ y $70 \%(p=0,086)$ de descarga de peso. La flexión de rodilla en el contacto inicial $\left({ }^{\circ}\right)$ es mayor durante el $30 \%(p=0,001)$ y $50 \%(p=0,027)$ de descarga que en el patrón lento, no existiendo diferencias entre el $70 \%$ y el patrón lento $(p=0,050)$, entre el $30 \%$ y $50 \%(p=0,327)$, entre el $30 \%$ y $70 \%(p=0,221)$, y entre el $50 \%$ y $70 \%$ $(p=0,806)$ de descarga de peso. No se mostraron diferencias en las mujeres en el instante del ciclo en el que sucede la flexión máxima de rodilla $(\%)(p=0,062)$, ni en la extensión máxima de rodilla $\left({ }^{\circ}\right)(p=0,472)($ Tabla 31$)$. 
Tabla 31. Parámetros Cinemáticos: Rodilla (Velocidad Lenta y Porcentajes de Descarga con Exoesqueleto)

\begin{tabular}{|c|c|c|c|c|c|c|c|c|c|}
\hline \multicolumn{10}{|c|}{ Hombres } \\
\hline & \multicolumn{2}{|c|}{$30 \% \mathrm{DPC}$} & \multicolumn{2}{|c|}{$50 \%$ DPC } & \multicolumn{2}{|c|}{$70 \%$ DPC } & \multicolumn{2}{|c|}{ Marcha Lenta } & \multirow{2}{*}{$\chi^{2}(3)$} \\
\hline & Media \pm DS & IC 95\% & Media \pm DS & IC 95\% & Media \pm DS & IC 95\% & Media \pm DS & IC 95\% & \\
\hline Flexión Máxima Rodilla $\left({ }^{\circ}\right)^{*}$ & $71,50 \pm 4,13$ & $66,36-76,63$ & $69,88 \pm 4,06$ & $64,84-74,92$ & $69,63 \pm 5,30$ & $63,04-76,21$ & $58,39 \pm 2,22$ & $55,64-61,15$ & 9,72 \\
\hline Flexión Máxima Rodilla (\%)* & $85,54 \pm 2,61$ & $82,30-88,79$ & $82,85 \pm 3,95$ & $77,94-87,75$ & $78,70 \pm 5,76$ & $71,55-85,85$ & $80,41 \pm 0,97$ & $79,21-81,61$ & 8,28 \\
\hline Extensión Máxima Rodilla $\left({ }^{\circ}\right)^{*}$ & $18,95 \pm 1,51$ & $17,08-20,83$ & $17,94 \pm 1,64$ & $15,91-19,98$ & $18,33 \pm 3,51$ & $13,96-22,69$ & $8,32 \pm 3,56$ & $3,89-12,74$ & 10,68 \\
\hline Extensión Máxima Rodilla (\%) & $48,20 \pm 3,21$ & $44,21-52,19$ & $46,18 \pm 6,47$ & $38,14-54,21$ & $42,71 \pm 2,22$ & $39,96-45,47$ & $46,34 \pm 3,98$ & $41,40-51,29$ & 3,00 \\
\hline Flexión Rodilla Contacto Inicial $\left({ }^{\circ}\right)^{*}$ & $32,29 \pm 3,61$ & $26,55-38,04$ & $26,60 \pm 1,11$ & $24,84-28,36$ & $24,38 \pm 4,02$ & $17,98-30,78$ & $11,63 \pm 3,88$ & $5,46-17,80$ & 11,10 \\
\hline
\end{tabular}

\begin{tabular}{|c|c|c|c|c|c|c|c|c|}
\hline \multicolumn{8}{|c|}{ Mujeres } & \multirow{3}{*}{$x^{2}(3)$} \\
\hline \multicolumn{2}{|c|}{$30 \%$ DPC } & \multicolumn{2}{|c|}{$50 \%$ DPC } & \multicolumn{2}{|c|}{$70 \%$ DPC } & \multicolumn{2}{|c|}{ Marcha Lenta } & \\
\hline Media \pm DS & IC 95\% & Media \pm DS & IC 95\% & Media \pm DS & IC 95\% & Media \pm DS & IC 95\% & \\
\hline $68,23 \pm 4,02$ & $63,24-73,22$ & $67,00 \pm 4,04$ & $61,98-72,02$ & $65,35 \pm 4,16$ & $60,18-70,51$ & $59,24 \pm 4,18$ & $54,04-64,43$ & 8,76 \\
\hline $80,70 \pm 6,34$ & $72,82-88,57$ & $79,38 \pm 3,56$ & $74,96-83,79$ & $76,56 \pm 2,38$ & $73,60-79,51$ & $82,06 \pm 1,59$ & $80,08-84,03$ & 7,32 \\
\hline $15,91 \pm 2,57$ & $12,73-19,10$ & $14,88 \pm 2,99$ & $11,17-18,60$ & $15,52 \pm 3,88$ & $10,70-20,33$ & $10,55 \pm 5,21$ & $4,08-17,02$ & 2,52 \\
\hline $48,12 \pm 4,58$ & $42,44-53,80$ & $46,13 \pm 4,39$ & $40,68-51,58$ & $38,29 \pm 6,92$ & $29,70-46,88$ & $41,21 \pm 3,17$ & $37,27-45,14$ & 8,28 \\
\hline $28,86 \pm 4,36$ & $23,41-34,24$ & $24,17 \pm 2,36$ & $21,24-27,09$ & $23,10 \pm 3,46$ & $18,80-27,40$ & $10,55 \pm 2,51$ & $7,43-13,67$ & 10,68 \\
\hline
\end{tabular}




\subsubsection{Tobillo}

La flexión máxima de tobillo en los hombres $\left({ }^{\circ}\right)$ se presentó mayor durante el $30 \%$ de descarga que en el $50 \%(p=0,007)$ y en el patrón lento $(p=0,003)$, no habiendo diferencias entre el patrón lento y el $50 \%(p=0,806)$ y $70 \%(p=0,221)$ de descarga, entre el $30 \%$ y $70 \%(p=0,086)$, y entre el $50 \%$ y $70 \%(p=0,327)$ de descarga de peso. Los valores de extensión máxima de tobillo $\left({ }^{\circ}\right)$ fueron mayores (menor extensión) en el 30\% de descarga que en el 50\% ( $\left.p=0,027\right), 70 \%$ $(p=0,014)$ y el patrón lento $(p=0,001)$. No existieron diferencias entre el patrón lento y el $50 \%$ $(p=0,327)$ y $70 \%$ de descarga $(p=0,221)$, ni entre el $50 \%$ y $70 \%(p=0,806)$ de descarga en la extensión máxima de tobillo de los hombres. El instante del ciclo en el que sucede la extensión máxima de tobillo (\%) se presenta posterior en el 30\% de descarga comparando con el $70 \%$ $(p=0,014)$ y el patrón lento $(p=0,003)$, no habiendo diferencias entre el patrón lento y el $50 \%$ $(p=0,327)$ y $70 \%(p=0,324)$ de descarga, entre el $30 \%$ y $50 \%(p=0,050)$, ni entre el $50 \%$ y $70 \%$ $(p=0,624)$ de descarga de peso. La flexión de tobillo en el contacto inicial $\left({ }^{\circ}\right)$ fue mayor en el $30 \%$ de descarga que en el $70 \%(p=0,003)$ y el patrón lento $(p=0,014)$, y no se mostraron diferencias entre el patrón lento y el $50 \%(p=0,624)$ y $70 \%(p=0,624)$ de descarga, entre el $30 \%$ y $50 \%(p=0,050)$, ni entre el $50 \%$ y $70 \%(p=0,327)$ de descarga de peso. No existieron diferencias en los hombres en el instante en el que sucede la flexión máxima de tobillo (\%) $(p=0,516)($ Tabla 32).

Las mujeres mostraron el instante del ciclo en el que sucede la flexión máxima de tobillo (\%) anterior durante el $70 \%$ de descarga que el patrón lento $(p=0,027)$ y el $30 \%(p=0,007)$, y no existieron diferencias entre el patrón lento y el $30 \%(p=0,624)$ y $50 \%(p=0,462)$ de descarga de peso, entre el $30 \%$ y $50 \%(p=0,221)$, ni entre el $50 \%$ y $70 \%(p=0,142)$. Los valores de extensión máxima de tobillo $\left({ }^{\circ}\right.$ ), en las mujeres, fueron mayores (menor extensión) durante el $30 \%$ de descarga que en el patrón lento $(p=0,007)$ y el $70 \%(p=0,014)$, no habiendo diferencias entre el patrón lento y el $50 \%(p=0,142)$ y $70 \%(p=0,806)$ de descarga, entre el $30 \%$ y $50 \%(p=0,221)$, y entre el $50 \%$ y $70 \%(p=0,221)$ de descarga de peso corporal. No existieron diferencias $(p=0,062)$ en las mujeres en la flexión máxima de tobillo $\left({ }^{\circ}\right)$, en la flexión de tobillo durante el contacto inicial $\left(^{\circ}\right)(p=0,050)$, ni en el instante del ciclo en el que sucede la extensión máxima de tobillo $(\%)(p=0,118)$ (Tabla 32). 
Tabla 32. Parámetros Cinemáticos: Tobillo (Velocidad Lenta y Porcentajes de Descarga con Exoesqueleto)

\begin{tabular}{|c|c|c|c|c|c|c|c|c|c|}
\hline \multicolumn{10}{|c|}{ Hombres } \\
\hline & Media \pm DS & IC $95 \%$ & Media \pm DS & IC $95 \%$ & Media \pm DS & IC $95 \%$ & Media \pm DS & IC $95 \%$ & $\chi^{2}(3)$ \\
\hline Flexión Máxima Tobillo (\%) & $49,76 \pm 3,84$ & $44,99-54,52$ & $47,60 \pm 13,60$ & $30,71-64,49$ & $46,94 \pm 5,12$ & $40,59-53,30$ & $51,93 \pm 3,90$ & $47,09-56,78$ & 2,28 \\
\hline Extensión Máxima Tobillo $\left({ }^{\circ}\right)^{*}$ & $0,59 \pm 2,98$ & $-3,11-4,29$ & $-5,18 \pm 5,22$ & $-11,66-1,29$ & $-6,29 \pm 5,57$ & $-13,20-0,62$ & $-11,40 \pm 7,21$ & $-20,35-(-2,44)$ & 10,68 \\
\hline \multicolumn{10}{|c|}{ Mujeres } \\
\hline & \multicolumn{2}{|c|}{$30 \%$ DPC } & \multicolumn{2}{|c|}{$50 \%$ DPC } & \multicolumn{2}{|c|}{$70 \%$ DPC } & \multicolumn{2}{|c|}{ Marcha Lenta } & \multirow{2}{*}{$\chi^{2}(3)$} \\
\hline & Media \pm DS & IC $95 \%$ & Media \pm DS & IC $95 \%$ & Media \pm DS & IC $95 \%$ & Media \pm DS & IC $95 \%$ & \\
\hline Flexión Máxima Tobillo ( ${ }^{\circ}$ ) & $18,70 \pm 3,06$ & $14,89-22,50$ & $16,30 \pm 2,70$ & $12,95-19,65$ & $11,39 \pm 2,86$ & $7,83-14,94$ & $17,21 \pm 3,20$ & $13,24-21,19$ & 7,32 \\
\hline Flexión Tobillo Contacto Inicial ( ${ }^{\circ}$ ) & $7,83 \pm 3,12$ & $3,95-11,71$ & $4,95 \pm 3,71$ & $0,34-9,56$ & $2,25 \pm 2,64$ & $-1,03-5,53$ & $5,14 \pm 3,05$ & $1,35-8,93$ & 7,80 \\
\hline
\end{tabular}



5. Discusión 

La presente investigación trata de examinar la idoneidad del patrón reproducido por el sistema Hybrid y la adecuación de éste para los procesos de rehabilitación y compensación de la marcha. Los resultados obtenidos ponen de manifiesto el cumplimiento de las hipótesis formuladas en el presente estudio, 1) existiendo diferentes patrones en la marcha a velocidad normal en función del sexo y la estatura de los sujetos, 2) mostrando diferencias entre el patrón de la marcha a velocidad normal y el patrón de la marcha a la velocidad lenta y, 3) señalando las modificaciones en el patrón reproducido por el HYBRID a causa de la descarga de peso corporal, con lo que podría no considerarse idóneo para la aplicación en procesos de rehabilitación.

Durante la discusión se describirá cómo se ha logrado la consecución de los objetivos establecidos durante la investigación de forma secuencial en el orden en el que se plantearon en la introducción de la tesis.

\subsection{Análisis de las diferencias en la Marcha Normal en función del sexo y de la estatura de} los sujetos

La descripción de la marcha normal se presenta imprescindible para la comprensión de los patrones patológicos, ya que proporciona el estándar con que el cada sujeto será evaluado. El término "normal", como ya se ha mencionado, implica ambos sexos y una variedad de características antropométricas que condicionan y caracterizan el patrón de la marcha (Whittle, 2007). Por ello, uno de los objetivos del presente estudio fue hallar las diferencias en el patrón de la marcha normal en función del sexo y en función de la estatura. Identificando así, patrones independientes por grupos de estatura para hombres y mujeres.

Las principales diferencias en el patrón de la marcha normal, a velocidad libremente seleccionada, se encuentran básicamente entre hombres y mujeres. Los parámetros temporales muestran un menor tiempo de paso y de zancada en las mujeres, señalado también por Cho et al. (2004), debido al menor tiempo de oscilación en las mujeres y, por tanto, al menor tiempo de apoyo monopodal, y a un mayor tiempo de doble apoyo. Este hecho se traduce en un menor, aunque no significativo, porcentaje de oscilación y mayor porcentaje de apoyo en las mujeres. Cuando los parámetros espaciales son analizados, se muestra que los valores de velocidad, longitud de paso y longitud de zancada sin normalizar no difieren entre hombres y mujeres. Por ello las mujeres, con estaturas y longitudes de pierna menores, presentan valores de velocidad, longitud de paso y longitud de zancada normalizadas proporcionalmente mayores que los hombres. Dichos resultados de velocidad normalizada son contrarios a los descritos por Cho et al. (2004), Crosbie et al. (1997) y Kerrigan et al. (1998), y estarían relacionados con una mayor longitud de paso y longitud de zancada normalizadas (en 
función de la estatura y en función de la longitud de pierna), como describen Frimenko et al. (2015), y una mayor cadencia de paso. Aunque otros autores han atribuido las mayores longitudes de paso y mayores cadencias a los hombres (Chiu et al., 2013; Cho et al., 2004; Kerrigan et al., 1998), los estudios realizados por Bruening et al. (2015), Crosbie et al. (1997) y Frimenko et al. (2015) presentan los mismos valores de cadencia para las mujeres que en el presente estudio.

Si bien los parámetros espacio-temporales muestran algunas diferencias entre hombres y mujeres, los parámetros cinemáticos podrían considerarse las características más diferenciadoras entre sexos. La cadera, de forma similar a los resultados descritos por Cho et al. (2004), se presenta en las mujeres con un mayor grado de flexión que en los hombres durante el ciclo de la marcha, alcanzando una mayor flexión máxima en la fase final de oscilación, mayor flexión en el instante del contacto inicial, y menor extensión alrededor del $50 \%$ del ciclo (Figura 49). Esta flexión de cadera repercute y se encuentra íntimamente relacionada con el comportamiento de la rodilla. Las mujeres también presentan mayor flexión de rodilla en el contacto inicial, como señala Nigg et al. (1994), y mayor flexión máxima en el instante del despegue del pie y menor extensión que los hombres (Røislien et al., 2009) (Figura $50)$.

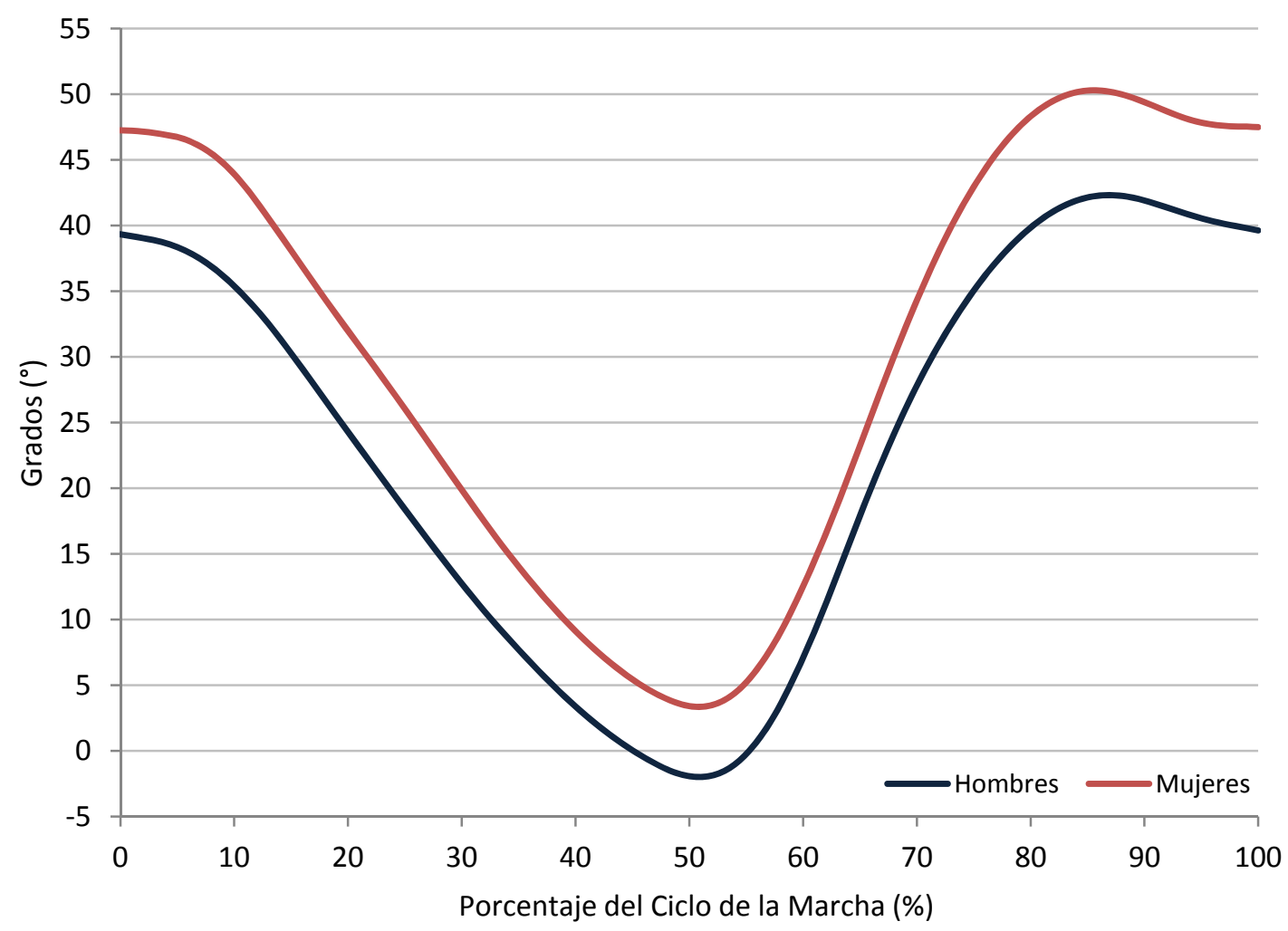

Figura 49. Cinemática de la cadera. Diferencias entre el patrón de hombres y mujeres 


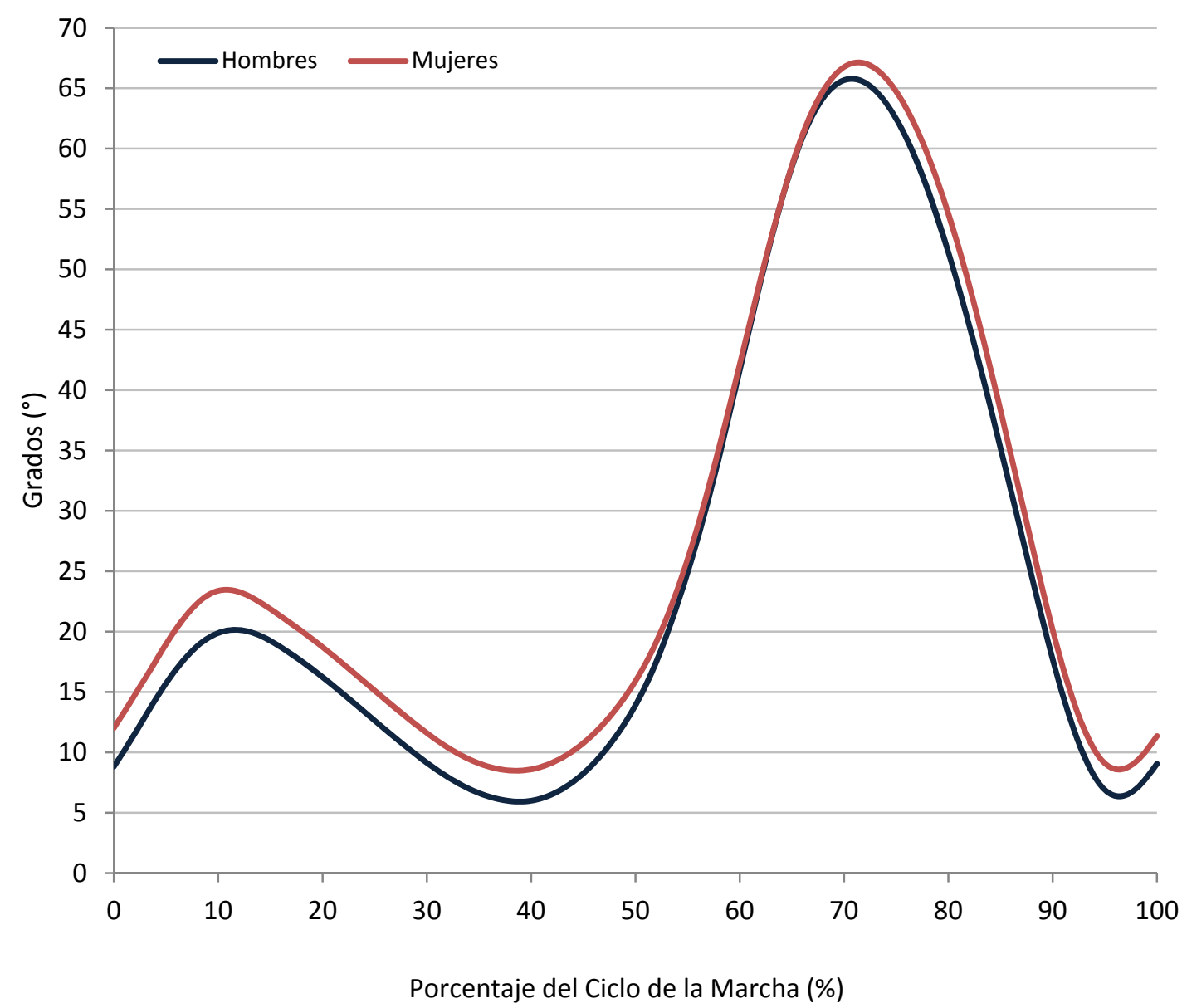

Figura 50. Cinemática de la rodilla. Diferencias entre el patrón de hombres y mujeres.

El tobillo, influenciado por el patrón cinemático de la cadera, describe en las mujeres una mayor extensión (flexión plantar) en el instante previo al despegue del pie (Bruening et al., 2015; Kerrigan et al., 1998; Røislien et al., 2009). Este hecho aparecería como consecuencia de la menor extensión de cadera de las mujeres y podría explicarse como forma de compensación en el instante previo al despegue del pie y empuje de la pierna que comienza la fase de oscilación. Las mujeres, con pies proporcionalmente más pequeños que los hombres, necesitarían, además, incrementar la extensión del tobillo en el despegue del pie para conservar la cinemática de la cadera y la rodilla durante la fase de oscilación (Figura 51). 


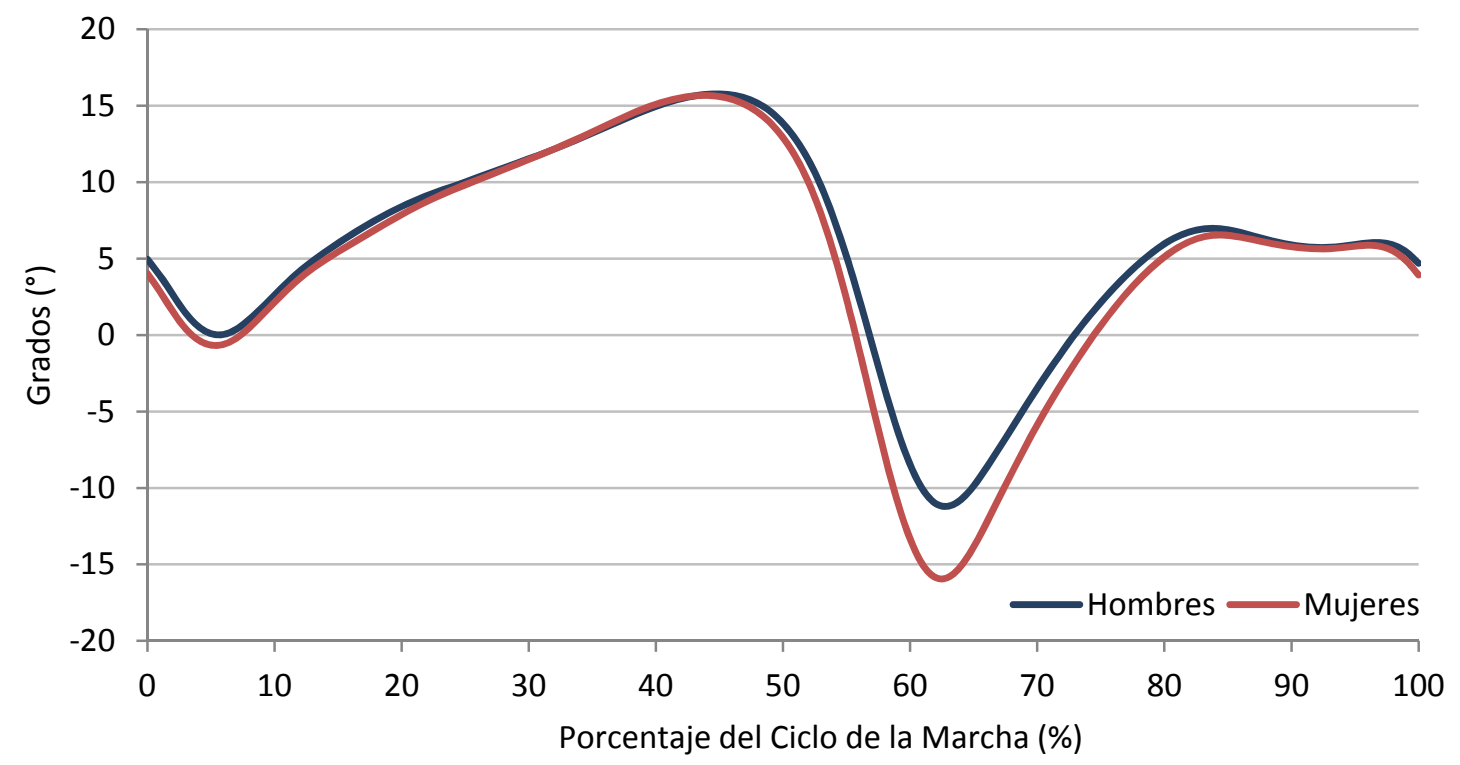

Figura 51. Cinemática del tobillo. Diferencias entre el patrón de hombres y mujeres.

Las mujeres presentan mayor basculación lateral de la pelvis $\left(15,47 \pm 3,34^{\circ}\right.$ para mujeres; $11,81 \pm 3,39^{\circ}$ para hombres), mientras que los hombres realizan un movimiento lateral de mayor amplitud (de lado a lado) del centro de gravedad $(61,99 \pm 11,24 \mathrm{~mm}$ para hombres; $48,90 \pm 10,34 \mathrm{~mm}$ para mujeres). Estas diferencias en los movimientos de la pelvis y centro de gravedad en el plano frontal se han identificado como los mayores discriminantes entre los patrones de la marcha entre hombres y mujeres (Bruening et al., 2015; Mather, \& Murdoch, 1994; Murray et al., 1964; Murray et al., 1970; Smith et al., 2002; Troje, 2002). Algunos autores, plantean la hipótesis de que las diferencias en el movimiento de pelvis y centro de gravedad en el plano frontal entre hombres y mujeres puede deberse a una elección basada en aspectos socioculturales (Bruening et al., 2015), adaptaciones evolutivas (Troje, 2002), o, con mayor probabilidad, a una combinación de factores socio-culturales, anatómicos y biomecánicos.

Aunque las principales características diferenciadoras en el patrón de la marcha se muestran en relación al sexo, se encontraron algunos parámetros influenciados por la estatura de los sujetos. Los parámetros espacio-temporales no presentan diferencias relevantes que puedan atribuirse al efecto de la estatura, sino más bien a diferencias anecdóticas propias de las características individuales de los sujetos analizados. Pero, en cambio, se pone de manifiesto en estos parámetros la importancia de la normalización en función de las características antropométricas, estatura o longitud de pierna, para una mejor comprensión de los patrones (Hof, 1996; Pierrynowski, \& Galea, 2001). Parámetros como la longitud de paso y de zancada, que presentan valores menores en los hombres con estaturas más bajas, no muestran ninguna 
diferencia una vez son normalizadas en función de la estatura o de la longitud de paso. Hecho que también hace señalar la flexibilidad que existe en la elección del parámetro antropométrico, estatura o longitud de pierna, para la normalización. Ya que ambas normalizaciones, en hombres y en mujeres, presentan las mismas similitudes entre grupos de estatura.

En cambio, los parámetros cinemáticos muestran algunas diferencias reseñables entre los grupos de estatura. La cinemática de la pelvis de las mujeres presenta entre $1^{\circ}$ y $1,5^{\circ}$ menor amplitud de movimiento (anteversión-retroversión) en el grupo que se encuentra por debajo del percentil 25 (M1) que en el resto de las mujeres. Esta diferencia estaría relacionada con la extensión de cadera y la longitud de paso, que también se muestran menores en este grupo, aunque no significativamente. No podría determinarse si la menor amplitud de anteversiónretroversión de la pelvis se presenta como consecuencia del movimiento de la cadera o viceversa, sino que debería considerarse como parte del patrón cinemático de las mujeres con estaturas menores que tiene como consecuencia longitudes de paso y zancada más cortas.

En los hombres se presenta una mayor extensión (flexión plantar) del tobillo, de aproximadamente $5^{\circ}$, en los sujetos pertenecientes a los grupos por debajo del percentil 50 $(\mathrm{H} 1$ y $\mathrm{H} 2)$ que en los de mayor estatura $(\mathrm{H} 3$ y $\mathrm{H} 4)$. Este hecho se explica de igual manera que la mayor extensión del tobillo de las mujeres en el instante del despegue del pie; sujetos con pies proporcionalmente menores podrían tener la necesidad de incrementar la extensión del tobillo durante la fase de despegue del pie para mantener la efectividad del patrón cinemático de cadera y rodilla durante la fase de oscilación (Bruening et al., 2015) (Figura 52).

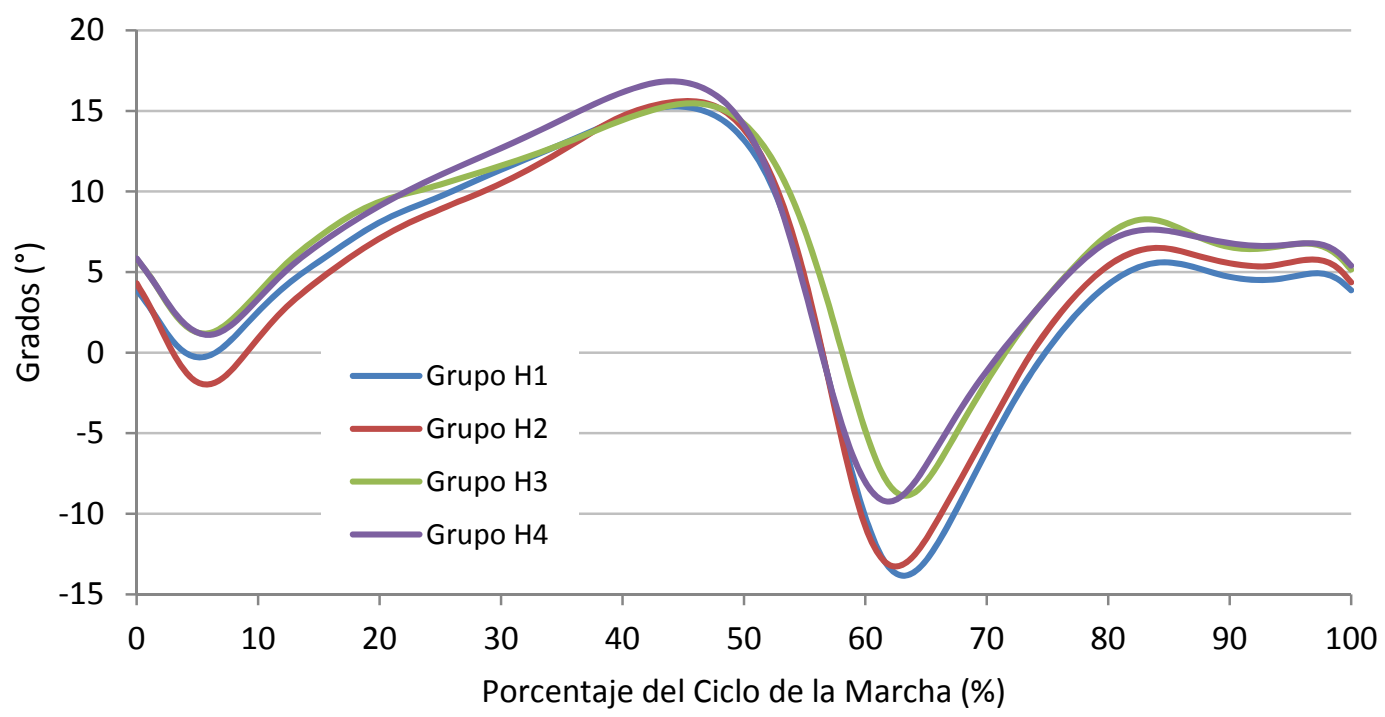

Figura 52. Cinemática del tobillo. Diferencias entre los grupos de estatura de los hombres. 
DISCUSIÓN

No podría cuestionarse la identificación de patrones de la marcha independientes para hombres y mujeres. Las desigualdades entre sexos en los parámetros espacio-temporales y, fundamentalmente, en los parámetros cinemáticos señalan la importancia del tratamiento diferenciado de los patrones para la evaluación y comparación con los estándares propios de cada sexo. Además, aunque menos evidentes, las diferencias y estrategias seleccionadas por los diferentes grupos de estatura no son despreciables y hacen señalar también la necesidad de establecer patrones para cada uno de los grupos de estatura. Sería razonable, por tanto, tener en consideración estas diferencias en el momento de la identificación de los patrones normales de la marcha. 


\subsection{Comparativa de los patrones de Marcha a velocidad Lenta $(0.25 \mathrm{~m} / \mathrm{s})$ y los patrones de la} Marcha a velocidad Normal

Una vez analizadas las diferencias en el patrón de la marcha a velocidad normal y habiendo establecido un patrón característico para cada sexo y grupo de estatura, en base a los objetivos de la presente investigación es pertinente analizar los cambios que se han producido, como consecuencia de la disminución de la velocidad (de $130 \mathrm{~cm} / \mathrm{s}$ a $25 \mathrm{~cm} / \mathrm{s}$ ), en hombres y mujeres por separado. Las diferencias encontradas en función del grupo entre la velocidad normal y la velocidad lenta no podrían atribuirse a la influencia de la estatura, sino que son el resultado de diferencias particulares y aisladas que no parecen seguir una tendencia que pueda identificarse y relacionarse con la estatura. Todos los sujetos de un mismo sexo, independientemente de su estatura, presentan un mismo patrón lento y muestran las mismas diferencias comparado con el patrón normal de la marcha. Por tanto, a continuación se desarrollarán y explicarán las adaptaciones que se han producido por hombres y mujeres en el patrón de la marcha para disminuir la velocidad hasta $25 \mathrm{~cm} / \mathrm{s}$.

Baker (2013), Bejek et al. (2006), Kirtley (2006), Shemmell et al. (2007), y Tanawongsuwan y Bobick (2003), determinaron que los parámetros que se encuentran directamente afectados por la disminución de la velocidad son la cadencia y longitud de paso, estableciendo una relación lineal directa con la velocidad. En el presente estudio la cadencia y longitud de paso se reducen aproximadamente un $65 \%$ y un $45 \%$ respectivamente. Tanto hombres como mujeres disminuyen la cadencia desde valores superiores a 110 pasos/min en la marcha normal a 40 pasos/min durante la marcha lenta, y reducen a la mitad la longitud de paso y de zancada. Pero son los parámetros temporales, más que los espaciales, los que muestran mayores modificaciones al reducir la velocidad de la marcha. Hombres y mujeres, en similar proporción, triplican el tiempo de paso y el tiempo de zancada durante la marcha lenta. El aumento del tiempo de paso se debe fundamentalmente al aumento del tiempo de apoyo, puesto que, aunque el tiempo de oscilación se incrementa en un $80 \%$, el tiempo de apoyo triplica su duración. Esto, a su vez, se relaciona con el gran aumento del tiempo de doble apoyo, en concordancia con Bejek et al. (2006) y Kirtley (2006), que septuplica su valor en el presente estudio. Todas estas modificaciones, similares en hombres y mujeres, repercuten en la relación de las fases de apoyo y oscilación, y en su distribución en el ciclo de la marcha (Baker, 2013; Bejek et al., 2006; Shemmell et al., 2007). Aumenta la proporción de fase de apoyo, que pasa de ser un $60 \%$ del ciclo de la marcha normal a ocupar más de un $70 \%$ del ciclo en la marcha lenta, y consecuentemente la proporción de fase de oscilación disminuye de un $40 \%$ a aproximadamente un $25 \%$ del ciclo. Esto significa que el despegue del pie se retrasa un $10 \%$ y que todos los eventos relativos a la cinemática de las articulaciones, que aparecen durante la 
fase de oscilación, sucederán durante un tiempo menor. Además, los máximos y mínimos de flexo-extensión de la cadera, rodilla y tobillo se retrasarán en el tiempo, apareciendo a partir del $45 \%$ del ciclo de la marcha.

La cadera, de hombres y mujeres, sufre las mismas adaptaciones en el patrón de flexoextensión a velocidad lenta. El mínimo de la cadera se retrasa del 50\% al $60 \%$ del ciclo aproximadamente y el máximo aparece ligeramente posterior, entre un $3 \%$ en hombres y un $5 \%$ en mujeres, encontrándose en torno al $90 \%$ del ciclo en la marcha a velocidad lenta. Además, aunque se mantiene la suavidad de la curva de la cinemática de la cadera en el plano sagital, se encuentra una gran disminución del rango de movimiento de manera proporcional para hombres y mujeres (Figuras 53 y 54), alcanzándose menor flexión y extensión de cadera en todo el ciclo de la marcha (Bejek et al., 2006; Riley et al., 2001).

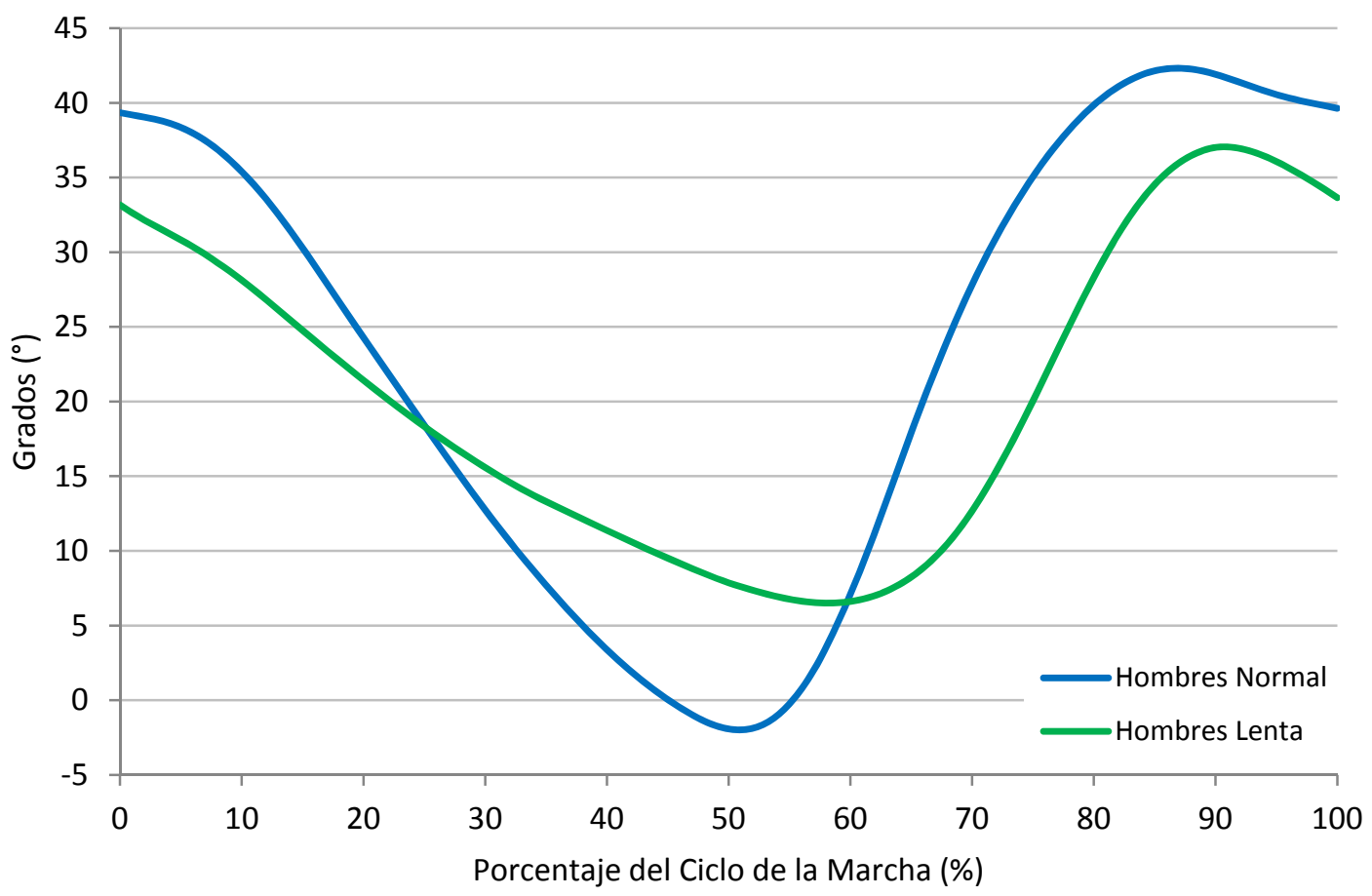

Figura 53. Cinemática de la cadera. Diferencias entre marcha normal y marcha lenta en hombres. 


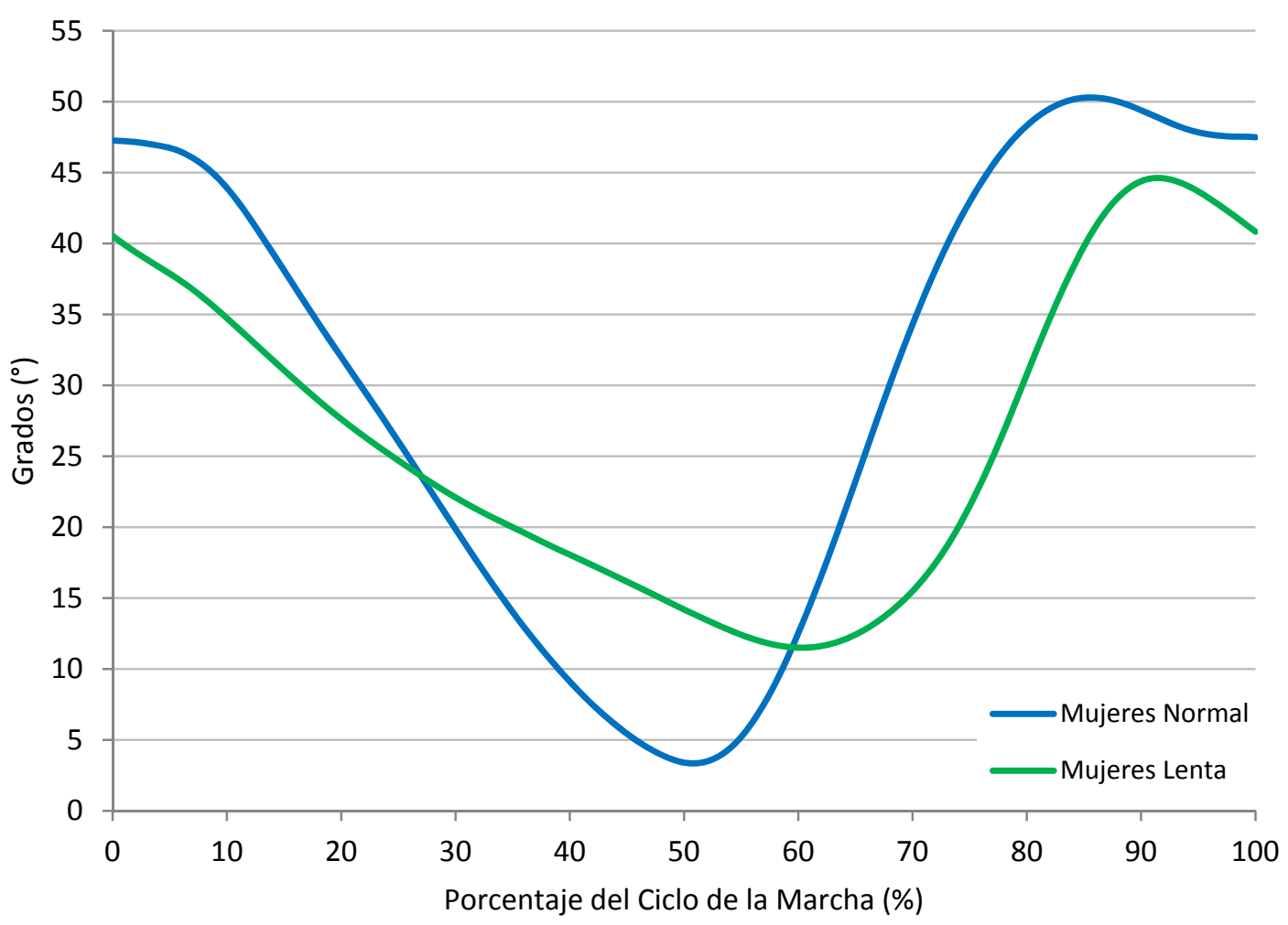

Figura 54. Cinemática de la cadera. Diferencias entre marcha normal y marcha lenta en mujeres.

En la rodilla, al igual que en la cadera, los máximos y los mínimos se retrasan en el ciclo alrededor de un $9-11 \%$ y un $5-6 \%$ respectivamente, tanto para los hombres como para las mujeres. Como señalan Baker (2013), Bejek et al. (2006) y Riley et al. (2001), la flexión máxima de rodilla disminuye cuando la velocidad se reduce, y se alcanza también una menor extensión durante la marcha lenta, disminuyendo así el rango de movimiento. En el presente estudio, tanto hombres como mujeres muestran estas modificaciones en el patrón de la marcha lenta, aun no siendo todas significativas (extensión de rodilla en mujeres). Además, la flexión de la rodilla en el contacto inicial disminuye en los hombres y aumenta en las mujeres, alcanzando en ambos sexos valores en torno a $10^{\circ}$ de flexión durante la marcha lenta. Otro hecho relevante, apreciable únicamente visualizando las gráficas del comportamiento cinemático de la rodilla en flexo-extensión, es la desaparición, en la velocidad lenta, del primer pico de flexión, en torno al 10\% del ciclo en ambos sexos. Dicha desaparición se corresponde con la amortiguación del peso corporal tras el contacto inicial (Kirtley, 2006). La rodilla mantiene prácticamente la misma posición desde el contacto inicial hasta el instante anterior al comienzo de la fase previa a la oscilación, en torno al 50\% del ciclo (Figuras 55 y 56). 


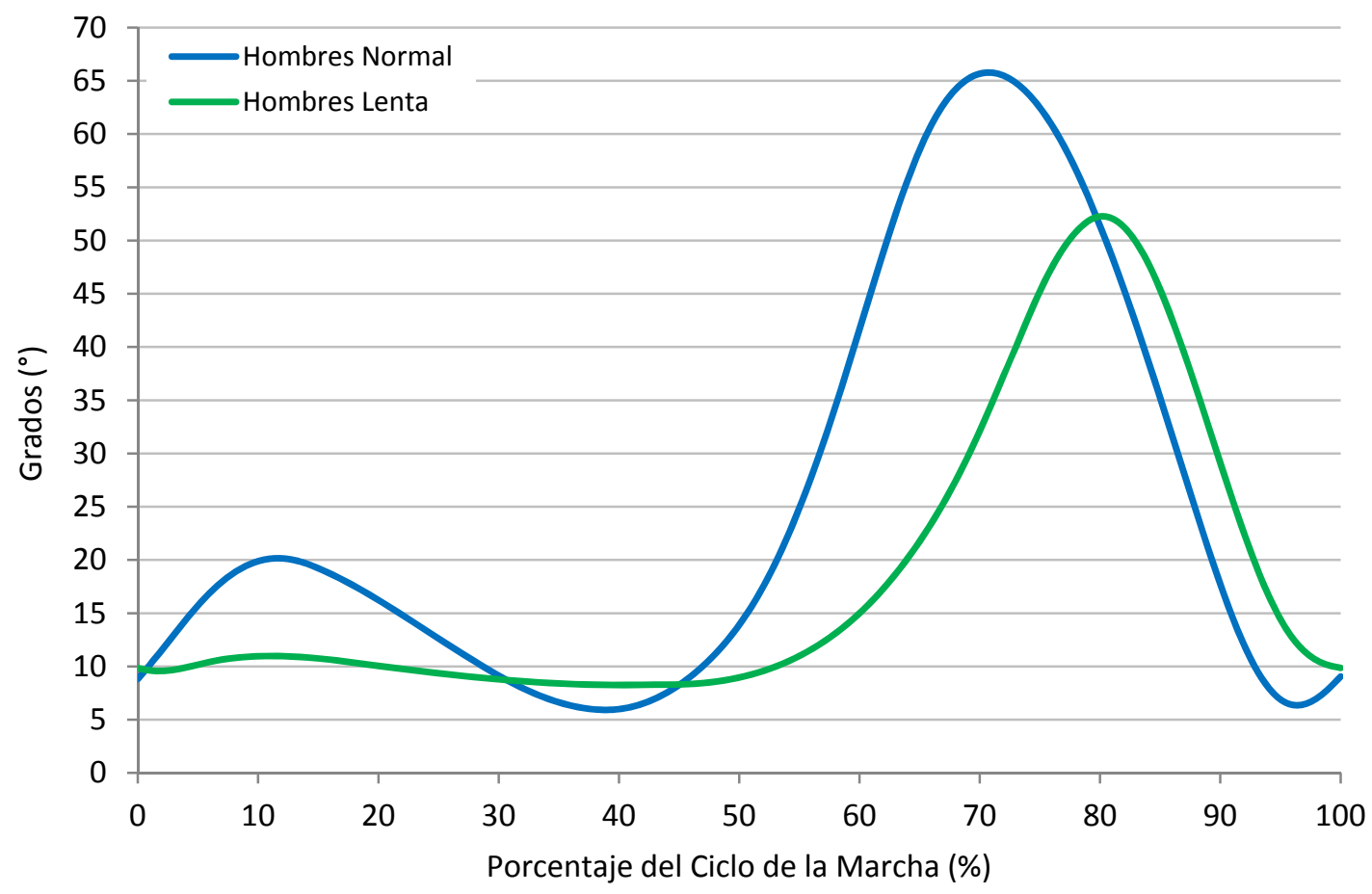

Figura 55. Cinemática de la rodilla. Diferencias entre marcha normal y marcha lenta en hombres.

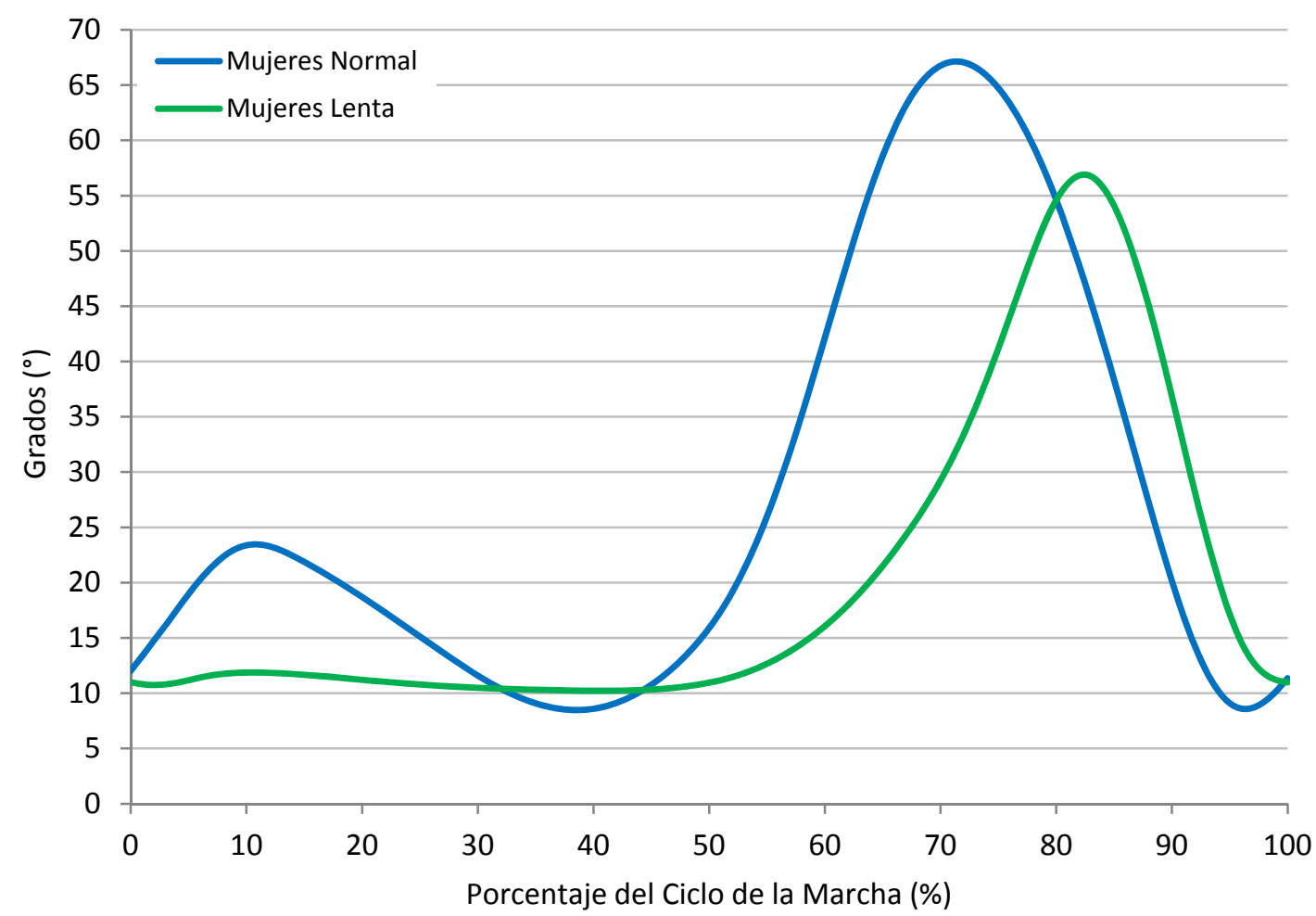

Figura 56. Cinemática de la rodilla. Diferencias entre marcha normal y marcha lenta en mujeres. 
El tobillo, como la cadera y la rodilla, presenta el máximo y mínimo de flexo-extensión del patrón lento, en hombres y mujeres, posterior a la marcha normal. El máximo, que en la marcha normal se encontraba alrededor del $45 \%$ del ciclo, se retrasa hasta el $51-52 \%$, y el mínimo, en torno al $62 \%$ del ciclo durante la marcha normal, aparece entre el $75 \%$ y el $77 \%$.

En ambos sexos el rango de movimiento disminuye fundamentalmente por la menor extensión (flexión plantar) en el instante del despegue del pie del suelo (Baker, 2013; Riley et al., 2001), $y$, aunque las diferencias entre velocidad normal y lenta en las mujeres no son significativas, hombres y mujeres presentan modificaciones similares en la cinemática del tobillo durante la marcha lenta. Riley et al. (2001), describe un aumento de la flexión (flexión dorsal) del tobillo previo al contacto del talón con el suelo como consecuencia de la disminución de la velocidad. Este aumento de la flexión dorsal concuerda con el pico de flexión, cercano al 90\% del ciclo, que no aparece en las curvas de velocidad normal (Figuras 57 y 58). Es importante señalar la posición del tobillo en el contacto inicial, ya que, independientemente de la velocidad a la que se camine, hombres y mujeres mantienen la misma flexión dorsal del tobillo. De este modo ofrecen un punto de contacto estable del miembro inferior con el suelo, que Perry (1992) caracteriza como un determinante de un patrón eficiente de la marcha que propicia la consecución de los patrones cinemáticos de cadera, rodilla y tobillo.

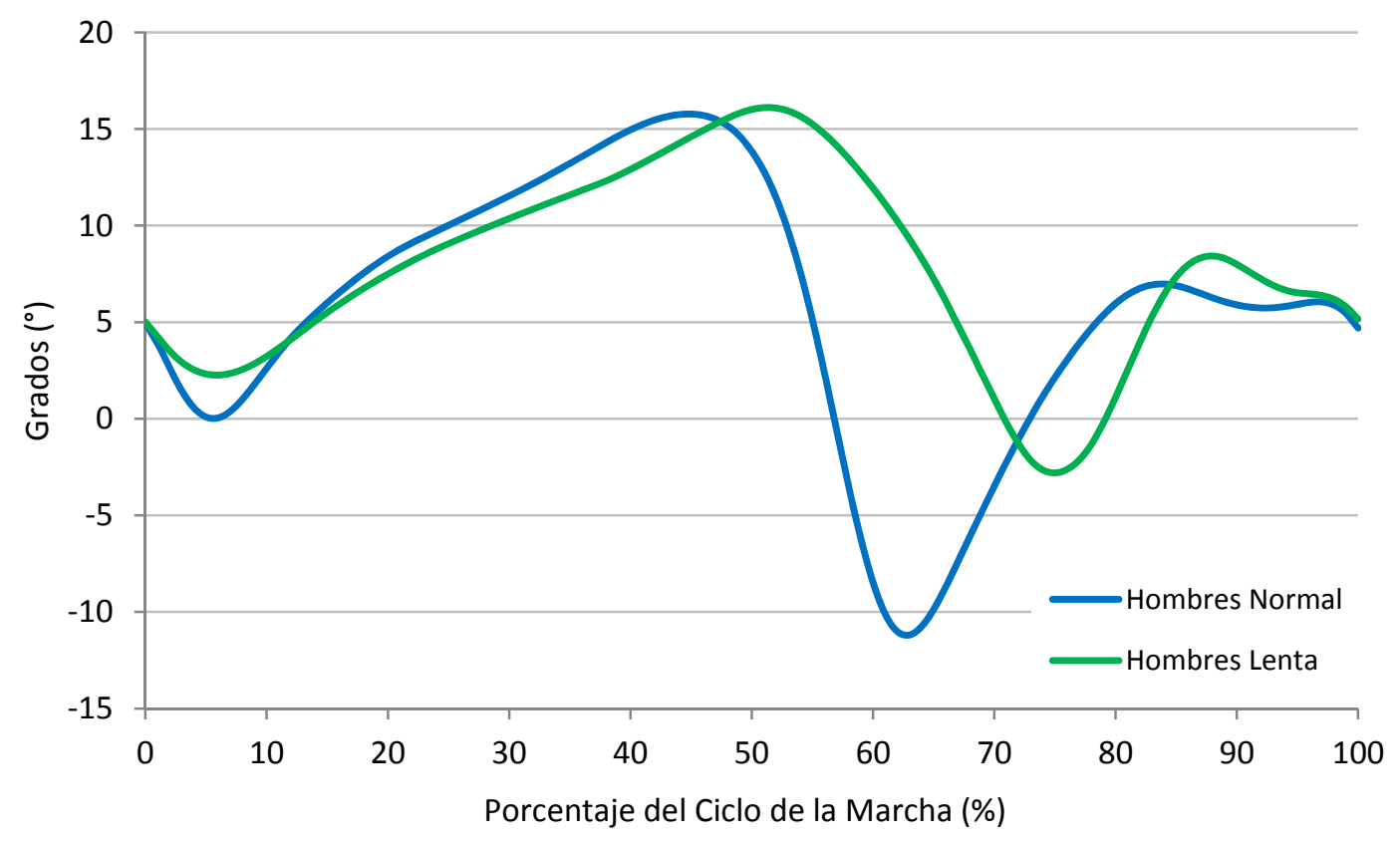

Figura 57. Cinemática del tobillo. Diferencias entre marcha normal y marcha lenta en hombres. 


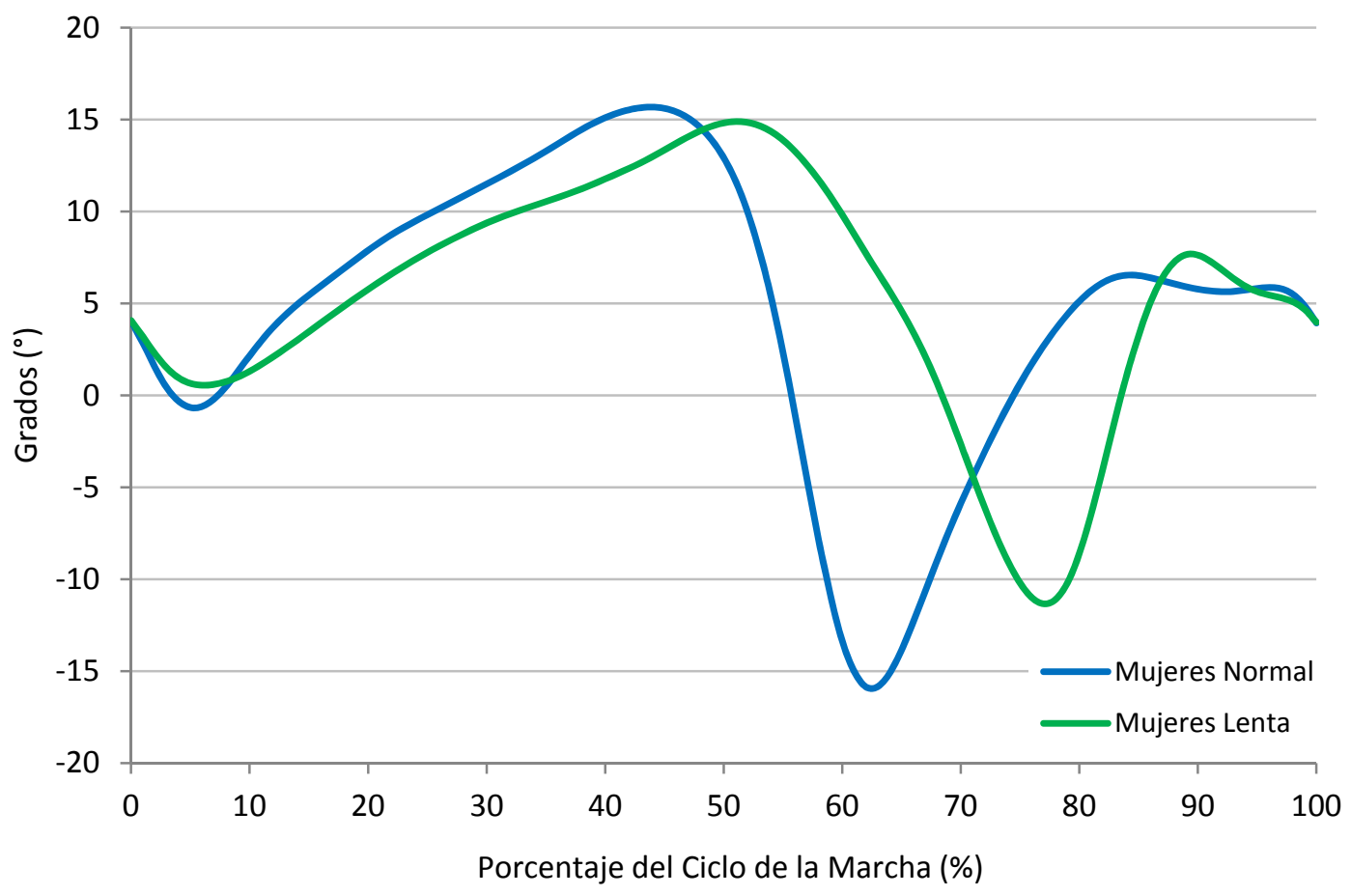

Figura 58. Cinemática del tobillo. Diferencias entre marcha normal y marcha lenta en mujeres.

La basculación lateral de la pelvis, que durante la marcha normal se señaló como una de las características más diferenciadoras entre el patrón de hombres y de mujeres, también presenta adaptaciones como consecuencia de la disminución de la velocidad. Hombres y mujeres disminuyen el movimiento de la pelvis (Bejek et al., 2006) en el plano frontal hasta alcanzar los $8-9^{\circ}$ de rango de movimiento durante la marcha lenta en ambos sexos, siendo la disminución de las mujeres proporcionalmente mayor. Esta reducción se debe a la pérdida de la necesidad de control del movimiento vertical ascendente del centro de gravedad durante las fases de apoyo monopodal por parte de la pelvis (Perry, \& Burnfield, 2010). La cadera y la rodilla, al disminuir su rango de movimiento durante la velocidad lenta, también suavizan y disminuyen el movimiento vertical (plano sagital) del centro de gravedad, que se reduce a menos de la mitad en ambos sexos (de $44 \mathrm{~mm}$ en la marcha normal a $17 \mathrm{~mm}$ en la marcha lenta) como señalan Orendurff et al. (2004) y Swinnen et al. (2013).

El aumento del movimiento lateral del centro de gravedad (plano frontal) y del ancho de paso durante la marcha a velocidad lenta supone un mayor esfuerzo para el mantenimiento del equilibrio y, por tanto, un patrón menos eficiente (Perry, 1992; Saunders et al., 1953). Aun siendo menos eficiente, todos los sujetos de ambos sexos aumentan el rango de desplazamiento lateral del centro de gravedad durante la velocidad lenta como forma de trasferencia de peso de una pierna a la otra, y aumentan el ancho del paso con el objetivo de 
ampliar la base de sustentación y evitar el desequilibrio (Orendurff et al., 2004; Swinnen et al., 2013). Hombres y mujeres mantienen, en la marcha lenta, las diferencias que se señalaban durante la marcha normal, siendo el movimiento lateral del centro de gravedad y el ancho de paso mayor en los hombres que en las mujeres con independencia de la velocidad a la que se camine.

Ambos sexos, independientemente de la estatura, presentan las mismas modificaciones y utilizan similares estrategias de adaptación para caminar a una velocidad lenta. Por tanto, si todos los sujetos modifican sus patrones de manera similar, la mayoría de las particularidades y características descritas para hombres y mujeres durante la marcha a velocidad normal cambiarían proporcionalmente y las diferencias se mantendrían estables tras la adaptación del patrón a una velocidad lenta. 


\subsection{Comparativa de los patrones de la marcha reproducidos por el Exoesqueleto con el $30 \%$,} $50 \%$ y $70 \%$ de descarga de peso corporal y los patrones de la Marcha a velocidad Lenta

Durante el análisis de la marcha lenta se ha constatado que las diferencias entre sexos, que se encontraban en el patrón de la marcha normal, se mantienen aun disminuyendo la velocidad a $25 \mathrm{~cm} / \mathrm{s}$. A continuación se estudiará el comportamiento de hombres y mujeres, y su adaptación, al patrón de marcha lenta reproducido por el exoesqueleto a diferentes porcentajes de descarga de peso $(30 \%, 50 \%$ y $70 \%)$, que, aunque resulta ser un patrón preestablecido, sufre modificaciones dependiendo de las características anatómicas y biomecánicas de cada individuo.

Analizando los resultados de hombres y mujeres recogidos mientras portaban el sistema Hybrid, se observa, que en ambos sexos, e independientemente al porcentaje de descarga, la velocidad de la marcha ha disminuido un $70 \%$ (hasta $8 \mathrm{~cm} / \mathrm{s}$ ) desde la velocidad teórica a la que el exoesqueleto reproduciría los patrones $(25 \mathrm{~cm} / \mathrm{s})$. Dicha disminución de velocidad se debe, principalmente, a un acortamiento del $60 \%$ de la longitud de paso con respecto al patrón de marcha lenta. La aparente falta de fuerza de los motores del exoesqueleto, que no consiguen contrarrestar la resistencia ofrecida por la contracción involuntaria de la musculatura de los sujetos sanos, tiene como consecuencia que la distancia alcanzada en cada paso sea menor, y por lo tanto haya una menor velocidad. Además, la cadencia también disminuye en los tres porcentajes de descarga con respecto a la velocidad lenta, siendo en torno a 10 pasos por minuto menor en hombres y mujeres (de 40 pasos $/ \mathrm{min}$ a 30 pasos $/ \mathrm{min}$ ).

Aunque la velocidad, longitud de paso y cadencia no difieren entre hombres y mujeres portando el dispositivo Hybrid, se han encontrado diferencias en la distribución temporal durante el ciclo de la marcha a diferentes porcentajes de descarga, $y$, principalmente en el comportamiento cinemático de las articulaciones del miembro inferior (cadera, rodilla y tobillo). La distribución porcentual de las fases de apoyo y oscilación durante el ciclo de la marcha no presenta diferencias estadísticas en hombres y mujeres, pero muestra una tendencia, señalada por otros autores (Finch, Barbeau, \& Arsenault, 1991; Threlkeld et al., 2003). A medida que aumenta el porcentaje de peso en descarga, la fase de oscilación aumenta (\%) y la fase de apoyo disminuye (\%), ya que la estabilidad que proporciona la descarga de peso corporal permite reducir el porcentaje de fase de apoyo (Threlkeld et al., 2003). En términos temporales (s), estos cambios en el patrón apoyo-oscilación (\%) se traducen de manera diferente en hombres y mujeres. Los hombres muestran una disminución significativa del tiempo de apoyo a medida que aumenta el porcentaje de descarga de peso, debido, fundamentalmente, a la misma disminución en el tiempo de doble apoyo, siendo en los tres porcentajes de descarga mayor que durante la marcha lenta (Finch et al., 1991; Van 
Kammen, Boonstra, Reinders-Messelink, \& den Otter, 2014) (Figura 59). Sin embargo, las mujeres modifican el tiempo de oscilación durante las tres condiciones de descarga, aumentando significativamente a medida que aumenta el porcentaje de descarga de peso, y siendo, mayores que durante el patrón de la marcha lenta (Figura 59).

\section{Hombres}

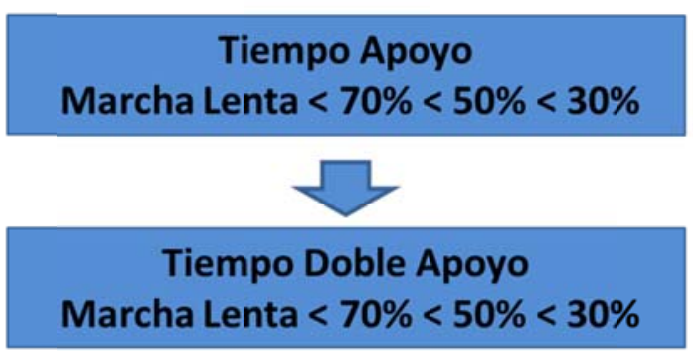

\section{Mujeres}

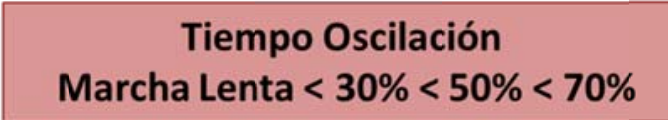

Figura 59. Diferencias en la distribución temporal entre los porcentajes de descarga y la marcha lenta (30\%, $50 \%$ y $70 \%)$ (Hombres y Mujeres)

En la cinemática global de las tres articulaciones del miembro inferior se observa como el motor de la articulación proximal tiene más fuerza y control sobre los movimientos de la cadera, mientras que la articulación más distal (el tobillo) se encuentra menos controlada por los motores del dispositivo Hybrid, y sus movimientos están más condicionados por la resistencia ofrecida por la anatomía de cada individuo y por el peso corporal soportado. La cadera, en hombres y mujeres, presenta mayor extensión durante las tres condiciones de descarga de peso que en la marcha lenta, debido a dos causas principales. La primera causa es la falta de control del motor sobre la extensión, realizada a favor de la gravedad, y que sucede mientras la pierna contralateral avanza en la fase final de oscilación (Figura 60), y, la segunda, la difícil coordinación necesaria para la transferencia de peso de una pierna a la otra, lo que hace que el motor no tenga fuerza suficiente para el comienzo de flexión de cadera si el peso corporal no se encuentra completamente liberado. Esto provoca, por tanto, que se alcancen valores mayores de extensión de cadera. Además, se puede observar que la mayor extensión de cadera, en ambos sexos, sucede con el $50 \%$ de descarga de peso, condición en la que se combina una más exigente coordinación para la transferencia de peso que en el 30\%, pero soportando todavía una gran cantidad de peso corporal. En los hombres, estas diferencias, aunque estadísticamente significativas, no tienen consecuencias sobre el comportamiento flexor de la cadera y no influyen sobre la flexión máxima de cadera o la flexiốn de cadera en el contacto inicial. Sin embargo, las mujeres muestran unas diferencias de más de $15^{\circ}$ entre la 
extensión máxima alcanzada durante la marcha lenta (menor a la de los hombres) $(10,44 \pm$ $4,60^{\circ}$ ) y la extensión máxima en el $50 \%$ de descarga de peso (mayor a la de las hombres) $(-5,69$ $\pm 1,61^{\circ}$ ). Los cambios en la extensión de cadera modifican la flexión máxima y la flexión en el contacto inicial, mayores, durante la marcha lenta que en los porcentajes de descarga de peso. Esto se debe a que, al mantener el rango de movimiento $\left(30^{\circ}\right)$ y partir de una extensión menor de cadera, se alcanzarán valores mayores de flexión máxima, y por tanto, de flexión durante el contacto inicial.

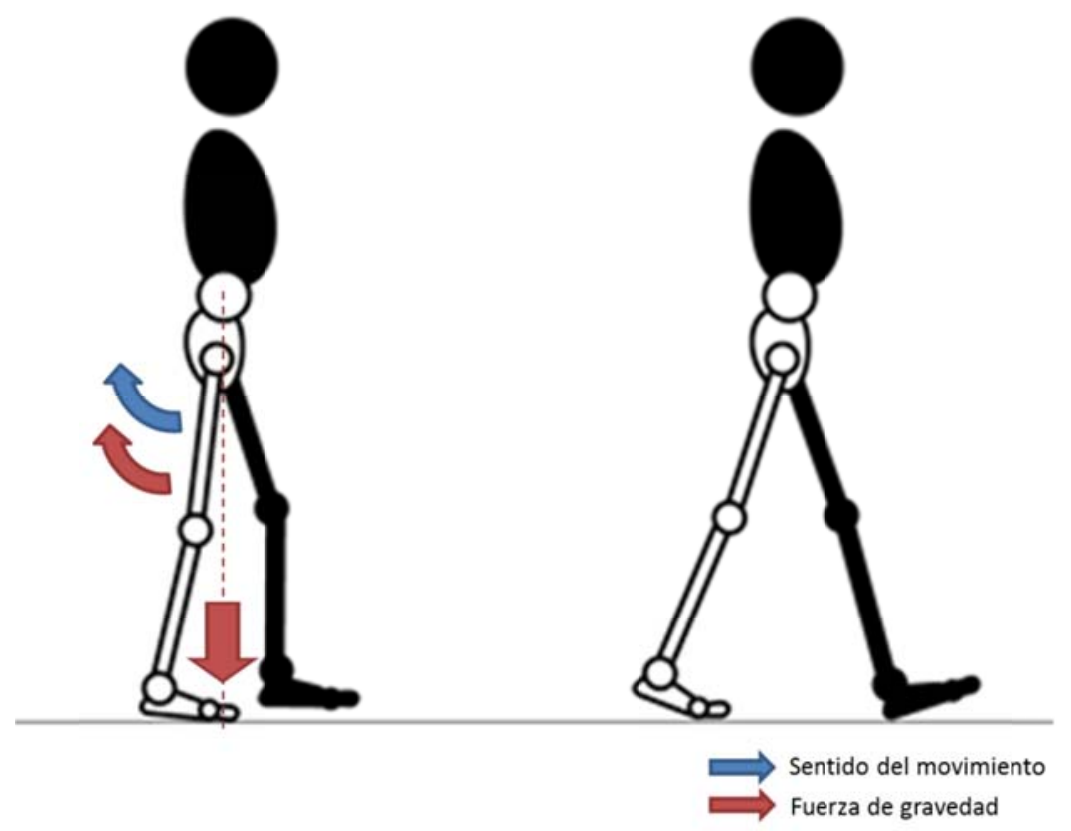

Figura 60. Cinemática de la cadera durante el instante de extensión máxima.

La extensión en la rodilla y el tobillo, al contrario que en la cadera, se realiza en contra de la fuerza de la gravedad, por lo que los motores del exoesqueleto en estas articulaciones deberían vencer el peso corporal (Figuras 61 y 62). Se observa, en ambos sexos, que la extensión del tobillo y la rodilla es menor durante las diferentes condiciones de descarga de peso que en la marcha lenta, lo que podría significar que, aun liberando el $70 \%$ del peso corporal de los sujetos, los motores no generan suficiente fuerza para extender ambas articulaciones hasta los rangos de movimiento descritos en el patrón de marcha a velocidad lenta. La falta de extensión de rodilla durante el apoyo condiciona directamente, en ambos sexos, los valores de flexión máxima y de flexión en el contacto inicial de la radilla. Al presentar menor extensión en el 30\%, 50\% y $70 \%$ de descarga de peso que en la marcha lenta, y mantener en todos los casos rangos de movimiento similares a $50^{\circ}$, es lógico pensar que se 
alcanzarán valores mayores de flexión máxima y flexión en el contacto inicial con el exoesqueleto que durante la marcha lenta.

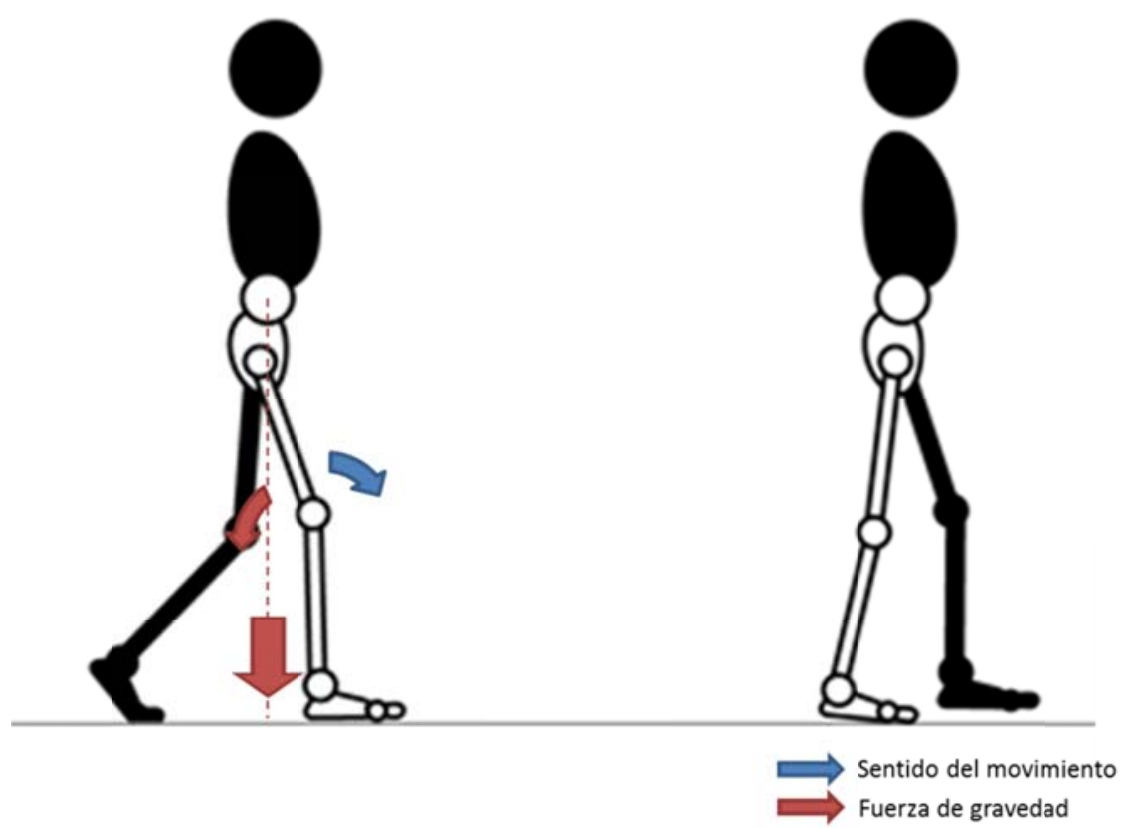

Figura 61. Cinemática de la rodilla durante el instante de extensión máxima.

Una de las diferencias entre el comportamiento cinemático del tobillo y el de la cadera y la rodilla reside en el orden en el que los máximos y mínimos de la curva ángulo articular/porcentaje del ciclo de la marcha se ordenan en el tiempo. En la cadera y la rodilla la extensión máxima precede a la flexión máxima, por lo que el comportamiento cinemático de estas articulaciones durante la extensión máxima condicionará la flexión máxima. Sin embargo, en el tobillo, la extensión máxima se encuentra posterior y no influirá en la flexión máxima. La extensión máxima o flexión plantar del tobillo, en ambos sexos, será menor durante el 30\% de descarga $\left(0,59 \pm 2,98^{\circ}\right.$ en los hombres y $0,19 \pm 5,84^{\circ}$ en las mujeres) e irá incrementando a medida que el peso descargado aumenta $\left(-6,29 \pm 5,57^{\circ}\right.$ en los hombres y $-8,78 \pm 2,48^{\circ}$ en las mujeres durante el 70\% de descarga de peso) (Threlkeld et al., 2003). Esto hace también que el rango de movimiento del tobillo aumente a medida que aumenta el porcentaje de descarga de peso. En ambos casos, extensión máxima y rango de movimiento total, los valores serán menores en las tres condiciones de descarga de peso que durante el patrón de marcha a velocidad lenta. Finch et al. (1991) y Kurz, Deffeyers, Arpin, Karst y Stuberg (2012) señalaban en sus investigaciones la disminución del rango de movimiento de las artículaciones con la descarga de peso corporal, hecho que únicamente se ha constatado en la articulación más 
distal. Esto es debido a que el movimiento articular en la presente investigación está condicionado al movimiento generado por el exoesqueleto y que, en este ca:so, la disminución de rango articular en el tobillo está ocasionada por un déficit de fuerza de los motores para vencer la resistencia del peso corporal en contra de la fuerza de la gravedad.

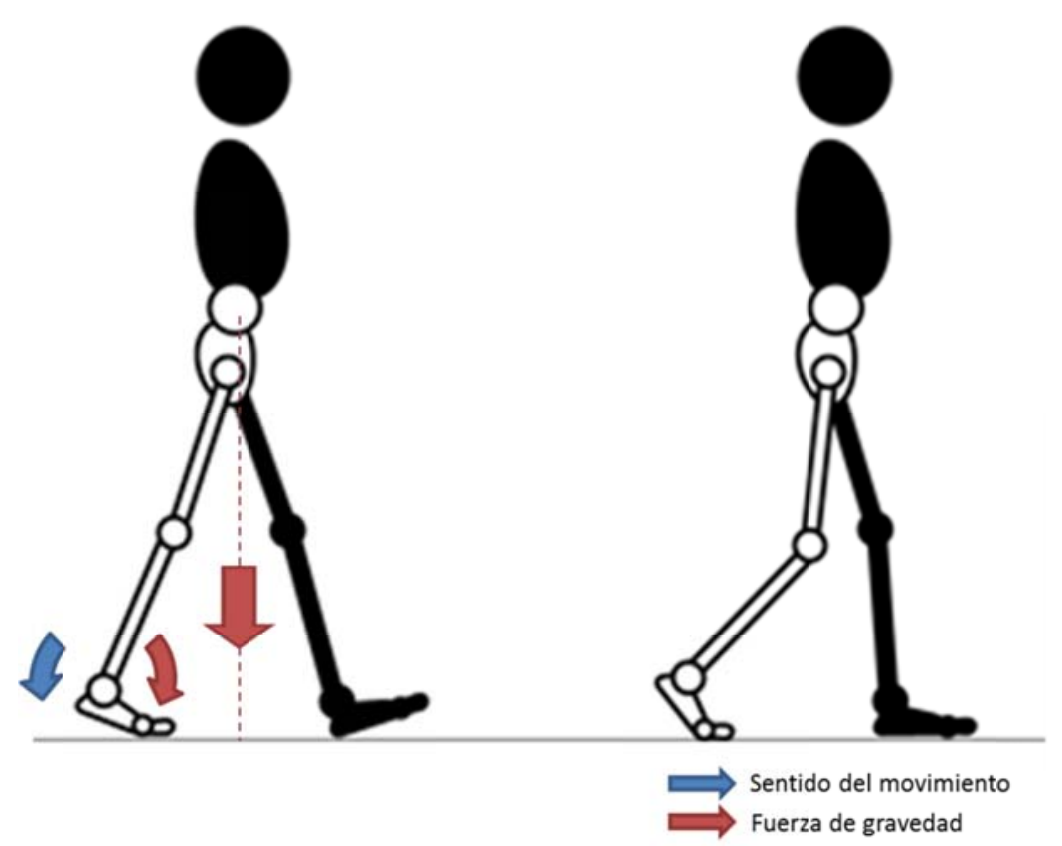

Figura 62. Cinemática del tobillo durante el instante de extensión máxima.

Durante la marcha con el dispositivo Hybrid no fue posible analizar los datos de la pelvis por la imposibilidad de adaptar el modelo de marcadores a la cesta pélvica del exoesqueleto. Además, el movimiento de la pelvis se encontró limitado durante las pruebas con el dispositivo y no se produjeron movimientos de la pelvis guiados por el exoesqueleto en el plano sagital, frontal y transversal.

Ante la falta de movimiento de la pelvis y la cadera en el plano frontal, y la limitación del desplazamiento lateral de la pelvis por la abducción y aducción de la cadera, el cambio de peso de una pierna a otra durante la marcha con el dispositivo Hybrid se realizó íntegramente por la inclinación o flexión lateral del tronco. Esta inclinación lateral de tronco en el plano frontal se ve incrementada a medida que aumenta el porcentaje de descarga de peso corporal, y está relacionada con la regulación de la transferencia de peso durante la marcha (Swinnen et al., 2015). Hombres y mujeres presentan la misma tendencia en el movimiento lateral del centro de gravedad en relación al porcentaje de descarga de peso, aunque se siguen mostrando las diferencias entre sexos que se encontraban durante la marcha normal. Los hombres, durante 
la marcha con el dispositivo Hybrid, muestran valores mayores que las mujeres en el movimiento lateral del centro de gravedad en los tres porcentajes de descarga de peso corporal. Como forma de regular el equilibrio ante el incremento del movimiento lateral del centro de gravedad, se muestra un aumento del ancho de paso a medida que aumenta el porcentaje de descarga de peso y, por tanto, de la base de sustentación. Así mismo, los hombres muestran valores mayores que las mujeres durante los tres porcentajes de descarga, manteniendo las diferencias que se observaban en la marcha normal y marcha lenta.

El aumento del centro de gravedad en el plano frontal, debido al mecanismo compensatorio de transferencia de peso realizado por el tronco, se observa junto al también incremento del movimiento vertical. El movimiento vertical durante los tres porcentajes de descarga aumenta considerablemente $(125 \mathrm{~mm})$ desde los valores observados durante la marcha lenta $(17 \mathrm{~mm})$, inferiores a los registrados en la marcha normal ( $44 \mathrm{~mm}$ ). Este incremento en el movimiento vertical en el centro de gravedad se debe a la restricción de movimiento de la pelvis con el dispositivo Hybrid, imposibilitando la basculación lateral de la pelvis, que tiene como objetivo la reducción de la amplitud del movimiento ascendente del centro de gravedad durante el apoyo monopodal (Perry, \& Burnfield, 2010).

Saunders et al. (1953) y Perry (1992) describieron, como se ha mencionado anteriormente, los determinantes que caracterizaban una marcha normal. El movimiento de la pelvis, rotación, basculación lateral y desplazamiento lateral asociado a la abducción y aducción de la cadera, se describe como uno de los mayores determinantes para considerar una marcha normal. La carencia del movimiento de la pelvis durante la marcha da como resultado una marcha en forma de "tijera" que produce compensaciones y resulta ser poco eficiente, como se ve reflejado en el aumento del movimiento vertical y lateral del centro de gravedad (Neumann, 2002; Saunders et al., 1953; Viel, \& Plas, 2002).

Por tanto, aunque existe un correcto movimiento del tobillo y posicionamiento durante el contacto inicial, una buena estabilidad del pie durante el apoyo y liberación del mismo en la fase de oscilación (Perry, 1992), no podríamos considerar la marcha reproducida por el dispositivo Hybrid apropiada para un proceso de rehabilitación de la marcha en sujetos con lesión medular incompleta con opciones de recuperar un patrón normal de la marcha. El déficit de movimiento en la pelvis en los tres planos condiciona el correcto reentrenamiento de los patrones de la marcha, al carecer del mecanismo para la transferencia de peso y amortiguación del movimiento vertical del centro de gravedad, y produce patrones poco eficientes y con alto gasto de consumo energético. Por ello, el patrón generado por el primer prototipo del dispositivo Hybrid resulta tener una cinemática básica y limitada, y no se 
DISCUSIÓN

consideraría idóneo para la rehabilitación y recuperación del patrón de la marcha. A pesar de ello, podría ser un apropiado mecanismo de compensación de la marcha en sujetos con lesión medular completa, como forma de bipedestación y locomoción para mejorar el tono muscular y la espasticidad, incrementar la eficiencia y regularidad del sistema urinario y digestivo, reducir la desmineralización ósea, mejorar el sistema cardiovascular y respiratorio, y prevenir la aparición de las escaras por presión y mejorar del estado de la piel. 
6. Conclusiones 

Se presentan a continuación las conclusiones más importantes que se extraen del presente trabajo de investigación:

En relación al objetivo 1, examinando las diferencias en la marcha normal, se acepta la hipótesis nula planteada, en la que se establece que existen diferentes patrones de la marcha a velocidad normal en función del sexo y la estatura de los sujetos:

;

$\mathcal{L}$ Las principales diferencias entre hombres y mujeres se encuentran en los parámetros cinemáticos: Mayor flexión de cadera y rodilla, y la mayor flexión plantar del tobillo en las mujeres. Aunque son la basculación lateral de la pelvis, mayor en las mujeres, y el movimiento lateral del centro de gravedad, mayor en los hombres, los parámetros diferenciadores más característicos de cada sexo.

; Las diferencias en el patrón normal en función de la estatura resultan ser menos evidentes que las diferencias entre sexos, pero deberían ser consideradas para la identificación de patrones de la marcha normales en diferentes grupos de estatura.

En relación al objetivo 2, comparando los patrones de velocidad normal y velocidad lenta, se acepta la hipótesis nula en la que se determina que el patrón de la marcha a velocidad lenta se muestra diferente al patrón a velocidad normal:

; La disminución de la velocidad de la marcha implica un acortamiento de la fase de oscilación, y, por lo tanto, un alargamiento de la fase de apoyo, lo que presenta como consecuencia el retraso de los máximos y mínimos de la curva de flexo-extensión de cadera, rodilla y tobillo en el ciclo de la marcha. Así como, una disminución de los rangos de movimiento de las tres articulaciones.

;

$\Lambda$ Ambos sexos, durante la velocidad lenta, reducen el movimiento vertical del centro de gravedad como consecuencia de la disminución del rango de movimiento de las articulaciones del miembro inferior. Por el contrario, el movimiento lateral del centro de gravedad y el ancho de paso aumentan como forma de incrementar la estabilidad y la transferencia de peso. 
;

$\Lambda$ Hombres y mujeres utilizan estrategias similares para modificar y adaptar el patrón de la marcha a una velocidad lenta $(0,25 \mathrm{~m} / \mathrm{s})$, de tal forma que se mantienen las características diferenciadoras entre hombres y mujeres que aparecían durante los patrones de la marcha a velocidad libremente seleccionada.

En relación al objetivo 3 , en la comparación del patrón a velocidad lenta y el patrón reproducido por el exoesqueleto, se acepta la hipótesis nula en la que se establece que la descarga de peso corporal con el dispositivo HYBRID y la interacción con el cuerpo del sujeto modifican el patrón de marcha lenta implementado en el dispositivo:

$\mathcal{L}$ El aumento de porcentaje de peso corporal soportado por el dispositivo HYBRID permite un mayor control de los motores en la extensión de cadera, rodilla y tobillo durante el apoyo.

$\mathcal{L}$ La falta de movimiento lateral de la pelvis con el dispositivo HYBRID condiciona la estrategia seleccionada para la transferencia de peso y aumenta el rango de movimiento de la trayectoria lateral del centro de gravedad. Por su parte, la restricción de movimiento de basculación lateral de la pelvis tiene como consecuencia el aumento del rango de movimiento de la trayectoria vertical del centro de gravedad.

Por tanto, se concluye que la interacción entre el dispositivo HYBRID con las características anatómicas y biomecánicas de los sujetos de ambos sexos, tiene como resultado un patrón de la marcha poco eficiente que no resultaría indicado para los procesos de rehabilitación o recuperación de la marcha. Por el contrario, el patrón de la marcha reproducido por el exoesqueleto podría considerarse adecuado como forma de compensación de la bipedestación y locomoción, y mejora de los procesos fisiológicos deteriorados por la sedestación mantenida. 
07. Aportaciones Finales 



\section{APLICACIONES DEL ESTUDIO}

\subsection{Aplicaciones del Estudio}

El conocimiento de las características de la marcha normal y la creación de bases de datos ofrece un estándar de referencia para los procesos de recuperación funcional o rehabilitación de la marcha. Asimismo, el establecer patrones de marcha normal diferenciados en función de algunas características individuales permite adaptar los procesos de rehabilitación, con dispositivos robóticos o con terapia manual, a cada sujeto. De esta manera cada paciente podría reaprender un patrón indicado para su edad, estatura y sexo, en vez de un patrón de la marcha genérico que no se adaptase a sus características anatómicas, biomecánicas y socioculturales.

También, en el creciente desarrollo tecnológico de dispositivos robóticos diseñados para la rehabilitación y compensación de la bipedestación y locomoción, el establecer una forma de evaluación del movimiento reproducido por los exoesqueletos y conocer su comportamiento en la interacción con el cuerpo de los sujetos ofrecería información para la mejora y adaptación de los dispositivos robóticos a cada paciente. 


\subsection{Limitaciones del Estudio}

El presente estudio ha analizado la marcha normal y la marcha lenta en sujetos sanos pertenecientes a una muestra de adultos jóvenes de la población española, lo que podría limitar la transferencia de los resultados a otros grupos de población con edades o contexto sociocultural diferente.

En cuanto a la evaluación de la marcha con el dispositivo Hybrid, como consecuencia de la poca disponibilidad del exoesqueleto y el tiempo prolongado necesario para la recogida de datos con cada sujeto se pudo analizar un número reducido de sujetos portando el dispositivo. Este hecho podría limitar el conocimiento adquirido en cuanto al funcionamiento del dispositivo, así como las adaptaciones y modificaciones en la interacción con los sujetos.

Además, el proyecto Hybrid, presentaba como objetivo ambicioso la valoración del exoesqueleto en sujetos con lesión medular. Se analizaron los criterios de inclusión de estos sujetos y se planificó su análisis en el laboratorio durante la investigación. Sin embargo, la dificultad en contactar con muestra con lesión medular que cumpliese los criterios de inclusión establecidos, así como la disponibilidad propia del dispositivo Hybrid, cuyo propietario es el Consejo Superior de Investigaciones Científicas (CSIC), imposibilitó la evaluación del sistema con dichos pacientes. 


\subsection{Futuras Líneas de Investigación}

El trabajo realizado en el presente estudio supone un punto de partida para la creación de diversas bases de datos de marcha normal y un mejor estudio en el futuro de las características de la marcha en función de múltiples variables independientes, como el sexo, la estatura, la edad, etc. Además, el conocimiento de las adaptaciones de los patrones cinemáticos y espacio-temporales a una velocidad lenta en cada uno de estos grupos de población.

Aunque, fundamentalmente, la línea de investigación que establece este estudio sería la evaluación de los diferentes dispositivos diseñados para la rehabilitación y compensación de la marcha humana en sujetos con lesión medular. Sería necesario un análisis con una muestra mayor de sujetos sanos para validar el funcionamiento y reproducción de los patrones de la marcha, y comenzar la evaluación biomecánica del dispositivo con sujetos con lesión medular para analizar la aplicabilidad de los patrones de la marcha en este tipo de pacientes. 

Bibliografía 

Adams, M.M., \& Hicks, A.L. (2011). Comparison of the effects of body-weight-supported treadmill training and tilt-table standing on spasticity in individuals with chronic spinal cord injury. The Journal of Spinal Cord Medicine, 34(5), 488-494.

Alekna, V., Tamulaitiene, M., Sinevicius, T., \& Juocevicius, A. (2008). Effect of weight-bearing activities on bone mineral density in spinal cord injured patients during the period of the first two years. Spinal Cord, 46(11), 727-732.

American Spinal Injury Association. International standards for neurological classification of spinal cord injury (ISNCSCI). Recuperado 05/08/2015, de www.asia-spinalinjury.org.

Asociación Nacional de Lesionados Medulares y Grandes Discapacitados Físicos. (2012). Análisis sobre la lesión medular en España. Toledo: Federación Nacional Aspaym.

Asociación Nacional de Lesionados Medulares y Grandes Discapacitados Físicos. (2013). Lesión Medular: Guía para el manejo del paciente con LM crónica. Madrid: ASPAYM Madrid.

Baker, R.W. (2013). Measuring walking: A handbook of clinical gait analysis. London: Mac Keith Press.

Beauchet, O., Annweiler, C., Lecordroch, Y., Allali, G., Dubost, V., Herrmann, F.R., ... Kressig, R.W. (2009). Walking speed-related changes in stride time variability: effects of decreased speed. Journal of NeuroEngineering and Rehabilitation, 6, 32-37.

Beaulieu, M.L., Lamontagne, M., \& Beaulé, P. (2010). Lower limb biomechanics during gait do not return to normal following total hip arthroplasty. Gait \& Posture, 32(2), 269-273.

Bejek, Z., Paróczai, R., Illyés, Á., \& Kiss, R.M. (2006). The influence of walking speed on gait parameters in healthy people and in patients with osteoarthritis. Knee Surgery, Sports Traumatology, Arthroscopy, 14, 612-622.

Ben, M., Harvey, L., Denis, S., Glinsky, J. Goehl, G., Chee, S., ... Herbert, R.D. (2005). Does 12 weeks of regular standing prevent loss of ankle mobility and bone mineral density in people with recent spinal cord injuries? Australian Journal of Physiotherapy, 51(4), 251-256.

Benchmarking Bipedal Locomotion. (2015). Recuperado 30/07/2015, de www.benchmarkinglocomotion.org.

Benedetti, M.G., Catani, F., Leardini, A., Pignotti, E., \& Giannini, S. (1998). Data management in gait analysis for clinical applications. Clinical Biomechanics, 13(3), 204-215.

Benedetti, M.G., Manca, M., Ferraresi, G., Boschi, M., \& Leardini, A. (2011). A new protocol for 3D assessment of foot during gait: Application on patients with equinovarus foot. Clinical Biomechanics, 26, 1033-1038.

Bohannon, R.W. (1987). Gait performance of hemiparetic stroke patients: selected variables. Archives of Physical Medicine and Rehabilitation, 68, 777-781. 
Bohannon, R.W., Andrews, A.W., \& Thomas, M.W. (1996). Walking speed: reference values and correlates for older adults. Journal of Orthopaedic \& Sports Physical Therapy, 24(2), 86-90.

Bohannon, R.W., \& Andrews, A.W. (2011). Normal walking speed: a descriptive meta-analysis. Physiotherapy, 97, 182-189.

Bovi, G., Rabuffetti, M., Mazzoleni, P., \& Ferrarin, M. (2001). A multiple-task gait analysis approach: kinematic, kinetic and EMG reference data for healthy young and adult subjects. Gait \& Posture, 33(1), 6-13.

Bow, J.C. (2014). El exoesqueleto español busca la rehabilitación de los parapléjicos. EL PAís. Recuperado 21/08/2015, de http://sociedad.elpais.com/sociedad/2014/07/07/actualidad/1404752626_048763.ht $\mathrm{ml}$

Bruening, D.A., Frimenko, R.E., Goodyear, C.D., Bowden, D.R., \& Fullenkamp, A.M. (2015). Sex differences in whole body gait kinematics at preferred speeds. Gait \& Posture, 41, 540545.

Carrascosa, A., Fernández, J.M., Fernández, C., Ferrández, A., López-Siguero, J.P., Sánchez, E., ... Yeste, Y.D. (2008). Estudios españoles de crecimiento 2008. Nuevos patrones antropométricos. Endocrinologia y Nutrición, 55(10), 484-506.

Chambers, H.G., \& Sutherland, D.H. (2002). A practical guide to gait analysis. Journal of the American Academy of Orthopaedic Surgeons, 10, 222-231.

Chen, G., Chan, C.K., Guo, Z., \& Yu, H. (2013). A review on lower extremity assistive robotic exoskeleton in rehabilitation therapy. Critical Reviews in Biomedical Engineering, 41(45), 343-363.

Chiu, M.C., \& Wang, M.J. (2007). The effect of gait speed and gender on perceived exertion, muscle activity, joint motion of lower extremity, ground reaction force and heart rate during normal walking. Gait \& Posture, 25, 385-392.

Chiu, M-C., Wu, H-C., \& Chang, L-Y. (2013). Gait speed and gender effects on center of pressure progression during normal walking. Gait \& Posture, 37, 43-48.

Cho, S.H., Park, J.M., \& Kwon, O.Y. (2004). Gender differences in three dimensional gait analysis data from 98 healthy Korean adults. Clinical Biomechanics, 19, 145-152.

Chung, M-J., \& Wang, M-J.J. (2010). The change of gait parameters during walking at different percentage of preferred walking speed for healthy adults aged 20-60 years. Gait \& Posture, 31, 131-135.

Colombo, G., Joerg, M., Schreier, R., \& Dietz, V. (2000). Treadmill training of paraplegic patients with a robotic orthosis. Journal of Rehabilitation Research \& Development, 37(6), 693700. 
Crosbie, J., Vachalathiti, R., \& Smith, R. (1997). Age, gender and speed effects on spinal kinematics during walking. Gait \& Posture, 5, 13-20.

Davis, R.B., Ounpuu, S., Tyburski, D., \& Gage, J.R. (1991). A gait analysis data collection and reduction technique. Human Movement Science, 10, 575-587.

Del-Ama, A.J., Koutsou, A.D., Moreno, J.C., de-los-Reyes, A., Gil-Agudo, A., \& Pons, J.L. (2012). Review of hybrid exoskeletons to restore gait following spinal cord injury. Journal of Rehabilitation Research \& Development, 49(4), 497-514.

Della Croce, U., Riley, P.O., Lelas, J.L., \& Kerrigan, D.C. (2001). A refined view of the determinants of gait. Gait \& Posture, 14, 79-84.

Den Otter, A.R., Geurts, A.C.H., Mulder, T., \& Duysens, J. (2004). Speed related changes in muscle activity from normal to very slow walking speeds. Gait \& Posture, 19, 270-278.

Dionyssiotis, Y., Lyritis, G.P., Mavrogenis, A.F., \& Papagelopoulos, P.J. (2011). Factors influencing bone loss in paraplegia. Hippokratia, 15(1), 54-59.

Ditunno, P.L., Patrick, M., Stineman, M., \& Ditunno, J.F. (2008). Who wants to walk? Preferences for recovery after $\mathrm{SCl}$ : a longitudinal and cross-sectional study. Spinal Cord, 46, 500-506.

Ditunno, J., \& Scivoletto, G. (2009). Clinical relevance of research applied to clinical trials in spinal cord injury. Brain Research Bulletin, 78, 35-42.

Dollar, A.M., \& Herr, H. (2008). Lower extremity exoskeletons and active orthoses: Challenges and state-of-the-art. IEEE Transactions on Robotics, 24(1), 144-158.

Donati, M., Vitiello, N., De Rossi, S.M.M., Lenzi, T., Crea, S., Persichetti, A., ... Carrozza, M.C. (2013). A flexible sensor technology for the distributed measurement of interaction pressure. Sensors, 13, 1021-1045.

Ekso Bionics. (2015). Ekso. Recuperado 30/07/2015, de www.eksobionics.com.

Eng, J.J., Levins, S.M., Townson, A.F., Mah-Jones, D., Bremner, J., \& Huston, G. (2001). Use of prolonged standing for individuals with spinal cord injuries. Journal of the American Physical Therapy Association, 81, 1392-1399.

Esclarín-De Ruz, A., Alcobendas-Maestro, M., Casado-López, R., Muñoz-González, A., FloridoSánchez, M.A., \& González-Valdizán, E. (2009). Sistema robotizado para la reeducación de la marcha en pacientes con lesión medular incompleta. Revista de Neurología, 49(12), 617-622.

Finch, L., Barbeau, H., \& Arsenault, B. (1991). Influence of body weight support on normal human gait: development of a gait retraining strategy. Physical Therapy, 7, 842-855.

Fukuchi, R.K., Arakaki, C., Veras Orselli, M.I., \& Duarte, M. (2010). Evaluation of alternative technical markers for the pelvic coordinate system. Journal of Biomechanics, 43(3), 592-594 
Frimenko, R., Goodyear, C., \& Bruening, D. (2015). Interactions of sex and aging on spatiotemporal metrics in non-pathological gait: a descriptive meta-analysis. Journal of Physiotherapy, 101(3), 266-272.

Fritz, S.L., Merlo-Rains, A.M., Rivers, E.D., Peters, D.M., Goodman, A., Watson, E.T., ... McClenaghan, B.A. (2011). An intensive intervention for improving gait, balance, and mobility in individuals with chronic incomplete spinal cord injury: a pilot study of activity tolerance and benefits. Archives of Physical Medicine and Rehabilitation, 92, 1776-1784.

Gard, S.A., \& Childress, D.S. (1999). The influence of stance-phase knee flexion on the vertical displacement of the trunk during normal walking. Archives of Physical Medicine and Rehabilitation, 80, 26-32.

Gil-Agudo, A., Pérez-Nombela, S., Forner-Cordero, A., Pérez-Rizo, E., Crespo-Ruiz, B., \& del Ama-Espinosa, A. (2011). Gait kinematic analysis in patients with a mild form of central cord síndrome. Journal of Neuroengineering and Rehabilitation, 8, 7.

Gomez, M., López de Subijana, C., Urendes, E., \& Navarro, E. (2015). Validation of a marker model for gait analysis with wearable exoskeletons. Journal of Mechanics Engineering and Automation, 5(7), 407-410.

Hallemans, A., Ortibus, E., Meire, F., \& Aerts, P. (2010). Low vision affects dynamic stability of gait. Gait \& Posture, 32(4), 547-551.

Harvey, L.A., Lin, C-W.C., Glinsky, J.V., \& De Wolf, A. (2009). The effectiveness of physical interventions for people with spinal cord injuries: a systematic review. Spinal Cord, 47, 184-195.

Herr, H. (2009). Exoskeletons and othoses: classification, design challenges and future directions. Journal of NeuroEngineering and Rehabilitation, 6, 21-29.

Hirasaki, E., Moore, S.T., Raphan, T., \& Cohen, B. (1999). Effects of walking velocity on vertical head and body movements during locomotion. Experimental Brain Research, 127, 117130.

Hof, L. (1996). Scaling data to body size. Gait \& Posture, 4, 222-223.

Jordan, K., Challis, J.H., \& Newell, K.M. (2007). Walking speed influences on gait cycle variability. Gait \& Posture, 26, 128-134.

Kang, H.G., \& Dingwell, J.B. (2007). Separating the effects of age and walking speed on gait variability. Gait \& Posture, 27(4), 572-577.

Kavanagh, J.J. (2009). Lower trunk motion and speed-dependence during walking. Journal of NeuroEngineering and Rehabilitation, 6, 9-18.

Kerrigan, D.C., Della Croce, U., Marciello, M., \& Riley, P.O. (2000). A refined view of the determinants of gait: significance of heel rise. Archives of Physical Medicine and Rehabilitation, 81, 1077-1080. 
Kerrigan, D.C., Riley, P.O., Lelas, J.L., \& Della Croce, U. (2001). Quantification of pelvic rotation as a determinant of gait. Archives of Physical Medicine and Rehabilitation, 82, 217-220.

Kerrigan, D.C., Todd, M.K., \& Della Croce, U. (1998). Gender differences in joint biomechanics during walking: normative study in young adults. American Journal of Physical Medicine \& Rehabilitation, 77(1), 2-7.

Kirtley, C. (2006). Clinical gait analysis: Theory and practice. Londres: Elsevier Churchill Livingstone.

Krawetz, P., \& Nance, P. (1996). Gait analysis of spinal cord injured subjects: effects of injury level and spasticity. Archives of Physical Medicine and Rehabilitation, 77, 635-638.

Kuo, A.D. (2007). The six determinants of gait and the inverted pendulum analogy: A dynamic walking perspective. Human Movement Science, 26, 617-656.

Kurz, M.J., Deffeyers, J.E., Arpin, D.J., Karst, G.M., \& Stuberg, W.A. (2012). Influence of lower body pressure support on the walking patterns of healthy children and adults. Journal of Applied Biomechanics, 28, 530-541.

Levi, R., \& Hultling, C. (1999). Spinalis Handbook: A new front for injured backs. Estocolmo: Spinalis.

Linley, H.S., Sled, E.A., Culham, E.G., \& Deluzio, K.J. (2010). A biomechanical analysis of trunk and pelvis motion during gait in subjects with knee osteoarthritis compared to control subjects. Clinical Biomechanics, 25(10), 1003-1010.

Low, K.H. (2011). Robot-assisted gait rehabilitation: From exoskeletons to gait systems. En 2011 Defense Science Research Conference and Expo (DRS), 1-10.

Luu, T.P., Low, K.H., Qu, X., Lim, H.B., \& Hoon, K.H. (2014). Hardware development and locomotion control strategy for an over-ground gait trainer: NaTUre-gaits. IEEE Journal of Translational Engineering in Health and Medicine, 2, 1-9.

Marsi Bionics. (2015). Atlas 2020. Recuperado 30/07/2015, de www.marsibionicses.weebly.com.

Mather, G., \& Murdoch, L. (1994). Gender discriminations in biological motion displays base on dynamic cues. Proceedings of the Royal Society of London B, 258, 273-279.

Medical Expo. (2015). Lokomat \& ReoAmbulator. Recuperado 30/07/2015, de www.medicalexpo.es.

Melis, E.H., Torres-Moreno, R., Barbeau, H., \& Lemaire, E. (1999). Analysis of assisted-gait characteristics in persons with incomplete spinal cord injury. Spinal Cord, 37, 430-439.

Motion Capture Studio. (2009). Motion Capture Tutorial. Recuperado 15/08/2015, de https://www.evl.uic.edu/sjames/mocap/. 
Murray, M.P., Drought, A.B., \& Kory, R.C. (1964). Walking patterns of normal men. Journal of Bone and Joint Surgery American, 46(A), 335-360.

Murray, M.P., Kory, R.C., \& Sepic, S.B. (1970). Walking patterns of normal women. Archives of Physical Medicine and Rehabilitation, 51, 637-650.

Muybridge, E. (1955). The human figure in motion. Mineola, NY: Dover Publications, Inc.

Neumann, D.A. (2002). Kinesiology of the musculoskeletal system: Foundations for physical rehabilitation. St Louis: Mosby.

Nigg, B.M., Fisher, V., \& Ronsky, J.L. (1994). Gait characteristics as a function of age and gender. Gait \& Posture, 2, 213-220.

Nymark, J.R., Balmer, S.J., Melis, E.H., Lemaire, E.D., \& Millar, S. (2005). Electromyographic and kinematic nondisabled gait differences at extremely slow overground and treadmill walking speeds. Journal of Rehabilitation Research \& Development, 42(4), 523-534.

Olney, S., \& Richards, C. (1996). Hemiparetic gait following stroke. Part I: Characteristics. Gait \& Posture, 4, 136-148.

Orendurff, M.S., Segal, A.D., Klute, G.K., Berge, J.S., Rohr, E.S., \& Kadel, N.J. (2004). The effect of walking speed on center of mass displacement. Journal of Rehabilitation Research \& Development, 41(6A), 829-834.

Pépin, A., Norman, K.E., \& Barbeau, H. (2003a). Treadmill walking in incomplete spinal-cordinjured subjects: 1 . Adaptation to changes in speed. Spinal Cord, 41, 257-270.

Pépin, A., Ladouceur, M., \& Barbeau, H. (2003b). Treadmill walking in incomplete spinal-cordinjured subjects: 2 . Factors limiting the maximal speed. Spinal Cord, 41, 271-279.

Perry, J. (1992). Gait Analysis Normal and Pathological Function. Thorofare, NJ: SLACK Inc.

Perry, J., \& Burnfield, J.M. (2010). Gait Analysis Normal and Pathological Function (2ª edición). Thorofare, NJ: SLACK Inc.

Pierrynowski, M.R., \& Galea, V. (2001). Enhancing the ability of gait analyses to differentiate between groups: scaling data to body size. Gait \& Posture, 13, 193-201.

Plas, F., Viel, E., \& Blanc, Y. (1984). La marcha humana: cinesiología dinámica, biomecánica y patomecánica. Paris: MASSON, S.A.

Prat Pastor, J.M. (1999). Biomecánica de la marcha humana patológica. En J.M. Prat Pastor (Coord.), Biomecánica de la marcha humana normal y patológica (pp. 125-201). Valencia: Instituto de Biomecánica de Valencia.

ReWalk. (2015). ReWalk Personal 6.0 System. Recuperado 30/07/2015, de www.rewalk.com.

Rex Bionics. (2015). Rex. Recuperado 30/07/2015, de www.rexbionics.com. 
Riley, P.O., Della Croce, U., \& Kerrigan, D.C. (2001). Propulsive adaptation to changing gait speed. Journal of Biomechanics, 34, 197-202.

Robotics and Rehabilitation Laboratory (ROAR Lab). (2015). ALEX. Recuperado 30/07/2015, de www.roar.me.columbia.edu/projects/alex/.

Røislien, J., Skare, Ø., Gustavsen, M., Broch, N.L., Rennie, L., \& Orpheim, A. (2009). Simultaneous estimation of effects of gender, age and walking speed on kinematic gait data. Gait \& Posture, 30, 441-445.

Sagawa, Y. Jr., Turcot, K., Armand, S., Thevenon, A., Vuillerme, N., \& Watelain, E. (2011). Biomechanics and physiological parameters during gait in lower-limb amputees: a systematic review. Gait \& Posture, 33(4), 511-526.

Sánchez Lacuesta, J.J. (1999). Biomecánica de la marcha humana normal. En J.M. Prat Pastor (Coord.), Biomecánica de la marcha humana normal y patológica (pp. 31-121). Valencia: Instituto de Biomecánica de Valencia.

Saunders, M., Inman, V.T., \& Eberhart, H.D. (1953). The major determinants in normal and pathological gait. Journal of Bone and Joint Surgery American, 35, 543-558.

Schwarts, M.H., Rozumalski, A., \& Trost, J.P. (2008). The effect of walking speed on the gait of typically developing children. Journal of Biomechanics, 41(8), 1639-1650.

Shemmell, J., Johansson, J., Portra, V., Gottlieb, G.L., Thomas, J.S., \& Corcos, D.M. (2007). Control of interjoint coordination during the swing phase of normal gait at different speeds. Journal of NeuroEngineering and Rehabilitation, 4, 10-23.

Shields, R.K., \& Dudley-Javoroski, S. (2005). Monitoring standing wheelchair use after spinal cord injury: A case report. Disability and Rehabilitation, 27(3), 142-146.

Sliwinski, M.M., Sisto, S.A., Batavia, M., Chen, B., \& Forrest, G.F. (2001). Dynamic stability during walking following unilateral total hip arthroplasty. Gait \& Posture, 19(2), 141147.

Smith, L.K., Lelas, J.L., \& Kerrigan, D.C. (2002). Gender differences in pelvic motions and center of mass displacement during walking: stereotypes quantified. Journal of Women's Health \& Gender-Based Medicine, 11, 453-458.

Studyblue. (2015). Recuperado 24/07/2015, de www.studyblue.es.

Swinnen, E., Baeyens, J-P., Pintens, S., Buyl, R., Goossens, M., Meeusen, R., ... Kerckhofs, E. (2013). Walking more slowly than with normal velocity: The influence on trunk and pelvis kinematics in young and older healthy persons. Clinical Biomechanics, 28, 800806.

Swinnen, E., Baeyens, J-P., Hens, G., Knaepen, K., Beckwée, D., Michielsen, M., ... Kerckhofs, E. (2015). Body weight support during robot-assisted walking: Influence on the trunk and pelvis kinematics. NeuroRehabilitation, 36(1), 81-91. 
Swinnen, E., Duerinck, S., Baeyens, J-P., Meeusen, R., \& Kerckhofs, E. (2010). Effectiveness of robot-assisted gait training in persons with spinal cord injury: a systematic review. Journal of Rehabilitation Medicine, 42, 520-526.

Swortec. (2015). WalkTrainer. Recuperado 01/08/2015, de www.swortec.ch/index.php/products/walktrainer.

Tanawongsuwan, R., \& Bobick, A. (2003). Performance analysis of time-distance gait parameters under different speeds. En $4^{\text {th }}$ International Conference AVBPA 2003. Guildford, UK: 2003 Proceedings.

Technaid. (2015). H2 Exoesqueleto. Recuperado 30/07/2015, de www.technaid.com.

Tesio, L., Lanzi, D., \& Detrembleur, C. (1998). The 3-D motion of the centre of gravity of the human body during level walking. I. Normal subjects at low and intermediate walking speeds. Clinical Biomechanics, 13(2), 77-82.

Theologis, T. (2009). Gait and clinical movement analysis research priorities: 2007 update from the research committee of the gait and clinical movement analysis society. Gait \& Posture, 29, 169-191.

Thomas, E.E., De Vito, G., \& Macaluso, A. (2007). Physiological cost and temporo-spatial parameters of walking on a treadmill vary with body weight unloading and speed in both healthy young and older women. European Journal of Applied Physiology, 100, 293-299.

Threlkeld, A.J., Cooper, L.D., Monger, B.P., Craven, A.N., \& Haupt, H.G. (2003). Temporospatial and kinematic gait alterations during treadmill walking with body weight suspension. Gait \& Posture, 17(3), 235-245.

Torricelli, D., González-Vargas, J., Veneman, J.F., Mombaur, K., Tsagarakis, N., del-Ama, A.J., ... Pons, J.L. (2015). Benchmarking bipedal locomotion. A unified scheme for humanoids, wearable robots, and humans. IEEE Robotics \& Automation Magazine, 22(3), 103-115.

Troje, N.F. (2002). Decomposing biological motion: a framework for analysis and synthesis of human gait patterns. Journal of Vision, 2, 371-387.

Urendes, E., Asín, G., Gómez, M., Ceres, R., \& Pons J.L. (2014). An integrated lower limb exoskeleton and body weight support system: integration and usability. En International Workshop of Wearable Robotics 2014 (WeRob). Baiona, Spain.

Van Kammen, K., Boonstra, A., Reinders-Messelink, H., \& den Otter, R. (2014). The combined effects of body weight support and gait speed on gait related muscle activity: A comparison between walking in the Lokomat exoskeleton and regular treadmill walking. PLOS ONE, 9(9), e107323.

Vicon. (2003). Vicon user manual. Oxford Metrics, Oxford. 
Viel, E., \& Plas, F. (2002). Movimientos del esqueleto: del inicio de la marcha a la velocidad de crucero. En E. Viel (Ed.), La marcha humana, la carrera y el salto. Biomecánica, exploraciones, normas y alteraciones (pp. 1-26). Barcelona: MASSON, S.A.

Waters, R.L., Yakura, J.S., Adkins, R., \& Barnes, G. (1989). Determinants of gait performance following spinal cord injury. Archives of Physical Medicine and Rehabilitation, 70, 811818.

Watt, J.R., Franz, J.R., Jackson, K., Dicharry, J., Riley, P.O., \& Kerrigan, D.C. (2010). A threedimensional kinematic and kinetic comparison of overground and treadmill walking in healthy elderly subjects. Clinical Biomechanics, 25(5), 444-449.

Weiss, R.J., Wretenberg, P., Stark, A., Palmblad, K., Larsson, P., Gröndal, L., ... Broström, E. (2008). Gait patterns in rheumatoid arthritis. Gait \& Posture, 28(2), 229-234.

Whittle, M.W. (2007). Gait analysis: an introduction (4ㅁ edición). Filadelfia: Butterworth Heinemann (Elsevier).

Widerström-Noga, E.G., Felipe-Cuervo, E., Broton, J.G., Duncan, R.C., \& Yezierski, R.P. (1999). Perceived difficulty in dealing with consequences of spinal cord injury. Archives of Physical Medicine and Rehabilitation, 80, 580-586.

Wirz, M., Bastiaenen, C., de Bie, R., \& Dietz, V. (2011). Effectiveness of automated locomotor training in patients with acute incomplete spinal injury: A randomized controlled multicenter trial. BMC Neurology, 11, 60-64.

Woltring, H.J. A Fortran package for generalized, cross-validation spline smoothing and differentiation. Advances in Engineering Software, 8(2), 104-113.

World Health Organitation. (2013). International perspectives on spinal cord injury. WHO Library Cataloguing-in-Publication Data.

Yoon, J., Park, H-S., \& Damiano, D.L. (2012). A novel walking speed estimation scheme and its application to treadmill control for gait rehabilitation. Journal of NeuroEngineering and Rehabilitation, 9, 62-74.

ZFlo Motion Analysis Systems. (2015). Reflective Markers. Recuperado 04/08/2015, de www.zflomotion.com. 

Anexos 

Anexo I 


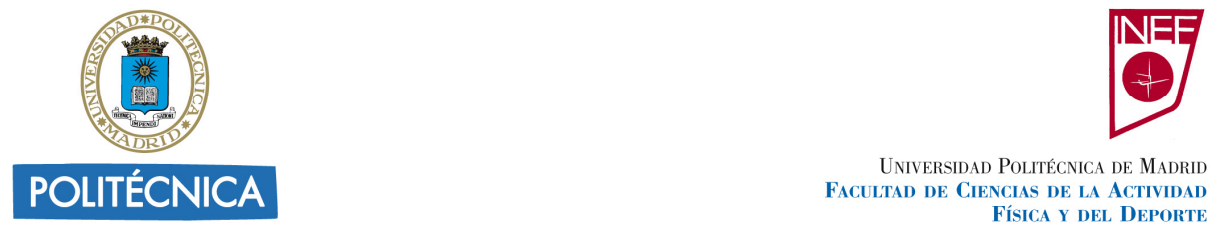

\section{CONSENTIMIENTO INFORMADO PROYECTO HYBRID}

El proyecto Hybrid tiene como principal objetivo el desarrollo de un sistema de ayuda a la marcha en Lesionados Medulares, dicho proyecto pertenece a la convocatoria fundamental no orientada I+D+i del Ministerio de Economía y Competitividad, y tiene un numero de registro DPI201128160-C03-02. El director de dicho proyecto es Enrique Navarro Cabello.

De acuerdo con las directrices éticas dictadas en la Declaración de Helsinki enmendadas en la 59a Asamblea General de la AMM en Seúl, octubre 2008, la Ley 14/2007, de 3 de julio, de Investigación Biomédica, Convenio de Oviedo relativo a los Derechos Humanos y la Biomedicina (1997), se detallan los posibles riesgos que conlleva la participación en este proyecto:

1) Daños producidos por una posible caída

2) Molestias debido al arnés que sujeta al individuo

3) Teniendo en cuenta que el exoesqueleto es un sistema electromecánico, aunque es muy improbable, se podría producir alguna molestia por una posible descarga eléctrica de muy bajo voltaje.

yo $D / D^{a}$ siendo conocedor de los riesgos e implicaciones que conlleva la participación en el presente estudio, autoriza la realización de las pruebas detalladas sobre mi persona, por el Laboratorio de Biomecánica Deportiva de la Facultad de Ciencias de la Act. Física y el Deporte-INEF, eximiendo de toda responsabilidad y renunciando a ejercer acciones legales contra dicha entidad o personal del mismo.

Estos datos están sujetos al tratamiento automatizado a que se refiere la Ley Orgánica 15/1999, de 13 de diciembre, sobre la Protección de Datos de Carácter Personal, el R. D. 1720/2007, de 21 de diciembre, por el que se aprueba el Reglamento de desarrollo de la dicha Ley, siendo los resultados de las mismas confidenciales, y permito que se traten los datos con la única finalidad científica, en tanto se respeten la confidencialidad y el anonimato de los datos, y la cesión y el tratamiento se efectúe previo procedimiento de disociación, de forma que no puedan ser expuestos en relación con mi persona.

En Madrid a de. de 2013

Firma Interesado:

Firma Responsable del Proyecto:

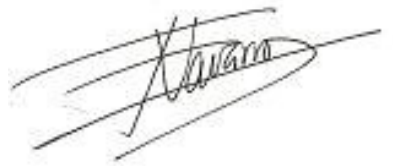

Fdo.: Dr. Enrique Navarro Cabello

Para ejercer su derecho a información, rectificación y anulación de sus datos, deberá ponerse en contacto con el Laboratorio de Biomecánica Deportiva de la Facultad de Ciencias de la Act. Física y el Deporte-INEF, a través del teléfono 913364121 o vía email al laboratorio.biomecanica.inef@upm.es 

Anexo II 



\section{INFORME QUE EMITE EL COMITÉ DE ÉTICA DE LA UNIVERSIDAD POLITÉCNICA DE MADRID}

A petición del Vicerrector de Investigación de la Universidad Politécnica de Madrid, D. Gonzalo León Serrano, de un dictamen sobre los aspectos éticos del

Proyecto "Validación de la plataforma HYBRID para la modelización y el entrenamiento de la marcha" cuyo investigador principal es Enrique Navarro Cabello del Departamento de Salud y Rendimiento Humano de la Facultad de Ciencias de la Actividad Física y del Deporte (INEF), tras los pertinentes análisis, estudios y consultas, la Comisión emite el siguiente informe:

Dado que por un lado el responsable del proyecto asegura conocer los procesos y protocolos relativos a la protección de datos y experimentación con humanos, y dado que todos los sujetos serán previamente informados sobre los objetivos del proyecto firmando un consentimiento informado para su participación en el estudio, tal y como se muestra en los Formularios adjuntos,

No hay inconveniente en informar favorablemente la propuesta, recomendando el efectivo cumplimiento de los compromisos declarados.

Y para que así conste, firmo el presente informe en Madrid a veinticinco de octubre de dos mil doce.
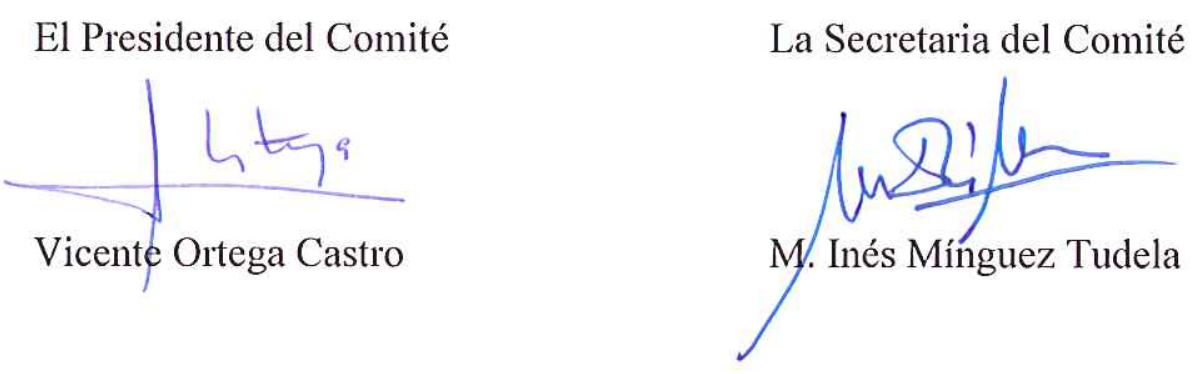

Anexo III 

Fecha:

COD.

\begin{tabular}{|l|l|}
\hline Nombre y Apellidos \\
\hline Peso & Fecha de nacimiento \\
\hline Altura & Diagnóstico \\
\hline Inter-asis distance & \\
\hline
\end{tabular}

\begin{tabular}{|c|c|c|}
\cline { 2 - 3 } \multicolumn{1}{c|}{ IZQ } & DCHA \\
\hline Asis trocánter distance $(\mathrm{cm})$ & & \\
\hline Longitud pierna $(\mathrm{cm})$ & & \\
\hline Anchura rodilla $(\mathrm{cm})$ & & \\
\hline Anchura tobillo $(\mathrm{cm})$ & & \\
\hline Longitud Pie $(\mathrm{cm})$ & & \\
\hline \multirow{2}{*}{$\begin{array}{c}\text { Distancia en altura marcadores pie } \\
\text { (puntera-talón) }(\mathrm{cm})\end{array}$} & & \\
\hline
\end{tabular}

\begin{tabular}{|l|}
\hline VELOCIDAD NORMAL \\
\hline \\
\hline
\end{tabular}

\begin{tabular}{|c|c|}
\hline CADERA & RODILLA \\
\hline Flexión & Flexión \\
\hline Extensión & Extensión \\
\hline (Rodilla $\left.0^{\circ}\right)$ & Rotación \\
\hline (Rodilla 90\%) & Valgo \\
\hline Abducción & Varo \\
\hline Adducción & TOBILLO \\
\hline Rotación int. & Flex. dorsal \\
\hline Rot. externa & Flex. plantar \\
\hline Anteversión & Supinación \\
\hline Retroversión & Pronación \\
\hline
\end{tabular}

Observaciones: 